\title{
Micropropulsion System Selection for Precision Formation Flying Satellites
}

\author{
by \\ Jeffrey Reichbach
}

B.S., Aeronautics \& Astronautics

Massachusetts Institute of Technology, 1999

SUBMITTED TO THE DEPARTMENT OF AERONAUTICS AND ASTRONAUTICS

IN PARTIAL FULFILLMENT OF THE DEGREE OF

MASTER OF SCIENCE

at the

MASSACHUSETTS INSTITUTE OF TECHNOLOGY

February 2001

(C) 2001 Massachusetts Institute of Technology

All rights reserved

Signature of Author .

Department of Aeronautics and Astronautics

January 30, 2001

Certified by ........

Doctor Raymond J. Sedwick, Thesis Supervisor

$\frown$ Department of Aeronautics and Astronautics

Certified by $\div \ldots \ldots$

Professor Manuel Martinez-Sanchez, Thesis Supervisor Department of Aeronautics and Astrona autics

Accepted by

Professor Wallace E. Vander Velde

MASSACHUSETTS INSTITUTE

OF TECHNOLOGY

SEP 112001

LIBRARIES 


\title{
Micropropulsion System Selection for Precision Formation Flying Satellites
}

\author{
by \\ Jeffrey Reichbach \\ Submitted to the Department of Aeronautics and Astronautics \\ on January 30, 2001 in Partial Fulfillment of the \\ Requirements for the Degree of Master of Science \\ at the Massachusetts Institute of Technology
}

\begin{abstract}
Several upcoming scientific interferometry missions (ST-3, LISA, TPF, MAXIM and SPECS) will push the limits of precision positioning of satellites. These spacecraft will require position and attitude control actuation on exceedingly small scales, which has not previously been performed. The several candidate propulsion systems for these missions include: colloid thrusters, field emission electrostatic propulsion thrusters (FEEP), pulsed plasma thrusters (PPT) and miniature cold gas thrusters. In order to assess the appropriateness of each of the candidate micropropulsion systems, a model of each is constructed. The models created are conglomerations of basic physical concepts and empirically founded relationships. Emphasis is placed on the determination of key operating parameters that are most relevant to design at the system level. Along with models of propulsion system performance, a common set of higher level metrics based on the GINA (Generalized Information Network Analysis) method is defined to allow the various propulsion concepts to be compared. High-level propulsion system design has been performed for each mission, employing the propulsion models created. These designs are evaluated according to the metrics developed and judgements are made as to which propulsion system is the most useful for each set of requirements.
\end{abstract}

Thesis Supervisors:

Dr. Raymond J. Sedwick

Dept. of Aeronautics and Astronautics

Prof. Manuel Martinez-Sanchez

Dept. of Aeronautics and Astronautics 


\section{ACKNOWLEDGMENTS}

The author would like to thank the Air Force Research Laboratory for their support of this work under the Grand Challenges in Space Technology: Distributed Satellite Systems Contract \#: F29601-97-K-0010. In addition, the author would like to acknowledge the fiscal officer for this contract, Sharon Leah Brown, who consistently goes out of her way to improve the lives of all the students in the lab.

Many thanks goes out to all the members of the Space Systems Laboratory for providing a rich and dynamic environment in which to work. In particular, Karen Marais and Edmund Kong spent a considerable amount of their personal time helping me to debug and refine the Matlab scripts for my thruster simulations. Their generosity is duly appreciated. And, John Enright, whom I shared an office with during my time in the SSL, was always helpful in answering even the most inane of my queries with thoughtful and useful answers, in addition to all the crossword puzzles he, Karen and I collaborated on and conquered.

So many other people have been involved in the process of creating this thesis, in subtle but important ways, that they can not all be named. However, there are several people whom it would be a travesty not to thank explicitly.

First, I would like to thank Professor David Miller for initially offering me a research assistantship in the SSL. Without his offer I would not have had the opportunity to do this work in the first place. I have learned a lot and feel that the past year and a half that I have spent as a part of the lab have had a strongly positive influence on shaping my future.

If the opportunity to do my master's degree with the SSL began with Professor Miller's offer, then my motivation to go through with the degree and complete this thesis began with a series of discussions I had with Lee Knight. She has been endlessly supportive of my endeavor. I cannot thank her enough for helping me over the mental hurdle of believing that I could actually finish my thesis.

After having the opportunity and the motivation to do this work, there was the subtle issue of actually doing the work to be dealt with. Like many research projects, this one wound its way through ideas exploring many avenues not visible in this written record. But, when dead ends and awkward transitions were encountered, I was lucky enough to have the capable and friendly guidance of my advisors: Dr. Raymond Sedwick and Professor Manuel Martinez-Sanchez.

If having two thesis advisors coming at a project from two rather different points of view (high level systems engineering and propulsion system design and analysis) might seem like an exercise for a rubber band, I wouldn't know anything about it. My pair of advisors fit my project like yin and yang; each one's strengths complementing the other's perfectly. 
I don't mean to say that their fortes are in complete opposition, rather that the roles they assumed fit together seamlessly.

Professor Martinez-Sanchez has been a role model and teacher to me for five years, since the first UROP project (electrodynamic tether thermal equilibrium modeling) I worked on during my freshman year. I never cease to be amazed at his depth, breadth and expert use of knowledge. I am greatly indebted to him for sharing a portion of his expertise and guidance with me.

I have known Ray for about four years, ever since he was the 'Unified TA' my sophomore year. It became obvious very quickly that Ray always goes well above and beyond the charge of his official responsibilities. Accordingly, he has been so much more than just an advisor to me. In addition to his amazing aptitude and willingness to 'work' any problem posed to him, he's been a great friend. He's provided so many invaluable reality checks and encouragements and constantly pushed me to excel, not to mention all the laughs we've shared.

Last, and by all means the antithesis of least, I want to thank my friends and family. The friends I have made over the past five and a half years here at MIT are the best I've had. I won't try to name them all for fear of leaving some out... You know who you are and now I hope you know how much I appreciate your support and friendship.

My family (my mother, father and sister) have been encouraging, supportive (emotionally, intellectually and financially), loving, challenging and understanding me from the very beginning. I owe them much for all they've done, been through and put up with. They and I both know that I would not be here doing what I'm doing (both figuratively and literally) without them.

This thesis is dedicated with love to the memory of my grandfathers, Harry Reichbach and Israel Leo Susman. Their confidence in my ability has helped me to achieve my grandest aspirations. I hope that I fulfill their expectations. They will forever remain a source of inspiration. 


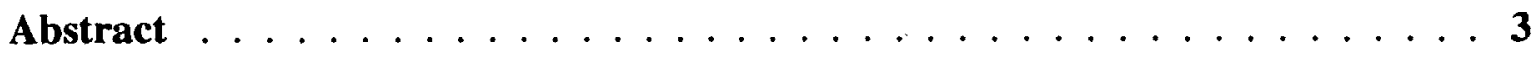

Acknowledgments $\ldots \ldots \ldots \ldots \ldots \ldots \ldots$

Nomenclature .......................... 19

Chapter 1. Introduction $\ldots \ldots \ldots \ldots \ldots \ldots \ldots$

1.1 Motivation ......................... 23

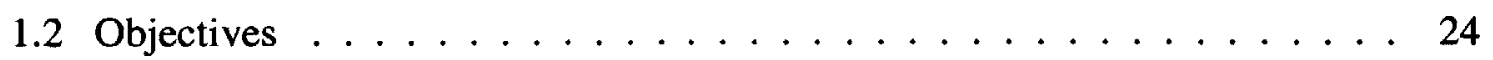

1.3 Background Work . . . . . . . . . . . . . . . . 24

1.4 Overview ........................ 25

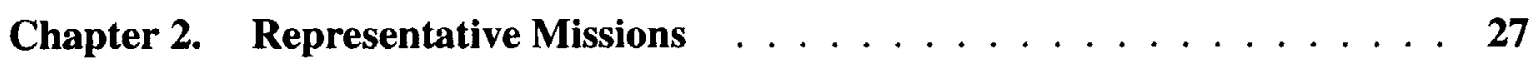

2.1 Interferometry Background $\ldots \ldots \ldots \ldots \ldots \ldots \ldots$

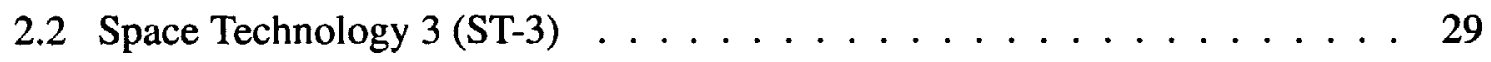

2.2 .1 Overview . . . . . . . . . . . . . . . 29

2.2 .2 Given Requirements . . . . . . . . . . . . . . 30

2.3 Terrestrial Planet Finder $(\mathrm{TPF}) \ldots \ldots \ldots \ldots \ldots \ldots$

2.3 .1 Overview ......................... 31

2.3 .2 Given Requirements . . . . . . . . . . . . . . 33

2.4 Laser Interferometry Space Antenna (LISA) . . . . . . . . . . . . . 33

2.4 .1 Overview . . . . . . . . . . . . . . 33

2.4 .2 Given Requirements . . . . . . . . . . . . . 36

2.5 Micro Arcsecond X-ray Imaging Mission (MAXIM) $\ldots \ldots \ldots \ldots$

2.5 .1 Overview . . . . . . . . . . . . . . 36

2.5 .2 Given Requirements . . . . . . . . . . . . . . . . 37

2.6 Submillimeter Probe of the Evolution of Cosmic Structure (SPECS) _ . . 39

2.6 .1 Overview . . . . . . . . . . . . . . . . 39

2.6 .2 Given Requirements . . . . . . . . . . . . . . . 41

Chapter 3. Metrics for Propulsion System Selection . . . . . . . . 43

3.1 Overview of the Generalized Information Network Analysis (GINA) Metrics 43

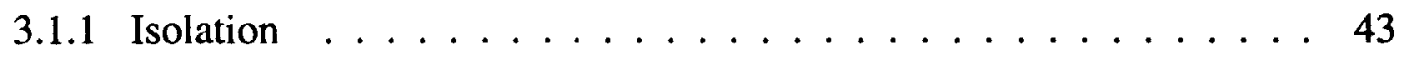

3.1 .2 Integrity $\ldots \ldots \ldots \ldots \ldots \ldots \ldots \ldots \ldots \ldots \ldots \ldots$

3.1 .3 Rate . . . . . . . . . . . . . . . 44

3.1 .4 Availability .................... 44

3.1 .5 Cost Per Function . . . . . . . . . . . . . . 44 
3.2 Interpretation and Application of GINA Metrics . . . . . . . . . . . 45

3.3 Metrics Used for Propulsion System Evaluation . . . . . . . . . . . . 46

3.3 .1 Integrity ................... 46

3.3 .2 Cost ........................ 48

Chapter 4. Propulsion Systems . . . . . . . . . . . . . . . . 53

4.1 Pulsed Plasma Thruster (PPT) . . . . . . . . . . . . . . . . . . 53

4.1.1 Background \& Fundamentals . . . . . . . . . . . . . 53

4.1.2 The PPT Dynamic Effects Model . . . . . . . . . . . . . 57

4.1.3 General Attributes and Trends . . . . . . . . . . . . . 64

4.2 Colloid Thruster . . . . . . . . . . . . . . . . . 65

4.2.1 Background \& Fundamentals . . . . . . . . . . . . . 65

4.2.2 The Colloid Thruster Control and Dynamic Effects Model . . . . . 70

4.2.3 General Attributes and Trends . . . . . . . . . . . . . . . . 78

4.3 Field Emission Electric Propulsion Thruster (FEEP) . . . . . . . . . . . 78

4.3.1 Background \& Fundamentals . . . . . . . . . . . . . . 78

4.3.2 The FEEP Thruster Dynamic Effects Model . . . . . . . . . . . 82

4.3.3 General Attributes and Trends ............... 83

4.4 Cold Gas Thruster . . . . . . . . . . . . . . . . . . . . . 85

4.4.1 Background \& Fundamentals . . . . . . . . . . . . . . 85

4.4.2 The Cold Gas Dynamic Effects Model . . . . . . . . . . . . 86

4.4 .3 General Attributes and Trends . . . . . . . . . . . . . 92

Chapter 5. Propulsion System Designs . . . . . . . . . . . . . . . . . . . . 97

5.1 Design Methodologies . . . . . . . . . . . . . . . . 97

5.1.1 PPT Methodology . . . . . . . . . . . . . . . . . 97

5.1 .2 Colloid Thruster Methodology . . . . . . . . . . . . . . . 98

5.1 .3 FEEP Thruster Methodology . . . . . . . . . . . . . . . . . 99

5.1 .4 Cold Gas Thruster Methodology . . . . . . . . . . . . . . . 100

5.2 Space Technology $3 \ldots \ldots \ldots 1 . \ldots \ldots 1$

5.2 .1 PPT Design . . . . . . . . . . . . . . . . . . 102

5.2 .2 Colloid Thruster Design . . . . . . . . . . . . . . . 104

5.2 .3 FEEP Thruster Design . . . . . . . . . . . . . . . . . . . . . . . . . . . . . . . . . . . .

5.2.4 Cold Gas Thruster Design . . . . . . . . . . . . . . . 107

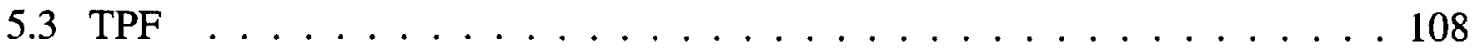

5.3 .1 PPT Design . . . . . . . . . . . . . . . . . . 109

5.3 .2 Colloid Thruster Design . . . . . . . . . . . . . 110

5.3 .3 FEEP Thruster Design . . . . . . . . . . . . . . 110 
5.3.4 Cold Gas Thruster Design . . . . . . . . . . . . . . . 111

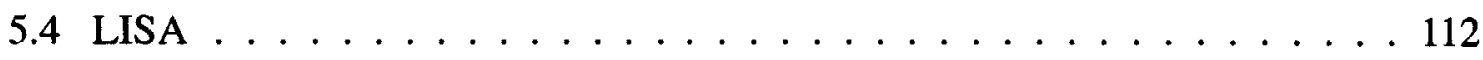

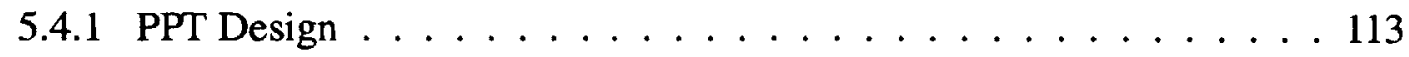

5.4 .2 Colloid Thruster Design . . . . . . . . . . . . . . . . . . . . . . . . . . . . . . .

5.4 .3 FEEP Thruster Design . . . . . . . . . . . . . . . 114

5.4 .4 Cold Gas Thruster Design . . . . . . . . . . . . . . 115

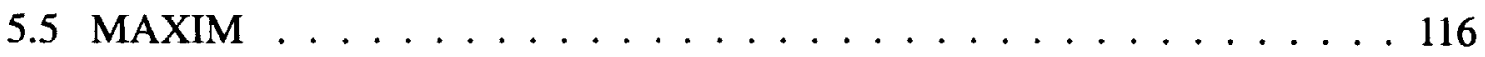

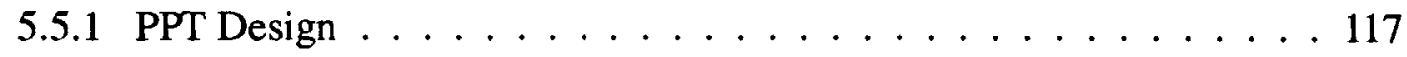

5.5 .2 Colloid Thruster Design . . . . . . . . . . . . . 117

5.5 .3 FEEP Thruster Design .................. . . 118

5.5.4 Cold Gas Thruster Design . . . . . . . . . . . . . . . 119

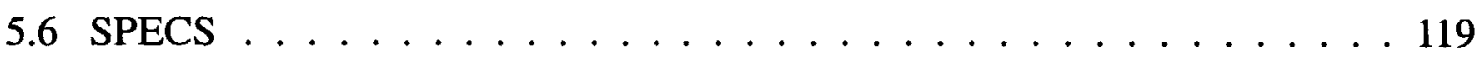

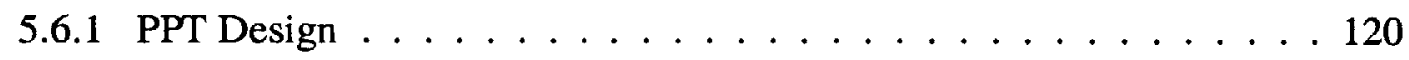

5.6 .2 Colloid Thruster Design . . . . . . . . . . . . . . 121

5.6 .3 FEEP Thruster Design . . . . . . . . . . . . . . . 122

5.6 .4 Cold Gas Thruster Design . . . . . . . . . . . . . . 123

Chapter 6. Propulsion system selection Results . . . . . . . . . . . 125

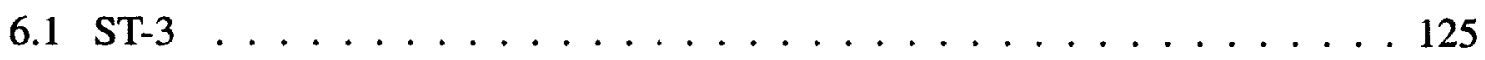

6.1 Propulsion System Selection . . . . . . . . . . . 125

6.1.2 Discussion of Trades .................... 128

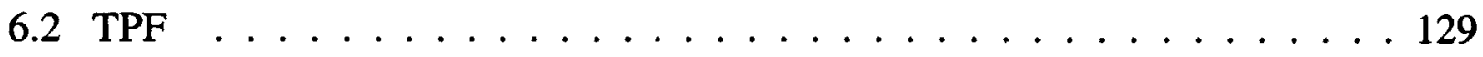

6.2.1 Propulsion System Selection . . . . . . . . . . . . . . 129

6.2 .2 Discussion of Trades . . . . . . . . . . . . . . 131

6.3 LISA . . . . . . . . . . . . . . . . . . . 132

6.3 .1 Propulsion System Selection . . . . . . . . . . . . . . 132

6.3.2 Discussion of Trades . . . . . . . . . . . . . . 135

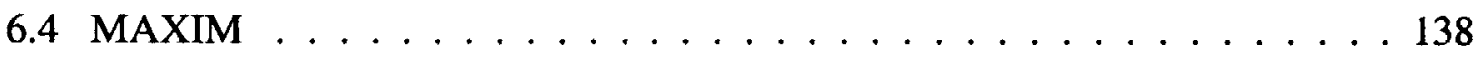

6.4.1 Propulsion System Selection . . . . . . . . . . . . . 138

6.4 .2 Discussion of Trades . . . . . . . . . . . . . . . 140

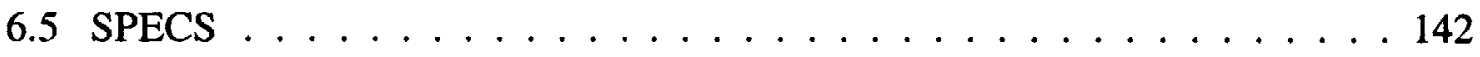

6.5 .1 Propulsion System Selection . . . . . . . . . . . . . 142

6.5.2 Discussion of Trades . . . . . . . . . . . . . . 143

6.6 Summary of Selection Trends . . . . . . . . . . . . . . 144

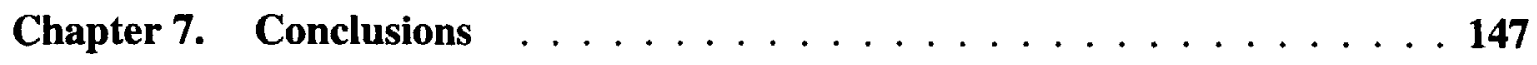

7.1 Design Recommendations . . . . . . . . . . . . . . . . 147 
7.1.1 Propulsion System Selection . . . . . . . . . . . . . . . . . 147

7.1.2 Future Propulsion System Development . . . . . . . . . . . . . . . 148

7.2 Limitations . . . . . . . . . . . . . . . . . . . . . . . . . . . 149

7.3 Future Work . . . . . . . . . . . . . . . . . . 150

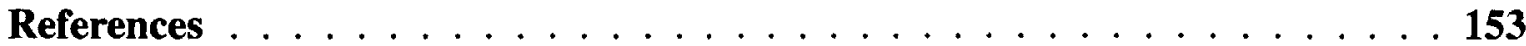

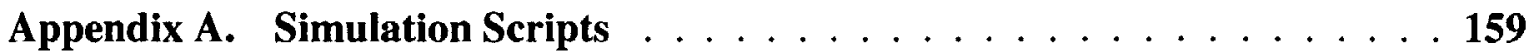

A.1 PPT Simulation Script Example . . . . . . . . . . . . . . . . . . . 159

A.2 Colloid Simulation Script Example ～. . . . . . . . . . . . . . 161

A.3 FEEP Simulation Script Example . . . . . . . . . . . . . . . . . . . . 164

A.4 Cold Gas Simulation Script Example . . . . . . . . . . . . . 165

Appendix B. Simulation REsults $\ldots \ldots \ldots \ldots$

B.1 ST-3 Simulation Results . . . . . . . . . . . . . . . . . . . . . . . . . . 169

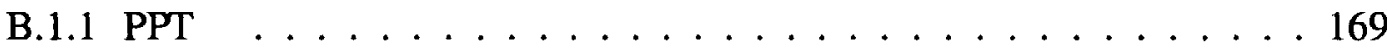

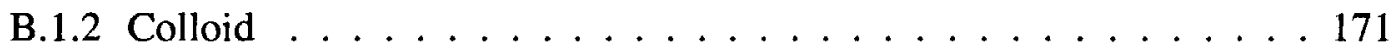

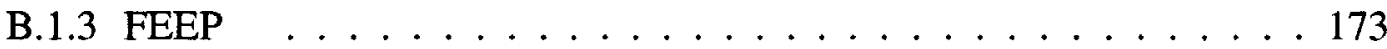

B.1.4 Cold Gas . . . . . . . . . . . . . . . . . . . . . . . . 174

B.2 TPF Simulation Results . . . . . . . . . . . . . . . . . . . . . 177

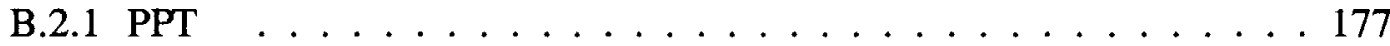

B.2.2 Colloid . . . . . . . . . . . . . . . . . . 179

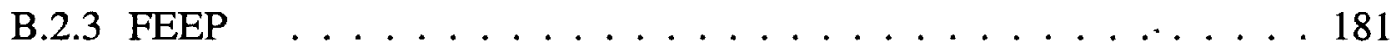

B.2.4 Cold Gas . . . . . . . . . . . . . . . . . . 182

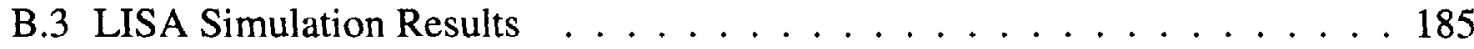

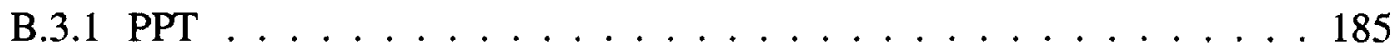

B.3.2 Colloid . . . . . . . . . . . . . . . . 186

B.3.3 FEEP . . . . . . . . . . . . . . . . . . . . 187

B.3.4 Cold Gas . . . . . . . . . . . . . . . . . . . . . . 187

B.4 MAXIM Simulation Results _. . . . . . . . . . . . . . . . . . . . 189

B.4.1 PPT . . . . . . . . . . . . . . . . . . . 189

B.4.2 Colloid . . . . . . . . . . . . . . . . 191

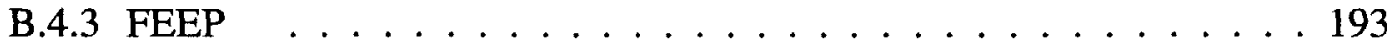

B.4.4 Cold Gas . . . . . . . . . . . . . . . . . . . . . . . . 194

B.5 SPECS Simulation Results _ . . . . . . . . . . . . . . . . 197

B.5.1 PPT . . . . . . . . . . . . . . . . . . . . 197

B.5.2 Colloid . . . . . . . . . . . . . . . . . . 198 
B.5.3 FEEP . . . . . . . . . . . . . . . . . . . 199

B.5.4 Cold Gas . . . . . . . . . . . . . . . . . 199

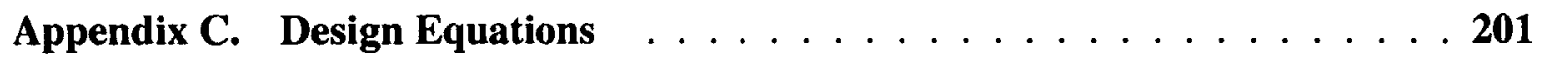

C.1 PPT Design Equations . . . . . . . . . . . . . . . . 201

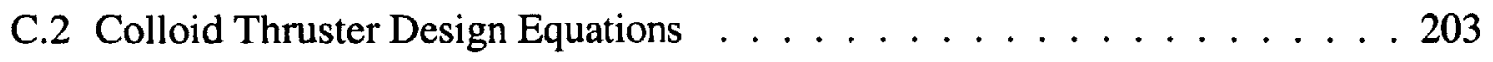

C.3 FEEP Thruster Design Equations . . . . . . . . . . . . . . . . . . 207

C.4 Cold Gas Thruster Design Equations . . . . . . . . . . . . . . . . 209 
Figure 2.1 Michelson interferometer schematic drawing showing collectors, combiner and delay line. Adapted from [Jilla,1998] \& [Kong, 1998]. . . . . . . 28

Figure 2.2 Schematic of ST-3 interferometry phase showing the collector spacecraft on the surface of a paraboloid whose focus is fixed at the combiner spacecraft [Blackwood, 1999]. . . . . . . . . . . . . . . . . 31

Figure 2.3 Artists rendition of four TPF spacecraft flying in formation, observing a distant solar system $($ white $=$ starlight, red $=$ metrology). [Beichman, 1997-

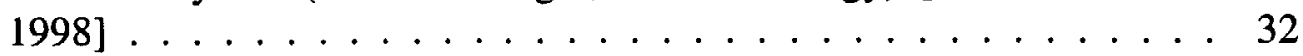

Figure 2.4 Illustration of LISA formation movement during an orbit. The green line represents the orbit of the earth (in the plane of the ecliptic) and the red line is the orbit of one LISA spacecraft (orange dot) [Bender, 1998]. . . . . 34

Figure 2.5 LISA spacecraft payload diagram. Gold squares are proof masses, shades of red represent laser path $[$ Folkner, 1998]. . . . . . . . . . . . 35

Figure 2.6 Illustration of fully deployed MAXIM formation including 33 collectors, a combiner, a delay line and a detector spacecraft [Cash, 2000]. . . . . 38

Figure 2.7 Baseline requirements for the MAXIM micro-arcsecond X-ray interferometer shown in the cells shaded green [Cash (Web Site), 2000]. . . . . . 39

Figure 2.8 Post-big bang photon intensity plotted on a log scale showing the significance of surveying the universe in the submillimeter wavelengths [Leisawitz, 2000]. . . . . . . . . . . . . . . . . 40

Figure 2.9 Tethered three mirror design schematic of SPECS spacecraft during imaging from the target's perspective [Leisawitz, 2000]. . . . . . . . . 41

Figure 4.1 Breech-fed pulsed plasma thruster (PPT) schematic . . . . . . . 54

Figure 4.2 Idealized dead-band scenario schematic under a constant disturbance force [London, 1996] (adapted). . . . . . . . . . . . . . . 58

Figure 4.3 PPT cumulative impulse plot showing disturbance in red and thruster actua-

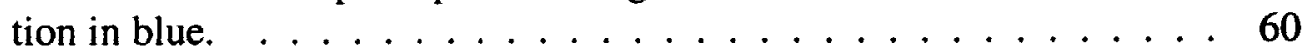

Figure 4.4 Disturbance force used for the example simulation runs. . . . . . . 61

Figure 4.5 PPT thrusting profile for position maintenance, exemplified. . . . . 62

Figure 4.6 Net acceleration power spectral density (PSD) example. . . . . . . . 62

Figure 4.7 Position power spectral density example (from trajectory). . . . . 63

Figure 4.8 One dimensional spacecraft trajectory about a desired position. . . . . 64

Figure 4.9 Single-needle colloid thruster schematic. . . . . . . . . . . 66

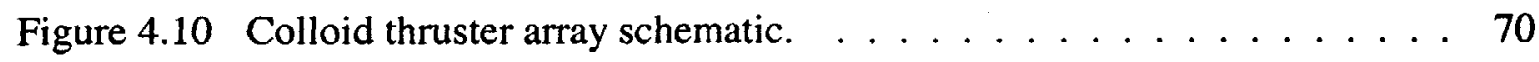

Figure 4.11 Illustration of change in fluid meniscus at needle tip necessary before colloid emission can begin. . . . . . . . . . . . . 72 
Figure 4.12 Colloid thruster thrust profile plotted with . . . . . . . . . . 75

Figure 4.13 Colloid thruster array accelerator voltage for the example simulation. . 76

Figure 4.14 Spacecraft trajectory with colloid thruster maintaining position. . . . . 76

Figure 4.15 Net acceleration PSD for spacecraft with colloid thruster firing. . . . 77

Figure 4.16 Position PSD for spacecraft with colloid thruster firing. . . . . . . . 77

Figure 4.17 FEEP thruster schematic illustration (not to scale). . . . . . . . . . . 79

Figure 4.18 Schematic of indium FEEP [Steiger, 2000] (adapted). . . . . . . . . . 80

Figure 4.19 FEEP thruster thrust profile example. . . . . . . . . . . . . . . 83

Figure 4.20 FEEP thruster trajectory example. . . . . . . . . . . . . . . . . . 84

Figure 4.21 FEEP thruster thrust PSD [Steiger, 2000]. . . . . . . . . . . . . . . . 84

Figure 4.22 Cold gas thruster schematic diagram. . . . . . . . . . . . . . 85

Figure 4.23 Thrust profile for cold gas thruster position maintenance example. . . 88

Figure 4.24 Cold gas thruster trajectory example. . . . . . . . . . . . . . . . . . 89

Figure 4.25 Cold gas thruster position PSD example. . . . . . . . . . . . . . . . . 89

Figure 4.26 Cold gas thruster acceleration PSD example. . . . . . . . . . . . . 90

Figure 4.27 Schematic drawing of solenoid and plunger used in scaling analysis . . 93

Figure 5.1 PPT design methodology flow diagram. . . . . . . . . . . . . . 98

Figure 5.2 Colloid thruster design methodology flow diagram. . . . . . . . . . . 99

Figure 5.3 FEEP thruster design methodology diagram. . . . . . . . . . . 100

Figure 5.4 Cold gas thruster design methodology diagram. . . . . . . . . . . . 101

Figure 6.1 ST-3 propulsion system total costs including development, hardware and launch. . . . . . . . . . . . . . . . 128

Figure 6.2 TPF propulsion system total costs including development, hardware and launch. . . . . . . . . . . . . . . 131

Figure 6.3 LISA (2 years) propulsion system total costs including development, hardware and launch. . . . . . . . . . . . . . . . . . 135

Figure 6.4 LISA (10 years) propulsion system total costs including development, hardware and launch. . . . . . . . . . . . . . . . 136

Figure 6.5 Power spectral density of position (cold gas thruster) after high pass filtering with requirement from LISA indicated by the red line . . . . . . . 136

Figure 6.6 Power spectral density of position (colloid thruster) after high pass filtering with requirement from LISA indicated by the red line . . . . . . 137

Figure 6.7 MAXIM (collector spacecraft) propulsion system total costs including development, hardware and launch. . . . . . . . . . . . . . 140 
Figure 6.8 MAXIM (detector spacecraft) propulsion system total costs including development, hardware and launch. . . . . . . . . . . . . . . . 141

Figure 6.9 SPECS propulsion system total costs including development, hardware and launch. . . . . . . . . . . . . . . . . . . 143

Figure 6.10 Three dimensional design (dead-band, Dv, spacecraft mass) space containing the five missions. . . . . . . . . . . . . . . . . . 144

Figure B.1 Simulation results for the ST-3 collector spacecraft using PPTs. . . . 169

Figure B.2 Simulation results for the ST-3 combiner spacecraft using PPTs. . . . 170

Figure B.3 Simulation results for the ST-3 collector spacecraft using colloid thrusters. 171

Figure B.4 Simulation results for the ST-3 combiner spacecraft using colloid thrusters. 172

Figure B.5 Simulation results for the ST-3 collector spacecraft using a FEEP system. . 173

Figure B.6 Simulation results for the ST-3 combiner spacecraft using a FEEP system. 174

Figure B.7 Simulation results for the ST-3 collector spacecraft using cold gas thrusters. 174

Figure B.8 Simulation results for the ST-3 combiner spacecraft using cold gas thrusters. 175

Figure B.9 Simulation results for the TPF collector spacecraft using PPTs. . . . 177

Figure B.10 Simulation results for the TPF combiner spacecraft using PPTs. . . . 178

Figure B.11 Simulation results for the TPF collector spacecraft using colloid thrusters. 179

Figure B.12 Simulation results for the TPF combiner spacecraft using colloid thrusters. 180

Figure B.13 Simulation results for the TPF collector spacecraft using a FEEP system. . 181

Figure B.14 Simulation results for the TPF combiner spacecraft using a FEEP system. 181

Figure B.15 Simulation results for the TPF collector spacecraft using cold gas thrusters. 182

Figure B.16 Simulation results for the TPF combiner spacecraft using cold gas thrusters. 183

Figure B.17 Simulation results for the LISA spacecraft using PPTs. . . . . . . . 185

Figure B.18 Simulation results for the LISA spacecraft using colloid thrusters. . . 186 
Figure B.19 Simulation results for the LISA spacecraft using a FEEP system. . . . 187

Figure B.20 Simulation results for the LISA spacecraft using cold gas thrusters. . . 187

Figure B.21 Simulation results for the MAXIM collector spacecraft using PPTs. . . 189

Figure B.22 Simulation results for the MAXIM detector spacecraft using PPTs. . . 190

Figure B.23 Simulation results for the MAXIM collector spacecraft using colloid thrust-

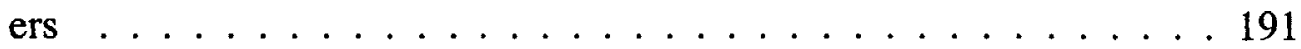

Figure B.24 Simulation results for the MAXIM detector spacecraft using colloid thrust-

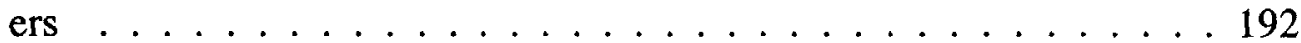

Figure B.25 Simulation results for the MAXIM collector spacecraft using a FEEP system. . . . . . . . . . . . . . . . . 193

Figure B.26 Simulation results for the MAXIM detector spacecraft using a FEEP system. 193

Figure B.27 Simulation results for the MAXIM collector spacecraft using cold gas thrusters. . . . . . . . . . . . . . . . . . . . . . . 194

Figure B.28 Simulation results for the MAXIM detector spacecraft using cold gas thrusters. . . . . . . . . . . . . . . . . . . 195

Figure B.29 Simulation results for the SPECS spacecraft using PPTs. . . . . . . . . 197

Figure B.30 Simulation results for the SPECS spacecraft using colloid thrusters. . . 198

Figure B.31 Simulation results for the SPECS spacecraft using a FEEP system. . 199

Figure B.32 Simulation results for the SPECS spacecraft using cold gas thrusters. . 199 
TABLE 2.1 TPF example concept design overview [Beichman, 1997-1998]. . . . 33

TABLE 2.2 SPECS design parameters [Leisawitz (Web site), 2000] . . . . . . 41

TABLE 3.1 NASA Technology Readiness Levels (TRLs) [Mankins, 1995] . . . . 51

TABLE 4.1 Performance data from flight and laboratory PPT testing [Burton, 1998]. . 54

TABLE 4.2 Summary of example simulation parameters $\ldots \ldots \ldots$

TABLE 4.3 Block and needle increments for the SPECS colloid thruster design. . 72

TABLE 4.4 FEEP life test data, highlighting steady-state thrust variation [Marcuccio, 2000]. . . . . . . . . . . . . . . . . 82

TABLE 4.5 Moog cold gas microthruster specification [Bzibziak, 2000]. . . . . 87

TABLE 4.6 Simulation parameters for cold gas thruster example analysis. . . . 87

TABLE 4.7 Cold gas thruster model variables and units for dimensional analysis . 90

TABLE 5.1 ST3 Pulsed Plasma Thruster Design Specifications $\ldots \ldots$. . . . . 103

TABLE 5.2 ST3 Colloid Thruster Design Specifications . . . . . . . . . . 105

TABLE 5.3 ST3 FEEP System Design Specifications $\ldots \ldots \ldots$. . . . . . . 106

TABLE 5.4 ST3 Cold Gas Thruster Design Specifications . . . . . . . . . . 108

TABLE 5.5 TPF Pulsed Plasma Thruster Design Specifications $\ldots \ldots$. . . . . 109

TABLE 5.6 TPF Colloid Thruster Design Specifications $\ldots \ldots \ldots \ldots$

TABLE 5.7 TPF FEEP System Design Specifications $\ldots \ldots \ldots \ldots$

TABLE 5.8 TPF Cold Gas Thruster Design Specifications . . . . . . . . . 111

TABLE 5.9 LISA Pulsed Plasma Thruster Design Specifications (Note: mass figures in parentheses denote 10 year mission lifetime, those without are for the nominal 2 year mission $\ldots \ldots \ldots \ldots \ldots \ldots \ldots \ldots \ldots$

TABLE 5.10 LISA Colloid Thruster Design Specifications (Note: mass figures in parentheses denote 10 year mission lifetime, those without are for the nominal 2 year mission) . . . . . . . . . . . . . 113

TABLE 5.11 LISA FEEP System Design Specifications (Note: mass figures in parentheses denote 10 year mission lifetime, those without are for the nominal 2 year mission) . . . . . . . . . . . . . . . . 114

TABLE 5.12 LISA Cold Gas Thruster Design Specifications (Note: mass figures in parentheses denote 10 year mission lifetime, those without are for the nominal 2 year mission) . . . . . . . . . . . . . 115

TABLE 5.13 MAXIM Pulsed Plasma Thruster Design Specifications . . . . . . 117

TABLE 5.14 MAXIM Colloid Thruster Design Specifications $\ldots \ldots \ldots$ 
TABLE 5.15 MAXIM FEEP System Design Specifications . . . . . . . . . . 118

TABLE 5.16 MAXIM Cold Gas Thruster Design Specifications ～. . . . . . . 119

TABLE 5.17 SPECS Pulsed Plasma Thruster Design Specifications ～. . . . . . 120

TABLE 5.18 SPECS Colloid Thruster Design Specifications ～. . . . . . . . . 121

TABLE 5.19 SPECS FEEP System Design Specifications . . . . . . . . . . 122

TABLE 5.20 SPECS Cold Gas Thruster Design Specifications $\ldots \ldots . . \ldots 123$

TABLE 6.1 ST-3 Propulsion System Table . . . . . . . . . . . . . . . . 127

TABLE 6.2 TPF Propulsion System Selection Table . . . . . . . . . . . . . . 130

TABLE 6.3 LISA Propulsion System Selection Table . . . . . . . . . . . 133

TABLE 6.4 MAXIM Propulsion System Selection Table . . . . . . . . . . . . 139

TABLE 6.5 SPECS Propulsion System Selection Table . . . . . . . . . . . 142

TABLE 6.6 Propulsion System Cost-Based Selection Summary $\ldots \ldots$. . . . . . 145 


\section{NOMENCLATURE}

\begin{tabular}{|c|c|}
\hline$A$ & $\operatorname{area}\left[\mathrm{m}^{2}\right]$ \\
\hline$b$ & baseline $[\mathrm{m}]$ \\
\hline$B$ & magnetic flux $\left[\mathrm{Wb} / \mathrm{m}^{2}\right]$ \\
\hline$c$ & specific heat capacity $\left[\mathrm{J} / \mathrm{kg} /{ }^{\circ} \mathrm{K}\right]$ \\
\hline$C_{F}$ & thrust coefficient [ ] \\
\hline Cost $_{i}$ & dollar value of or due to i $[\$]$ \\
\hline$D O D$ & depth of discharge [ ] \\
\hline$d$ & diameter $[\mathrm{m}]$ \\
\hline$d L$ & length increment $[\mathrm{m}]$ \\
\hline$e$ & electron charge $[\mathrm{C}]$ \\
\hline$E$ & electric field strength $[\mathrm{V} / \mathrm{m}]$ \\
\hline$E_{o}$ & capacitor energy $[\mathrm{J}]$ \\
\hline$f$ & frequency $[\mathrm{Hz}]$ \\
\hline$f_{s c}$ & scale factor [] \\
\hline$F$ & thrust $[\mathrm{N}]$ \\
\hline FOS & factor of safety [ ] \\
\hline$g$ & gravitational acceleration $\left[\mathrm{m} / \mathrm{s}^{2}\right]$ \\
\hline$h$ & fuel bar height [m] \\
\hline$I_{a}$ & accelerator current $[\mathrm{A}]$ \\
\hline$I_{e}$ & emitter current $[\mathrm{A}]$ \\
\hline$I_{b i t}$ & minimum impulse bit $[\mathrm{Ns}]$ \\
\hline$I_{\max }$ & $\operatorname{maximum}$ current $[\mathrm{A}]$ \\
\hline$I_{N}$ & current in single colloid needle [A] \\
\hline$I_{s p}$ & specific impulse $[\mathrm{s}]$ \\
\hline$I_{t}$ & total impulse [Ns] \\
\hline$K$ & thermal conductivity $\left[\mathrm{W} / \mathrm{m} /{ }^{\circ} \mathrm{K}\right]$ \\
\hline$k$ & Boltzmann's constant $\left[\mathrm{J} /{ }^{\circ} \mathrm{K}\right]$ \\
\hline$l$ & length [m] \\
\hline$L$ & inductance $[\mathrm{H}]$ \\
\hline$m_{i}$ & mass of component $i[\mathrm{~kg}]$ \\
\hline$\dot{m}$ & mass flow rate $[\mathrm{kg} / \mathrm{s}]$ \\
\hline$M$ & Mach Number \\
\hline$N$ & integer [ ] \\
\hline$p_{i}$ & pressure in device $i$ or at point $i[\mathrm{~Pa}]$ \\
\hline$P_{i}$ & power in device $i$ or at point $\mathrm{i}$ [W] \\
\hline$P R F$ & pulse repetition frequency $\left[\mathrm{s}^{-1}\right]$ \\
\hline$P S D$ & power spectral density [units $/ \mathrm{Hz}$ ] \\
\hline$q$ & charge $[\mathrm{C}]$ \\
\hline$Q$ & volumetric flow rate $\left[\mathrm{m}^{3} / \mathrm{s}\right]$ \\
\hline & radius $[\mathrm{m}]$ \\
\hline
\end{tabular}




\begin{tabular}{|c|c|}
\hline$R$ & gas constant $\left[\mathrm{J} / \mathrm{kg} /{ }^{\circ} \mathrm{K}\right]$ \\
\hline$S$ & learning curve slope [ ] \\
\hline$t$ & time $[s]$ \\
\hline$T_{i}$ & temperature at point $\mathrm{i}\left[{ }^{\circ} \mathrm{K}\right]$ \\
\hline$T R L$ & technology readiness level [] \\
\hline$u$ & $\mathrm{u}-\mathrm{v}$ (spatial fourier) plane point [ ] \\
\hline$v$ & $\mathrm{u}-\mathrm{v}$ (spatial fourier) plane point [ ] \\
\hline $\mathrm{v}_{i}$ & velocity at point $\mathrm{i}[\mathrm{m} / \mathrm{s}]$ \\
\hline$V$ & volume $\left[\mathrm{m}^{3}\right]$ \\
\hline $\mathrm{V}_{i}$ & voltage of component $i[\mathrm{~V}]$ \\
\hline$w$ & fuel bar width $[\mathrm{m}]$ \\
\hline$w_{p}$ & pulse width [s] \\
\hline $\mathscr{W}_{m}$ & magnetic work [Nm] \\
\hline$x_{i}$ & spatial coordinate at point $i[\mathrm{~m}]$ \\
\hline$y_{i}$ & spatial coordinate at point $\mathrm{i}[\mathrm{m}]$ \\
\hline$\alpha$ & plume divergence angle (vertical) [radians] \\
\hline$\beta$ & plume divergence angle (horizontal) [radians] \\
\hline$\beta_{i}$ & ratio of ion current to total current [ ] \\
\hline$\varepsilon_{d}$ & dielectric constant [ ] \\
\hline$\varepsilon_{o}$ & permittivity of free space $[\mathrm{F} / \mathrm{m}]$ \\
\hline$\Delta p$ & path length difference $[\mathrm{m}]$ \\
\hline$\Delta \mathrm{v}$ & velocity increment $[\mathrm{m} / \mathrm{s}]$ \\
\hline$\Delta \mathrm{x}$ & dead-band width $[\mathrm{m}]$ \\
\hline$\gamma$ & specific heat ratio [ ] \\
\hline$\gamma_{\text {st }}$ & coefficient of surface tension $[\mathrm{Nm}]$ \\
\hline$\eta$ & non-dimensional flow rate [ ] \\
\hline$\eta_{p}$ & power efficiency [ ] \\
\hline$\eta_{t}$ & thrust efficiency [ ] \\
\hline$\theta$ & angle [radians] \\
\hline$\kappa$ & electrical conductivity $[\mathrm{Si} / \mathrm{m}]$ \\
\hline$\lambda$ & wavelength [m] \\
\hline$\mu$ & viscosity $[\mathrm{Pa} \mathrm{s}]$ \\
\hline$\mu_{o}$ & permeability of free space $\left[\mathrm{N} / \mathrm{A}^{2}\right]$ \\
\hline$\xi$ & fraction of ideal dead-band cycle spent thrusting [ ] \\
\hline$\rho$ & density $\left[\mathrm{kg} / \mathrm{m}^{3}\right]$ \\
\hline$\sigma$ & plasma conductivity $[\mathrm{Si} / \mathrm{m}]$ \\
\hline$\sigma_{y}$ & yield stress $[\mathrm{Pa}]$ \\
\hline$\phi$ & ratio of charge to mass ratios []$^{\mathrm{a}}$ \\
\hline$\omega$ & frequency $[\mathrm{rad} / \mathrm{s}]$ \\
\hline$(-)_{1}$ & chamber \\
\hline$(-)_{2}$ & exit \\
\hline$(-)_{3}$ & ambient \\
\hline$(-)_{c a p}$ & capacitor \\
\hline$(-)_{d i c}$ & discharge initiation circuitry \\
\hline$(-)_{\text {feed }}$ & propellant feed system \\
\hline
\end{tabular}




$\begin{array}{ll}(-)_{\text {neutral }} & \text { neutralizer } \\ (-)_{\text {pu }} & \text { power processing unit } \\ (-)_{\text {prop }} & \text { propellant } \\ (-)_{s} & \text { structure } \\ (-)_{\text {shot }} & \left.\text { single PPT pulse (having impulse }=I_{b i t}\right) \\ (-)_{t} & \text { throat } \\ (-)_{\text {tank }} & \text { tank } \\ (-)_{\text {valve }} & \text { valve }\end{array}$

a. No, this is not a typographical error. $\phi$ is the ratio the charge to mass ratio of the droplets to the charge to mass ratio of the ions in a colloid thruster operating in the regime where both ions and droplets are being emitted. See Section 4.2.1 for further information. 


\section{Chapter 1}

\section{INTRODUCTION}

\subsection{Motivation}

Several of NASA's upcoming scientific missions are pushing the limits of precision positioning of satellites, with respect to each other and their orbits. Near-term missions of this nature include ST-3, an optical space interferometry and formation flight demonstration mission, ST-5, a precision formation flying demonstrator for a follow-on gravity wave detection mission, LISA. These three missions, along with several other future mission, will require position and attitude control actuation on exceedingly small scales, for which propulsion systems with smaller thrusts and greater precision than those available today are being designed. Along with a need for better models of propulsion system performance, a common set of metrics must be defined to allow widely varying concepts to be compared fairly, which will allow for the extraction of the maximum utility from these missions.

The decision of which propulsion system to use for each mission is an important one with both immediate and long term effects. Not only are mass, power and maneuvering requirements important for the missions currently being designed, but the propulsion technologies developed for the near term missions will greatly influence their use in future missions with similar needs, as a result of heritage effects on the system cost. 


\subsection{Objectives}

Pushing the limits of space science requires technological advances in individual subsystems and components in addition to methods used to integrate them to form an efficient total system. This thesis endeavors to investigate the capabilities of various state-of-theart space propulsion systems, as well as to determine their effects on large scale space system performance, and lay a foundation for methods of analysis that increase system utility.

One of the most intriguing and currently popular areas of space science involves imaging at high resolutions. Interferometry has become the premier tool for creating high resolution images, at various wavelengths, without expending as many resources as traditional imaging methods. Currently, interferometric techniques are baselined for space missions involving optical, infra-red, $x$-ray, sub-millimeter and gravitational wave 'imaging'. Interferometry payloads put many special and more restrictive demands on the space systems that support them. One of the most impacting of these demands is the need for position and attitude maintenance that far exceed conventional limits.

These demands are constantly requiring space system hardware and design techniques to be rethought. This thesis attempts to expand the traditional evaluation of propulsion system performance, and in doing so allow for more ambitious space missions and more efficient means of designing them.

Additionally, the characterization of the propulsion systems presented herein is hoped to give a suggestion for areas in which to emphasize future propulsion technology development, thus providing the ability to do more with space missions sooner and with less expense.

\subsection{Background Work}

The work described in this thesis is primarily based on two bodies of prior work, electric propulsion and systems engineering research. The systems engineering aspect of this 
study is based mainly on work done by Graeme Shaw for his doctoral thesis [Shaw, 1998] at the MIT Space Systems Laboratory and the well known compilation, Space Mission Analysis and Design [Larson, 1992]. Work on more general propulsion system trade studies was performed by London in his master's thesis [London, 1996]. The propulsion research comes from a number of sources both recent and past. It includes pulsed plasma thruster research primarily by William Guman in the 1960s [Guman, 1968] and 1970s [Guman, 1975] and Vondra and Thomassen in the 1970s [Vondra,1974], more recent (1990s) work by Robert Burton [Burton, 1998], Peter Turchi [Turchi, 1999] and Yiangos Mikelllides [Mikellides, 1999] and very recently by Frank Gulczinski and Gregory Spanjers [Gulczinski, 2000]. Field emission electric propulsion research has primarily been performed at Centrospazio in Italy by Salvo Marcuccio [Marcuccio, 1998], [Marcuccio, 2000], Angelo Genovese and Mariano Andrenucci [Andrenucci, 1993] and at the Austrian Research Center Seibersdorf by Steiger, Genevose [Steiger, 2000] and Tajmar [Tajmar, 2000]. Fundamental research that helped spur the colloid thruster concept was done over eighty years ago by Zeleny [Zeleny, 1917]. Colloid thruster research proper has been carried out by numerous people both in the 1960s [Taylor, 1964] and 1970s [Jackson, 1972] as well as more recently starting in the 1990s. Recent major contributions have been made by Juan Fernandez de la Mora [Fernandez de la Mora, 1994] and colleagues, which include Manuel Gamero [Gamero, 2000], Vlad Hruby [Hruby, 2000] and Manuel Martinez-Sanchez [Martinez-Sanchez, 1999a], [Martinez-Sanchez, 1999b]. The majority of the published work regarding micro-cold gas thrusters has been done commercially by various researchers at MOOG, Inc., Space Products Division [Bzibziak, 2000].

\subsection{Overview}

The layout of this thesis generally follows the methodology used to perform large scale system design. The fundamental goals are set first and the specific systems by which these goals are to be achieved are described in detail (Chapter 2). Then, a definition of what meeting the goals that have been set means quantitatively is defined (Chapter 3 ). Next, the means and methods used to meet those goals are modeled (Chapter 4). Prelimi- 
nary work is then carried out to define the designs that are to be evaluated (Chapter 5). Finally, a trade study is performed to determine the range of possible designs and arrive at a best solution (Chapter 6 ).

Specifically, five interferometric space science missions are described and their requirements, which need to be flowed down to constrain the propulsion system designs, are discussed in Chapter 2. Then, a description and justification for the metrics used in evaluating the propulsion systems design is given in Chapter 3. The following chapter, Chapter 4, discusses each propulsion system's fundamentals and presents the models used for analysis. Next, a compilation of preliminary designs for each propulsion system-mission combination is created in Chapter 5. Lastly, the propulsion system designs are evaluated according to the aforementioned metrics in Chapter 6, resulting in a best propulsion system for each mission and general trends discovered throughout the trade space. 


\section{Chapter2}

\section{REPRESENTATIVE MISSIONS}

\subsection{Interferometry Background}

The interferometer was invented by Albert A. Michelson in about 1880. Michelson originally designed the interferometer for ether-drift experiments to prove the existence of the medium, which was thought to explain the propagation of light. Interferometers are now widely used for spectroscopy, the study of thin films, the testing of precision optics, measurements of refractive indices, and both radio and optical astronomy.

The advantage of interferometry is that it can produce images of stars with a higher angular resolution than is possible with conventional telescopes. An interferometer is composed of an array of several separate telescopes, which redirect starlight to a central location where interference fringes are formed. The available angular resolution depends only on the telescope separations, which can be made much larger than the diameter of conventional telescopes, whose resolution is restricted by their aperture diameter.

For example, an optical interferometer samples the wavefronts of light emitted by a source at two or more separate locations and recombines the sampled wavefronts to produce interference fringes. The wavefronts add constructively or destructively, depending on the path difference between the wavefronts, and produce fringes that appear as bright and dark bands. If the pathlength in one arm of the interferometer is changed by even a frac- 
tion of a wavelength, the fringes will appear to move. This extreme sensitivity to minute path variations makes interferometry a powerful tool [Lawson,2000].

The output of the interfered light waves is a complex valued signal containing the magnitude and phase of the image's spatial Fourier component, which depends on the particular aperture separation projected onto the line of sight (the line perpendicular to the wavefront). In order to accomplish the proper interference of the different light beams, optical delay lines are used to adjust the phase of the signal to make up for the difference in the original optical pathlength [Kong,1998]. A schematic of a simple Michelson interferometer is shown in Figure 2.1. The optical delay line (ODL) on the collector nearer the source

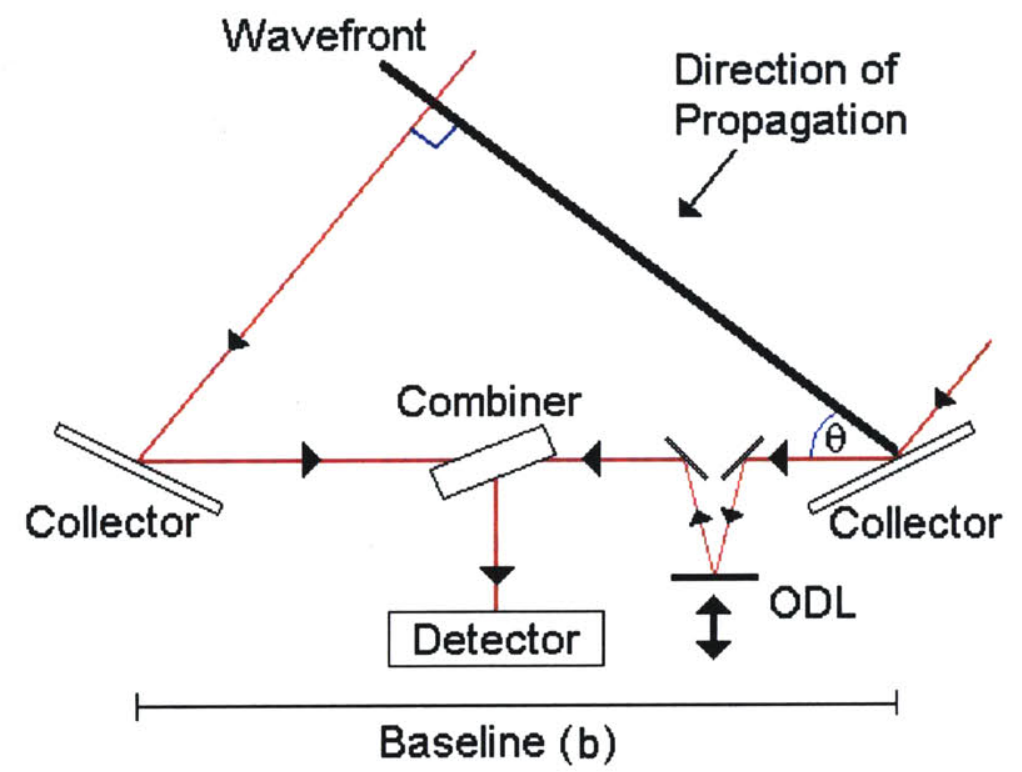

Figure 2.1 Michelson interferometer schematic drawing showing collectors, combiner and delay line. Adapted from [Jilla,1998] \& [Kong,1998].

must make up a pathlength equivalent to the additional distance the signal must travel to reach the farther collector. That additional path length is given by,

$$
\Delta p=b \sin \theta
$$


where $b$ is the separation between the collectors and $\theta$ is the angle between the wavefront and the line on which the two collectors lie. That simple configuration however only allows one data point of the spatial Fourier transform of the image to be acquired. It is referred to as a point in the $u-v$ plane and is determined by,

$$
\begin{aligned}
& u= \pm \frac{x_{i}-x_{j}}{\lambda} \\
& v= \pm \frac{y_{i}-y_{j}}{\lambda}
\end{aligned}
$$

where $\left(x_{i}, y_{i}\right)$ and $\left(x_{j}, y_{j}\right)$ are the spatial coordinates of the $i^{\text {th }}$ and $j^{\text {th }}$ apertures and $\lambda$ is the wavelength of the signal source. The variables $u$ and $v$ therefore represent the spatial wave numbers of intensity variations across the image. In order to get better resolution, image data should be sampled from several u-v plane positions, which can be achieved by moving the apertures nearer and farther from each other.

\subsection{Space Technology 3 (ST-3)}

\subsubsection{Overview}

[Blackwood, 1999], [Blackwood, 1998], [Lau, 1999], [ST-3 Web Site, 2001]

ST-3 is a demonstration mission for formation flying and optical interferometry consisting of two satellites, scheduled for launch aboard a Delta 7325 rocket in 2005, into a heliocentric orbit trailing the Earth. The demonstration of separated spacecraft optical interferometry will be an enabling technology for Terrestrial Planet Finder (TPF), which will be discussed in more detail in Section 2.3, and much of the hardware and software elements on ST-3 will help build confidence for the Space Interferometry Mission (SIM).

At the beginning of the mission, Space Technology 3's two spacecraft will be structurally connected. During this time the spacecraft will test out all interferometry systems as a single, fixed baseline Michelson interferometer. After this first stage of testing, the truss that ties the two spacecraft together will be separated, readying the spacecraft for the next 
experimental mode. The spacecraft will then perform formation flying maneuvers at relative spacecraft distances from 50 meters to 1 kilometer. They will then go through a series of maneuvers to test their formation flying sensors and controls. The distance between them will be expanded and contracted and they will be rotated as a formation .

Once this has been demonstrated, Space Technology 3 will enter the final stage of its sixmonth mission. The interferometer mode will be turned back on and the spacecraft will be flown in formation to gather scientific data. ST-3 will image bright astrophysical objects (14th magnitude and brighter) in the visible at 0.55 to 0.9 microns. Among the data that ST-3 may collect are images of three kinds of stars: Be stars (hot stars that have a disk of hot gas surrounding them), Wolf-Rayet stars (hot stars with a very heavy outflow of gas), and M-dwarf stars (red stars, substantially cooler than the Sun).

During the interferometer phase of the mission, the two satellites will fly in formation, with one satellite (a collector/combiner) remaining at the focus of a virtual paraboloid and the other (a collector) moving to various positions along the paraboloid's surface in order to sample several different optical baselines, up to 200 meters. A schematic of this operational mode is shown in Figure 2.2. The telescopes in both spacecraft will capture light from distant stars which will be combined. The data will then be sent to Earth, where, using computer synthesis, images will be formed.

\subsubsection{Given Requirements}

The ST-3 mission will be designed for imaging approximately 50 target stars during its six month lifetime. The expected position accuracy requirement is approximately $\pm 5 \mathrm{~cm}$. The rest of the position stabilization is assumed to be handled by payload components including optical delay lines, piezo-electric actuators and voice coils. In addition, maximum drift speeds of $.2 \mathrm{~mm} / \mathrm{s}$ and 'no thrusting' periods of over 1200 seconds are required, while remaining inside the deadband during imaging. 


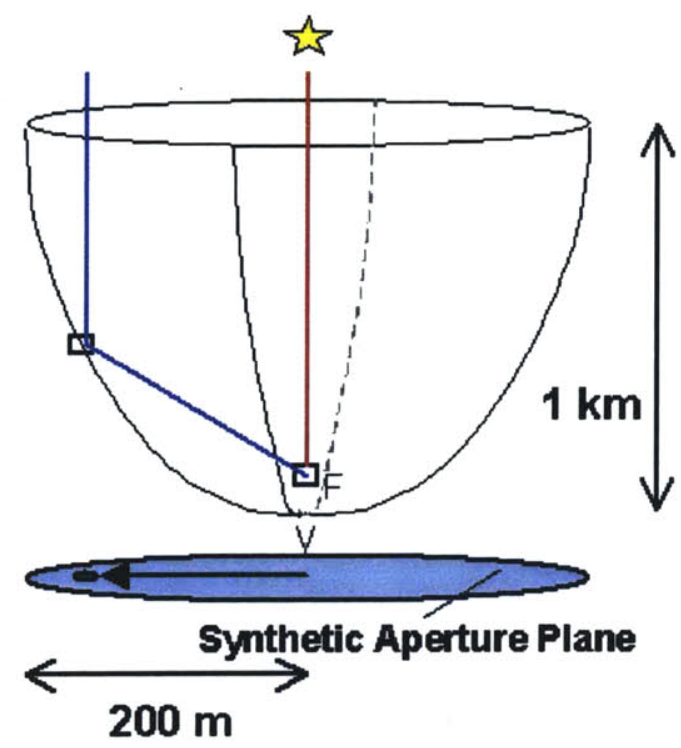

Figure 2.2 Schematic of ST-3 interferometry phase showing the collector spacecraft on the surface of a paraboloid whose focus is fixed at the combiner spacecraft [Blackwood, 1999].

\subsection{Terrestrial Planet Finder (TPF)}

\subsubsection{Overview}

[Beichman, 1997-1998] \& [Beichman, 1999]

Terrestrial Planet Finder is an optical interferometer mission planned to be launched in 2011 into an earth-trailing or the Earth-Sun L2 (Langrangian neutrally stable) point heliocentric orbit. The operational lifetime is slated for five years or more. During this time, it will study many aspects of planets including: their formation and development in disks of dust and gas around newly forming stars; the presence and features of those planets orbiting the nearest stars; their numbers at various sizes and places; and their suitability for sustaining life. Combining the high sensitivity of a space telescope with the highly detailed pictures of an interferometer, TPF will be able to reduce the glare of parent stars by a factor of more than one hundred-thousand, enabling imaging of planetary systems up to 50 light years away. The science mission has a goal of surveying 150 stars for evidence of planets, 50 of which will subsequently be spectroscopically characterized. Of these, the 
five star systems with the most promising evidence of habitable planets will be observed with highly sensitive spectroscopy. In addition to measuring the size, temperature, and placement of planets as small as the Earth in the habitable zones of distant solar systems, TPF's spectroscopic capability will allow the use of data concerning the relative amounts of gases like carbon dioxide, water vapor, ozone and methane to determine if a planet could support life. In addition to studying planets around nearby, mature stars like the sun, TPF will advance the understanding of how planets and their parent stars form. The disks of forming stars are a few astronomical units (AU) across. TPF will study structures on the scale of a few tenths of an AU to investigate how both gaseous and rocky planets form out of disk material. TPF will investigate whether rocky planets form in warmer regions and gaseous planets in colder regions, as theory predicts. Figure 2.3 shows a picture of what the system might look like while imaging a distant planetary system.

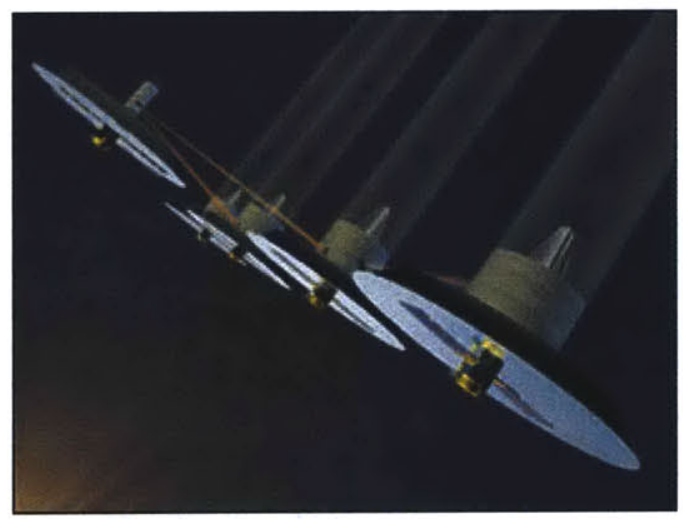

Figure 2.3 Artists rendition of four TPF spacecraft flying in formation, observing a distant solar system (white = starlight, red = metrology). [Beichman, 1997-1998]

This design is based on an example concept, as delineated in Table 2.1.

The above concept requires the spacecraft to be divided into a hot side and a cold side. The optics need to be maintained at $35 \mathrm{~K}$ while all of the other standard spacecraft subsystems are to be kept near the standard temperature of $300 \mathrm{~K}$. Therefore, the design 
TABLE 2.1 TPF example concept design overview [Beichman, 1997-1998].

\begin{tabular}{|c|c|c|c|c|c|c|}
\hline Telescopes & $\begin{array}{l}\text { Base- } \\
\text { line }\end{array}$ & $\begin{array}{c}\text { Angular } \\
\text { Resolution }\end{array}$ & $\begin{array}{l}\text { Field } \\
\text { of view }\end{array}$ & $\begin{array}{c}\text { Wavelength } \\
\text { Range }\end{array}$ & $\begin{array}{c}\text { Spectral } \\
\text { Resolution }\end{array}$ & Sensitivity \\
\hline $\begin{array}{c}4 @ 3.5 m \\
\text { Dia }\end{array}$ & $\begin{array}{c}75- \\
1000 \mathrm{~m}\end{array}$ & $\begin{array}{c}7.5 \times 10^{-4} \\
\text { arcseconds } \\
\text { (as) }\end{array}$ & $\begin{array}{c}0.25 \text { as } \\
\text { at } 3 \mu \mathrm{m} \\
1.0 \text { as } \\
\text { at } \\
12 \mu \mathrm{m}\end{array}$ & $\begin{array}{c}7-20 \mu \mathrm{m} \\
\text { planet } \\
\text { detection } \\
3-30 \mu \mathrm{m} \\
\text { general } \\
\text { imaging }\end{array}$ & $\begin{array}{c}\mathrm{R}=\lambda / \Delta \lambda \sim 20 \\
\text { planet } \\
\text { detection }\end{array}$ & $\begin{array}{c}0.35 \mu \mathrm{Jy} \\
\text { at } 12 \mu \mathrm{m} \\
\left(5 \sigma \text { in } 10^{4}\right. \\
\mathrm{s} \text { at } \mathrm{R} \sim 3)\end{array}$ \\
\hline
\end{tabular}

includes the large white discs shown in Figure 2.3, which act as sun shades for the optical components.

\subsubsection{Given Requirements}

Minimum spacecraft separations of approximately 10 meters and control sensitivities on the order of a millimeter are required. The Autonomous Formation Flying (AFF) system for the TPF constellation must provide relative range, range rate, and elevation bearings between spacecraft over the range 10 to 1000 meters for controlled maneuvers. In addition, relative position knowledge must be determined to $\pm 1 \mathrm{~cm}$, relative velocity to \pm 0.1 $\mathrm{mm} / \mathrm{s}$ and attitude knowledge to \pm 1 arcmin. The corresponding position control requirement is expected to be $\pm 5 \mathrm{~cm}$. Also, independent six degree-of-freedom attitude and translation control is necessary.

\subsection{Laser Interferometry Space Antenna (LISA)}

\subsubsection{Overview}

[Bender, 1998], [Phinney, 2000] \& [Folkner, 1998]

The primary objective of the LISA mission is to detect and observe gravitational waves from massive black holes and galactic binary stars in the frequency range between $10^{-4}$ to $10^{-1} \mathrm{~Hz}$. Useful measurements in this frequency range cannot be made on the ground 
because of the unshieldable background of local gravitational noise and an inability to make interferometers with large enough baselines.

LISA consists of three identical spacecraft, launched from a Delta-II $7925 \mathrm{H}$ rocket, which form an equilateral triangle with sides of five million kilometers in length and a spacecraft at each vertex once deployed. The entire formation orbits the sun at $1 \mathrm{AU}$, trailing the earth's orbit by $20^{\circ}$. The plane of the triangle formed by the spacecraft is inclined at an angle of $60^{\circ}$ to the plane of the ecliptic. As the formation orbits the sun during the course of a year, it rotates somewhat like a rolling wheel so that the spacecraft positions and attitudes change relative to sun. An illustration of the orbit can be found in Figure 2.4. This

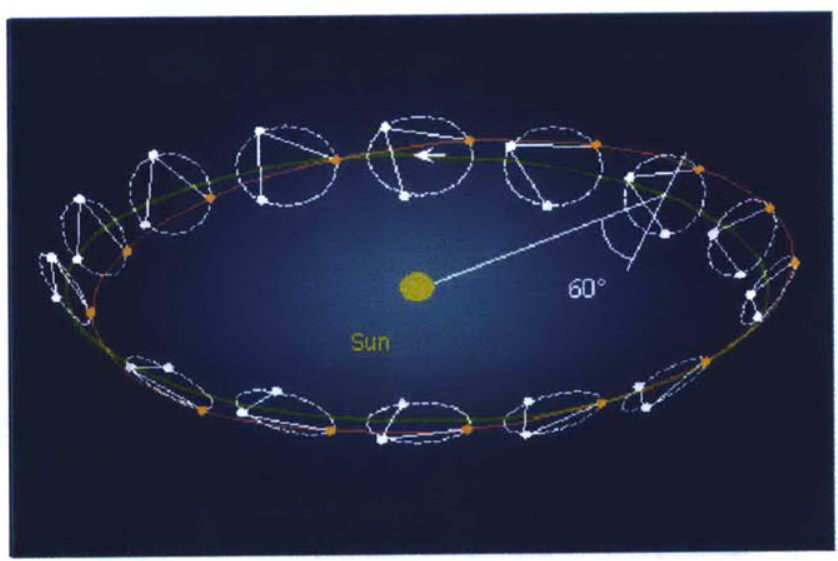

Figure 2.4 Illustration of LISA formation movement during an orbit. The green line represents the orbit of the earth (in the plane of the ecliptic) and the red line is the orbit of one LISA spacecraft (orange dot) [Bender, 1998].

variation allows for the determination of the direction of the gravity wave sources.

To detect the presence of gravitational waves, two laser beams, which are phase-locked together are emitted from each spacecraft toward the other two. These phase-locked lasers behave like a laser whose beam has been split, but without decreasing the intensity of each resultant beam and creating a system with higher redundancy and reliability. Each of the spacecraft tracks the spacecraft at the other two distant vertices. A designated central 
spacecraft corresponds to the central mirror of a Michelson interferometer, while the two distant spacecraft correspond to the two end mirrors. When a gravity wave passes through the system it causes a strain distortion in space which will be detected by measuring the fluctuations in separation between proof masses inside the different spacecraft. The separated masses constitute the arms of the interferometer. The measurements are performed by optical interferometry which determines the phase shift of laser light transmitted between 'free-floating' test masses. These 'free floating' test masses are so called because they will be following an orbit influenced only by the sun's gravity. The spacecraft acts as a shield against any disturbing forces like solar radiation pressure and solar wind pressure. Figure 2.5 is a diagram of one spacecraft, showing the optical bench with the free-floating test mass (in yellow) and the phase locked lasers (beams in red).

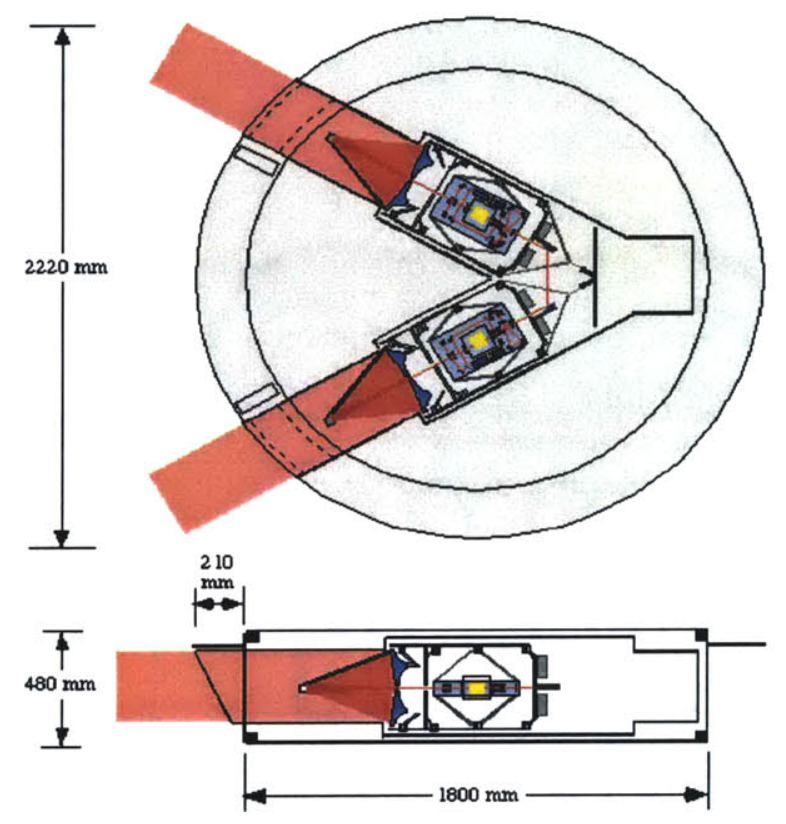

Figure 2.5 LISA spacecraft payload diagram. Gold squares are proof masses, shades of red represent laser path [Folkner, 1998]. 


\subsubsection{Given Requirements}

The main requirements on spacecraft drag-free and attitude control are derived from payload constraints. The propulsion and control systems must force the spacecraft to follow the test mass to $1 \mathrm{~nm} / \mathrm{Hz}^{1 / 2}$. The acceleration noise on the test mass must not exceed 1 $\times 10^{-15} \mathrm{~ms}^{-2} \mathrm{rms}$ in the band from $10^{-4}$ to $10^{-1} \mathrm{~Hz}$. The control signals are derived from the payload-provided electrostatic accelerometer (the test mass, surrounded by relative displacement electrostatic sensors). The attitude control system points each spacecraft toward the spacecraft at the other end of its optical path. The pointing tolerance is about $5 \mathrm{nrad} /$ $\mathrm{Hz}^{1 / 2}$ for frequencies above about $10^{-4} \mathrm{~Hz}$ and $30 \mathrm{nrad}$ for lower frequencies and DC. The mission is nominally slated for a two year duration with an extended design option that would allow a ten year operational lifetime.

\subsection{Micro Arcsecond X-ray Imaging Mission (MAXIM)}

\subsubsection{Overview}

[MAXIM Web Site, 2001] \& [Cash, 2000]

MAXIM is a proposed X-ray interferometry mission with the goal of imaging massive black hole event horizons down to an angular resolution of 0.1 to $1 \mu$-arcseconds. The best candidate black hole to observe is the nearby active galaxy M87. This is believed to harbor a 100 million solar-mass black hole at a distance of approximately 1 million parsecs. An angular scale of micro arc-seconds is required to resolve the event horizon of the supermassive black hole in M87 (the currently known stellar mass black holes in our galaxy have an angular scale several orders of magnitude less than this and would be even more challenging to observe). This mission would have 100,000 times better angular resolution than the current capability of $x$-ray optics (as defined by the Chandra $x$-ray optic).

Dr. Webster Cash at University of Colorado and his colleagues have achieved 0.1 arcsecond resolution in the laboratory with their design of an X-ray interferometer. This resolution is comparable to Hubble. In general, X-ray telescopes are difficult to build because, 
to obtain a true focus, X-ray photons must reflect twice from very precisely configured hyperbolic and parabolic surfaces. The Chandra facility achieves a 0.5 arcsecond resolution, which is a rather impressive feat in itself.

However the need for smooth mirrors, normally a disadvantage for $\mathrm{x}$-ray astronomy, is an advantage when doing interferometry. Instead of precisely focusing $x$-rays with expensive mirrors onto a detector, readily-made flat mirrors can be used to mix the x-ray wavefronts. This interferometric technique can produce an even sharper image because of the amplified fringes produced. The very nature of x-rays being difficult to focus means that they can be mixed more easily than visible light and radio waves.

Much of the technology necessary to accomplish this goal is yet to be developed. The mission itself is not expected to be feasible until approximately 2025. The mission is expected to be situated in a heliocentric orbit. The most recently proposed system architecture available is based on a constellation of 32 spacecraft around the perimeter of a circle with a diameter of approximately 200 meters, with one spacecraft at the center. Ten kilometers behind this 'wheel' a converger spacecraft combines the collected x-ray signals and focuses them onto the detector. The detector spacecraft flies $5000 \mathrm{~km}$ behind this circular constellation and relays the information back to Earth. A pictorial representation of this configuration can be seen in Figure 2.6. Baselines, and therefore spacecraft separations, from 100 to 1000 meters are expected to be covered by the interferometer. This will provide the ability to image with 100 nano-arcsecond resolution. With 100 micro-arcsecond resolution, astronomers could image the coronae of nearby stars, seeing the actual disks of other stars which appear now only as points of light. With 300 nano-arcsecond resolution, astronomers could image a black hole.

\subsubsection{Given Requirements}

Based on the concept and framework for the mission as it exists now, it is assumed that, nominally, 20 nanometer position and 0.1 milli-arcsecond attitude stability will be necessary to provide the angular resolution desired for imaging the event horizons and other 


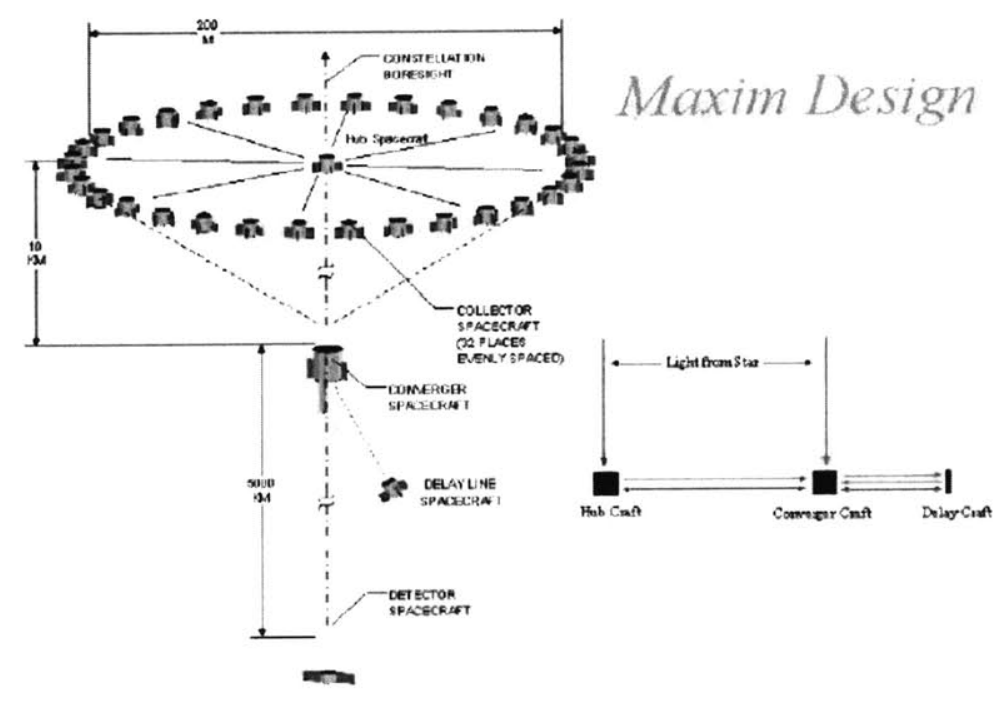

Figure 2.6 Illustration of fully deployed MAXIM formation including 33 collectors, a combiner, a delay line and a detector spacecraft [Cash, 2000].

stellar objects of interest. However, the position maintenance in the axial direction, with respect to the center (hub) spacecraft, may need to have a stability of approximately 1 nanometer. These requirements come from the MAXIM world wide web page as excerpted in Figure 2.7 and from a MAXIM preliminary design document [Cash, 2000]. In addition, some requirements for the attitude control and telemetry systems are specified including angular and position knowledge at levels of $3 \times 10^{-8}$ arcseconds and 2 nanometers, respectively.

There are two major disturbance sources that are expected to affect the ability of the spacecraft to maintain their formation. The first is solar radiation pressure which is expected to be on the order of 5 to 9 micronewtons per square meter, depending on the reflectance of the particular spacecraft. In addition, there is a residual gravitational force imparted by the sun because the collector and detector spacecraft operate in non-Keplerian orbits. These orbits were chosen so that the more massive converger spacecraft would follow a force free, true Keplerian orbit. The gravitational acceleration due to non-Keplerian orbits is expected be approximately of $2 \times 10^{-7}$ meters per second squared, perpendicular 


\begin{tabular}{|c|c|c|c|c|c|c|c|c|}
\hline \multicolumn{9}{|c|}{ X-ray Interferometer I olera nces } \\
\hline $\begin{array}{l}\text { Resolution } \\
\text { Areseconds }\end{array}$ & 1 & 0.1 & $10^{-2}$ & $10^{-3}$ & $10^{-4}$ & $10^{-5}$ & $10^{-6}$ & $10^{-7}$ \\
\hline Baseline (m) & & & & & 1 & 10 & 100 & 1000 \\
\hline $\begin{array}{l}\text { Mirror Length } \\
\text { (m) }\end{array}$ & 0.1 & 0.1 & 0.3 & 3 & 3 & 3 & 3 & 3 \\
\hline $\begin{array}{l}\text { Position } \\
\text { Stabililty (nm) }\end{array}$ & 200 & 20 & 20 & 20 & 20 & 20 & 20 & 20 \\
\hline $\begin{array}{l}\text { Angular Stability } \\
\text { (arcsec) }\end{array}$ & 50 & 10 & 2 & 0.3 & 0.1 & 0.01 & $10^{3}$ & $10^{-3}$ \\
\hline Figure & $\lambda / 5$ & $\lambda / 20$ & $\lambda / 50$ & $\lambda / 100$ & 7. 100 & $\lambda / 200$ & 7. 200 & $\lambda / 200$ \\
\hline $\begin{array}{l}\text { Polish } \\
\text { (Angstrom rms) }\end{array}$ & 50 & 30 & 20 & 20 & 20 & 20 & 20 & 20 \\
\hline $\begin{array}{l}\text { Angular : } \\
\text { Knowledge (as) }\end{array}$ & 0.3 & 0.03 & $3 \times 10^{-3}$ & $3 \times 10^{-4}$ & $3 \times 10 r^{5}$ & $3 \times 10^{-6}$ & $3 \times 10^{-7}$ & $3 \times 10^{-8}$ \\
\hline $\begin{array}{l}\text { Position } \\
\text { Knowledge (nm) }\end{array}$ & & & & & 2 & 2 & 2 & 2 \\
\hline E/ $\Delta \mathrm{E}$ Detector & & & & & 10 & 20 & 20 & 20 \\
\hline \multicolumn{9}{|l|}{ Pathfinder } \\
\hline $\begin{array}{l}\text { Major } \\
\text { Observatory }\end{array}$ & & & & & & & & \\
\hline
\end{tabular}

Figure 2.7 Baseline requirements for the MAXIM micro-arcsecond Xray interferometer shown in the cells shaded green [Cash (Web Site), 2000].

to the plane of the ecliptic. With the assumption that the detector would have a nominal distance of 5000 kilometers from the plane of the ecliptic and the collector 10 kilometers, the nominal thrust levels required for the two spacecraft to cancel this gravitational perturbation would be 200 micronewtons and 2 micronewtons, respectively.

\subsection{Submillimeter Probe of the Evolution of Cosmic Structure (SPECS)}

\subsubsection{Overview}

[Shao, 2000], [Mather,1998], [Leisawitz, 2000], [Leisawitz (Web site), 2000]

The primary goal of SPECS is to provide a definitive observational basis for understanding the history of and the processes that drive the development of complex structure from the homogeneous early universe. The SPECS concept, slated for launch into the L2 orbit 
(Earth-Sun Lagrangian neutrally stable orbit) sometime during the 2020s, is to develop an interferometer that will detect in the submillimeter wavelength range with high resolution, allowing an in-depth view of nearly half of the total luminosity and ninety-eight percent of the post-Big Bang photons in the universe. Figure 2.8 shows the intensity of submillimeter photons as compared to those in the infrared and optical range.

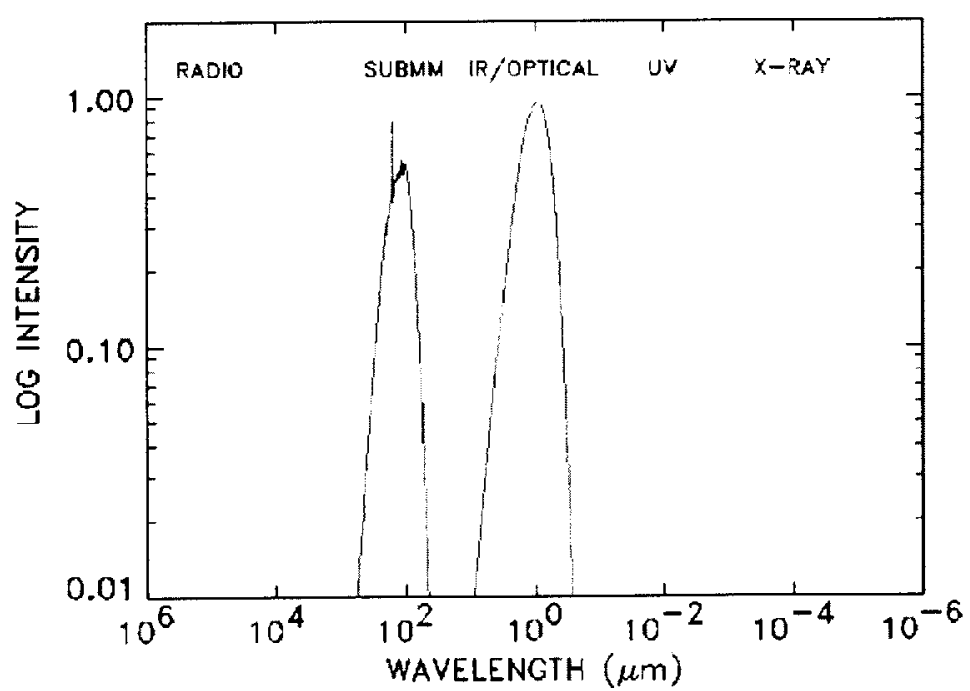

Figure 2.8 Post-big bang photon intensity plotted on a log scale showing the significance of surveying the universe in the submillimeter wavelengths [Leisawitz, 2000].

The configuration assumed for this mission is a set of 3 mirrors tethered to a central hexagonal structure with radial trusses that contain beam combining optics and delay lines. The tethers are variable in length and each mirror has a counterweight (or mirror) on the other end of its tether to reduce spin-up as the mirrors are drawn in toward the center. The entire formation will rotate about the line of sight. A drawing of the deployed formation with counterweights is shown in Figure 2.9. In order to scan the u-v (synthetic aperture) plane, the tethers are lengthened and shortened to create all of the necessary baselines. 


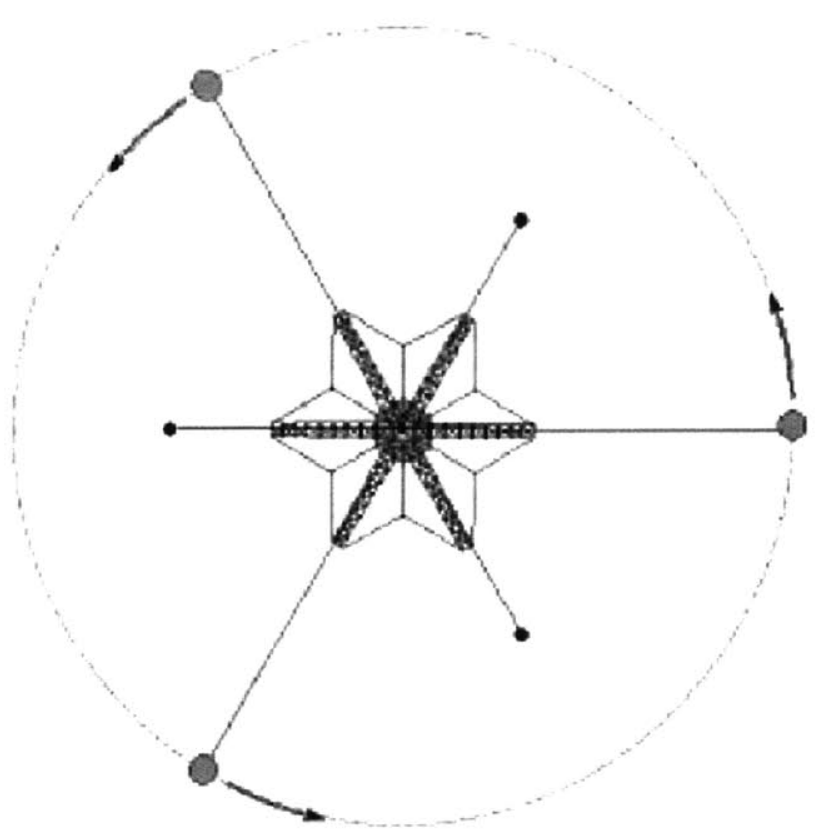

Figure 2.9 Tethered three mirror design schematic of SPECS spacecraft during imaging from the target's perspective [Leisawitz, 2000].

\subsubsection{Given Requirements}

In order to maintain the desired angular resolution and sensitivity, the metrology system is required to be accurate to approximately one micron in translation and fifty milliarcseconds in pointing. Depending on the number of systems between the optical delay lines and the spacecraft actuation, the propulsion system may need to be able to provide thrust authority increments precise enough to maintain position to hundredths of millimeters if not several microns. Some of the characteristic design parameters presented for the SPECS system are shown in Table 2.2. The specifications therein provide for a system

TABLE 2.2 SPECS design parameters [Leisawitz (Web site), 2000]

\begin{tabular}{|c|c|}
\hline Telescopes & $3,4 \mathrm{~m}$ diameter apertures \\
\hline Telescope Temperature & $4 \mathrm{~K}$ \\
\hline Maximum Baseline & $1 \mathrm{~km}$ \\
\hline Detectors & 6,100 X 100 pixel arrays \\
\hline Detector Type & Superconducting Tunnel Junction or bolometer \\
\hline
\end{tabular}


TABLE 2.2 SPECS design parameters [Leisawitz (Web site), 2000]

\begin{tabular}{|c|c|}
\hline Spectrometer & Michelson Interferometer \\
\hline Wavelength & $40-500 \mu \mathrm{m}$ \\
\hline Spectral Resolution & up to $10^{4} \mathrm{~Hz}$ \\
\hline Angular Resolution & 0.06 arcsec at $300 \mu \mathrm{m}$ \\
\hline Field of View & $3.4 \operatorname{arcmin}$ \\
\hline Typical Exposure & $1 \times 10^{5} \mathrm{~s}$ \\
\hline Typical Sensitivity, $v \mathrm{~S}_{v}(1 \sigma)$ & $0.3-1 \times 10^{-19} \mathrm{~W} / \mathrm{m}^{2}$ \\
\hline
\end{tabular}

that will be capable of characterizing the first stars to form, learn when they first produced heavy elements and determine when the first dust formed. 


\section{Chapter 3}

\section{METRICS FOR PROPULSION SYSTEM SELECTION}

This chapter defines and describes the measures which are used to evaluate the impact of the different propulsion systems on the missions. The framework for constructing the following metrics is interpreted from the Generalized Information Network Analysis (GINA) Methodology developed by Shaw, et al. [Shaw, 1998]. As outlined in Section 1.4, this methodology aims at evaluating and designing missions and subsystems with a view to maximizing the high level performance of the mission.

\subsection{Overview of the Generalized Information Network Analysis (GINA) Metrics}

\subsubsection{Isolation}

A system's ability to discern and isolate different signal sources from a general field of view is often a critical mission driver. For imaging systems, different sources can be identified by detecting in different frequency bands and spatially separated sources can be isolated using a high resolution detector. An aperture can distinguish sources separated by a a distance that is at least as large as the resolution of the detector.

\subsubsection{Integrity}

The integrity measures the error performance of the system. "The integrity is most commonly represented by the probability of making an error in the interpretation of a signal 
based on noisy observations" [Shaw, 1998]. In order to determine what information a sensor is collecting, it uses an observation of the signal plus the noise. Therefore, the probability of making an error in the interpretation of the information is a function of the energy in the signal. The integrity of an imaging system could be measured, for example, by the pixel error density of an image. Accordingly, the integrity metric is related to the signalto-noise ratio (SNR); a larger SNR results in an augmented integrity metric, however it is measured.

\subsubsection{Rate}

The rate metric is simply a measure of the inverse of the length of time required to deliver one unit of the product or service that is being supplied to the customer. For imaging systems it will depend greatly on how long it takes for a target to be acquired (put in view of the sensor) and the amount of time needed for the data to be gathered from the target. There also may be a contribution from data transfer rates if image data needs to be dumped in order to continue at various points in the mission.

\subsubsection{Availability}

Availability is defined as the probability that the other metrics will meet or exceed their requirements at a given time. The availability is a measure of the mean and variance of the isolation, rate and integrity supportable by the system. In a somewhat more simplistic view of the availability metric, it is closely related to the percentage of the total mission lifetime that is actually spent collecting data.

\subsubsection{Cost Per Function}

Just as in all financially constrained projects, cost becomes the main high-level metric by which to measure performance. In this case, the total lifetime cost for a mission, normalized by the number of products delivered for that price, is called the cost per function (CPF) and is used for the high level architecture comparison. 
In order to make estimates of this metric it is necessary to have some way of approximating overall mission cost, with some sensitivity to minor changes in subsystems, and the amount of useful data. Both of these things require an estimate of the lifetime of the mission. And, in order to make an assessment of the number of products delivered, it is necessary to compute a rate of production or delivery or to determine the time required to create and deliver one such product. For an optical interferometry mission this amounts to the number of years of the mission life and the time required to sense and transmit one picture. Total mission cost accounts for all hardware, operations, maintenance, launch and development costs.

\subsection{Interpretation and Application of GINA Metrics}

The goal of using the previous metrics as a framework for this study is to determine which micropropulsion systems should be used to best satisfy overall requirements of each of the considered missions. It is important that these metrics be quantitative and applicable to all the missions and propulsion systems of interest. Additionally, these metrics are designed to determine which propulsion system allows a given mission's requirements to be met with the highest performance, not to make an absolute decision about which is the best propulsion system.

Given detailed mission information and the ability to interactively work with the customer in interpreting and designing a mission around the requirements, all of the GINA metrics can be used as a complete set. This is a powerful tool for creating optimal spacecraft and overall architecture designs for each mission. However, with a desire to investigate five future missions, all with technology development-requiring components, and the effects of four different propulsion systems (of which, two have never been flown) on them, it is necessary to scale back the use and interpretation of the GINA metrics.

In order to address high level mission requirements, the metrics used in this study are designed to account for indirect trade effects wherever they significantly impact the mission's capabilities. An example of such an effect includes the increased mass of the power 
processing unit required to provide higher power or faster pulsing capabilities so that more impulse can be applied to the spacecraft by the propulsion system in a given time, which would allow the mission to be carried out more rapidly. Therefore, propulsion system masses should not simply be thruster and propellant masses, but should include power processing unit masses, tankage and other additional structural necessities unique to each system.

For the work presented in this document, the GINA metrics have been pared and consolidated into two main categories. The first is integrity, which is defined in a way similar to the original sense in which Shaw dealt with it, though somewhat simplified. The second is related to the cost per function metric, but does not actually have the goal of quoting dollar values, but rather masses and technology development extent estimates that have very strong influences on total mission cost, mainly in hardware and research and development costs. The other three metrics described in Section 3.1 are used primarily as requirements used in formulating the designs, instead of as performance metrics.

\subsection{Metrics Used for Propulsion System Evaluation}

\subsubsection{Integrity}

In the context of missions whose science objectives employ interferometry to acquire a signal, the integrity metric can be affected by anything that degrades the signal quality. Signal quality, in these instances, depends not only on the gathering of the original signals from the source, but on the reliability with which those signals can be combined to reconstruct a coherent image. Therefore, from the point of view of designing the propulsion system, the integrity metric can generally be thought of as how well the spacecraft's position and attitude can be maintained. This kind of stationkeeping or formation flying accuracy often determines the level of confidence that can be attributed to the data being gathered. 
One way to measure a spacecraft's formation flying precision is to compute the power spectral density (PSD) of its position error. The position error is simply the difference of the desired position of the spacecraft as a function of time and the actual position of the spacecraft as a function of time. This PSD of the position error gives information about the amount of position noise power in the system, as a function of frequency. In general the PSD of a signal $x(t)$ is defined as:

$$
\operatorname{PSD}(\omega)=\frac{1}{2 \pi} \int_{-\infty}^{\infty} R(\tau) e^{-i \omega \tau} d \tau
$$

where $R(\tau)$ is the autocorrelation function of $x(t)$ :

$$
R(\tau)=E[X(t) X(t+\tau)]=\langle x(t+\tau, k) x(t, k)\rangle
$$

Additionally, a similar analysis can be performed on the acceleration experienced by the spacecraft by simply substituting $\ddot{x}(t)$ for $x(t)$ in the equations above. This analysis can often be very useful for missions in which disturbances in a certain frequency band need to be kept to specified limits because of the kind of signal being observed. But, even for a mission that does not have requirements specified in terms of power spectra, it can be a useful metric. The PSD of the position signal impacts the integrity of the data by affecting its signal-to-noise ratio (SNR). The SNR is defined as:

$$
S N R=\frac{P_{t \operatorname{arget}}}{P_{\text {noise }}}
$$

where the noise power, $\mathrm{P}_{\text {noise }}$, is defined by the following:

$$
P_{\text {noise }}^{2}=\sum_{n=\text { source }} P_{n}^{2}
$$

The integral of the PSD over the frequency band in which the detector is observing signals is the total average noise power added by the particular noise source. The greater this value, the lower the SNR. If it is desired that the SNR be kept at a specified level, it will 
take longer to image a target when the magnitude of the PSD over the relevant bandwidth is greater.

Different propulsion systems' noise PSDs have different origins. This is a means of differentiating pulsed from continuous thrusting schemes. For pulsed systems the main source of noise power comes from the pulsing itself. The non-smooth acceleration and velocity profiles created by impulsive thrusting have a broad-band spectrum, with most power concentrated at the frequency of the pulsing. Continuously varied thrusting systems do not suffer from this problem as much because they create smoother thrusting profiles with smaller trajectory errors. The main sources of noise from continuous thrusters are the inherent variability in the physical processes that provide the thrust and the fineness/coarseness with which their thrust levels can be incremented and the performance of the controller. In order to determine the characteristics of noise from physical processes these thrusters might produce, test data are necessary.

In many cases, an analysis as detailed as characterizing the power spectrum of the noise in the spacecraft's rigid body dynamics is not necessary. In those cases, enough information may be provided by specifying the minimum dead-band width to which a propulsion system can keep the spacecraft, as is described in more detail in Chapter 4. In this work deadband width will be treated largely as a requirement dictated by the customer. However, in some missions and propulsion system contexts it will be useful as an integrity related metric as well.

\subsubsection{Cost}

When conducting a full scale trade study to determine the very highest level of system definition and architecture the cost per function metric, as described in Section 3.1.5, is likely to be the best metric for determining overall performance. However, when all the necessary information is not available or is too much to be assembled in a given time frame a similar but more sparing substitute may be more desirable. Such is believed to be the case in this study. 
As a result, the impact that a particular propulsion system design will have on the overall system will be measured indirectly, by way of two main attributes. The first is a mass value, which takes into account the effects of actual propulsion system mass and relative changes in the mass of other spacecraft components caused by the propulsion system design. The other attribute is a technology readiness level rating as defined in the standardized system used by NASA. This rating indicates how close a given technology is to maturity, in the sense of space flight readiness.

To account for all of the components contributing to the mass, hardware associated directly with the propulsion system will be taken into account. This includes the structure of the thruster itself, any specialized electronics required for the thruster, the propellant and the feed system necessary to supply the propellant to the thruster. In addition, mass contributions from the power system of the spacecraft needed to support the propulsion system are included. The main components that contribute mass in this category are solar arrays and batteries. Fractional increases in the masses of these components because of the various power demands of the particular propulsion systems can be added to augment the mass metric, in effect penalizing propulsion systems for demanding more power.

For the purpose of creating a single cost metric for each mission, a dollar value is attributed to each kilogram of hardware. The dollar value is based on both launch costs and hardware manufacturing costs. The launch cost is calculated from the following equation,

$$
\text { Cost }_{\text {Launch }}=(\$ 40,000) \times(1.328) \times m_{\text {total }}
$$

where the dollar per kilogram value comes from an average of launch cost data found in [Larson, 1992] and the numerical factor (1.328) accounts for the inflation rate between 1992 dollars and 2001 dollars. The hardware manufacturing costs are based on theoretical first unit costs (TFUs) that are described in [Larson, 1992] as function of hardware mass. Subsequent units (after the first unit) have a price discounted by a learning curve equation. The TFU cost equation is given as, 


$$
\operatorname{Cost}_{\mathrm{TFU}}=1.328\left(186000\left(m_{\mathrm{total}}-m_{\mathrm{prop}}\right)^{0.73}-364000\right)
$$

and the total cost of $\mathrm{N}$ units is calculated from

$$
\operatorname{Cost}_{\text {hardware }}=\operatorname{Cost}_{\mathrm{TFU}} N^{B}
$$

where the exponent $B$ is given by the learning curve equation with slope $S$ :

$$
B=1-\frac{\ln \left(\frac{100 \%}{S}\right)}{\ln 2}
$$

This enables the technology readiness level and the effective propulsion system mass to be combined into a single metric that indicates the relative cost of the various propulsion systems for each mission.

The technology readiness ratings aim to roughly quantify the cost of performing the research and development activities necessary to bring a propulsion system to the state of maturity necessary for it to fly on a space mission as part of the spacecraft that supports the payload, not as a payload in and of itself, with the goal of further characterizing and verifying its own performance. The basic descriptions of the nine levels of readiness are listed in Table 3.1 [Mankins, 1995]. The cost associated with achieving each of these readiness levels can differ significantly depending on the technology and its intended application. Mankins gives some basic, qualitative guidelines as to the relative costs of advancing from one technology readiness level (TRL) to the next, but in order to use them in conjunction with the mass metrics described above, some kind of cost estimates, if only rough, need to be applied to them.

In order to construct technology development cost estimates, information from Mankins' memo and a recent NASA Technology Announcement [Spencer, 2000] were combined to create a relationship between the current TRL of a technology and the amount of money in current dollars (without accounting for the time value of money) required to bring the 
TABLE 3.1 NASA Technology Readiness Levels (TRLs) [Mankins, 1995]

\begin{tabular}{|c|l|}
\hline TRL & \multicolumn{1}{|c|}{ Description } \\
\hline \hline 1 & Basic principles observed and reported \\
2 & Technology concept and/or application formulated \\
3 & Analytical and experimental critical function and/or characteristic \\
4 & proof-of-concept achieved in a laboratory environment \\
5 & Component and/or breadboard validated in a laboratory environment \\
6 & Component and/or breadboard validated in a relevant environment \\
7 & System/subsystem model or prototype demonstrated in a relevant \\
8 & System/subsystem prototype demonstrated in a space environment \\
9 & Actual system completed and flight qualified through test and dem- \\
\hline
\end{tabular}

TRL of the technology through level eight. The development step between level eight and level nine is not included because it is expected that this investment will be necessary for each new mission to which a technology is adapted. The equation is expressed succinctly by the exponential function

$$
\text { Cost }_{\text {development }}=33.4 \times 10^{6}-23700 e^{0.907(\mathrm{TRL})} \text {, }
$$

where Cost $_{\text {development }}$ is in dollars and the qualitative TRL values are as listed in Table 3.1. With each of the TRL descriptions Mankins provides a qualitative estimate of the 'Cost to Achieve' that level, often in terms of the previous levels. These qualitative descriptions were used to create a set of points delineating the relative development costs from level to level of a hypothetical system. This set of relative costs was then fit with an exponential function of the TRL, which has a regression coefficient, $R^{2}=0.98$, so that fractional values of the TRLs can be used in an effort weaken the high sensitivity of the exponential function and provide more accurate cost estimates. Then, using budgets quoted in the Technology Announcement document for developing technologies up to TRL 6 from 4, the hypothetical set of costs was anchored to a real dollar value. While the absolute cost dollar amount may or may not be especially accurate, the trend and, therefore, the relative 
development costs between competing propulsion systems is expected to be meaningful and useful. 


\section{Chapter 4}

\section{PROPULSION SYSTEMS}

The four propulsion systems described in this chapter are the focus of this thesis. For each one, a survey of prior research will be presented along with an explanation of the fundamental physics by which they operate. Following that, an explanation of the way the propulsion system has been modeled for use in this thesis will be presented.

\subsection{Pulsed Plasma Thruster (PPT)}

\subsubsection{Background \& Fundamentals}

The pulsed plasma thruster is an electromagnetic accelerator, which uses solid Teflon (Polytetrafluoroethylene- PTFE) bars as propellant. PPTs have been the subject of research and development efforts since the 1960s and have been flown on several space missions. As the name indicates, they are pulsing thrusters with pulse widths that are characteristically very short, on the order of tens of microseconds. As a result, the minimum amount of impulse that can be imparted to a spacecraft in one pulse (the impulse bit) can be quite small. This precision delivery of impulse is one of the major reasons for the consideration of using PPTs for formation flying satellite missions. In order to give a general idea of the operation regimes in which PPTs have been developed, Table 4.1 is provided. Additionally, there are currently efforts at the Air Force Research Laboratory (AFRL) to build and test PPTs, which have been chosen to fly on the Tech Sat 21 mission, 
TABLE 4.1 Performance data from flight and laboratory PPT testing [Burton, 1998].

\begin{tabular}{|c|c|c|c|c|}
\hline Thruster & $\mathbf{E}_{\mathbf{0}}[\mathbf{J}]$ & $\mathbf{I}_{\mathbf{s p}}[\mathbf{s}]$ & $\mathbf{I}_{\mathbf{b i t}}[\mu \mathbf{N}-\mathbf{s}]$ & $\mathbf{m}_{\text {shot }} / \mathbf{E}_{\mathbf{0}}[\mu \mathbf{g} / \mathbf{J}]$ \\
\hline \hline LES-6 & 1.85 & 300 & 26 & 4.8 \\
\hline LES-8/9 & 20 & 1000 & 297 & 1.5 \\
\hline MIT Lab & 20 & 600 & 454 & 2.8 \\
\hline MIPD-3 & 100 & 1130 & 2250 & 2 \\
\hline Primex-NASA & 43 & 1136 & 737 & 1.5 \\
\hline IL PPT-3 Lab & 7.5 & 600 & 450 & 10 \\
\hline Japan Lab & 30.4 & 423 & 469 & 3.7 \\
\hline China Lab & 23.9 & 990 & 448 & 1.9 \\
\hline
\end{tabular}

with impulse bits of approximately 2 micronewton-seconds and energies per discharge below one Joule [Mueller, 2000].

There are several different configurations of PPTs that have been conceived, many of which have also been tested. The most common varieties are the breech-fed, side-fed, and coaxial versions. For simplicity and generality, attention here will be focused on the breech-fed configuration. Figure 4.1 is a schematic of the breech-fed PPT in cross section.

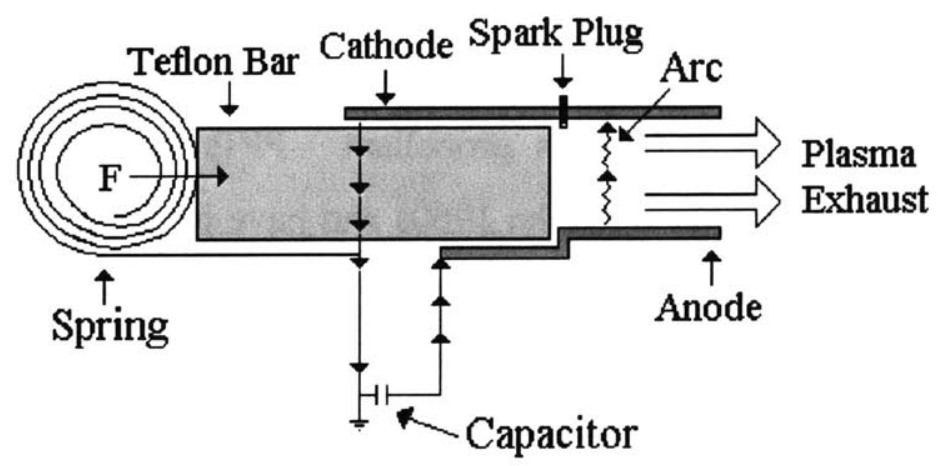

Figure 4.1 Breech-fed pulsed plasma thruster (PPT) schematic

Despite variations in the mechanical design of the thrusters, they all operate according to the same fundamental principles. 
In order to fire a PPT, a capacitor is discharged, creating a large potential across the space between an anode and a cathode. This potential causes a surface breakdown (which is initiated at a semiconducting spark plug surface) on the face of a solid bar of Teflon propellant, ablating it and allowing an arc to pass through the outer, gaseous layer, ionizing it. This arc carries a large current and so induces a magnetic field around itself. Therefore, there is a Lorentz force ( $\mathrm{x}$ B) acting on the ions upstream of the arc (by the right hand rule) that accelerates them downstream. In addition, there is a gas dynamic effect caused by the heating of the ablated Teflon by the arc.

Efforts to quantify the role that each of these acceleration mechanisms play in the overall thruster firing have been made, but detailed, reliable results have been elusive. Early on, William Guman developed an analytic expression for the impulse bit of a breech-fed PPT that represented the contributions from electromagnetic and electrothermal forces [Guman, 1968]:

$$
\begin{aligned}
& I_{b i t}=\frac{1}{2} L^{\prime} \int i^{2} d t+\left[\frac{8(\gamma-1)}{\gamma^{2}(\gamma+1)} m_{\text {shot }} E_{o}\right]^{1 / 2} \\
& L^{\prime}=\frac{\partial L}{\partial x} \\
& L=L^{\prime} x+L_{o}
\end{aligned}
$$

where $L$ is the total inductance of the circuit, $x$ is the length along the nozzle, $L_{0}$ is the inductance of the portion of the circuit outside the nozzle, $i$ is the current, $\gamma$ is the specific heat ratio of the products of decomposition of Teflon $(\sim 1.3)$ and, $\Delta \mathrm{m}$ is the mass ablated and $E_{o}$ is the total energy input to the arc. This equation is only approximate because it assumes that the flow expands at constant area and that all of the energy in the capacitor goes into the arc before mass is ablated [Burton, 1998], which is only true if the pulse length is no more than about one microsecond long.

Much of the research done on PPT performance has been of the empirical sort, which has led to various scaling laws that are somewhat useful for design purposes. One such equa- 
tion, provided by Guman, gives specific impulse as a function of stored energy $\left(\mathrm{E}_{\mathrm{o}}\right)$ and exposed propellant area $\left(A_{p}\right)$ as follows:

$$
I_{s p}=317.5\left(E_{o} / A_{p}\right)^{0.585}
$$

where energy is in Joules and the area is in centimeters squared [Guman, 1975].

From the mechanical standpoint, this thruster is exceedingly simple. It has no moving parts and doesn't need to handle pressurized or flowing fluid propellants. However, physically speaking, the electromagnetic and thermal phenomena involved in the thruster firing are rather complicated. Despite several attempts at creating numerical models, like MACH2 [Turchi, 1999], none have been able to match experimental results in all facets of physical operation. Recent attempts have focused on increasing efficiency, which has been notoriously low (around 10\%), by electromagnetically accelerating all of the ablated Teflon during a given pulse. Much of the Teflon is only thermally accelerated late in the pulse due to indirect heating and never reaches velocities comparable to the electromagnetically accelerated propellant [Mikellides, 1999].

As a result of the pulsed nature of PPTs, the fundamental equations that deal with thruster performance need to be altered slightly. The following essentially shows the discrete analogs to more conventional continuous thruster characteristics [Guman, 1975]. For a continuous thruster scenario, specific impulse is written as:

$$
I_{s p}=\frac{F}{\dot{m} g}
$$

where $\mathrm{F}$ is thrust, $\dot{m}$ is mass flow rate and $\mathrm{g}$ is the gravitational constant on the Earth's surface. In order to apply this kind of metric to a pulsed system, a frequency or time scale must be introduced in both the numerator and denominator, yielding a result which can be measured directly:

$$
I_{s p}=\frac{I_{b i t}}{m_{\text {shot }} g}
$$


where $I_{b i t}$ is the impulse delivered by the thruster and $m_{\text {shot }}$ is the amount of mass ejected to deliver it. Similarly, the conventional thrust efficiency is given as:

$$
\eta_{t}=\frac{F^{2}}{(2 \dot{m} P)}
$$

where $\mathrm{P}$ is steady electric power supplied. When the idealistic assumption is made that the shape of the thrust over the duration in which it is delivered does not create a large discrepancy between the square of the integral of the thrust and the integral of the thrust squared, we find that the pulsed analog to this efficiency must become:

$$
\eta_{t}=\frac{I_{b i t}{ }^{2}}{\left(2 m_{\text {shot }} E_{o}\right)}
$$

where the variables are as previously defined. In addition, a more accurate subsystem level efficiency can be found by multiplying the thrust efficiency by the efficiency of the power conditioner.

\subsubsection{The PPT Dynamic Effects Model}

As a result of the highly complicated processes taking place at the interface of the Teflon propellant and the electric arc, the model developed and used herein for analysis does not endeavor to incorporate these physical phenomena. Instead, it focuses on the operational characteristics and limitations fundamental to the thruster system and attempts to provide reasonably representative figures to be used in evaluating their effects on the space system.

The most salient feature of the PPT is its pulsed operation. With a fixed thrust magnitude and a fixed pulse width, the thruster's ability to match and effectively null any forces causing departures from the spacecraft's desired position is limited to a dead-band scenario. A dead-band situation is the idealized process of counteracting a disturbance by periodic thrusting. Figure 4.2 shows a schematic of the position and velocity as a function of time, when the spacecraft is in a constant disturbance force environment. $x(t)$ is the spacecraft 


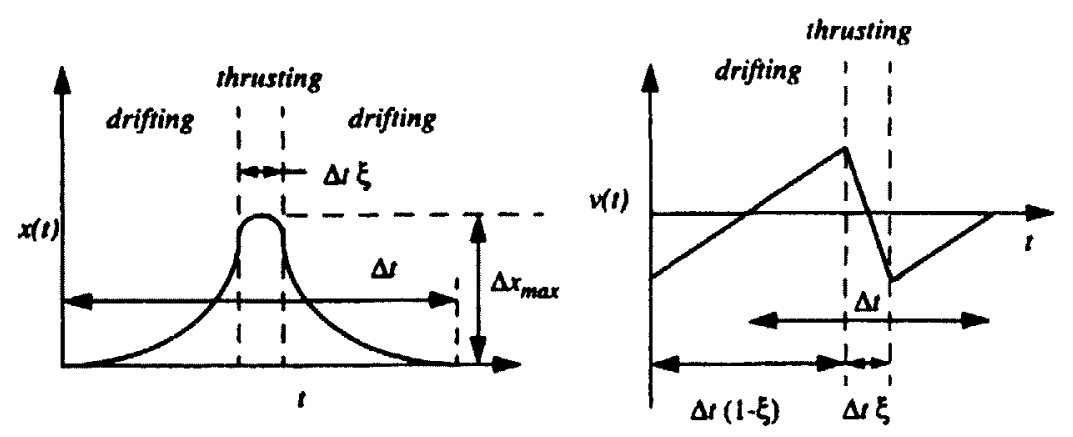

Figure 4.2 Idealized dead-band scenario schematic under a constant disturbance force [London, 1996] (adapted).

position as a function of time, $v(t)$ is the velocity as a function of time, $\Delta t$ is the time required to return to a specific position and $\xi$ is the fraction of the time $\Delta t$ during which corrective thrusting is performed. The minimum size dead-band is dependent on the pulse width, magnitude and pulse repetition frequency (PRF) in addition to the spacecraft mass properties and the external disturbance environment.

Using the various requirements and characteristics for each mission, the PPT model simulates the thruster's ability to maintain position in an environment with a constant or time varying disturbance force. Various thruster parameters can be adjusted to meet the performance specifications of the mission. These parameters include impulse bit, pulse width, maximum PRF and pulse height. They in turn determine important system metrics like average and peak power consumption, propellant usage and thruster size.

This model, as well as the other propulsion system models, was implemented by writing a time stepping simulation in Matlab ${ }^{\circledR}$. The dynamic properties of the propulsion system can be captured by a simple one dimensional model. The three dimensional effect can be handled without numerical simulation, if a fairly isotropic disturbance environment is assumed. For example, we may assume that in a worst case scenario the dead-band width specified is only maintained for each axis direction independently. In this case the largest migration from the desired position will be the square root of the sum of the squares of the dead-band distances in each direction. If it is assumed that the desired dead-bands would 
be equal in each direction, then the actual maximum distance from the desired position of the spacecraft would be $\sqrt{3} \Delta x$.

The simulation treats the spacecraft as a point mass and has the capability of subjecting it to a constant or time varying disturbance force. Using conditional logic (with position and velocity as inputs) and the constraints inherent in the thruster characteristics, a thrust signal is created which keeps the spacecraft within the pre-specified dead-band. The decision of whether to pulse or not is made each time step. In order to make this decision The net impulse on the spacecraft (divided by the mass to give velocity) is integrated to determine the spacecraft's distance from its desired position. If that position (or error in position) is outside of the effective dead-band (which can be tuned somewhat to prevent overshoot), and the spacecraft velocity is directed away from the desired position, and it has been at least as long since the last pulse as the specified time required to recharge the capacitor, the thruster is fired. The direction, or sign, of the thrusting is determined by the sign of the position error. Depending on the size of the impulse bit, opposing thrusters may need to be fired to keep the spacecraft in the dead-band. In general this condition suggests that the thrusters are oversized for the disturbance force they are fighting and the dead-band they are trying to maintain. As a result of the one-dimensionality of the simulation, it models the firing of two thrusters, one in the positive and one in the negative direction.

The time scale of the PPT pulse width is extremely short compared to the duration of the simulation. Therefore, it is impractical to simulate the thrust signal itself by time marching. Instead an impulse profile is created by time stepping and the thrust signal is derived from it by differentiating afterward. In doing this, one might expect a numerical singularity to occur when the derivative of a step function is taken. However, the simulation requires a single time step to change the value of the impulse. By requiring this time step to be the width of a single pulse, the differentiation produces spikes of the correct height and width (so that they integrate to the value of the specified impulse bit). An example plot of the cumulative disturbance and thruster impulses is shown in Figure 4.3. 


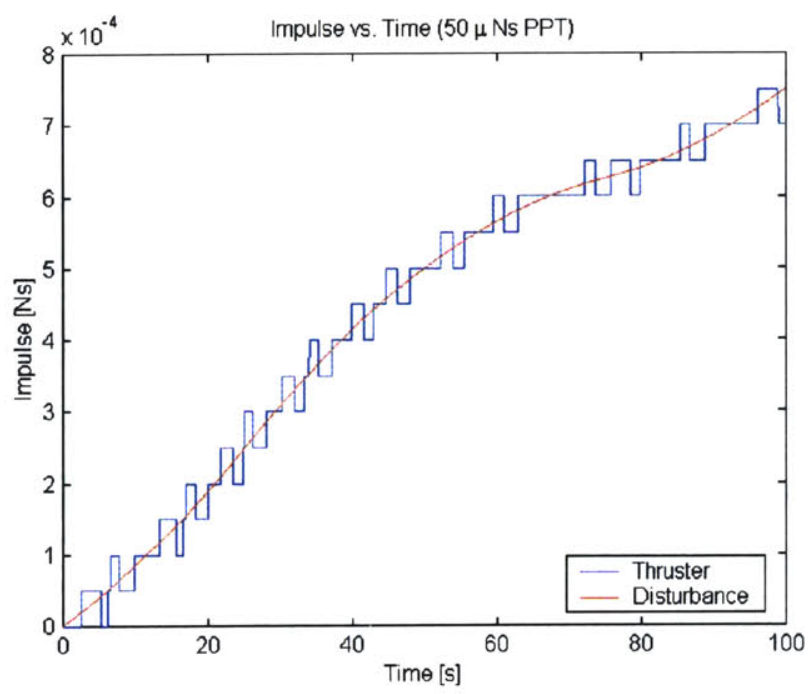

Figure 4.3 PPT cumulative impulse plot showing disturbance in red and thruster actuation in blue.

The precision to which the thruster can follow and cancel the disturbance is constrained by the minimum impulse applied by the thruster and the maximum PRF it can support. The ranges that these parameters can vary through are constrained by the thruster physics. In general, based on data from current technology and experimental work, the PRF can be no more than $6 \mathrm{~Hz}$ or so, while the impulse bit is unlikely to be any less that about $20 \mu \mathrm{N}-\mathrm{s}$.

There is work underway to create a $2 \mu \mathrm{N}$-s PPT, but only very limited information is currently available about their performance including an impulse bit to impulse bit variability of $\pm 74 \%$, which could present a large problem for precision control applications [Mueller, 2000]. For the following simulation examples the parameters used are as shown in Table 4.2.

TABLE 4.2 Summary of example simulation parameters

\begin{tabular}{|c|c|c|c|c|c|c|}
\hline & $\begin{array}{c}\text { Avg. } \\
\text { disturbance }\end{array}$ & $\begin{array}{c}\text { Impulse } \\
\text { bit }\end{array}$ & $\begin{array}{c}\text { Max. } \\
\text { PRF }\end{array}$ & $\begin{array}{c}\text { Dead- } \\
\text { band }\end{array}$ & $\begin{array}{c}\text { Simulation } \\
\text { time }\end{array}$ & $\begin{array}{c}\text { S/C } \\
\text { mass }\end{array}$ \\
\hline \hline Value & $7.5 \mu \mathrm{N}$ & $50 \mu \mathrm{Ns}$ & $4 \mathrm{~Hz}$ & $0.25 \mu \mathrm{m}$ & $100 \mathrm{~s}$ & $200 \mathrm{~kg}$ \\
\hline
\end{tabular}


The tunable parameters in the PPT model include the disturbance profile and magnitude, the impulse bit, the maximum PRF, the dead-band distance requirement and the simulation duration as well as the spacecraft mass. For the example figures from the simulation presented in this chapter the disturbance force on the spacecraft has a saw-tooth profile with an average of $7.5 \mu \mathrm{N}$, a maximum of $12.5 \mu \mathrm{N}$, a minimum of $2.5 \mu \mathrm{N}$ and a period of approximately 100 seconds. Figure 4.4 shows a plot of the disturbance force versus time.

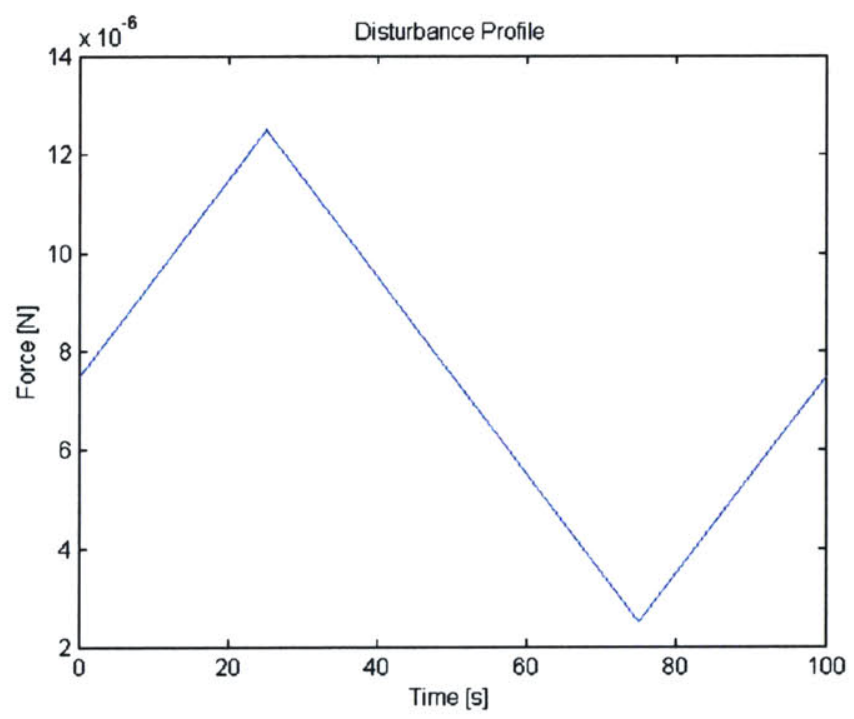

Figure 4.4 Disturbance force used for the example simulation runs.

From the impulse plot, the thrust signal is derived by simply differentiating the net impulse imposed on the spacecraft. Using the same parameters as the example shown in Figure 4.3, the thrust profile shown in Figure 4.5 is generated. It may be noted that thrusting in both directions is required in this case, suggesting an oversized impulse bit given the very small dead-band $(0.25 \mu \mathrm{m})$. This effect can be seen in the trajectory shown in Figure 4.8 , which shows the spacecraft position crossing the dead-band very quickly after a thruster pulse and requiring another thrust in the opposite direction.

Figure 4.6 shows an example of the power spectral density of the net acceleration on a spacecraft using PPTs to cancel a disturbance force. The same simulation was used to 


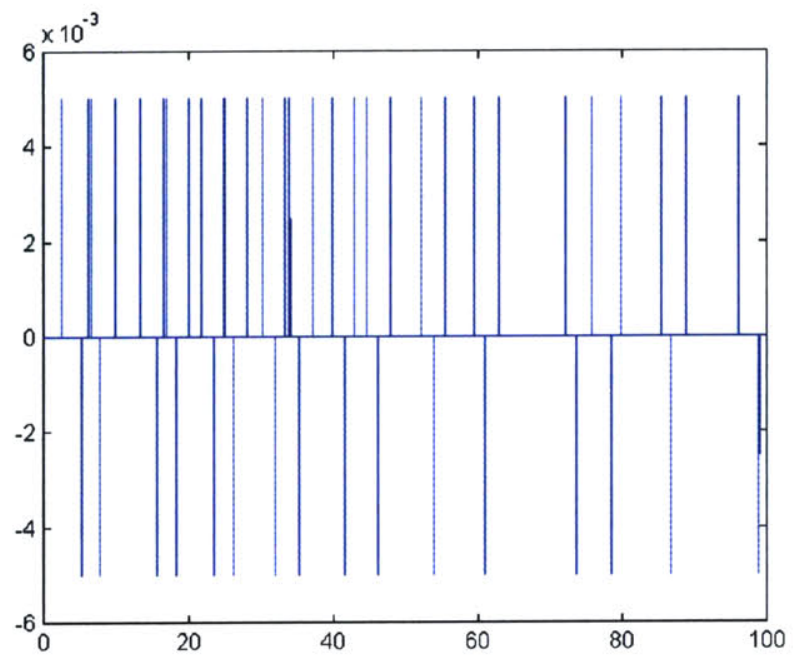

Figure 4.5 PPT thrusting profile for position maintenance, exemplified.

generate Figure 4.6 as was used for Figure 4.3 and Figure 4.5. Additionally, a power

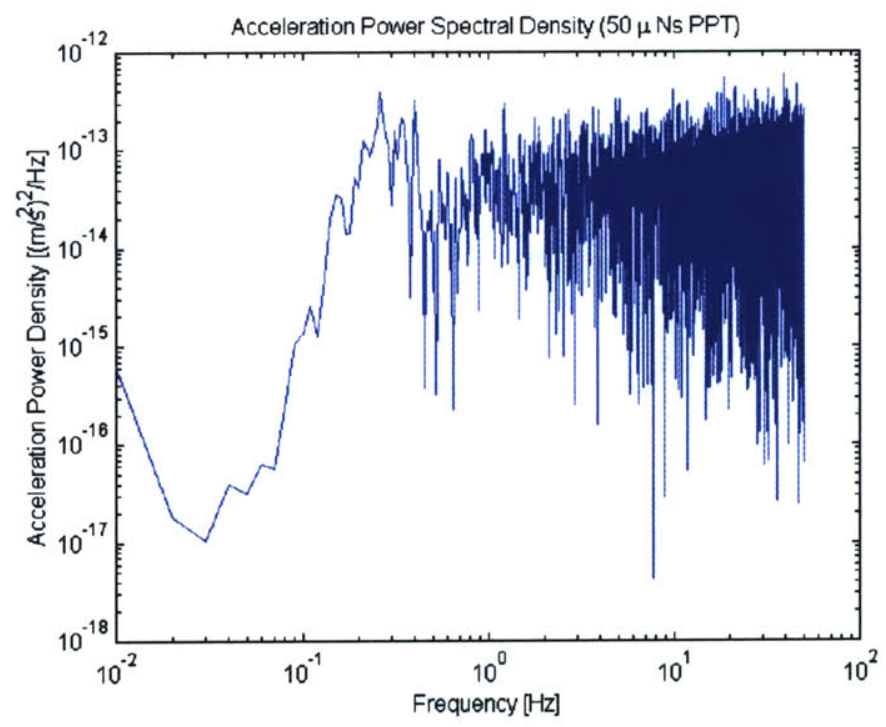

Figure 4.6 Net acceleration power spectral density (PSD) example.

spectral density (PSD) of the spacecraft position can be computed and is shown in 
Figure 4.7. The PSD is a representation of the frequency content of a time signal. Its

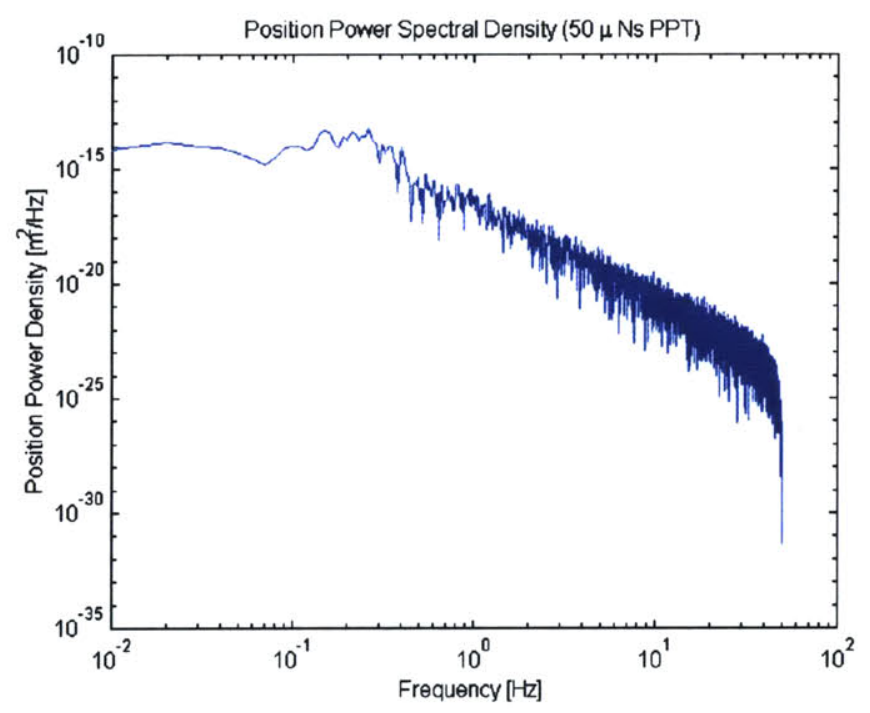

Figure 4.7 Position power spectral density example (from trajectory).

magnitude can be approximated by taking the square of the Fourier transform of the time signal. A more detailed explanation of the PSD is presented in the previous chapter in Section 3.3.1. In general, this type of analysis is rare when evaluating propulsion systems. However, in instances when the spacecraft is especially sensitive to specific types of noise, that is, noise within a certain frequency band, this analysis often seems more illuminating than some more conventional techniques.

Further characteristics of the propulsion system can be derived from the simulation. By integrating the total impulse experienced by the spacecraft, the position vs. time plot or trajectory of the spacecraft with respect to a desired position can be computed. An example of such a trajectory is shown in Figure 4.8, where the red horizontal lines demark the dead-band limits imposed on the spacecraft at the beginning of the simulation. If a deadband requirement is specified in the mission requirements, this plot can be used to verify that the specified thruster size (impulse bit) supplies the appropriate performance. By iterating this process, the most efficient size PPT can be chosen for a given position mainte- 


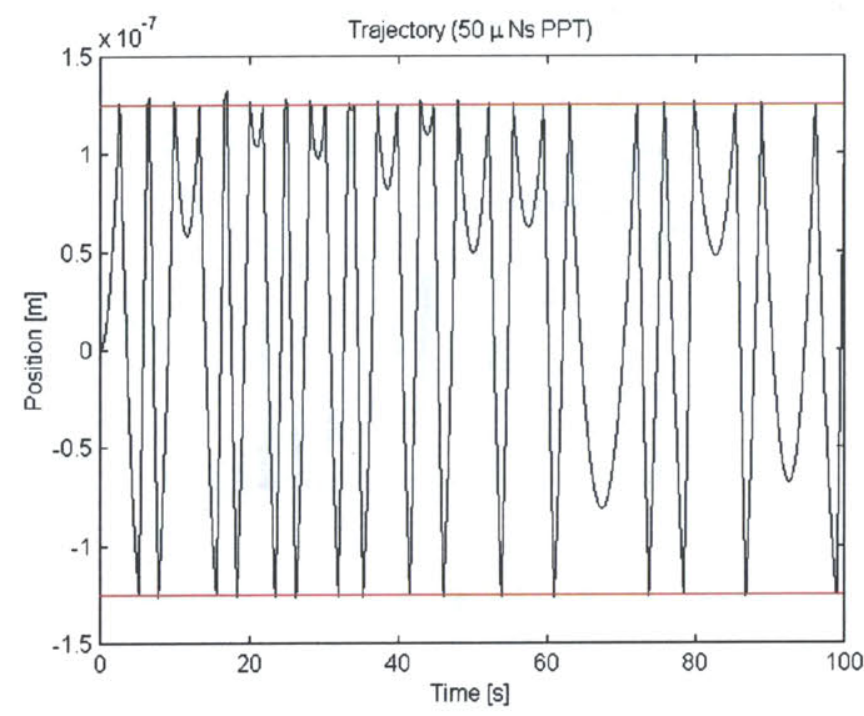

Figure 4.8 One dimensional spacecraft trajectory about a desired position.

nance accuracy requirement. This process was not carried out rigorously in this study. However, a few iterations were performed for each of the designs (presented in Chapter 5) to eliminate excessive firing on both sides of the dead-band.

\subsubsection{General Attributes and Trends}

The stability characteristics of the model should also be noted. In order for the model to maintain spacecraft position stability, the impulse bit must be properly sized for the disturbance environment and the required dead-band size. Because impulse bits cannot yet generally get smaller than about $20 \mu \mathrm{Ns}$ and pulse frequencies can be no larger than about 6 $\mathrm{Hz}$, a PPT which can maintain a small dead-band width will not be able to perform functions requiring higher thrust values (normally expected to be attainable by increasing pulse frequency). However, there may still be a range of acceptable impulse bits for a given dead-band maintenance criterion.

A simple non-dimensional analysis of the situation provides some insight. Assuming a case with a constant disturbance force and therefore, periodic thrusting, the parameters 
involved in the situation include: impulse bit $\left(\mathrm{I}_{\mathrm{bit}}\right)$, disturbance force $\left(\mathrm{F}_{\mathrm{D}}\right)$, spacecraft mass $(\mathrm{m})$, dead-band width $(\Delta \mathrm{x})$ and frequency $(f)$. From these five variables we know that two non-dimensional groups can be formed. They are

$$
\frac{l_{b i f} f}{F_{D}} \text { and } \quad \frac{F_{D}}{m \Delta x f^{2}}
$$

From the physical situation that has been constructed we can say:

$$
\begin{aligned}
& \frac{l_{b i f} f}{F_{D}} \sim 1 \quad \text { and } \quad \frac{F_{D}}{m \Delta x f^{2}} \leq 2 \\
& \text { So, } \quad \frac{I_{b i f} f}{m \Delta x f^{2}} \leq 2 .
\end{aligned}
$$

The first expression in Equation 4.8 comes from the need to balance the disturbance impulse with the impulse imparted by the thruster, while the second expression is a translation of the kinematics into the parameters involved in the dead-band situation.

With a larger impulse bit, fewer pulses will be required. First, this will impart disturbances to the spacecraft in slightly lower frequency bands, whereas smaller impulse bits will be required more frequently and therefore show up more predominantly in the high frequency range. Perhaps more importantly, using larger impulse bits may end up leading to more fuel usage because of the inability to accurately match the disturbance induced $\Delta v$ without pulsing in opposing directions. However, larger PPTs do tend to operate slightly more efficiently, potentially canceling these effects.

\subsection{Colloid Thruster}

\subsubsection{Background \& Fundamentals}

Much like the PPT, the colloid thruster was first proposed in about 1960 and research continued intensively until about 1975 when several factors caused a lack of interest and funding for colloid thruster research. One of the key factors was the desire for higher 
thrust, which would require very high voltages in the range of 12 to $100 \mathrm{kV}$ and thus cause packaging problems [Martinez-Sanchez, 1999b]. Recently, with the need for small, precise thrusters for microsatellite missions, colloid thrusters are being investigated again. In contrast to the PPT which was flown early in its 'life', the colloid thruster has never flown on a space mission.

The basic operation of a colloid thruster involves extracting charged droplets (and/or lone ions) from an electrolytic liquid using strong electric fields. Common examples of propellant mixtures include combinations of formamide or glycerol as solvents and sodium iodide (NaI) or lithium chloride $(\mathrm{LiCl})$ as solutes. Other liquid and salt combinations are possible. One class of very new and particularly attractive options lies in ionic liquids, which are composed completely of ions, require no solvent and are very highly conductive. Figure 4.9 shows a schematic of a single needle colloid emitter's main elements.

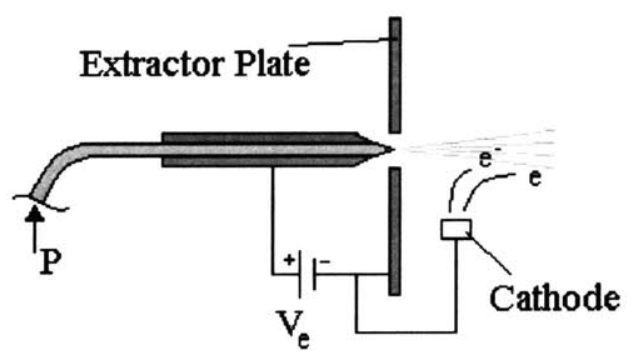

Figure 4.9 Single-needle colloid thruster schematic.

The lightest grey shading represents the propellant, while the annular extracting plate and conducting needle are shown in a darker grey. A power supply is used to establish a voltage difference between the extractor and needle creating an electrostatic traction force on the surface of the fluid meniscus that forms. This force, balanced with the fluid surface tension and possible back pressure on the fluid results in the formation of a cone that emits a jet of droplets at its vertex. Then, these droplets are accelerated through the potential to a high speed. The liquid cone has a vertex half angle, $\alpha=49.30$, regardless of the fluid 
properties, and is called a Taylor cone in honor of its discoverer [Taylor, 1964]. In order to cause the instability in the fluid meniscus and create a Taylor cone, the linear instability condition,

$$
E>\sqrt{\frac{\gamma_{\mathrm{st}} k}{\varepsilon_{o}}},
$$

must be met [Zeleny, 1917], where $E$ is electric field strength, $\gamma_{\mathrm{st}}$ is fluid surface tension, $\mathrm{k}$ is the surface wave number and $\varepsilon_{\mathrm{o}}$ is the permittivity of free space. Once a cone-jet is formed, there are many regimes of operation in which it may lie. This is determined primarily by the propellant and the electric field. In what is called the stable single cone-jet regime, the behavior of the current can be characterized by

$$
I=f(\varepsilon)\left(\frac{\gamma_{\mathrm{st}} \kappa Q}{\varepsilon}\right)^{\frac{1}{2}}
$$

where $\varepsilon$ is the dielectric constant of the propellant, $\gamma_{\mathrm{st}}$ its surface tension, $\kappa$ the conductivity, Q the volumetric flow rate and $f(\varepsilon)$ is a function which is between 18-25 when $\varepsilon$ is larger than 40 and drops linearly below that value for smaller values of epsilon [Fernandez de la Mora, 1994]. The non-dimensionalized volumetric flow rate parameter gives bounds for the stable single cone-jet operation regime. This parameter:

$$
\eta=\left(\frac{\rho \kappa Q}{\gamma_{\mathrm{st}} \varepsilon \varepsilon_{o}}\right)^{\frac{1}{2}}
$$

where $\rho$ is fluid mass density and the other parameters are as previously defined, should be no less than approximately one (where the minimum operating flow rate is found) and no greater than some value between three and ten.

The thrust can be written as,

$$
F=\dot{m} v=\rho Q \sqrt{2 \mathrm{~V}(q / m)}
$$


which is derived from the standard thrust equation, where $\mathrm{V}$ is the voltage through which a droplet is accelerated and $(\mathrm{q} / \mathrm{m})$ is the charge to mass ratio of an emitted droplet. Substituting $(\mathrm{I} / \dot{m})$ for this ratio gives a definition for thrust composed of only known or measurable parameters:

$$
F=\sqrt{2 f(\varepsilon) \rho Q}\left(\frac{\gamma_{\mathrm{st}} \kappa Q}{\varepsilon}\right)^{\frac{1}{4}}
$$

As can be seen from equation 4.13 , the thrust scales as the volume (or mass) flow rate to the $3 / 4$ power. Accordingly, the specific impulse scales as $\dot{m}^{-1 / 4}$, since it is simply thrust divided by mass flow rate and the gravitational constant, g. Therefore, the specific impulse for this electric thruster is the same gravitational constant normalized exhaust velocity that is conventionally used:

$$
I_{s p}=\frac{\mathrm{v}}{g}=\frac{\sqrt{2 \mathrm{~V}(q / m)}}{g} .
$$

While the efficiency of the colloid thruster system is relatively high, there are a few loss mechanisms that degrade the performance. These losses include the impingement of droplets on the extractor electrode, a voltage drop on the order of 100 volts required to form the droplets and the degree of polydispersity of the spray. The first effect is expected to be negligibly small, the second amounts to a loss in efficiency of between two and four percent and the third effect can be significant, in particular, when the thruster is operated in a regime where a sizable fraction of the current emitted is due to single ions. The propulsive efficiency due to polydispersity effects is formulated as:

$$
\eta_{t}=\frac{F^{2}}{2 \dot{m} I \mathrm{~V}}=\frac{\left(\sum_{j} \dot{N}_{j} \sqrt{2 m_{j} q_{j} \mathrm{~V}}\right)^{2}}{2 \mathrm{~V}\left(\sum_{j} \dot{N}_{j} m_{j}\right)\left(\sum_{j} \dot{N}_{j} q_{j}\right)},
$$


where the normal performance parameters in the efficiency equation are written in terms of the sums of the number of particles per second, $\dot{N}_{j}$, with a given property, charge $\left(q_{j}\right)$ or mass $\left(m_{j}\right)$, as shown here:

$$
\begin{aligned}
& \dot{m}=\sum_{j} \dot{N}_{j} m_{j} \quad I=\sum_{j} \dot{N}_{j} q_{j} \\
& F=\sum_{j} \dot{N}_{j} m_{j} c_{j}=\sum_{j} \dot{N}_{j} \sqrt{2 m_{j} q_{j} \mathrm{~V}} \quad c_{j}=\sqrt{\frac{2 q_{j} \mathrm{~V}}{m_{j}}}
\end{aligned}
$$

When it is assumed that there are just two types of particles, droplets (which we take to be monodisperse by themselves) and ions, we can define each of the quantities in Equation 4.16 as the sum of just two j's. For example, the current is written as:

$$
I=\left(\dot{N}_{i} q_{i}+\dot{N}_{d} q_{d}\right)
$$

where ' $i$ ' denotes ions and ' $d$ ' denotes droplets. Making these substitutions in the original equation (Equation 4.15) and defining two variables, which are ratios of other variables as indicated in Equation 4.18, we find an expression for the thrust efficiency due to the polydispersity effect:

$$
\begin{aligned}
& \eta_{t}=\frac{\left[1-(1-\sqrt{\phi}) \beta_{i}\right]^{2}}{1-(1-\phi) \beta_{i}}, \\
& \beta_{i}=\frac{I_{i}}{I} \quad \phi=\frac{(q / m)_{d}}{(q / m)_{i}}
\end{aligned}
$$

where $(q / m)_{d}$ is the charge to mass ratio of the droplets emitted, $(q / m)_{i}$ is the charge to mass ratio of the ions emitted, $I_{i}$ is the current carried by the ions and $I$ is the total current emitted, comprised of the current contributed by both the ions and the droplets. From this equation we can find that the minimum efficiency lies at a value of

$$
\eta_{t}=\frac{4 \sqrt{\phi}}{(1+\sqrt{\phi})^{2}}
$$


indicating that the two regimes of high efficiency are (as would be expected) when the propellant is exhausted as either almost all ions or almost all droplets.

\subsubsection{The Colloid Thruster Control and Dynamic Effects Model}

The colloid thruster model assumes an array configuration. That is, a large number of individual needles emitting charged droplets at a nominal operating point are arranged so that the thrust of the entire array may be throttled over a range. The physical arrangement considered from here on assumes that blocks, or groups, of needles (several of which make up an entire 'thruster') can be turned on or off independently from other blocks and the overall accelerating voltage of the thruster can be varied, while the extracting voltage is always either on at the nominal value or zero. A schematic illustration of this concept is presented in Figure 4.10, and should clarify the setup described above. The grey plate is

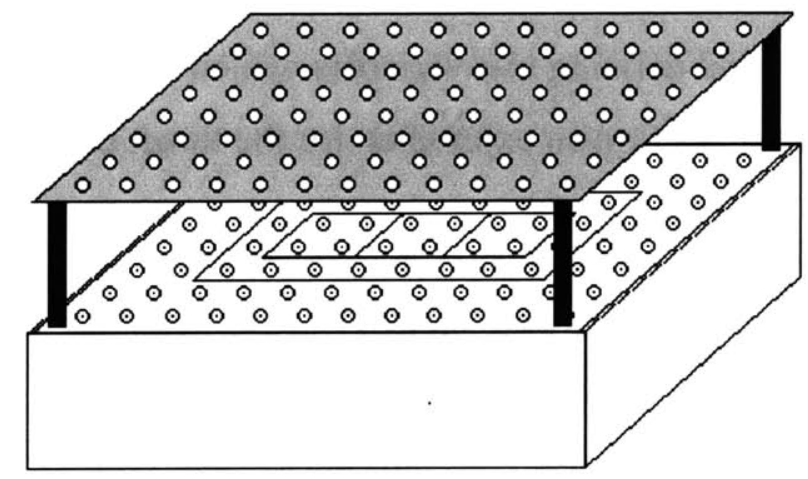

Figure 4.10 Colloid thruster array schematic.

the accelerator, which stands off from the plane of the extractor (shown in white). The length of the black stilts upon which the accelerator rests are greatly exaggerated, just to show the configuration of the blocks below it. Each of the circles on the lower, white plane is a hole in the extractor, in which a needle sits. Groups of these circles are separated from each other into units previously referred to as blocks. Each of these blocks can have its extractor turned on (raised to some nominal potential) independently of the other 
blocks. Unless the thruster is completely turned off, the accelerator plate sits somewhere between the voltage of the extractor and its own maximum voltage. The accelerator plate is sufficiently far from the needles that if there is no potential bias on a needle's extractor, no emission will occur as a result of the accelerator voltage. However, if the extractor is 'turned on' for at least one of the blocks, the accelerator will increase the kinetic energy of the emitted droplets by providing a larger potential down which they fall.

As a result, the thruster unit has two levels of thrust control. The coarser level determines how many needles are emitting and the finer level determines the depth of the potential the droplets are accelerated through. The system can therefore be designed in such a way as to create continuous throttling capability through a range of thrusts far exceeding that of a single needle, if an appropriate controller is used.

The actual number of blocks and needles per block used for the simulations in this study is determined by a combination of the maximum thrust required, an assumed maximum voltage for the accelerator and the fineness of thrust increments desired, which is based on the formation flying requirements of the particular missions. The total number of needles is determined by Equation C.16 and is equal to the ratio of the maximum desired thrust to the thrust per needle multiplied by the square root of the ratio of the nominal voltage to the maximum voltage. A value of 5 kilovolts was adopted for the maximum voltage in all the simulations. The thrust per needle required is determined by all of the fundamental physics of the thruster. Two different needle designs were put together. One for relatively strict mission requirements $\left(\mathrm{F}_{\mathrm{N}}=.13 \mu \mathrm{N}\right)$ and the other for somewhat more lenient ones $\left(\mathrm{F}_{\mathrm{N}}=.5 \mu \mathrm{N}\right)$. One of these two designs was used for each of the missions discussed. Then, the number of needles in each block and the number of blocks was just built up by to allow thrusting over the full range required by the mission without needing an acceleration voltage of greater than 5 kilovolts. For example, Table 4.3 shows the thrust and the number of needles per block for the first several blocks in the colloid thruster design for the SPECS mission. 
TABLE 4.3 Block and needle increments for the SPECS colloid thruster design.

\begin{tabular}{|c|cccccc|}
\hline & Block 1 & Block 2 & Block 3 & Block 4 & Block 5 & Block 6 \\
\hline \hline Needles [ ] & 1 & 2 & 5 & 13 & 33 & 85 \\
Thrust $[\mu \mathrm{N}]$ & 0.13 & 0.26 & 0.65 & 1.69 & 4.29 & 11.05 \\
\hline
\end{tabular}

The model described herein captures these aspects of 'controlling' the thruster in addition to representing some physical characteristics of the colloid thruster operation, albeit in a crude way. The most obvious of these characteristics is the fact that when a particular block of the thruster is commanded to begin emitting there will be some lag time required for the fluid menisci on each needle in the block to form into cone-jets and emit droplets. In order to model this, we consider the simplest scenario, which is illustrated in Figure 4.11. Before, the extractor electrode is activated the fluid at the tip of the needle
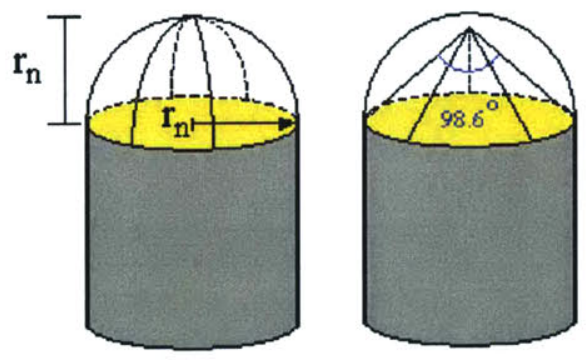

Figure 4.11 Illustration of change in fluid meniscus at needle tip necessary before colloid emission can begin.

will be in the form of a hemispherical meniscus with radius equal to the inner radius of the needle, if the pressure at the end of the needle is balance exactly by the surface tension. When in steady state mode the fluid at the tip of the needle must be in the form of a Taylor cone with a tip half-angle of $49.3^{\circ}$. Therefore, the volume of fluid that needs to be displaced to change between the two shapes divided by the flow rate of the fluid gives a first order approximation of the delay between the time when the extractor electrode is activated and when the needle can begin emitting. The calculation is simply: 


$$
\begin{aligned}
& V_{\text {hemisphere }}=\frac{1}{2}\left(\frac{4}{3} \pi r_{n}^{3}\right) \\
& V_{\text {cone }}=\frac{\pi r_{N}^{3}}{3 \tan \alpha} \\
& t=\frac{V_{\text {hemisphere }}-V_{\text {cone }}}{Q}=\frac{2}{3} \frac{\pi r_{n}^{3}}{Q}\left(1-\frac{1}{2 \tan \alpha_{T}}\right)
\end{aligned}
$$

where $r_{n}$ is the inner radius of the needle, $\alpha_{T}$ is the cone vertex half-angle, $V$ is volume, $t$ is the time delay and $Q$ is the volumetric flow rate. This time delay is very sensitive to the needle radius, so estimates can range from 50 milliseconds or more down to less than 3 milliseconds. Not only does this scenario lead to a time delay for emission, but likely causes an overshoot in the current and thrust because of the ejection of a relatively large droplet of propellant.

However, if the needle and feed system can be designed such that the back pressure force at the needle tip is less than the surface tension, this delay may be reduced to a negligible time and the emission of the large droplet eliminated. In effect, electrostatic forces are doing more work in extracting the fluid, initially, than previously when more help was provided by the tank-supplied back pressure. In order to eliminate the displacement of a large drop of liquid at the start of emission, when no electric field is applied, the meniscus of fluid must have the same volume as the Taylor cone that will be created during thrusting and it will need to be less than a full hemisphere. By geometric arguments, the pressure required for this scenario should be approximately

$$
p_{m}=\frac{2 \gamma}{r_{N}} \sqrt{1-\left(1-\frac{1}{2 \tan \alpha_{T}}\right)^{2}} \text {. }
$$

For a needle of inner radius equal to 20 microns and a surface tension similar to that of formamide or glycerol this pressure is approximately $6000 \mathrm{~Pa}(60 \mathrm{mbar})$. If this scenario can be implemented, the delay is estimated to be within a couple of orders of magnitude of one hundred nanoseconds, which is negligible. In the modeling and design, however, the 
worst case scenario presented previously will be adopted in order to be conservative. One additional non-ideality that is not modeled is the caused by the fact that a rounded meniscus requires an initial instability-creating voltage that is greater than the voltage necessary for the maintenance of a stable Taylor cone-jet. This effect is likely due to an intensification of the electric field caused by the reduction in the characteristic size of the cone jet as compared to that of the bulbous, hemispherical type of meniscus. So, when the extractor for a block of needles is turned on, it must be brought up to the starting voltage and then reduced to the nominal operating voltage. The additional voltage needed for emission initiation is estimated to be approximately two or three hundred volts, which is typically about ten percent of the starting voltage. A simple way to do this is by inserting a 'ballast' resistor in series with the thruster. Initially, when voltage is applied there is no current, so the full applied voltage is seen by the propellant. Once current begins to flow, the voltage drop due to the ballast, $I R_{\text {ballast }}$, can be selected to reduce the steady state voltage to the desired level. This technique incurs some inefficiency, but it is minor.

The simulation for the colloid thruster produces the same kinds of results, in the form of plots, as that for the PPT. However, the colloid thruster model only requires one thruster because of the continuous nature of the operation and the very small thrust levels it provides, which are able to cancel the disturbance forces almost exactly. The control is designed to nullify the impulse imparted to the spacecraft by the disturbance force and to keep the spacecraft at the specified position. A disturbance profile is specified and it, coupled with logic to control the switching of the various blocks and the accelerator voltage, is used to produce a thrust profile, a trajectory and PSDs of both position and acceleration.

The following simulation results model the behavior for an array that consists of 77 needles arranged in five different blocks. Each needle provides a thrust of $0.2 \mu \mathrm{N}$ when operated at the nominal extracting voltage with no further acceleration potential. At the nominal operating voltage of 2500 volts the 5 blocks deliver a thrust of $0.4,1,2,4$ and 8 $\mu \mathrm{N}$. The maximum voltage on the accelerator grid is 5000 volts, bringing the maximum thrust of the array with all needles emitting to $21.8 \mu \mathrm{N}$. The example results shown in this 
section use the same disturbance force as that in the PPT example of Section 4.1.2. The average force is $7.5 \mu \mathrm{N}$ while the maximum and minimum forces are $12.5 \mu \mathrm{N}$ and $2.5 \mu \mathrm{N}$, respectively. Figure 4.12 shows the disturbance force plotted, in red, along with the thruster profile, in dashed black. As can be seen, the colloid thruster array has the ability

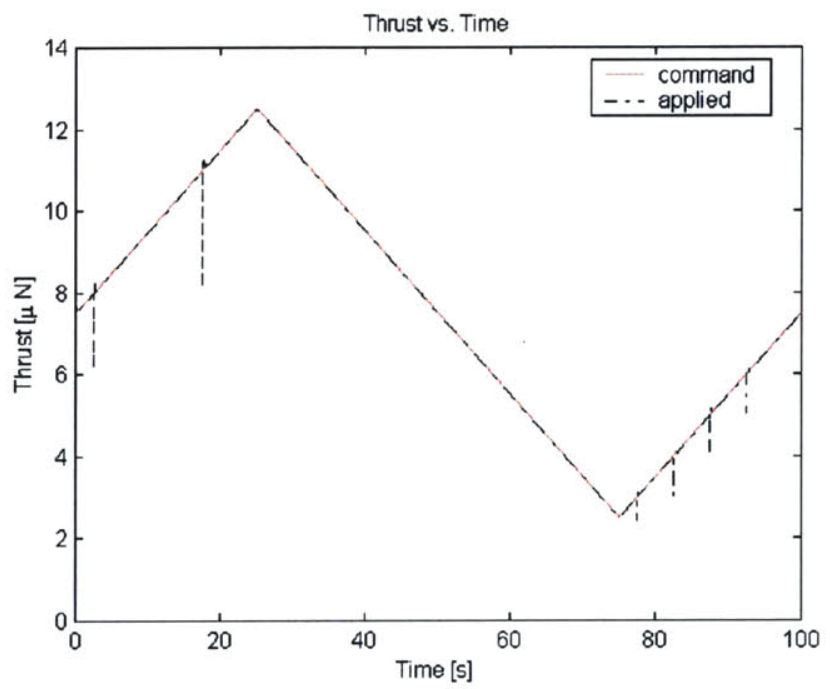

Figure 4.12 Colloid thruster thrust profile plotted with

to follow and cancel the disturbance force very closely except for the few blips that occur when the thrust level requires the starting of a new block. These lapses in thrust are about 0.1 seconds long in this run of the simulation, but they can be changed depending on the operating point decided on for the thruster. In order to achieve the thrust signal show in Figure 4.12 the accelerator electrode's voltage must be varied to scale the thrust emitted by the blocks of needles that are turned on. Figure 4.13 shows a plot of voltage versus time for the example simulation. The voltage remains between the 2500 volts of the extractor and the 5000 volt limit placed on the thruster design.

Based on these parameters and a 200 kilogram spacecraft the trajectory computed by the simulation is shown in Figure 4.14. It shows that the largest excursions of the spacecraft from the desired position are no more than a tenth of a nanometer. This small deviation 


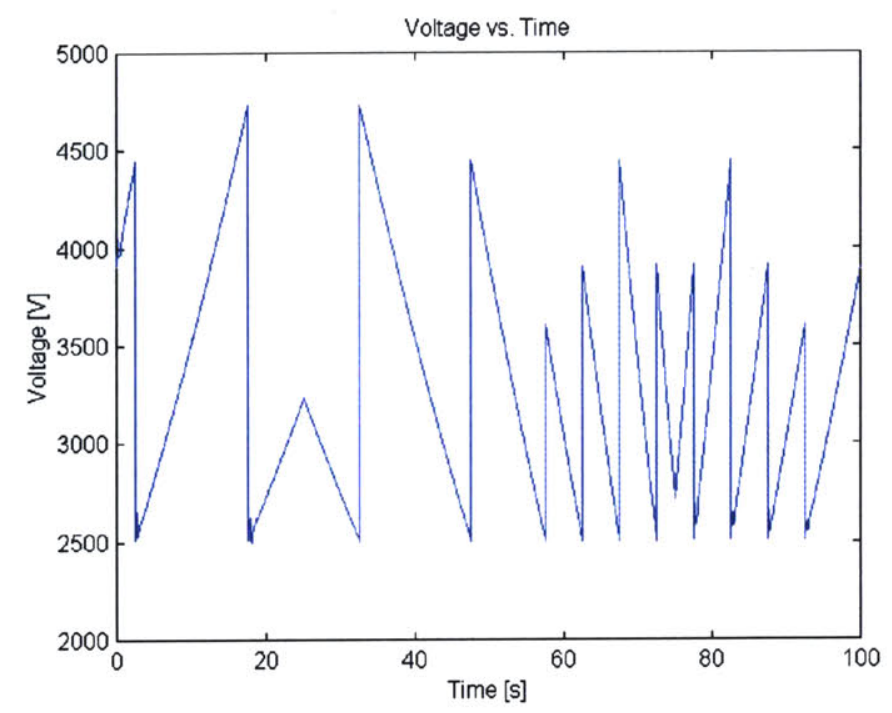

Figure 4.13 Colloid thruster array accelerator voltage for the example simulation.

from the desired position means that only minute amounts of acceleration and position power are expended. This translates into small magnitudes for position and acceleration PSDs, as shown in Figure 4.15 and Figure 4.16. These PSDs are the combination of spec-

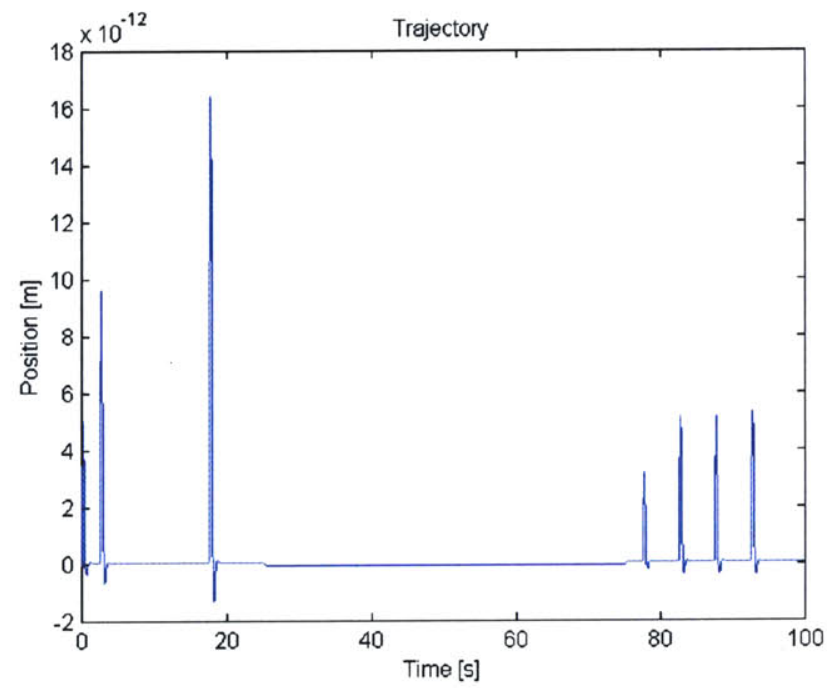

Figure 4.14 Spacecraft trajectory with colloid thruster maintaining position. 


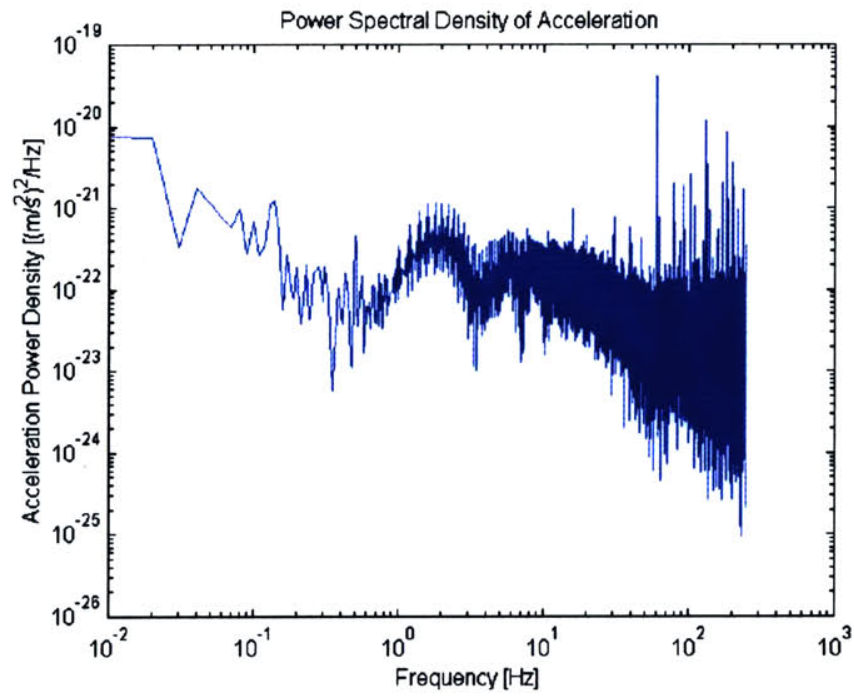

Figure 4.15 Net acceleration PSD for spacecraft with colloid thruster firing.

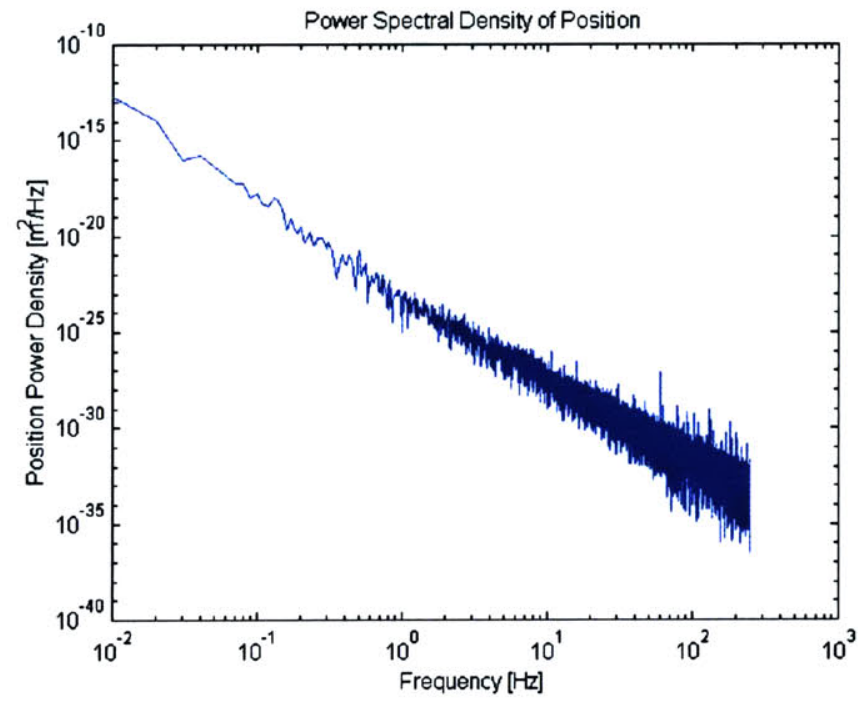

Figure 4.16 Position PSD for spacecraft with colloid thruster firing.

tral information from the simulation resulting from the control modeling and a steady state noise spectra provided by Busek Company, Inc. from single needle colloid thruster experimentation [Gamero, 2000]. It is seen that most of the power is at relatively low frequencies. This results from the facts that the higher frequency fluctuations, due to current 
instability, are very small in magnitude and the spacecraft not only makes nothing but small deviations from its desired position, it also only makes these excursions relatively infrequently.

\subsubsection{General Attributes and Trends}

One of the attributes that makes the colloid thruster array a potential option for varied mission types is the ability for the thrust range to be suited perfectly to a given mission without affecting the efficiency of operation (electrical efficiency and specific impulse). This is achieved by changing the total number of needles, the number of blocks and the number of needles within the blocks without affecting the performance achieved by each needle. In addition, the nominal operating point of each of the needles can be designed to meet the minimum thrust increment requirements that may be levied by a particular mission.

\subsection{Field Emission Electric Propulsion Thruster (FEEP)}

\subsubsection{Background \& Fundamentals}

The field emission electric propulsion device operates in a manner similar to the colloid thruster in that it directly extracts charged particles from a liquid propellant. The main differences lie in the propellant used and the voltage operating regime. Instead of using an electrolytic fluid, the FEEP uses a liquid phase metal, like cesium or indium, which are particularly attractive because of their low ionization potential, high atomic weight and low melting point. Ions are extracted directly by field emission and subsequently accelerated down an electric potential. This requires FEEP thrusters to be operated at higher voltages than colloids in order to overcome the ionization potential. Colloid thrusters can also emit a combination of droplets containing ions and individual ions, allowing operation at lower voltages.

A typical cesium FEEP thruster is shown in schematic in Figure 4.17. It consists of a slit shaped emitter, in which a propellant reservoir is located. Typical slit dimensions are 1 or 


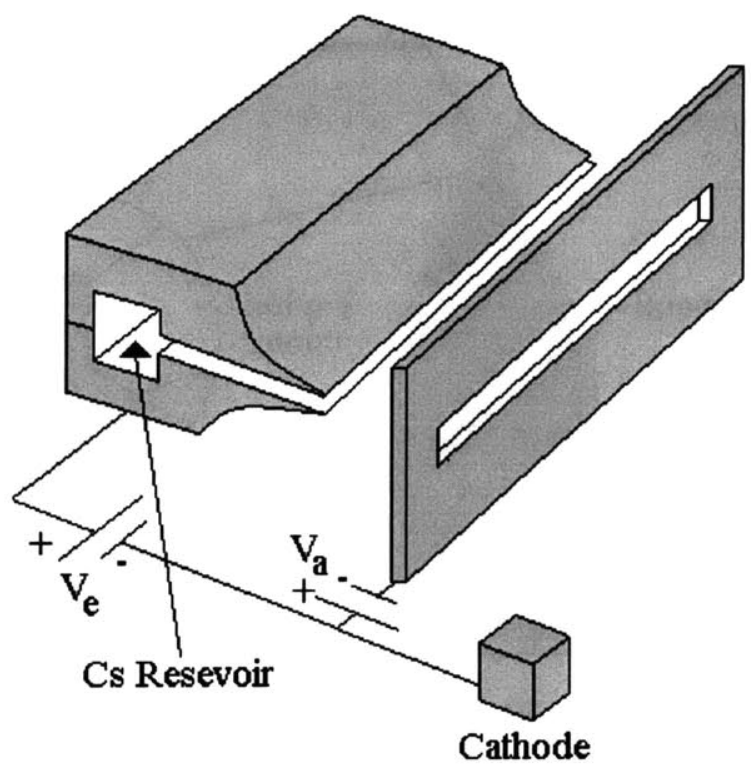

Figure 4.17 FEEP thruster schematic illustration (not to scale).

2 microns in height and anywhere between 1 millimeter to several centimeters in length. The extractor plate is biased at a negative potential of several kilovolts. The distance between the emitter and the extractor is greatly exaggerated for clarity. A neutralizer is also necessary since the beam consists only of positive ions.

Another version of the FEEP thruster, which has been under development at the Austrian Research Centers for several years, uses liquid indium as a propellant. It can be understood as a cross between the cesium FEEP and the colloid thrusters. A solid needle and an extractor plate are biased (the extractor negative) and the potential between them allows a thin film of liquid indium from a pool at the base of the needle to be drawn up around the outside of the needle, coating it. A Taylor cone is formed at the tip of the needle as a result of the application of the appropriate voltage and a jet emits individual ions forming a beam. A schematic diagram of the indium FEEP is shown in Figure 4.18. Though, not shown in the diagram, a neutralizer is required in this case as well. These indium FEEP thrusters have been laboratory tested and flown (though as spacecraft potential controllers) and data is available on their noise characteristics. 


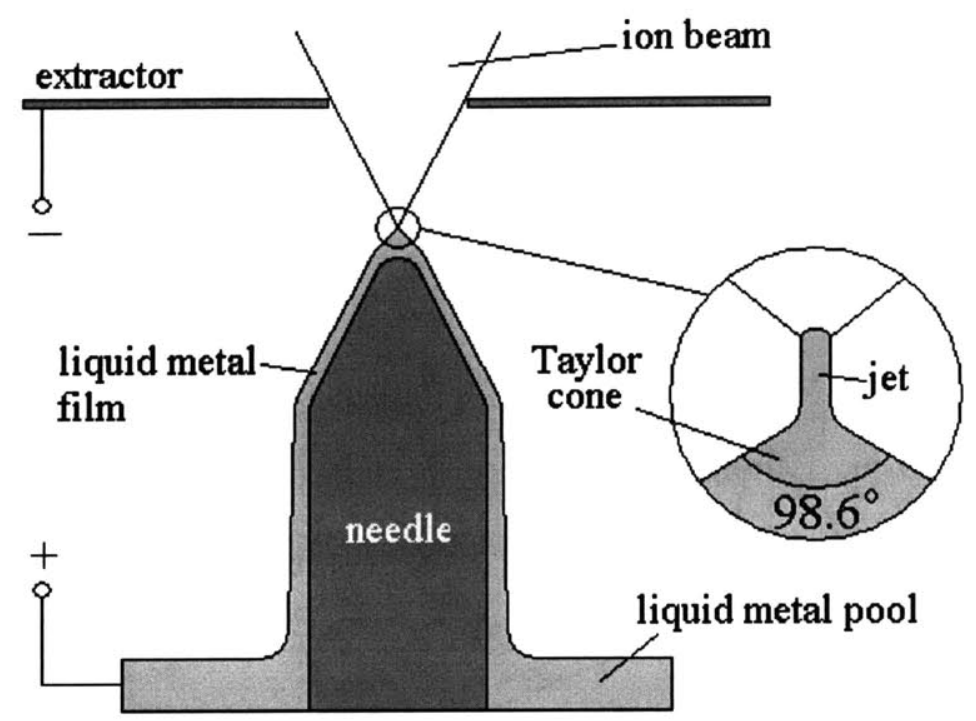

Figure 4.18 Schematic of indium FEEP [Steiger, 2000] (adapted).

In general, the voltage that starts emission is defined as the voltage that yields a $10 \mu \mathrm{A}$ emitted current. Below that threshold voltage required for field emission, current drops off very rapidly and can be neglected. The FEEP unit is characterized by a high power efficiency, which is given by:

$$
\eta_{p}=\frac{\mathrm{V}_{e}\left(I_{e}-I_{a}\right)}{\mathrm{V}_{e} I_{e}-\mathrm{V}_{a} I_{a}}
$$

where $V_{e}$ is the emitter voltage, $V_{a}$ is the accelerator voltage, $I_{e}$ is the emitter current and $I_{a}$ is the accelerator current. Therefore, the only inefficiencies come from those ions that impinge upon the accelerator plate after being emitted instead of being further accelerated down the potential. This efficiency regularly lies above $90 \%$. The thrust as before is the product of mass flow rate and exhaust velocity and can therefore be written as,

$$
F=I_{e} \sqrt{2 \mathrm{~V}_{e}\left(m_{C s} / e\right)},
$$

where $m_{C s}$ is the atomic mass of a cesium ion and $e$ is the charge of an electron $\left(1.6 \times 10^{-19}\right.$ C). Based on experimental data [Marcuccio, 1998], the emitter current is an exponential function of the total voltage $\left(\mathrm{V}_{\mathrm{e}}+\left|\mathrm{V}_{\mathrm{a}}\right|\right)$ experienced by the liquid metal at the tip of the 
emitter. The specific curve fit used in this study can be found in Appendix C, Equation C.26. So, the thrust for the FEEP thruster is much more strongly dependent on the emitter voltage than the one half power that appears above. Equation 4.23 represents the theoretical thrust level. However, the actual, usable, on-axis thrust level is usually about ten percent less than this because of beam divergence. This practical thrust can be written as

$$
F_{\text {pract }}=F\left(\frac{\sin \alpha}{\alpha}\right)\left(\frac{\sin \beta}{\beta}\right) \text {, }
$$

where $\alpha$ and $\beta$ are the divergence angles of the ion beam in the vertical and horizontal planes, respectively. Typical thrusts range from tenths of micronewtons to a millinewton or more. Specific impulse is defined in the same way as with the colloid thruster and typical values fall in the 4000 to 10,000 second range, depending on the voltage applied. For cesium propellant the expression is simply

$$
I_{s p}=122.3 \sqrt{\mathrm{V}_{e}} \text {. }
$$

Recent long duration testing of a cesium FEEP thruster provides some previously untested, useful characteristics, including steady-state thrust variation figures [Marcuccio, 2000]. Two metrics are defined to express the thrust variation:

$$
\begin{aligned}
& D F_{u}=100 \frac{F_{\text {max }}-F_{\text {avg }}}{F_{\text {avg }}} \\
& D F_{d}=100 \frac{F_{\text {avg }}-F_{\text {min }}}{F_{\text {avg }}}
\end{aligned}
$$

where $F_{\text {avg }}$ is the mean thrust over the entire testing period (which were 5 and 3 hours, respectively, for the first and second operating point) and $F_{\min }$ and $F_{\max }$ are defined simply as the minimum and maximum thrust readings, respectively. The values they recorded are shown in Table 4.4. 
TABLE 4.4 FEEP life test data, highlighting steady-state thrust variation [Marcuccio, 2000].

\begin{tabular}{|c|c|c|}
\hline & Operating Point 1 & Operating Point 2 \\
\hline \hline Duration & 5 hours & 3 hours \\
\hline Thrust & $96.7 \mu \mathrm{N}$ & $46.9 \mu \mathrm{N}$ \\
\hline Emitter Voltage & $9.0 \mathrm{kV}$ & $6.6 \mathrm{kV}$ \\
\hline Accelerator Voltage & $-2.0 \mathrm{kV}$ & $-3.5 \mathrm{kV}$ \\
\hline Emitter current (avg) & $0.687 \mathrm{~mA}$ & $0.388 \mathrm{~mA}$ \\
\hline $\mathrm{DF}_{\mathrm{u}}$ & $2.8 \%$ & $5.3 \%$ \\
\hline $\mathrm{DF}_{\mathrm{d}}$ & $2.4 \%$ & $2.3 \%$ \\
\hline
\end{tabular}

\subsubsection{The FEEP Thruster Dynamic Effects Model}

Modeling of the FEEP system is rather different than modeling the other systems included in this study. This results from the fact that the FEEP system is a continuously throttleable propulsion unit with no known non-idealities, aside from the noise noted in Table 4.4. However, the frequency content of this noise is not known to the author. So, the FEEP model uses data available from tests performed on the indium FEEP to capture the effect of this noise. It is not incorporated directly into the simulation, only used as a reference afterward to guide performance evaluations.

An effort was made to construct the FEEP model to be as similar to the other models as possible to allow for a meaningful comparison among them. Therefore, the disturbance force has the ability to be varied in the same ways as the other models. In addition, the same kinds of analyses are performed so that results can be compared directly.

To regulate the spacecraft position, the model uses simple proportional derivative control with position and velocity as inputs to determine an appropriate thrust level, which is achieved by variation of voltage, which strongly affects the emitter current as well (see Equation C.26), according to Equation 4.23. The model gives the thruster controller the ability to sense position and velocity, which it uses to maintain the commanded position. The system can, therefore, sense any error through its position and velocity changes in time but cannot compensate for it until one time increment later. 
For the FEEP example analysis, which follows, the piecewise linear, time varying disturbance force has an average value of $7.5 \mu \mathrm{N}$ with a peak value of $8.5 \mu \mathrm{N}$ and a minimum value of $6.5 \mu \mathrm{N}$, the same as the disturbance forces in the other models' examples. The results of this example are shown in Figure 4.19, Figure 4.20, and Figure 4.21, which show the thrust profile, the trajectory, and the thrust PSD as obtained from indium FEEP test data [Steiger, 2000]. The raw data of the thrust noise produced by the indium FEEP system were not available to be integrated with the simulation, so Figure 4.21 will be used to evaluate the spectral content of all the FEEP designs. As can be seen, the trajectory deviates only a very small distance from the desired position because of a short time lag in the control scheme and the PSD has a very small magnitude, indicating the high precision achievable with the FEEP thruster.

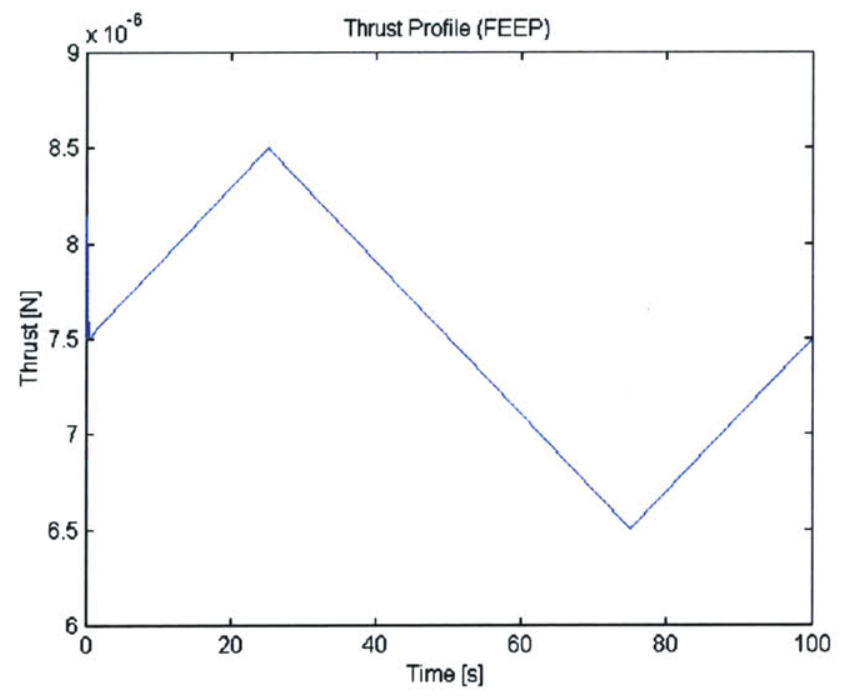

Figure 4.19 FEEP thruster thrust profile example.

\subsubsection{General Attributes and Trends}

The field emission electrostatic propulsion system presents a unique set of characteristics for operational modeling. This is largely because as far as experimentation has shown, there is no impediment to providing thrust at any level over a very broad range without 


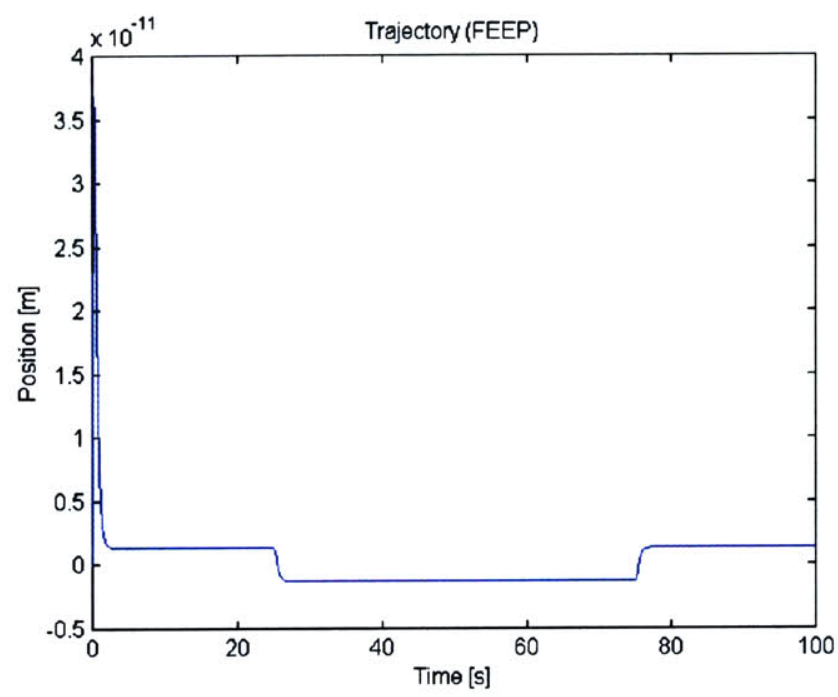

Figure 4.20 FEEP thruster trajectory example.

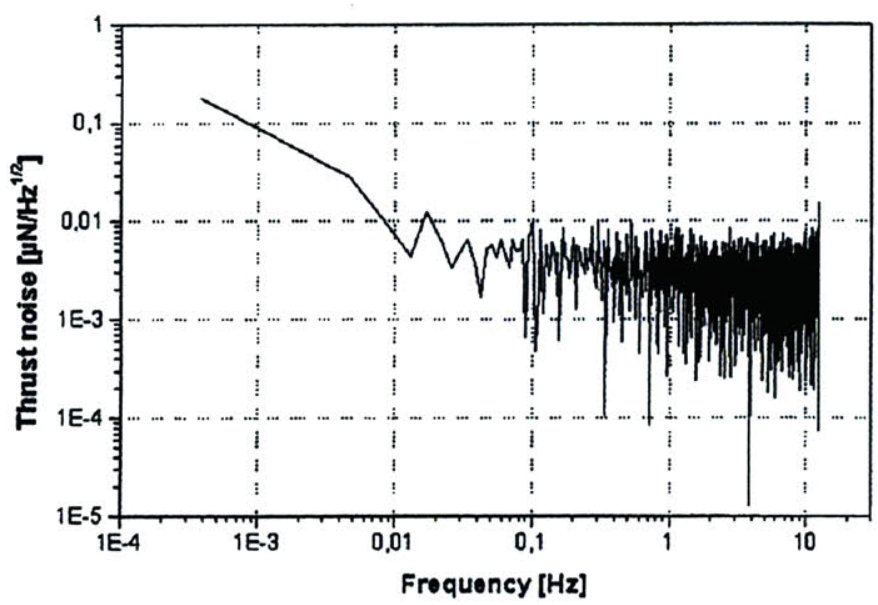

Figure 4.21 FEEP thruster thrust PSD [Steiger, 2000].

significant lags or other non-idealities. Therefore, dynamic modeling is not performed in this study. Only a representation of the thrust noise, a physical result, not an operational result, is considered because it is the largest effect mentioned in any of the recent literature concerning FEEP thrusters. It is likely, because of its high precision operation, that the only areas in which FEEP systems could fall short of other systems are power and space- 
craft interaction (both chemical and electrical), which will be evaluated in mission scenarios where it is a necessary metric for selecting the propulsion system.

\subsection{Cold Gas Thruster}

\subsubsection{Background \& Fundamentals}

The cold gas thruster is a much simpler piece of hardware than the other three propulsion technologies discussed. It is a well understood device and its performance is therefore limited almost exclusively by the inherent propellant properties and scaling issues. Thrust is created by making a compressed gas (usually stored in a liquid state) do work by expanding through a nozzle that is rigidly attached to a spacecraft, allowing force to be transmitted. The two main components of the cold gas thruster are the nozzle and the valve, as illustrated in Figure 4.22. Nozzle theory is well understood and can be summa-

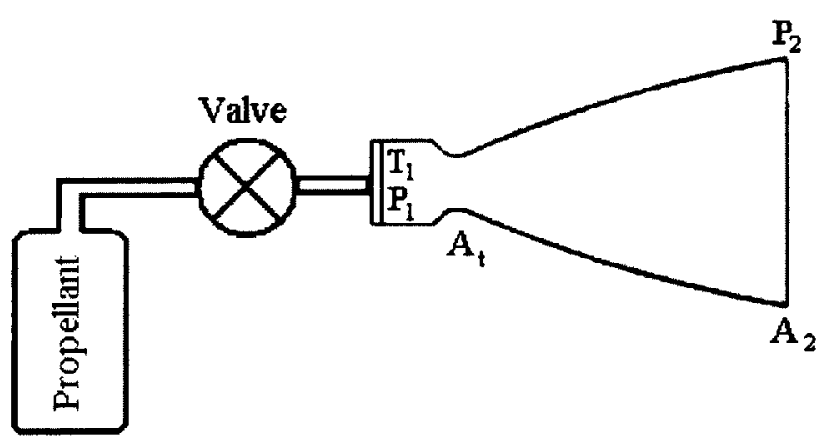

Figure 4.22 Cold gas thruster schematic diagram.

rized by the following quasi-one-dimensional design relations:

$$
\begin{gathered}
\mathrm{v}_{2}=\sqrt{(2 \gamma /(\gamma-1)) R T_{1}\left[1-\left(p_{2} / p_{1}\right)^{(\gamma-1) / \gamma}\right]} \\
M_{i}=\frac{\mathrm{v}_{i}}{\sqrt{\gamma R T_{i}}}
\end{gathered}
$$




$$
\begin{gathered}
\frac{A_{2}}{A_{t}}=\frac{1}{M_{2}}\left(\frac{1+\frac{\gamma-1}{2} M_{2}^{2}}{1+\frac{\gamma-1}{2}}\right)^{\frac{(\gamma+1)}{2(\gamma-1)}} \\
C_{F}=\sqrt{\frac{2 \gamma^{2}}{\gamma-1}\left(\frac{2}{\gamma+1}\right)^{(\gamma+1) /(\gamma-1)}\left[1-\left(p_{2} / p_{1}\right)^{(\gamma-1) / \gamma}\right]}+\frac{p_{2}-p_{3}}{p_{1}} \frac{A_{2}}{A_{t}} \\
F=C_{F} p_{1} A_{t},
\end{gathered}
$$

where the variables are those used conventionally and are listed in the nomenclature section. Common propellants include nitrogen, carbon dioxide and less often, helium. One of the main drawbacks of these simple thrusters is their very low specific impulse, which generally ranges from about 40 to 65 seconds (except for helium, which is approximately 150 seconds). For missions with large $\Delta v$ requirements, the mass fraction of propellant can become prohibitive.

Valves, on the other hand, are more complicated and their future level of performance is harder to approximate, because of recent developments in micro-electro-mechanical systems (MEMS) technology. However, the single most important characteristic is likely to be the minimum open time, which is now typically no less than about 10 milliseconds. Recently, Moog, Inc. has developed a miniature thruster valve that has a minimum 'on' time of less that 2 milliseconds [Bzibziak, 2000]. Coupled with thrusters producing around 5 millinewtons of thrust, cold gas thrusters may soon have the ability to produce minimum impulse bits on the order of 10 to $20 \mu \mathrm{Ns}$. Table 4.5 shows some performance specifications for an existing Moog cold gas thruster. At the operating point given therein, the impulse bit of this thruster can be approximated at $80 \mu \mathrm{Ns}$.

\subsubsection{The Cold Gas Dynamic Effects Model}

The cold gas thruster operational model is similar to the PPT model in that the major mode of operation is in the pulsed regime. This results from the small disturbance force envi- 
TABLE 4.5 Moog cold gas microthruster specification [Bzibziak, 2000].

\begin{tabular}{|c|c|}
\hline & MOOG 58E143 Thruster \\
\hline \hline Operating Pressure & $0-36 \mathrm{psia}$ (regulated) \\
\hline Thrust & $16 \mathrm{mN} @ 21.75 \mathrm{psia}$ \\
\hline Response Time & $2.5 \mathrm{~ms}$ (opening) \\
& $2.5 \mathrm{~ms}$ (closing) \\
\hline Cycle Life & $5 \times 10^{5}>2 \times 10^{6}$ \\
\hline Internal Leakage & $<1 \mathrm{scc} / \mathrm{hr} \mathrm{GN}_{2} @ 36 \mathrm{psia}$ \\
\hline Mass & $40 \mathrm{~g}$ \\
\hline
\end{tabular}

ronment and the relatively high thrust levels of the cold gas thrusters. The only way to deliver small impulses is to fire them for very short intervals. The method of pulsing cold gas thrusters is somewhat less restricted than that of a PPT, however. While, in general, a specific cold gas thruster has a single thrust magnitude, the pulse width is not fixed as it is with a PPT. Therefore, the pulse width $\left(\mathrm{w}_{\mathrm{p}}\right)$ is another parameter, in addition to PRF, that can be varied to match a disturbance force more accurately.

The pulse width is determined by looking at the recent, past disturbance force data and current velocity data. It is defined as the ratio of the impulse imparted to the spacecraft to the net force on the spacecraft. The imparted impulse is the sum of the momentum possessed by the spacecraft at the current time and the impulse imparted by the disturbance force in traversing the dead-band. The pulsing logic is configured to keep the spacecraft within a pre-specified dead-band by commanding thrusting when the spacecraft reaches the dead-band boundary. Table 4.6 lists the parameters used to create the example plots, which follow. These parameters are similar to those used in the PPT example analysis.

TABLE 4.6 Simulation parameters for cold gas thruster example analysis.

\begin{tabular}{|c|c|c|c|c|c|c|}
\hline & Thrust & $\begin{array}{c}\text { Impulse } \\
\text { Bit }\end{array}$ & $\begin{array}{c}\text { Avg. } \\
\text { Disturbance }\end{array}$ & $\begin{array}{c}\text { Dead- } \\
\text { Band }\end{array}$ & $\begin{array}{c}\text { Min. Pulse } \\
\text { Separation }\end{array}$ & S/C Mass \\
\hline \hline Value & $5 \mathrm{mN}$ & $50 \mu \mathrm{Ns}$ & $7.5 \mu \mathrm{N}$ & $.45 \mu \mathrm{m}$ & $10 \mathrm{~ms}$ & $200 \mathrm{~kg}$ \\
\hline
\end{tabular}


However, the maximum PRF is not used as a parameter for the cold gas thruster analysis because it is dependent on the pulse width, which is variable. Instead, the minimum separation time between the end of one pulse and the beginning of the next is used. In general, if the pulses are required to be that close to each other, the thruster was likely undersized for the disturbance force, or the dead-band width is too stringent for the impulse bit. In the regime encountered with the above parameters this limit is not reached, as can be seen in the plot of thrust versus time shown in Figure 4.23. Based on the disturbance force, which

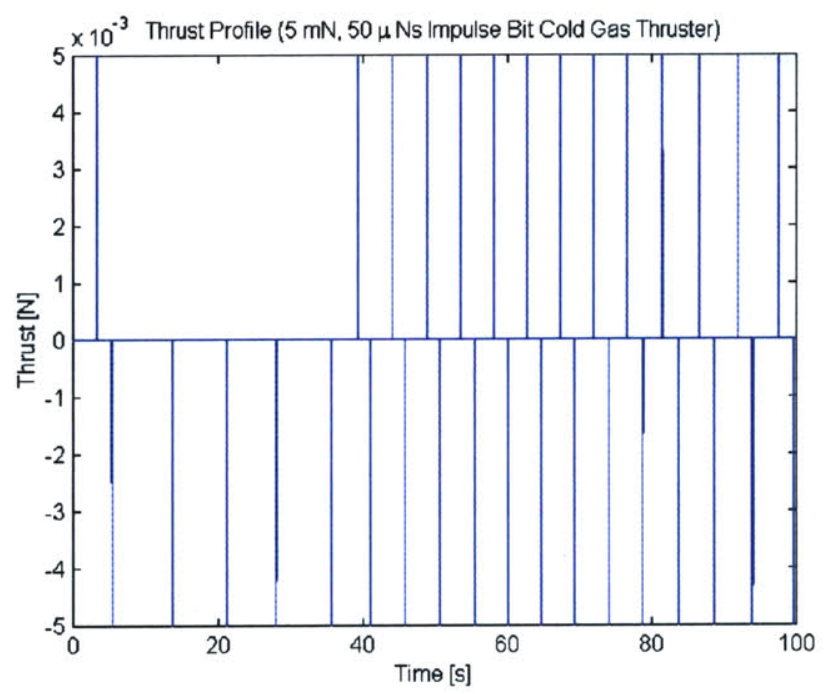

Figure 4.23 Thrust profile for cold gas thruster position maintenance example.

is the same one as those used for the other simulations, and this thrust profile, the trajectory, and acceleration and position power spectral densities are as depicted in Figure 4.24, Figure 4.25 and Figure 4.26, respectively. It is seen in Figure 4.24 that the spacecraft does not always travel the same distance across the dead band. The spacecraft travels a shorter distance in the region near the beginning of the simulation in this case because of the fluctuation in the disturbance force. During this period, the spacecraft logic imparted less impulse than was necessary to cross the dead-band because the disturbance force was 


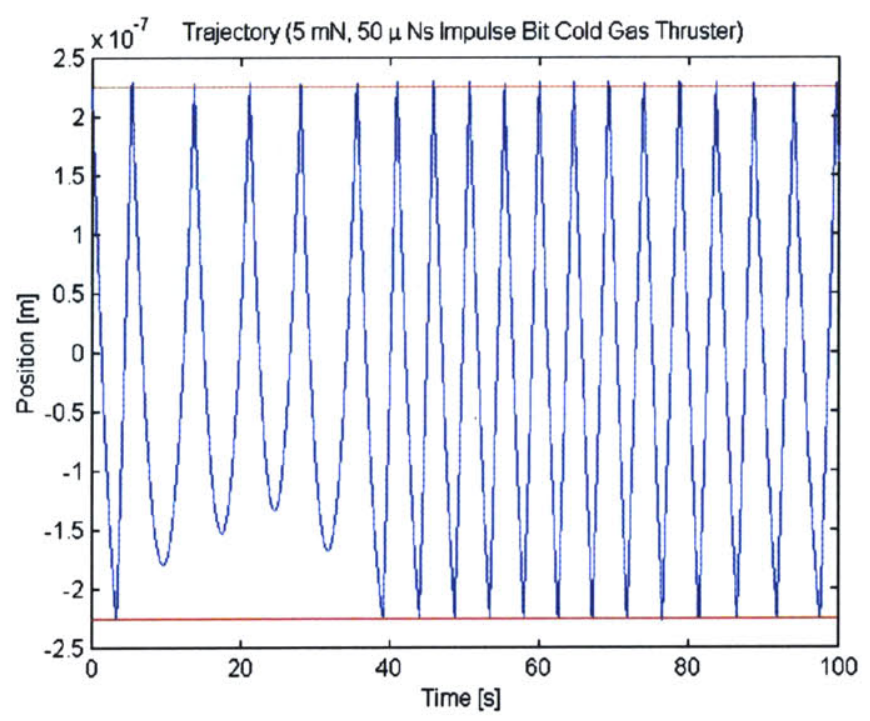

Figure 4.24 Cold gas thruster trajectory example.

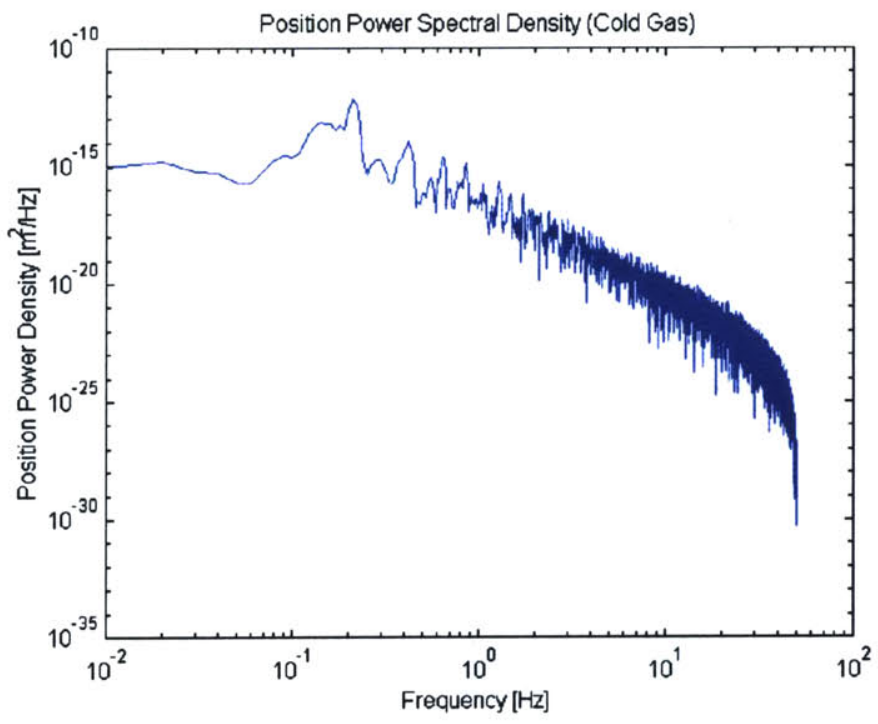

Figure 4.25 Cold gas thruster position PSD example.

stronger than the estimates based on the recent history. Similarly, over-thrusting occurs after this period because the logic is basing the amount of impulse needed on the time period when the disturbance force was larger. For this reason, the spacecraft enters a situation in which the thrusters are continually compensating for each other's over-thrusting. 


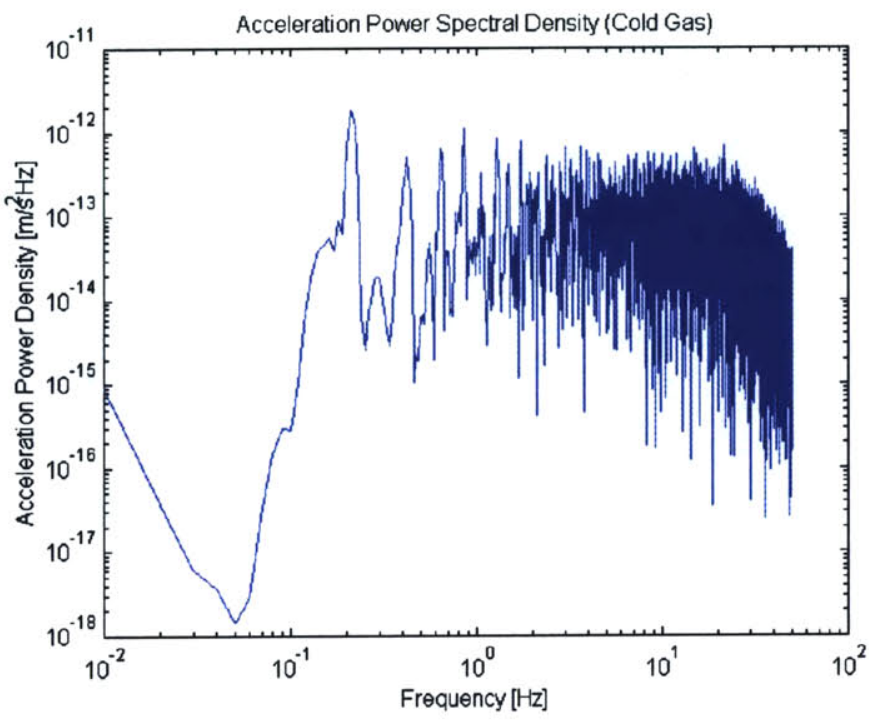

Figure 4.26 Cold gas thruster acceleration PSD example.

While, the spacecraft can maintain the desired dead-band, it wastes a large a mount of fuel in the process.

As is seen in Figure 4.26 the acceleration has a very broadband spectral characteristic because it, like the PPT delivers sharp thrust pulses. On the other hand, the position signal has only a relatively narrow band at low frequencies because the relationship between the dead-band width, the disturbance force and the thruster size allows the spacecraft to traverse the dead-band over a period of many seconds. The trajectory, Figure 4.24, shows the ability to remain within the specified dead-band requirement with an average error of less than five percent and a maximum, initial, transient error of approximately ten percent.

In order to interpret the model and validate some of its results a simple non-dimensional analysis is presented. The main parameters of interest and their units are listed in Table 4.7.

TABLE 4.7 Cold gas thruster model variables and units for dimensional analysis

\begin{tabular}{|c|c|c|c|c|c|c|c|}
\hline Parameter & $\Delta \mathrm{X}$ & $\mathrm{F}_{\mathrm{T}}$ & $\mathrm{F}_{\mathrm{D}}$ & $\mathrm{m}$ & $\mathrm{I}_{\mathrm{b}}$ & $\mathrm{T}_{\text {on }}$ & $\mathrm{f}$ \\
\hline Units & $m$ & $k g \cdot m \cdot s^{-2}$ & $k g \cdot m \cdot s^{-2}$ & $k g$ & $k g \cdot m \cdot s^{-1}$ & $s$ & $s^{-1}$ \\
\hline
\end{tabular}


Given the seven variables and the three dimensions there are four independent dimensionless parameters which characterize this system. One useful group of four parameters is as follows, beginning with equation 4.32 .

$$
\begin{gathered}
\frac{F_{T}}{F_{D}} \\
\frac{t_{o n} f}{f I_{b i t}} \\
F_{D} \\
\frac{m \Delta x f^{2}}{F_{D}}
\end{gathered}
$$

where $\mathrm{F}_{\mathrm{T}}$ is the propulsion unit's thrust level, $\mathrm{F}_{\mathrm{D}}$ is the magnitude of the disturbance force, $t_{o n}$ is the thrust pulse width, $f$ is one over the average time between the beginning of one pulse and the next, $I_{b i t}$ is the impulse bit, $m$ is the spacecraft mass, and $\Delta x$ is the dead-band width. These parameters each have some usefulness in characterizing the system and in particular its stability. Parameter 4.32 gives a first order indication of the suitability of the thruster for the disturbance force. The ratio must be greater than one in order for the system to be effective at all. Parameter 4.33 is the definition of the duty cycle (DC), the fraction of time spent thrusting, which directly impacts the amount of propellant consumed. Parameter 4.35 can be viewed as a ratio of the velocity increment provided by the thruster to the velocity increment imparted by the disturbance force over the period of one thrusting cycle. Using these dimensionless parameters in combination with the physical constraints of the system one can find conditions for stability. From the simple kinematic equation:

$$
x=\frac{1}{2} \frac{F}{m} t^{2}
$$

we can arrive at the approximate dead-band maintenance stability criterion: 


$$
\frac{m \Delta x f^{2}}{F_{D}} \geq \frac{1}{2}
$$

when we interpret the time period to be the inverse of the frequency and the distance traveled to be the dead-band width. When Equation 4.37 is combined with the design requirement,

$$
\frac{\bar{F}}{F_{D}}=\frac{f I_{b i t}}{F_{D}} \sim 1,
$$

that says that average thrust supplied should be very close to the average disturbance force imparted to the system, during a period of time consisting of the sum of the pulse width and the off time before the next pulse, we come up with a more general stability criterion which guides the sizing of the impulse bit of the thruster for a desired dead-band width, given the forcing environment and spacecraft mass:

$$
\frac{I_{b i t}^{2}}{F_{D} m \Delta x} \sim 2
$$

This result can be shown to be generally applicable using the simulation described herein, the source code of which is found in Appendix A, Section A.4.

\subsubsection{General Attributes and Trends}

One of the main drawbacks to using cold gas thrusters for precision formation flying is that their minimum impulse bit is often not small enough, given the current technological state of the hardware. In order to decide whether or not continued time and other resources should be invested in these thrusters, a quick scaling exercise was performed, to determine whether miniaturizing valves further yields an inherent advantage or disadvantage, for a generic solenoid valve. A schematic diagram of the solenoid and plunger is shown in Figure 4.27. The magnetic field inside a solenoid can is shown to be: 


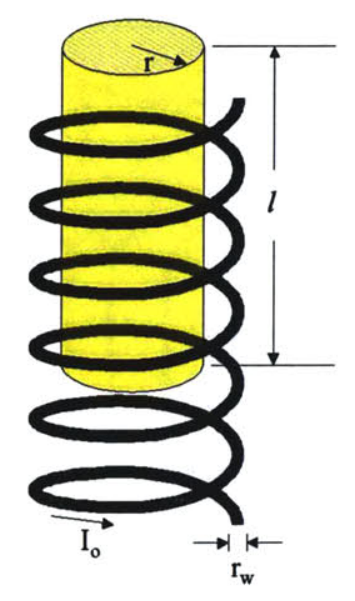

Figure 4.27 Schematic drawing of solenoid and plunger used in scaling analysis

$$
\begin{gathered}
\int(\vec{B} \cdot d \vec{s})=\mu_{o} I_{e n c l} \\
B=\mu_{o} I_{o} n
\end{gathered}
$$

where $\mathrm{B}$ is the magnetic field strength, $\mu_{\mathrm{o}}$ is the permeability of free space, $\mathrm{n}$ is the number of turns of wire per unit length in the solenoid and $I_{0}$ is the current through that wire. From the equation for the magnetic work done to retract the plunger we get the expression for the magnetic force:

$$
\frac{d W_{m}}{d y}=\frac{B^{2}}{2 \mu_{o}} A=\frac{\pi \mu_{o} I_{o}{ }^{2} n^{2} r^{2}}{2}=\left.F_{\Phi}\right|_{y}
$$

where $\mathrm{W}_{\mathrm{m}}$ is the magnetic work in the $\mathrm{y}$ direction (the solenoid axial direction), $\mathrm{A}$ is the cross sectional area inside the solenoid (or the cross sectional area of the plunger), $r$ is the plunger radius and $\mathrm{F}_{\Phi}$ is the magnetic force. Now, assuming that we require the temperature rise, $\Delta \mathrm{T}$, during each operating interval, $\mathrm{t}$, to remain at a prescribed level, in order to prevent overheating, no matter what its size, we write: 


$$
\begin{array}{r}
\text { Constant }=\Delta T=\frac{E}{m c}=\frac{P t}{m c} \\
\Delta T=\frac{\left(I_{o}^{2} \rho_{E} \frac{L}{\pi r_{w}^{2}}\right) t}{\left(\rho_{m} l \pi r^{2}\right) c} \\
\frac{\pi \rho_{m} \Delta T c}{\rho_{E}}=\frac{I_{o}^{2} t}{r r_{w}^{3}}
\end{array}
$$

where $\Delta \mathrm{T}$ is temperature change, $\mathrm{E}$ is energy input, $\mathrm{m}$ is the plunger mass, $\mathrm{c}$ the plunger specific heat, $P$ is the power input during the time needed to open the valve, $t, \rho_{m}$ is the mass density of the solenoid plunger, $\rho_{E}$ is the electrical resistivity of the wire, $r_{w}$ is the wire radius and $L$ is the length of wire wrapped around the solenoid, given by

$$
L=\frac{2 \pi r l}{2 r_{w}}
$$

Now, writing the magnetic force as an acceleration and using it to kinematically determine the time required to move the plunger a given distance, $y$, we have:

$$
y=\frac{1}{2} \frac{F}{m} t^{2}=\frac{1}{2} \frac{\left(\pi \mu_{o} I_{o}{ }^{2} n^{2} r^{2} / 2\right)}{\rho_{m} l \pi r^{2}} t^{2}=\frac{\mu_{o} I_{o}^{2} t^{2}}{16 \rho_{m} r_{w}^{2} l}
$$

where all the variables are as defined previously and $l$ is the length of the solenoid plunger. If we now assume that all length scales in equations 4.42 and 4.44 are multiplied by $\mathrm{f}_{\mathrm{sc}}$ $\left(0<\mathrm{f}_{\mathrm{sc}}<1\right)$ in order to photographically reduce the size of the valve we find that the group $\mathrm{I}^{2} \mathrm{t}$ must decrease by a factor of $\mathrm{f}_{\mathrm{sc}}{ }^{4}$ from equation 4.42. Separating the $\mathrm{I}^{2} \mathrm{t}$ group in equation 4.44 from the other $\mathrm{t}$ term we see that the $\mathrm{f}_{\mathrm{sc}}{ }^{4}$ factor which it carries is already balanced by the group $r_{w}^{2} l y$, which cumulatively carries its own factor of $\mathrm{f}_{\mathrm{sc}}{ }^{4}$. Therefore, $\mathrm{t}$, the time necessary to move the plunger the stroke distance, $y$, is not forced to increase or decrease by the photographic shrinking of the valve. There is no inherent advantage or disadvantage, in terms of opening time, afforded by scaling the valve. 
Looking at a pulsing situation in which a steady state $\Delta \mathrm{T}$ is achieved by the balance between the average dissipated pulse power and the heat conducted away, a different result is found. The expression for the power conducted away must be included as:

$$
P f t_{o n}=\alpha K \frac{\Delta T}{l} \pi r^{2}
$$

where $\mathrm{P}$ is the instantaneous power required to operate the valve, $f$ is the frequency at which the valve is opened, $t_{o n}$ is the duration for which the valve is kept open during each period, $\mathrm{k}$ is the thermal conductivity of the poppet, $\Delta \mathrm{T}$ is solenoid temperature change, and $\mathrm{r}$ and $l$ are dimensions of the solenoid. This new thermal balance requires that

$$
I_{o}^{2} \sim \frac{f_{s c}^{2}}{f t_{o n}}
$$

which is then substituted into Equation 4.44. The resulting scaling law is written as

$$
f_{s c}^{2}-\frac{t^{2}}{f t_{o n}}
$$

Therefore, we see that if the time to open the valve is to remain constant with miniaturization, according to the previous analysis, then the quantity $f t_{o n}$, which is defined as the duty cycle, must increase to keep the temperature difference constant. However, the temperature difference need not be as large as that of the larger valve. So, the result of scaling down the valve is that it can be operated more rapidly (for the same on time) or with larger on-times (for the same frequency), or some combination of the two, because heat conduction is more efficient at smaller scales. 


\section{Chapter 5}

\section{PROPULSION SYSTEM DESIGNS}

This chapter describes the processes adopted in creating the nominal designs, which are also presented here, for each propulsion system for the five example missions. While the following designs are not necessarily optimal, it is felt that for each mission the four propulsion systems are designed with the same degree of rigor and attention to the requirements so that they can be compared fairly.

\subsection{Design Methodologies}

Each thruster design is created according to a selected conglomeration of the thruster physics, operational characteristics and available empirical data. Accordingly, the four design methodologies vary to some degree and as a result have the ability to highlight the advantages and disadvantages of the propulsion systems in the following mission scenarios. The equations, relationships and rules that determine the four propulsion system design methodologies are explained in detail and referenced in Appendix $\mathrm{C}$, which begins on page 201 .

\subsubsection{PPT Methodology}

A flow diagram that outlines the process used to arrive at the pulsed plasma thruster propulsion system designs is shown in Figure 5.1. The parameters shown in red PRF $_{\max }$ and $\Delta v)$ are constrained by either the mission requirements or thruster technology limitations, 
while the blue parameters $\left(\mathrm{I}_{\mathrm{bit}}\right)$ are those which the designer has some freedom to choose. The symbols used are defined in the nomenclature on page 19. The selection of the impulse bit is guided by the simple scaling laws discussed in Section 4.1.3 and the performance results from the thruster simulation. Most of the relationships between the parameters of the PPT are curve fits to empirical data. The variables that appear in the mass summation at the bottom of the figure and do not appear in the flow diagram are assumed to be fixed quantities for the range of designs to be covered. They include the discharge initiation (spark-plug) circuit mass $\left(\mathrm{m}_{\mathrm{dic}}\right)$ and the structural mass $\left(\mathrm{m}_{\mathrm{s}}\right)$ of the thruster.

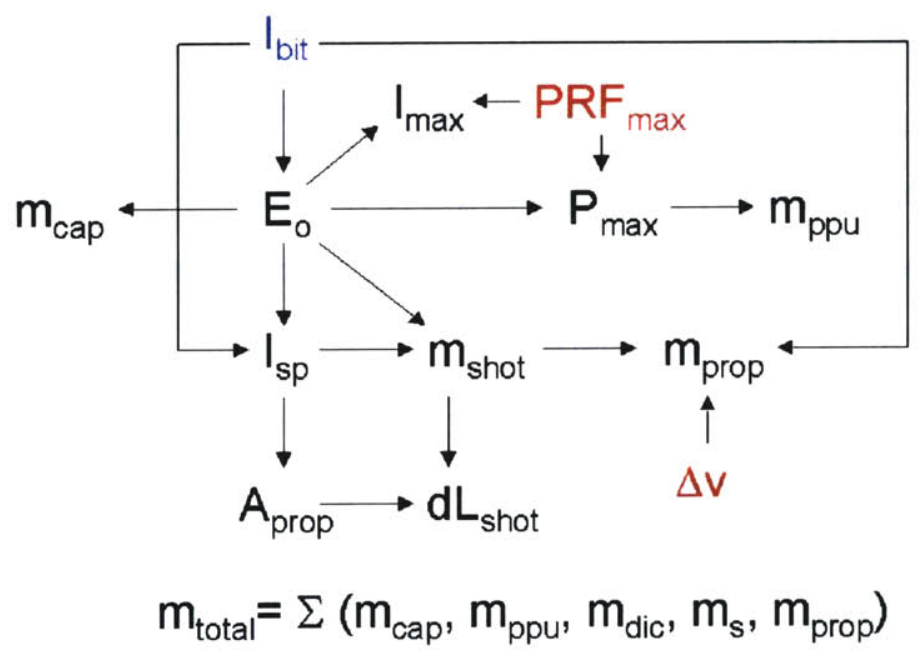

Figure 5.1 PPT design methodology flow diagram.

\subsubsection{Colloid Thruster Methodology}

The flow diagram that outlines the process used to arrive at the colloid thruster propulsion system designs is shown in Figure 5.2. The color coding in the diagram has the same meaning as in the PPT design methodology. The component masses are extrapolations based largely on a sample colloid thruster design from Busek Co., Inc. [Vincent,1999] [Hruby, 2000]. However, the thruster parameters and their relationships to the thrust, power and propellant mass are mainly based on the fundamental thruster physics. The 
designer has some freedom with selecting $Q, \mathrm{~F}_{\max }$ and $\mathrm{V}_{\max }$ (in blue) and the mission requirements strictly fixes $\Delta \mathrm{v}$ (in red).

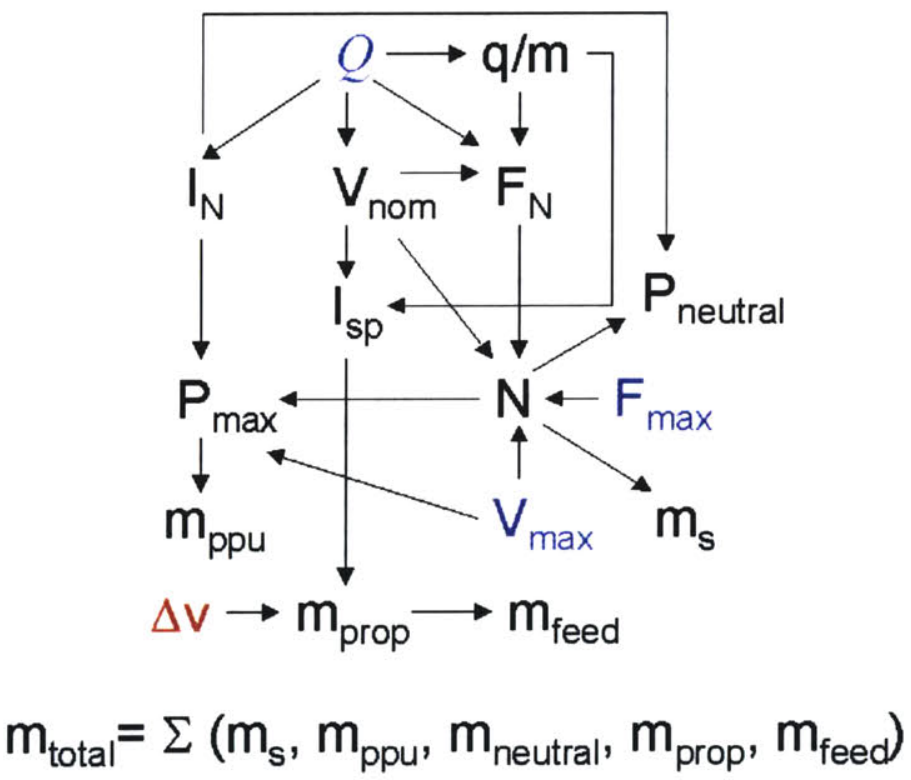

Figure 5.2 Colloid thruster design methodology flow diagram.

\subsubsection{FEEP Thruster Methodology}

A flow diagram that outlines the process used to arrive at the field emission electric propulsion thruster system designs is shown in Figure 5.3. The FEEP designs are based on a combination of analytical expressions and empirical relationships. Although two different FEEP technologies were discussed in Chapter 4, only the cesium based version is used in the design process because of the availability of more information regarding it and the more extensive development of the system. However, the rising popularity of the indium FEEP is duly noted. The two systems are similar enough to provide the correct trends regardless of which one the modeling and design is based on. Again, $\Delta \mathbf{v}$ (red) is fixed and $\mathrm{F}$ and $\mathrm{V}_{\mathrm{a}}$ (red) can be tuned by the designer. 


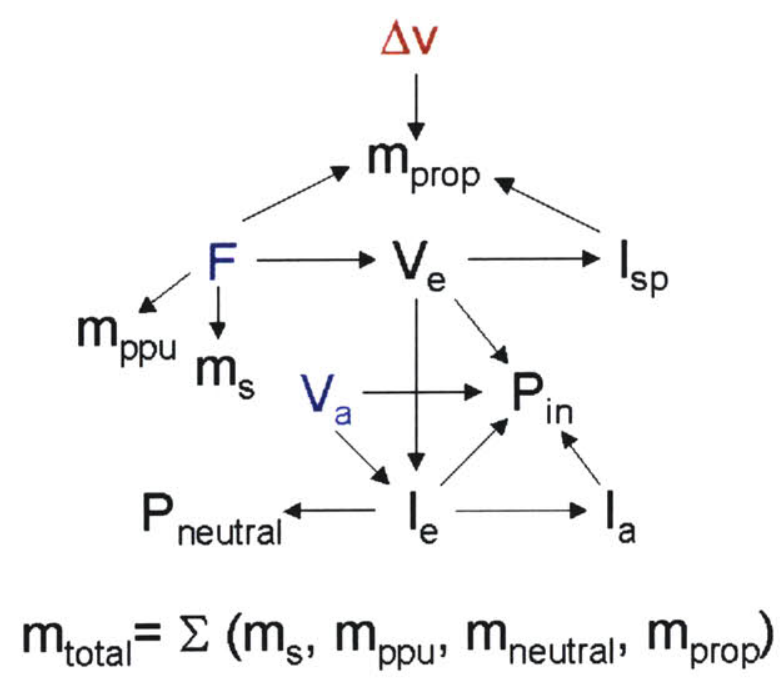

Figure 5.3 FEEP thruster design methodology diagram.

\subsubsection{Cold Gas Thruster Methodology}

A flow diagram that outlines the process used to arrive at the cold gas thruster propulsion system designs is shown in Figure 5.4. This design process is considerably simpler than that of the other systems due in part to a fixed specific impulse and more importantly to some established, available designs and components that do not change very much within the design space explored. While all the parameters are defined in the nomenclature section (page 19), it should be noted here that $p_{1}$ and $p_{\text {tank }}$ represent pressures while $P_{\text {valve }}$ represents a power to avoid confusion in interpreting the diagram. Additionally, $w_{p}$ represents the minimum time for which the thruster valve can be open and with the desired impulse bit determines the size of the thruster. As is represented by the color (red) of the this parameter it is fundamentally limited by the current state of valve technology. Both $\mathrm{I}_{\mathrm{sp}}$ and $\Delta \mathrm{v}$ are also fixed (by propellant and mission requirements, respectively), indicated by their red color, while $\mathrm{I}_{\text {bit }}$ (in blue) can be varied somewhat by the designer. 


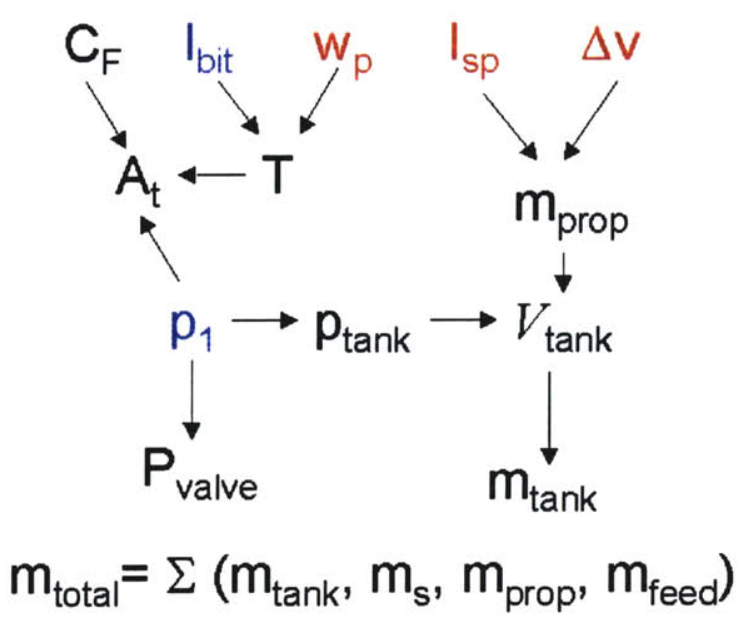

Figure 5.4 Cold gas thruster design methodology diagram.

\subsection{Space Technology 3}

The basic design space for the ST-3 interferometry mission is outlined by the requirements presented in Section 2.2.2. In particular, the two spacecraft (collector and combiner) will need to meet their formation flying requirements while subjected to a solar radiation pressure perturbation estimated to produce an average force of $7.5 \mu \mathrm{N}$. A ten percent variation in this disturbance force is included to represent changes in sunlight-exposed spacecraft area, relative ballistic coefficients of the two spacecraft, reflectance changes and fluctuations of the solar radiation intensity itself. The spacecraft need to maintain their positions to within a box of $10 \mathrm{~cm}$ sides. In the translation to the one dimensional case, an estimated $5.8 \mathrm{~cm}$ dead-band is used. The propulsion system must be able to control all the translational and rotation degrees of freedom of the spacecraft independently. Therefore, a twelve thruster system was chosen to provide this capability in addition to some level of redundancy. A higher level of redundancy is not expected to be necessary for a mission with such a short duration.

Additionally, a $\Delta v$ budget was estimated based on the operational requirements so that propellant masses could be calculated. As a result of the short duration, small spacecraft masses and the modest formation flying requirements (compared to some of the other mis- 
sions), the propulsion systems designed for ST-3 are intended to perform all propulsion functions including precision formation flying and all formation reorientations, which require higher thrust levels in order to be completed within the time constraints set for the mission. For the collector spacecraft which is expected to do the majority of the reorientation required for the interferometry experiments the total $\Delta v$ budget is $60.4 \mathrm{~m} / \mathrm{s}$. The combiner has a much smaller budget at $3.34 \mathrm{~m} / \mathrm{s}$ because most of its maneuvers require only stationkeeping against solar radiation forces and attitude changes.

\subsubsection{PPT Design}

The data presented for the PPT design for the ST-3 mission, in Table 5.1, is representative of the kind of data compiled for the PPT designs for all of the missions studied. A summary of the variables for this design and their organization will serve as a guide for interpreting the rest of the PPT tables in the chapter. The first column of each table is a list of the relevant design parameters and the units that the values in the following columns are given in. Close attention should be paid to the units designation because the same parameters often have very different values for different missions. In the case of ST- 3 there are two columns following the first, one for each of the two spacecraft. In general, the number of columns indicates how many distinct propulsion systems were designed for a mission. If several spacecraft can share the same propulsion system design, that design is only listed once. The tables generally progress from detailed thruster parameters at the top, to power and mass totals at the bottom.

While the symbols used for each parameter are defined in the nomenclature section at the beginning of the thesis, a brief description of the variables presented for the PPT designs and their usage is thought to be helpful. The first two parameter, impulse bit and discharge capacitor energy capacity are straightforward and define the main characteristics of the thruster. The $\mathrm{PRF}_{\max }$ parameter is an estimate of the maximum pulse repetition frequency necessary for this thruster to perform according to mission requirements. Note that it is an upper bound and may only be encountered during particularly high thrust seg- 
ments of the mission. The following two variables represent power draws of the thruster in the normal, or most frequently encountered, mode and in the maximum pulse repetition frequency mode (highest thrust), respectively. Following those is the specific impulse, which is a measure of the exhaust velocity of the propellant. Next is the mass per shot, $\mathrm{m}_{\text {shot }}$, which is the metric that is employed to determine the propellant usage of the system. This figure is related to the specific impulse by the impulse bit and the gravitational constant, as mentioned in 4.4. Then, the maximum current draw of the thruster from the spacecraft is listed, which depends on the amount of time available for the capacitor to be recharged between firings.

Following the maximum current is a list of various masses of thruster components and some useful totals. The component masses, in the order in which they appear, are the discharge capacitor mass, the power processing unit mass, the discharge initiation circuit (spark plug circuit) mass, the supporting structure mass and the propellant mass. For each thruster there are one of each of the following: capacitor, discharge initiation circuit and supporting structure. For every two thrusters there is a power processing unit. The propellant mass is for an entire spacecraft. Lastly, there are two mass totals, the first is the propulsion system mass for one spacecraft and the second is the total mass of propulsion system components on all of the spacecraft for the mission. For ST-3, the final mass total is simply the sum of the two spacecraft mass totals.

TABLE 5.1 ST3 Pulsed Plasma Thruster Design Specifications

\begin{tabular}{|c|cc|}
\hline Design Variable & Collector & Combiner \\
\hline \hline $\mathrm{I}_{\mathrm{bit}}[\mu \mathrm{Ns}]$ & 300 & 300 \\
$\mathrm{E}_{\mathrm{o}}[\mathrm{J}]$ & 19.1 & 19.1 \\
$\mathrm{PRF}_{\max }[\mathrm{Hz}]$ & 4 & 4 \\
$\mathrm{P}_{\text {norm }}[\mathrm{W}]$ & 0.49 & 0.52 \\
$\mathrm{P}_{\max }[\mathrm{W}]$ & 76.2 & 76.2 \\
$\mathrm{I}_{\mathrm{sp}}[\mathrm{s}]$ & 930 & 930 \\
$\mathrm{~m}_{\text {shot }}[\mu \mathrm{g}]$ & 32.9 & 32.9 \\
$\mathrm{I}_{\max }[\mathrm{A}]$ & 3.2 & 3.2 \\
\hline
\end{tabular}


TABLE 5.1 ST3 Pulsed Plasma Thruster Design Specifications

\begin{tabular}{|c|cc|}
\hline Design Variable & Collector & Combiner \\
\hline \hline $\mathrm{m}_{\text {cap }}[\mathrm{kg}]$ & 0.22 & 0.22 \\
$\mathrm{~m}_{\mathrm{ppu}}[\mathrm{kg}]$ & 0.88 & 0.88 \\
$\mathrm{~m}_{\mathrm{dic}}[\mathrm{kg}]$ & 0.23 & 0.23 \\
$\mathrm{~m}_{\mathrm{s}}[\mathrm{kg}]$ & 0.5 & 0.5 \\
$\mathrm{~m}_{\mathrm{prop}}[\mathrm{kg}]$ & 1.52 & 0.11 \\
$\mathrm{~m}_{\text {total } / \mathrm{sc}}[\mathrm{kg}]$ & 18.1 & 16.7 \\
$\mathrm{~m}_{\text {total }}[\mathrm{kg}]$ & \multicolumn{2}{|c|}{34.8} \\
\hline
\end{tabular}

\subsubsection{Colloid Thruster Design}

Again, the data for the colloid thruster design for the ST-3 mission, in Table 5.2, is representative of the kind of data compiled for the colloid thruster designs for all of the missions studied. In order to clarify the designs, a summary of the variables for this design and their organization follows.

The first two parameters listed represent the thrust and current produced by a single needle in the thruster array when operated at nominal conditions, where accelerator voltage is equal to the extractor voltage, $V_{n o m}$, the next variable in the table. Following this are the voltages for operating at the most common operating point or normal operating point and the maximum thrust operating point. The normal operating point corresponds to a condition in which the thruster is counteracting a disturbance force at its average value. Next is the maximum thrust, $\mathrm{F}_{\max }$, delivered by the entire thruster array of $\mathrm{N}$ needles when operated at an accelerator voltage of $\mathrm{V}_{\max }$. The following four variables are the current and power for the array when operated in the normal mode and maximum mode, as they have previously been defined. These figures depend on the number of needles being operated to achieve the desired thrust. Also, the neutralizer power, $\mathrm{P}_{\text {neutral }}$, for each array depends on the current being emitted, and therefore the number of needles operating, and the voltage level of the accelerator. Listed are the normal mode and the maximum mode neutralizer powers. Next, are the various propulsion system component masses. They appear in the following order: propellant mass, structural mass, power processing unit mass, feed 
system mass (includes propellant mass in addition to the propellant storage and transfer hardware) and the neutralizer mass. For each thruster there is structural mass and a neutralizer mass associated. For each pair of thrusters there is a power processing unit. However, there is a single feed system for each spacecraft. Finally, the power and mass totals for the colloid thruster systems are listed for both the normal and maximum modes. The total power figures include the power to operate the emitter and the neutralizer. The mass totals are defined the same way as for the PPT.

TABLE 5.2 ST3 Colloid Thruster Design Specifications

\begin{tabular}{|c|cc|}
\hline Design Variable & Collector & Combiner \\
\hline \hline $\mathrm{F}_{\mathrm{N}}[\mu \mathrm{N}]$ & 0.5 & 0.5 \\
$\mathrm{I}_{\mathrm{N}}[\mathrm{nA}]$ & 294 & 294 \\
$\mathrm{~V}_{\text {nom }}[\mathrm{V}]$ & 2411 & 2411 \\
$\mathrm{~V}_{\text {norm }}[\mathrm{V}]$ & 3617 & 3617 \\
$\mathrm{~V}_{\max }[\mathrm{V}]$ & 4822 & 4822 \\
$\mathrm{~F}_{\max }[\mu \mathrm{N}]$ & 4000 & 4000 \\
$\mathrm{~N}[]$ & 5657 & 5657 \\
$\mathrm{I}_{\text {sp norm }}[\mathrm{s}]$ & 354 & 354 \\
$\mathrm{I}_{\text {norm }}[\mu \mathrm{A}]$ & 3.52 & 3.52 \\
$\mathrm{I}_{\text {max }}[\mathrm{mA}]$ & 1.66 & 1.66 \\
$\mathrm{P}_{\text {norm }}[\mathrm{mW}]$ & 17 & 17 \\
$\mathrm{P}_{\text {max }}[\mathrm{W}]$ & 10.67 & 10.67 \\
$\mathrm{P}_{\text {neutral norm }}[\mathrm{mW}]$ & 1.76 & 1.76 \\
$\mathrm{P}_{\text {neutral max }}[\mathrm{W}]$ & 0.83 & 0.83 \\
$\mathrm{~m}_{\text {prop }}[\mathrm{kg}]$ & 3.46 & 0.24 \\
$\mathrm{~m}_{\mathrm{s}}[\mathrm{kg}]$ & 0.36 & 0.36 \\
$\mathrm{~m}_{\text {ppu }}[\mathrm{kg}]$ & 1.95 & 1.95 \\
$\mathrm{~m}_{\text {feed }}[\mathrm{kg}]$ & 4.54 & 0.69 \\
$\mathrm{~m}_{\text {neutral }}[\mathrm{kg}]$ & 0.03 & 0.03 \\
$\mathrm{P}_{\text {total norm }}[\mathrm{mW}]$ & 18.7 & 18.7 \\
$\mathrm{P}_{\text {total max }}[\mathrm{W}]$ & 11.5 & 11.5 \\
$\mathrm{~m}_{\text {total }}$ sc $[\mathrm{kg}]$ & 20.93 & 17.07 \\
$\mathrm{~m}_{\text {total }}[\mathrm{kg}]$ & & 38.0 \\
\hline & & \\
\hline
\end{tabular}




\subsubsection{FEEP Thruster Design}

A summary of the variables presented for the FEEP thruster system follows. The subscripts 'norm' and 'max' refer to the two operating modes, explained previously, for all variables with which they appear. The 'nom' subscript refers to the operating condition that produces a thrust equal to one half of the maximum thrust level. It is defined in order to serve as a reference point at which different size FEEP systems can be compared. This is because the relationships that determine the mass of various components rely on the thrust (the nominal thrust) of the particular design. After the thrust figures, the emitter voltages, $\mathrm{V}_{\mathrm{e}}$, for the various operational modes are listed, followed by the accelerator voltage, $V_{a}$, which does not change during different operational modes. Both of these voltages are with respect to the cathode, which is at spacecraft potential. Then, the specific impulse, $I_{s p ~ n o r m}$, (determined by $V_{e}$ ) for the normal operating mode is listed. Next, the emitter and accelerator currents are shown for the two operating points. The thrust is based on the emitter current while the accelerator current is the result of a loss mechanism in which some extracted ions impinge on the accelerator grid and leak current to the accelerator power supply. After this, the powers supplied to the thruster itself, $P_{i n}$, and the beam neutralizer, $P_{\text {neutral }}$, are listed. $P_{\text {in }}$ is just the sum of the power to the emitter and the power to accelerator. The component masses are listed next in this order: structural mass, power processing unit mass, neutralizer mass and propellant mass. Similar to the colloid system, the FEEP has a neutralizer and structural mass for each thruster, but only one power processing unit for each pair of thrusters and the propellant mass is that of an entire spacecraft. Finally, the power and mass totals for the propulsion system are shown. They are defined the same way as those in the colloid thruster tables.

TABLE 5.3 ST3 FEEP System Design Specifications

\begin{tabular}{|c|cc|}
\hline Design Variable & Collector & Combiner \\
\hline \hline $\mathrm{F}_{\text {nom }}[\mathrm{mN}]$ & 2 & 2 \\
$\mathrm{~F}_{\max }[\mathrm{mN}]$ & 4 & 4 \\
$\mathrm{~V}_{\mathrm{e} \text { nom }}[\mathrm{V}]$ & 5367 & 5367 \\
$\mathrm{~V}_{\text {e norm }}[\mathrm{V}]$ & 1037 & 1037 \\
\hline
\end{tabular}


TABLE 5.3 ST3 FEEP System Design Specifications

\begin{tabular}{|c|cc|}
\hline Design Variable & Collector & Combiner \\
\hline \hline $\mathrm{V}_{\mathrm{e} \max }[\mathrm{V}]$ & 5951 & 5951 \\
$\mathrm{~V}_{\mathrm{a}}[\mathrm{V}]$ & -3000 & -3000 \\
$\mathrm{I}_{\text {sp norm }}[\mathrm{s}]$ & 3938 & 3938 \\
$\mathrm{I}_{\mathrm{e} \mathrm{norm}}[\mathrm{mA}]$ & 0.15 & 0.15 \\
$\mathrm{I}_{\mathrm{e} \max }[\mathrm{mA}]$ & 34 & 34 \\
$\mathrm{I}_{\mathrm{a} \text { norm }}[\mu \mathrm{A}]$ & 45.8 & 45.8 \\
$\mathrm{I}_{\mathrm{a} \max }[\mathrm{mA}]$ & 6.8 & 6.8 \\
$\mathrm{P}_{\text {in norm }}[\mathrm{W}]$ & 0.3 & 0.3 \\
$\mathrm{P}_{\text {in max }}[\mathrm{W}]$ & 222.6 & 222.6 \\
$\mathrm{P}_{\text {neutral norm }}[\mathrm{mW}]$ & 76.4 & 76.4 \\
$\mathrm{P}_{\text {neutral max }}[\mathrm{W}]$ & 17 & 17 \\
$\mathrm{~m}_{\mathrm{s}}[\mathrm{kg}]$ & 1.52 & 1.52 \\
$\mathrm{~m}_{\text {ppu }}[\mathrm{kg}]$ & 1.29 & 1.29 \\
$\mathrm{~m}_{\text {neutral }}[\mathrm{kg}]$ & 0.03 & 0.03 \\
$\mathrm{~m}_{\text {prop }}[\mathrm{kg}]$ & 0.31 & 0.02 \\
$\mathrm{P}_{\text {total norm }}[\mathrm{W}]$ & 0.37 & 0.37 \\
$\mathrm{P}_{\text {total max }}[\mathrm{W}]$ & 239.6 & 239.6 \\
$\mathrm{~m}_{\text {total } / \mathrm{sc}}[\mathrm{kg}]$ & 26.7 & 26.41 \\
$\mathrm{~m}_{\text {total }}[\mathrm{kg}]$ & & 53.11 \\
\hline
\end{tabular}

\subsubsection{Cold Gas Thruster Design}

The parameters for the cold gas thruster system appear in Table 5.4. The first three variables listed are the impulse bit, thrust and minimum pulse width, $w_{p}$. Given a desired impulse bit and a minimum pulse width, as specified by current information [Bzibziak, 2000 ] on valves that have been developed for very small cold gas thrusters, the necessary thrust provided by the nozzle is determined. Next, the specific impulse (a constant value based on using nitrogen as a propellant) is listed. The following variables are the valve operation power, $\mathrm{P}_{\mathrm{valve}}$, and the average valve power based on the normal operation mode thrusting demands, as determined from the simulation. The value for $P_{\text {valve }}$ was extracted from recent literature discussing miniature cold gas thrusters [Bzibziak, 2000], [Mueller, 
2000]. The component masses are listed after the power and include propellant mass, feed system mass (propellant tank, feed lines, safety valves) and thruster structural mass (nozzle and valve). Lastly, the total masses are shown, with the propulsion system mass for a single spacecraft listed first and the total propulsion systems mass for the entire mission next.

TABLE 5.4 ST3 Cold Gas Thruster Design Specifications

\begin{tabular}{|c|cc|}
\hline Design Variable & Collector & Combiner \\
\hline \hline $\mathrm{I}_{\text {bit }}[\mu \mathrm{Ns}]$ & 200 & 200 \\
$\mathrm{~F}[\mathrm{mN}]$ & 20 & 20 \\
$\mathrm{w}_{\mathrm{p}}[\mathrm{s}]$ & 0.01 & 0.01 \\
$\mathrm{I}_{\mathrm{sp}}[\mathrm{s}]$ & 65 & 65 \\
$\mathrm{P}_{\text {valve }}[\mathrm{W}]$ & 15 & 15 \\
Avg $\mathrm{P}_{\text {valve }}[\mathrm{mW}]$ & 5.4 & 4.9 \\
$\mathrm{~m}_{\text {prop }}[\mathrm{kg}]$ & 18.1 & 1.31 \\
$\mathrm{~m}_{\text {feed }}[\mathrm{kg}]$ & 5.64 & 0.41 \\
$\mathrm{~m}_{\mathrm{s}}[\mathrm{g}]$ & 0.01 & 0.01 \\
$\mathrm{~m}_{\text {total } / \mathrm{sc}}[\mathrm{kg}]$ & 23.9 & 1.84 \\
$\mathrm{~m}_{\text {total }}[\mathrm{kg}]$ & \multicolumn{2}{|c}{25.74} \\
\hline
\end{tabular}

\subsection{TPF}

The design space for the TPF mission is outlined by the requirements presented in Section 2.3.2. In particular, the two different kinds of spacecraft (four collectors and one combiner) will need to meet their formation flying requirements while subjected to a solar radiation pressure perturbation estimated to produce an average force of $300 \mu \mathrm{N}$. A ten percent variation in this disturbance force is included to represent changes in sunlightexposed spacecraft area, relative ballistic coefficients of the two spacecraft and fluctuations of the solar radiation intensity itself. As with the ST-3 mission, the spacecraft need to maintain their positions to within a box of $10 \mathrm{~cm}$ sides. In the translation to the one dimensional case, an estimated $5.8 \mathrm{~cm}$ dead-band requirement is used in the simulations. Additionally, the propulsion system must be able to control all the translational and rota- 
tion degrees of freedom of the spacecraft independently. In this case a sixteen thruster system was chosen to provide this capability in addition to several levels of redundancy because of the extended lifetime of the mission.

A $\Delta v$ budget was estimated based on the operational requirements so that propellant masses could be calculated. The precision formation flying propulsion system designed in this study is charged only with fine formation actuation and not major reorientations or formation rotations. Therefore, the four collector spacecraft are expected to have a total $\Delta v$ budget of $100 \mathrm{~m} / \mathrm{s}$, mainly for stationkeeping to cancel the solar radiation force. The combiner has a smaller budget at $70 \mathrm{~m} / \mathrm{s}$ because most of its smaller area and mass, giving rise to smaller solar radiation forces.

\subsubsection{PPT Design}

TABLE 5.5 TPF Pulsed Plasma Thruster Design Specifications

\begin{tabular}{|c|cc|}
\hline Design Variable & Collector & Combiner \\
\hline \hline $\mathrm{I}_{\mathrm{bit}}[\mu \mathrm{Ns}]$ & 1200 & 1200 \\
$\mathrm{E}_{\mathrm{o}}[\mathrm{J}]$ & 57.8 & 57.8 \\
$\mathrm{PRF}_{\max }[\mathrm{Hz}]$ & 6 & 6 \\
$\mathrm{P}_{\text {norm }}[\mathrm{W}]$ & 15.2 & 15.1 \\
$\mathrm{P}_{\max }[\mathrm{W}]$ & 347.1 & 347.1 \\
$\mathrm{I}_{\mathrm{sp}}[\mathrm{s}]$ & 1375 & 1375 \\
$\mathrm{~m}_{\text {shot }}[\mu \mathrm{g}]$ & 89 & 89 \\
$\mathrm{I}_{\max }[\mathrm{A}]$ & 14.6 & 14.6 \\
$\mathrm{~m}_{\mathrm{cap}}[\mathrm{kg}]$ & 0.66 & 0.66 \\
$\mathrm{~m}_{\mathrm{ppu}}[\mathrm{kg}]$ & 2.24 & 2.24 \\
$\mathrm{~m}_{\mathrm{dic}}[\mathrm{kg}]$ & 0.23 & 0.23 \\
$\mathrm{~m}_{\mathrm{s}}[\mathrm{kg}]$ & 0.7 & 0.7 \\
$\mathrm{~m}_{\mathrm{prop}}[\mathrm{kg}]$ & 1.36 & 0.91 \\
$\mathrm{~m}_{\text {total } / \mathrm{sc}}[\mathrm{kg}]$ & 44.6 & 44.1 \\
$\mathrm{~m}_{\text {total }}[\mathrm{kg}]$ & & 222.5 \\
\hline
\end{tabular}




\subsubsection{Colloid Thruster Design}

TABLE 5.6 TPF Colloid Thruster Design Specifications

\begin{tabular}{|c|cc|}
\hline Design Variable & Collector & Combiner \\
\hline \hline $\mathrm{F}_{\mathrm{N}}[\mu \mathrm{N}]$ & 0.5 & 0.5 \\
$\mathrm{I}_{\mathrm{N}}[\mathrm{nA}]$ & 294 & 294 \\
$\mathrm{~V}_{\text {nom }}[\mathrm{V}]$ & 2411 & 2411 \\
$\mathrm{~V}_{\text {norm }}[\mathrm{V}]$ & 3617 & 3617 \\
$\mathrm{~V}_{\max }[\mathrm{V}]$ & 4822 & 4822 \\
$\mathrm{~F}_{\max }[\mu \mathrm{N}]$ & 900 & 600 \\
$\mathrm{~N}[\mathrm{j}$ & 1273 & 849 \\
$\mathrm{I}_{\text {sp norm }}[\mathrm{s}]$ & 354 & 354 \\
$\mathrm{I}_{\text {norm }}[\mathrm{mA}]$ & 0.14 & 0.14 \\
$\mathrm{I}_{\text {max }}[\mathrm{mA}]$ & 0.37 & 0.25 \\
$\mathrm{P}_{\text {norm }}[\mathrm{W}]$ & 0.69 & 0.69 \\
$\mathrm{P}_{\text {max }}[\mathrm{W}]$ & 2.4 & 1.6 \\
$\mathrm{P}_{\text {neutral norm }}[\mathrm{mW}]$ & 71.9 & 71.9 \\
$\mathrm{P}_{\text {neutral max }}[\mathrm{W}]$ & 0.19 & 0.12 \\
$\mathrm{~m}_{\text {prop }}[\mathrm{kg}]$ & 24.18 & 16.23 \\
$\mathrm{~m}_{\mathrm{s}}[\mathrm{kg}]$ & 0.34 & 0.33 \\
$\mathrm{~m}_{\text {ppu }}[\mathrm{kg}]$ & 0.5 & 0.35 \\
$\mathrm{~m}_{\text {feed }}[\mathrm{kg}]$ & 29.41 & 19.87 \\
$\mathrm{~m}_{\text {neutral }}[\mathrm{kg}]$ & 0.03 & 0.03 \\
$\mathrm{P}_{\text {total norm }}[\mathrm{W}]$ & 0.77 & 0.77 \\
$\mathrm{P}_{\text {total max }}[\mathrm{W}]$ & 2.59 & 1.73 \\
$\mathrm{~m}_{\text {total } / \mathrm{sc}}[\mathrm{kg}]$ & 39.25 & 28.54 \\
$\mathrm{~m}_{\text {total }}[\mathrm{kg}]$ & & 185.54 \\
\hline
\end{tabular}

\subsubsection{FEEP Thruster Design}

TABLE 5.7 TPF FEEP System Design Specifications

\begin{tabular}{|c|cc|}
\hline Design Variable & Collector & Combiner \\
\hline \hline $\mathrm{F}_{\mathrm{nom}}[\mu \mathrm{N}]$ & 450 & 450 \\
$\mathrm{~F}_{\max }[\mu \mathrm{N}]$ & 900 & 900 \\
\hline
\end{tabular}


TABLE 5.7 TPF FEEP System Design Specifications

\begin{tabular}{|c|cc|}
\hline Design Variable & Collector & Combiner \\
\hline \hline $\mathrm{V}_{\text {e nom }}[\mathrm{V}]$ & 5039 & 5039 \\
$\mathrm{~V}_{\mathrm{e} \text { norm }}[\mathrm{V}]$ & 4702 & 4702 \\
$\mathrm{~V}_{\mathrm{e} \max }[\mathrm{V}]$ & 5619 & 5619 \\
$\mathrm{~V}_{\mathrm{a}}[\mathrm{V}]$ & -3000 & -3000 \\
$\mathrm{I}_{\text {sp }}[\mathrm{s}]$ & 8386 & 8386 \\
$\mathrm{I}_{\text {e norm }}[\mathrm{mA}]$ & 2.9 & 2.9 \\
$\mathrm{I}_{\mathrm{e} \max }[\mathrm{mA}]$ & 7.9 & 7.9 \\
$\mathrm{I}_{\text {a norm }}[\mathrm{mA}]$ & 0.57 & 0.57 \\
$\mathrm{I}_{\mathrm{a} \max }[\mathrm{mA}]$ & 1.57 & 1.57 \\
$\mathrm{P}_{\text {in norm }}[\mathrm{W}]$ & 15.21 & 15.21 \\
$\mathrm{P}_{\text {in max }}[\mathrm{W}]$ & 48.91 & 48.91 \\
$\mathrm{P}_{\text {neutral norm }}[\mathrm{W}]$ & 1.43 & 1.43 \\
$\mathrm{P}_{\text {neutral max }}[\mathrm{W}]$ & 3.93 & 3.93 \\
$\mathrm{~m}_{\mathrm{s}}[\mathrm{kg}]$ & 0.69 & 0.69 \\
$\mathrm{~m}_{\text {ppu }}[\mathrm{kg}]$ & 1.05 & 1.05 \\
$\mathrm{~m}_{\text {neutral }}[\mathrm{kg}]$ & 0.1 & 0.1 \\
$\mathrm{~m}_{\text {prop }}[\mathrm{kg}]$ & 1.03 & 0.69 \\
$\mathrm{P}_{\text {total norm }}[\mathrm{W}]$ & 16.64 & 16.64 \\
$\mathrm{P}_{\text {total max }}[\mathrm{W}]$ & 52.84 & 52.84 \\
$\mathrm{~m}_{\text {total } / \mathrm{sc}}[\mathrm{kg}]$ & 20.96 & 20.62 \\
$\mathrm{~m}_{\text {total }}[\mathrm{kg}]$ & & 104.46 \\
\hline
\end{tabular}

\subsubsection{Cold Gas Thruster Design}

TABLE 5.8 TPF Cold Gas Thruster Design Specifications

\begin{tabular}{|c|cc|}
\hline Design Variable & Collector & Combiner \\
\hline \hline $\mathrm{I}_{\mathrm{bit}}[\mathrm{\mu Ns}]$ & 300 & 275 \\
$\mathrm{~F}[\mathrm{mN}]$ & 15 & 13.75 \\
$\mathrm{w}_{\mathrm{p}}[\mathrm{s}]$ & 0.02 & 0.02 \\
$\mathrm{I}_{\mathrm{sp}}[\mathrm{s}]$ & 65 & 65 \\
$\mathrm{P}_{\text {valve }}[\mathrm{W}]$ & 15 & 15 \\
Avg $\mathrm{P}_{\text {valve }}[\mathrm{W}]$ & 0.22 & 0.23 \\
\hline
\end{tabular}


TABLE 5.8 TPF Cold Gas Thruster Design Specifications

\begin{tabular}{|c|cc|}
\hline Design Variable & Collector & Combiner \\
\hline \hline $\mathrm{m}_{\text {prop }}[\mathrm{kg}]$ & 123.5 & 84.4 \\
$\mathrm{~m}_{\text {feed }}[\mathrm{kg}]$ & 38.5 & 26.31 \\
$\mathrm{~m}_{\mathrm{s}}[\mathrm{g}]$ & 0.01 & 0.01 \\
$\mathrm{~m}_{\text {total } / \mathrm{sc}}[\mathrm{kg}]$ & 162.15 & 110.85 \\
$\mathrm{~m}_{\text {total }}[\mathrm{kg}]$ & \multicolumn{2}{|c|}{759.45} \\
\hline
\end{tabular}

\subsection{LISA}

The requirements for the LISA mission are somewhat different from those of the other missions examined in this study. Instead of simple dead-band requirements, limits are placed on the magnitude of position and acceleration noise experienced by the spacecraft is specific frequency bands, as is detailed in Section 2.4.2. However, the environment the three LISA spacecraft will encounter is similar to some of the other missions. In particular, the spacecraft will need to meet their formation flying requirements while subjected to a solar radiation pressure perturbation estimated to produce an average force of $16.75 \mu \mathrm{N}$. Like all the other missions, the simulation for LISA includes a ten percent variation in the average perturbation force experienced to account for fluctuations as discussed above. As with the TPF thruster configuration, each LISA spacecraft is assumed to be outfitted with 16 thrusters allowing for independent motion in all translation planes and rotation axes and multiple levels of redundancy for the several year mission lifetime.

Since the LISA spacecraft are placed in their orbits by independent and ultimately jettisoned propulsion modules, the on-board propulsion system can be designed very specifically for precision formation flying actuation and occasional stationkeeping maneuvers if necessary. As a result the estimated $\Delta v$ budget for each of the spacecraft, almost purely composed of what is necessary to cancel solar radiation pressure, is $4.22 \mathrm{~m} / \mathrm{s}$ for the nominal two year mission or $21.1 \mathrm{~m} / \mathrm{s}$ for the extended 10 year mission lifetime. 


\subsubsection{PPT Design}

TABLE 5.9 LISA Pulsed Plasma Thruster Design Specifications (Note: mass figures in parentheses denote 10 year mission lifetime, those without are for the nominal 2 year mission)

\begin{tabular}{|c|c|}
\hline Design Variable & Value \\
\hline \hline $\mathrm{I}_{\text {bit }}[\mu \mathrm{Ns}]$ & 2 \\
$\mathrm{E}_{\mathrm{o}}[\mathrm{J}]$ & 2.4 \\
$\mathrm{PRF}_{\max }[\mathrm{Hz}]$ & 17 \\
$\mathrm{P}_{\text {norm }}[\mathrm{W}]$ & 24 \\
$\mathrm{P}_{\max }[\mathrm{W}]$ & 40.8 \\
$\mathrm{I}_{\mathrm{sp}}[\mathrm{s}]$ & 157 \\
$\mathrm{~m}_{\text {shot }}[\mu \mathrm{g}]$ & 1.3 \\
$\mathrm{I}_{\mathrm{max}}[\mathrm{A}]$ & 1.7 \\
$\mathrm{~m}_{\text {cap }}[\mathrm{kg}]$ & 0.027 \\
$\mathrm{~m}_{\mathrm{ppu}}[\mathrm{kg}]$ & 0.7 \\
$\mathrm{~m}_{\mathrm{dic}}[\mathrm{kg}]$ & 0.11 \\
$\mathrm{~m}_{\mathrm{s}}[\mathrm{kg}]$ & 0.05 \\
$\mathrm{~m}_{\text {prop }}[\mathrm{kg}]$ & $0.55(2.74)$ \\
$\mathrm{m}_{\text {total }}[\mathrm{sg}]$ & $9.24(11.43)$ \\
$\mathrm{m}_{\text {total }}[\mathrm{kg}]$ & $27.7(34.29)$ \\
\hline \multicolumn{2}{|c}{}
\end{tabular}

\subsubsection{Colloid Thruster Design}

TABLE 5.10 LISA Colloid Thruster Design Specifications (Note: mass figures in parentheses denote 10 year mission lifetime, those without are for the nominal 2 year mission)

\begin{tabular}{|c|c|}
\hline Design Variable & Value \\
\hline \hline $\mathrm{F}_{\mathrm{N}}[\mu \mathrm{N}]$ & 0.13 \\
$\mathrm{I}_{\mathrm{N}}[\mathrm{nA}]$ & 123 \\
$\mathrm{~V}_{\text {nom }}[\mathrm{V}]$ & 2000 \\
$\mathrm{~V}_{\text {norm }}[\mathrm{V}]$ & 3500 \\
$\mathrm{~V}_{\max }[\mathrm{V}]$ & 5000 \\
$\mathrm{~F}_{\max }[\mu \mathrm{N}]$ & 100 \\
$\mathrm{~N}[]$ & 487 \\
$\mathrm{I}_{\text {sp norm }}[\mathrm{s}]$ & 509 \\
$\mathrm{I}_{\text {norm }}[\mu \mathrm{A}]$ & 12 \\
\hline
\end{tabular}


TABLE 5.10 LISA Colloid Thruster Design Specifications (Note: mass figures in parentheses denote 10 year mission lifetime, those without are for the nominal 2 year mission)

\begin{tabular}{|c|c|}
\hline Design Variable & Value \\
\hline \hline $\mathrm{I}_{\max }[\mathrm{\mu A}]$ & 59.7 \\
$\mathrm{P}_{\text {norm }}[\mathrm{mW}]$ & 56.1 \\
$\mathrm{P}_{\max }[\mathrm{W}]$ & 0.4 \\
$\mathrm{P}_{\text {neutral norm }}[\mathrm{mW}]$ & 6.01 \\
$\mathrm{P}_{\text {neutral max }}[\mathrm{mW}]$ & 29.9 \\
$\mathrm{~m}_{\text {prop }}[\mathrm{kg}]$ & $0.17(0.84)$ \\
$\mathrm{m}_{\mathrm{s}}[\mathrm{kg}]$ & 0.33 \\
$\mathrm{~m}_{\mathrm{ppu}}[\mathrm{kg}]$ & 0.14 \\
$\mathrm{~m}_{\text {feed }}[\mathrm{kg}]$ & $0.6(1.41)$ \\
$\mathrm{m}_{\text {neutral }}[\mathrm{kg}]$ & 0.03 \\
$\mathrm{P}_{\text {total norm }}[\mathrm{mW}]$ & 62.1 \\
$\mathrm{P}_{\text {total max }}[\mathrm{W}]$ & 0.43 \\
$\mathrm{~m}_{\text {total } / \mathrm{sc}}[\mathrm{kg}]$ & $7.54(8.35)$ \\
$\mathrm{m}_{\text {total }}[\mathrm{kg}]$ & $22.62(25.05)$ \\
\hline
\end{tabular}

\subsubsection{FEEP Thruster Design}

TABLE 5.11 LISA FEEP System Design Specifications (Note: mass figures in parentheses denote 10 year mission lifetime, those without are for the nominal 2 year mission)

\begin{tabular}{|c|c|}
\hline Design Variable & Value \\
\hline \hline $\mathrm{F}_{\text {nom }}[\mu \mathrm{N}]$ & 16.75 \\
$\mathrm{~F}_{\max }[\mu \mathrm{N}]$ & 100 \\
$\mathrm{~V}_{\mathrm{e} \text { nom }}[\mathrm{V}]$ & 3246 \\
$\mathrm{~V}_{\mathrm{e} \text { norm }}[\mathrm{V}]$ & 3246 \\
$\mathrm{~V}_{\mathrm{e} \max }[\mathrm{V}]$ & 4702 \\
$\mathrm{~V}_{\mathrm{a}}[\mathrm{V}]$ & -3000 \\
$\mathrm{I}_{\mathrm{sp}}[\mathrm{s}]$ & 6967 \\
$\mathrm{I}_{\mathrm{e} \text { norm }}[\mathrm{mA}]$ & 0.19 \\
$\mathrm{I}_{\mathrm{e} \max }[\mathrm{mA}]$ & 0.96 \\
$\mathrm{I}_{\mathrm{a} \operatorname{norm}}[\mu \mathrm{A}]$ & 38.5 \\
$\mathrm{I}_{\mathrm{a} \max }[\mu \mathrm{A}]$ & 191.2 \\
$\mathrm{P}_{\text {in norm }}[\mathrm{W}]$ & 0.74 \\
\hline
\end{tabular}


TABLE 5.11 LISA FEEP System Design Specifications (Note: mass figures in parentheses denote 10 year mission lifetime, those without are for the nominal 2 year mission)

\begin{tabular}{|c|c|}
\hline Design Variable & Value \\
\hline \hline $\mathrm{P}_{\text {in max }}[\mathrm{W}]$ & 5.07 \\
$\mathrm{P}_{\text {neutral norm }}[\mathrm{mW}]$ & 96.3 \\
$\mathrm{P}_{\text {neutral max }}[\mathrm{mW}]$ & 478 \\
$\mathrm{~m}_{\mathrm{s}}[\mathrm{kg}]$ & 0.46 \\
$\mathrm{~m}_{\mathrm{ppu}}[\mathrm{kg}]$ & 0.99 \\
$\mathrm{~m}_{\text {neutral }}[\mathrm{kg}]$ & 0.1 \\
$\mathrm{~m}_{\text {prop }}[\mathrm{kg}]$ & $0.012(0.062)$ \\
$\mathrm{P}_{\text {total norm }}[\mathrm{W}]$ & 0.84 \\
$\mathrm{P}_{\text {total max }}[\mathrm{W}]$ & 5.55 \\
$\mathrm{~m}_{\text {total} / \mathrm{sc}}[\mathrm{kg}]$ & $15.67(15.72)$ \\
$\mathrm{m}_{\text {total }}[\mathrm{kg}]$ & $47.01(47.16)$ \\
\hline
\end{tabular}

\subsubsection{Cold Gas Thruster Design}

TABLE 5.12 LISA Cold Gas Thruster Design Specifications (Note: mass figures in parentheses denote 10 year mission lifetime, those without are for the nominal 2 year mission)

\begin{tabular}{|c|c|}
\hline Design Variable & Value \\
\hline \hline $\mathrm{I}_{\mathrm{bit}}[\mu \mathrm{Ns}]$ & 4.2 \\
$\mathrm{~F}[\mathrm{mN}]$ & 0.42 \\
$\mathrm{w}_{\mathrm{p}}[\mathrm{s}]$ & 0.01 \\
$\mathrm{I}_{\mathrm{sp}}[\mathrm{s}]$ & 65 \\
$\mathrm{P}_{\text {valve }}[\mathrm{W}]$ & 10 \\
$\mathrm{Avg} \mathrm{P}_{\mathrm{valve}}[\mathrm{W}]$ & 0.58 \\
$\mathrm{~m}_{\text {prop }}[\mathrm{kg}]$ & $1.65(8.15)$ \\
$\mathrm{m}_{\text {feed }}[\mathrm{kg}]$ & $0.51(2.54)$ \\
$\mathrm{m}_{\mathrm{s}}[\mathrm{g}]$ & 0.01 \\
$\mathrm{~m}_{\text {total }}[\mathrm{sc}[\mathrm{kg}]$ & $2.33(10.85)$ \\
$\mathrm{m}_{\text {total }}[\mathrm{kg}]$ & $6.99(32.55)$ \\
\hline
\end{tabular}




\subsection{MAXIM}

The proposed architecture for the MAXIM mission is much more massive and has more varied components than other interferometry missions. So, not all components are considered here. The components treated in this study are the 33 collector spacecraft and the detector spacecraft. They experience very different disturbance environments. While both are supposed to deal with solar radiation pressure by way of reflective wings of some sort, they must each deal with different gravity induced perturbations, which arise from their need to follow highly non-'Keplerian' orbits. The detector will need to follow a trajectory as much as $5000 \mathrm{~km}$ below the plane of the ecliptic so that the much more massive converger spacecraft (not modeled) can be in the plane. The collector spacecraft will have to follow orbits up to $10 \mathrm{~km}$ above the plane of the ecliptic. As a result, the collectors will have to fight an ecliptic plane pointing force estimated to be approximately $1.5 \mu \mathrm{N}$ and the detector will have to fight a force of similar nature, but with a magnitude of $198 \mu \mathrm{N}$.

The MAXIM formation flying requirements are estimated at a dead-band of $20 \mathrm{~nm}$, which is then translated to approximately $11.5 \mathrm{~nm}$ in the one dimensional simulation. In order to achieve this each of the collector spacecraft has eight thrusters allowing for decoupled translation in all three directions with some redundancy. The detector spacecraft has six thrusters, but the two operating along the boresight axis are much larger to counteract the gravity induced perturbation toward the plane of the ecliptic.

The $\Delta \mathrm{v}$ budgets for the MAXIM mission are presented as written in the "MAXIM Preliminary Design" document by Webster Cash [Cash, 2000]. These requirements are given as $126 \mathrm{Ns}$ of impulse for the collector spacecraft and the detector in the transverse plane (see Figure 2.6 for clarification of the MAXIM constellation geometry) and 31,540 Ns for the detector spacecraft along the boresight axis over a ten year mission duration. With the rough estimate of the spacecraft's masses at $1000 \mathrm{~kg}$, these impulses simply become velocity increments of $0.126 \mathrm{~m} / \mathrm{s}$ and $31.54 \mathrm{~m} / \mathrm{s}$. 


\subsubsection{PPT Design}

TABLE 5.13 MAXIM Pulsed Plasma Thruster Design Specifications

\begin{tabular}{|c|cc|}
\hline Design Variable & Collector & Detector \\
\hline \hline $\mathrm{I}_{\mathrm{bit}}[\mu \mathrm{Ns}]$ & 6.2 & 89 \\
$\mathrm{E}_{\mathrm{o}}[\mathrm{J}]$ & 5.17 & 9.96 \\
$\mathrm{PRF}_{\max }[\mathrm{Hz}]$ & 4 & 4 \\
$\mathrm{P}_{\text {norm }}[\mathrm{W}]$ & 1.24 & 23.9 \\
$\mathrm{P}_{\max }[\mathrm{W}]$ & 20.7 & 39.8 \\
$\mathrm{I}_{\mathrm{sp}}[\mathrm{s}]$ & 157 & 1111 \\
$\mathrm{~m}_{\mathrm{shot}}[\mu \mathrm{g}]$ & 4 & 8.18 \\
$\mathrm{I}_{\mathrm{max}}[\mathrm{A}]$ & 0.87 & 1.67 \\
$\mathrm{~m}_{\mathrm{cap}}[\mathrm{kg}]$ & 0.06 & 0.11 \\
$\mathrm{~m}_{\mathrm{ppu}}[\mathrm{kg}]$ & 0.6 & 0.7 \\
$\mathrm{~m}_{\mathrm{dic}}[\mathrm{kg}]$ & 0.11 & 0.23 \\
$\mathrm{~m}_{\mathrm{s}}[\mathrm{kg}]$ & 0.05 & 0.5 \\
$\mathrm{~m}_{\text {prop }}[\mathrm{kg}]$ & 0.016 & 1.16 \\
$\mathrm{~m}_{\text {total } / \mathrm{sc}}[\mathrm{kg}]$ & 4.21 & 5.64 \\
$\mathrm{~m}_{\text {total }}[\mathrm{kg}]$ & \multicolumn{2}{|c}{144.57} \\
\hline
\end{tabular}

\subsubsection{Colloid Thruster Design}

TABLE 5.14 MAXIM Colloid Thruster Design Specifications

\begin{tabular}{|c|cc|}
\hline Design Variable & Collector & Detector \\
\hline \hline $\mathrm{F}_{\mathrm{N}}[\mu \mathrm{N}]$ & 0.13 & 0.5 \\
$\mathrm{I}_{\mathrm{N}}[\mathrm{nA}]$ & 123 & 294 \\
$\mathrm{~V}_{\mathrm{nom}}[\mathrm{V}]$ & 2000 & 2411 \\
$\mathrm{~V}_{\text {norm }}[\mathrm{V}]$ & 3500 & 3617 \\
$\mathrm{~V}_{\max }[\mathrm{V}]$ & 5000 & 4822 \\
$\mathrm{~F}_{\max }[\mu \mathrm{N}]$ & 4 & 300 \\
$\mathrm{~N}[]$ & 19 & 424 \\
$\mathrm{I}_{\mathrm{sp} \mathrm{norm}}[\mathrm{s}]$ & 509 & 353 \\
$\mathrm{I}_{\text {norm }}[\mu \mathrm{A}]$ & 1.1 & 94.8 \\
$\mathrm{I}_{\max }[\mu \mathrm{A}]$ & 2.3 & 124.4 \\
\hline
\end{tabular}


TABLE 5.14 MAXIM Colloid Thruster Design Specifications

\begin{tabular}{|c|cc|}
\hline Design Variable & Collector & Detector \\
\hline \hline $\mathrm{P}_{\text {norm }}[\mathrm{mW}]$ & 5.15 & 0.46 \\
$\mathrm{P}_{\max }[\mathrm{mW}]$ & 15.5 & 800 \\
$\mathrm{P}_{\text {neutral norm }}[\mathrm{mW}]$ & 0.55 & 47.4 \\
$\mathrm{P}_{\text {neutral max }}[\mathrm{mW}]$ & 1.2 & 62.2 \\
$\mathrm{~m}_{\text {prop }}[\mathrm{kg}]$ & 0.025 & 9.06 \\
$\mathrm{~m}_{\mathrm{s}}[\mathrm{kg}]$ & 0.33 & 0.33 \\
$\mathrm{~m}_{\mathrm{ppu}}[\mathrm{kg}]$ & 0.076 & 0.21 \\
$\mathrm{~m}_{\text {feed }}[\mathrm{kg}]$ & 0.43 & 11.29 \\
$\mathrm{~m}_{\text {neutral }}[\mathrm{kg}]$ & 0.03 & 0.03 \\
$\mathrm{P}_{\text {total norm }}[\mathrm{mW}]$ & 5.7 & 505 \\
$\mathrm{P}_{\text {total max }}[\mathrm{mW}]$ & 16.7 & 862.3 \\
$\mathrm{~m}_{\text {total } / \mathrm{sc}}[\mathrm{kg}]$ & 3.61 & 13.82 \\
$\mathrm{~m}_{\text {total }}[\mathrm{kg}]$ & \multicolumn{2}{|c}{133.08} \\
\hline
\end{tabular}

\subsubsection{FEEP Thruster Design}

TABLE 5.15 MAXIM FEEP System Design Specifications

\begin{tabular}{|c|cc|}
\hline Design Variable & Collector & Detector \\
\hline \hline $\mathrm{F}_{\text {nom }}[\mu \mathrm{N}]$ & 1.5 & 100 \\
$\mathrm{~F}_{\max }[\mu \mathrm{N}]$ & 4 & 200 \\
$\mathrm{~V}_{\mathrm{e} \text { nom }}[\mathrm{V}]$ & 1425 & 4705 \\
$\mathrm{~V}_{\mathrm{e} \mathrm{norm}}[\mathrm{V}]$ & 1425 & 5271 \\
$\mathrm{~V}_{\mathrm{e} \max }[\mathrm{V}]$ & 2134 & 5280 \\
$\mathrm{~V}_{\mathrm{a}}[\mathrm{V}]$ & -3000 & -3000 \\
$\mathrm{I}_{\mathrm{sp}}[\mathrm{s}]$ & 4617 & 8879 \\
$\mathrm{I}_{\mathrm{e} \text { norm }}[\mathrm{mA}]$ & 0.026 & 1.79 \\
$\mathrm{I}_{\mathrm{e} \max }[\mathrm{mA}]$ & 0.057 & 1.81 \\
$\mathrm{I}_{\mathrm{a} \text { norm }}[\mu \mathrm{A}]$ & 7.8 & 358 \\
$\mathrm{I}_{\mathrm{a} \max }[\mu \mathrm{A}]$ & 17 & 361 \\
$\mathrm{P}_{\text {in norm }}[\mathrm{W}]$ & 0.06 & 10.5 \\
$\mathrm{P}_{\text {in max }}[\mathrm{W}]$ & 0.17 & 10.62 \\
$\mathrm{P}_{\text {neutral norm }}[\mathrm{mW}]$ & 13 & 894 \\
\hline
\end{tabular}


TABLE 5.15 MAXIM FEEP System Design Specifications

\begin{tabular}{|c|cc|}
\hline Design Variable & Collector & Detector \\
\hline \hline $\mathrm{P}_{\text {neutral max }}[\mathrm{mW}]$ & 28.4 & 903 \\
$\mathrm{~m}_{\mathrm{s}}[\mathrm{kg}]$ & 0.447 & 0.5 \\
$\mathrm{~m}_{\mathrm{ppu}}[\mathrm{kg}]$ & 0.985 & 1.00 \\
$\mathrm{~m}_{\text {neutral }}[\mathrm{kg}]$ & 0.03 & 0.03 \\
$\mathrm{~m}_{\text {prop }}[\mathrm{kg}]$ & 0.003 & 0.36 \\
$\mathrm{P}_{\text {total norm }}[\mathrm{W}]$ & 0.073 & 11.39 \\
$\mathrm{P}_{\text {total max }}[\mathrm{W}]$ & 0.2 & 11.52 \\
$\mathrm{~m}_{\text {total } / \mathrm{sc}}[\mathrm{kg}]$ & 7.76 & 6.3 \\
$\mathrm{~m}_{\text {total }}[\mathrm{kg}]$ & \multicolumn{2}{|c}{262.38} \\
\hline
\end{tabular}

\subsubsection{Cold Gas Thruster Design}

TABLE 5.16 MAXIM Cold Gas Thruster Design Specifications

\begin{tabular}{|c|cc|}
\hline Design Variable & Collector & Detector \\
\hline \hline $\mathrm{I}_{\mathrm{bit}}[\mu \mathrm{Ns}]$ & 12 & 135 \\
$\mathrm{~F}[\mathrm{mN}]$ & 1.2 & 13.5 \\
$\mathrm{w}_{\mathrm{p}}[\mathrm{s}]$ & 0.01 & 0.01 \\
$\mathrm{I}_{\mathrm{sp}}[\mathrm{s}]$ & 65 & 65 \\
$\mathrm{P}_{\text {valve }}[\mathrm{W}]$ & 10 & 10 \\
Avg $\mathrm{P}_{\text {valve }}[\mathrm{mW}]$ & 12.1 & 133.3 \\
$\mathrm{~m}_{\text {prop }}[\mathrm{kg}]$ & 0.2 & 48.43 \\
$\mathrm{~m}_{\text {feed }}[\mathrm{kg}]$ & 0.062 & 15.1 \\
$\mathrm{~m}_{\mathrm{s}}[\mathrm{g}]$ & 0.01 & 0.01 \\
$\mathrm{~m}_{\text {total } / \mathrm{sc}}[\mathrm{kg}]$ & 0.34 & 63.59 \\
$\mathrm{~m}_{\text {total }}[\mathrm{kg}]$ & \multicolumn{2}{|c}{74.81} \\
\hline
\end{tabular}

\subsection{SPECS}

The SPECS architecture is unique from all the other missions because the three spacecraft that need to perform precision formation flying are tethered to a larger rotating truss structure (whose propulsion requirements are not analyzed in this study). Still, they are disturbed from their desired positions by solar radiation pressure like the other mission, but 
given the estimated size of the three apertures the average force is expected to be approximately $45 \mu \mathrm{N}$. Again, a ten percent variation in this disturbance force is included to represent changes in sunlight-exposed spacecraft area, relative ballistic coefficients of the two spacecraft and fluctuations of the solar radiation intensity itself. The dead-band requirement for SPECS are still not well known, but a somewhat strict, and therefore conservative, estimate is about $6 \mu \mathrm{m}$ (estimated from information in [Leisawitz, 2000]). When translated into the single dimension of the simulation the dead-band becomes about 3.5 $\mu \mathrm{m}$. The tethered geometry and the continual rotation of the connected formation reduce the amount of responsibility for formation maintenance that falls on the propulsion system. Therefore, 12 thrusters are expected to be suitable to keep the apertures in the plane of the main truss structure and in the correct azimuthal position about the constellation's line of sight while providing some redundancy.

The $\Delta \mathrm{v}$ budget for the SPECS mission is $136.2 \mathrm{~m} / \mathrm{s}$ for each mirror. This simply comes from the total impulse imparted by the average solar radiation disturbance force multiplied by the expected mission lifetime of 12 years and then divided by the estimated aperture mass of $125 \mathrm{~kg}$.

\subsubsection{PPT Design}

TABLE 5.17 SPECS Pulsed Plasma Thruster Design Specifications

\begin{tabular}{|c|c|}
\hline Design Variable & Value \\
\hline \hline $\mathrm{I}_{\mathrm{bit}}[\mu \mathrm{Ns}]$ & 64 \\
$\mathrm{E}_{\mathrm{o}}[\mathrm{J}]$ & 8.88 \\
$\mathrm{PRF}_{\max }[\mathrm{Hz}]$ & 4 \\
$\mathrm{P}_{\mathrm{norm}}[\mathrm{W}]$ & 0.89 \\
$\mathrm{P}_{\max }[\mathrm{W}]$ & 35.52 \\
$\mathrm{I}_{\mathrm{sp}}[\mathrm{s}]$ & 1286 \\
$\mathrm{~m}_{\text {shot }}[\mu \mathrm{g}]$ & 5.08 \\
$\mathrm{I}_{\max }[\mathrm{A}]$ & 1.49 \\
$\mathrm{~m}_{\text {cap }}[\mathrm{kg}]$ & 0.1 \\
$\mathrm{~m}_{\mathrm{ppu}}[\mathrm{kg}]$ & 0.68 \\
\hline
\end{tabular}


TA BLE 5.17 SPECS Pulsed Plasma Thruster Design Specifications

\begin{tabular}{|c|c|}
\hline Design Variable & Value \\
\hline \hline $\mathrm{m}_{\mathrm{dic}}[\mathrm{kg}]$ & 0.23 \\
$\mathrm{~m}_{\mathrm{s}}[\mathrm{kg}]$ & 0.5 \\
$\mathrm{~m}_{\mathrm{prop}}[\mathrm{kg}]$ & 3.13 \\
$\mathrm{~m}_{\text {total } / \mathrm{sc}}[\mathrm{kg}]$ & 17.13 \\
$\mathrm{~m}_{\text {total }}[\mathrm{kg}]$ & 51.39 \\
\hline
\end{tabular}

\subsubsection{Colloid Thruster Design}

TABLE 5.18 SPECS Colloid Thruster Design Specifications

\begin{tabular}{|c|c|}
\hline Design Variable & Value \\
\hline \hline $\mathrm{F}_{\mathrm{N}}[\mu \mathrm{N}]$ & 0.13 \\
$\mathrm{I}_{\mathrm{N}}[\mathrm{nA}]$ & 123 \\
$\mathrm{~V}_{\text {nom }}[\mathrm{V}]$ & 2000 \\
$\mathrm{~V}_{\text {norm }}[\mathrm{V}]$ & 3500 \\
$\mathrm{~V}_{\max }[\mathrm{V}]$ & 5000 \\
$\mathrm{~F}_{\max }[\mu \mathrm{N}]$ & 100 \\
$\mathrm{~N}[]$ & 487 \\
$\mathrm{I}_{\text {sp norm }}[\mathrm{s}]$ & 509 \\
$\mathrm{I}_{\text {norm }}[\mu \mathrm{A}]$ & 32.1 \\
$\mathrm{I}_{\text {max }}[\mu \mathrm{A}]$ & 59.7 \\
$\mathrm{P}_{\text {norm }}[\mathrm{W}]$ & 0.15 \\
$\mathrm{P}_{\max }[\mathrm{W}]$ & 0.4 \\
$\mathrm{P}_{\text {neutral norm }}[\mathrm{mW}]$ & 16.1 \\
$\mathrm{P}_{\text {neutral max }}[\mathrm{mW}]$ & 29.9 \\
$\mathrm{~m}_{\text {prop }}[\mathrm{kg}]$ & 3.37 \\
$\mathrm{~m}_{\mathrm{s}}[\mathrm{kg}]$ & 0.33 \\
$\mathrm{~m}_{\text {ppu }}[\mathrm{kg}]$ & 0.14 \\
$\mathrm{~m}_{\text {feed }}[\mathrm{kg}]$ & 4.44 \\
$\mathrm{~m}_{\text {neutral }}[\mathrm{kg}]$ & 0.03 \\
$\mathrm{P}_{\text {total norm }}[\mathrm{W}]$ & 0.17 \\
$\mathrm{P}_{\text {total max }}[\mathrm{W}]$ & 0.43 \\
$\mathrm{~m}_{\text {total } / \mathrm{sc}}[\mathrm{kg}]$ & 9.65 \\
\hline &
\end{tabular}


TABLE 5.18 SPECS Colloid Thruster Design Specifications

\begin{tabular}{|c|c|}
\hline Design Variable & Value \\
\hline \hline $\mathrm{m}_{\text {total }}[\mathrm{kg}]$ & 28.95 \\
\hline
\end{tabular}

\subsubsection{FEEP Thruster Design}

TABLE 5.19 SPECS FEEP System Design Specifications

\begin{tabular}{|c|c|}
\hline Design Variable & Value \\
\hline \hline $\mathrm{F}_{\text {nom }}[\mu \mathrm{N}]$ & 25 \\
$\mathrm{~F}_{\text {max }}[\mu \mathrm{N}]$ & 50 \\
$\mathrm{~V}_{\mathrm{e} \text { nom }}[\mathrm{V}]$ & 3567 \\
$\mathrm{~V}_{\mathrm{e} \mathrm{norm}}[\mathrm{V}]$ & 4044 \\
$\mathrm{~V}_{\mathrm{e} \max }[\mathrm{V}]$ & 4130 \\
$\mathrm{~V}_{\mathrm{a}}[\mathrm{V}]$ & -3000 \\
$\mathrm{I}_{\mathrm{sp}}[\mathrm{s}]$ & 7778 \\
$\mathrm{I}_{\mathrm{e} \text { norm }}[\mathrm{mA}]$ & 0.46 \\
$\mathrm{I}_{\mathrm{e} \mathrm{max}}[\mathrm{mA}]$ & 0.51 \\
$\mathrm{I}_{\mathrm{a} \text { norm }}[\mu \mathrm{A}]$ & 92.7 \\
$\mathrm{I}_{\mathrm{a} \text { max }}[\mathrm{mA}]$ & 0.10 \\
$\mathrm{P}_{\text {in norm }}[\mathrm{W}]$ & 2.15 \\
$\mathrm{P}_{\text {in max }}[\mathrm{W}]$ & 2.4 \\
$\mathrm{P}_{\text {neutral norm }}[\mathrm{W}]$ & 0.23 \\
$\mathrm{P}_{\text {neutral max }}[\mathrm{W}]$ & 0.25 \\
$\mathrm{~m}_{\mathrm{s}}[\mathrm{kg}]$ & 0.46 \\
$\mathrm{~m}_{\mathrm{ppu}}[\mathrm{kg}]$ & 0.99 \\
$\mathrm{~m}_{\text {neutral }}[\mathrm{kg}]$ & 0.03 \\
$\mathrm{~m}_{\text {prop }}[\mathrm{kg}]$ & 0.22 \\
$\mathrm{P}_{\text {total norm }}[\mathrm{W}]$ & 2.39 \\
$\mathrm{P}_{\text {total max }}[\mathrm{W}]$ & 2.67 \\
$\mathrm{~m}_{\text {total } / \mathrm{sc}}[\mathrm{kg}]$ & 12.03 \\
$\mathrm{~m}_{\text {total }}[\mathrm{kg}]$ & 36.09 \\
\hline
\end{tabular}




\subsubsection{Cold Gas Thruster Design}

TABLE 5.20 SPECS Cold Gas Thruster Design Specifications

\begin{tabular}{|c|c|}
\hline Design Variable & Value \\
\hline \hline $\mathrm{I}_{\text {bit }}[\mu \mathrm{Ns}]$ & 120 \\
$\mathrm{~F}[\mathrm{mN}]$ & 12 \\
$\mathrm{w}_{\mathrm{p}}[\mathrm{s}]$ & 0.01 \\
$\mathrm{I}_{\mathrm{sp}}[\mathrm{s}]$ & 65 \\
$\mathrm{P}_{\text {valve }}[\mathrm{W}]$ & 10 \\
Avg $\mathrm{P}_{\text {valve }}[\mathrm{mW}]$ & 3.8 \\
$\mathrm{~m}_{\text {prop }}[\mathrm{kg}]$ & 192.5 \\
$\mathrm{~m}_{\text {feed }}[\mathrm{kg}]$ & 60 \\
$\mathrm{~m}_{\mathrm{s}}[\mathrm{g}]$ & 0.01 \\
$\mathrm{~m}_{\text {total } / \mathrm{sc}}[\mathrm{kg}]$ & 252.64 \\
$\mathrm{~m}_{\text {total }}[\mathrm{kg}]$ & 757.92 \\
\hline
\end{tabular}




\section{Chapter 6}

\section{PROPULSION SYSTEM SELECTION RESULTS}

\subsection{ST-3}

\subsubsection{Propulsion System Selection}

In order to clarify the system selection process, the somewhat raw design data presented in the previous chapter have been reduced to a list of parameters that directly affect the selection metrics discussed in Chapter 3. The parameters are defined as follows. The fixed mass is the mass of all of the propulsion system associated hardware that does not change when the mission lifetime is lengthened or shortened. This includes thruster structure, power processing units and other hardware that varies depending on the particular propulsion system. The variable mass consists of the propellant mass and the propellant feed system mass (if one is necessary for the thruster), which changes with mission lifetime because of the amount of propellant it must store and distribute. The mass fraction is just the sum of the fixed and variable masses divided by the total budgeted spacecraft mass. For the ST-3 mission the mass fraction is the total mass of the propulsion systems on each of the two spacecraft divided by the sum of the masses of the two spacecraft. Following the mass figures, two power estimates (normal and maximum usage, taken directly from the tables in Chapter 5) and their mass equivalents are shown. The mass equivalents are based on the estimated mass of solar arrays or secondary batteries necessary to provide the power. Based on estimates from Space Mission Analysis and Design [Larson, 1992], solar arrays have a mass specific power of approximately $100 \mathrm{~W} / \mathrm{kg}$, so the equivalent mass is 
simply the power draw divided by 100 . The relationship for the mass of the required batteries is slightly more complicated. The energy density of a nickel-hydrogen battery is estimated at $2.16 \times 10^{5} \mathrm{~J} / \mathrm{kg}$. However, the depth of discharge (DOD) is restricted to approximately $50 \%$ in order for them to last for a mission lifetime. In addition, two batteries are necessary to provide redundancy and to supply the required power to two thrusters at a time in the event that it is necessary. Therefore, battery mass incurred by a high power demand, $P_{\max }$, for a length of time, $t$, is given by

$$
\text { Equivalent Battery Mass }=2 \frac{P_{\max } t}{D O D\left(2.16 \times 10^{5}\right)}
$$

The fact that the propulsion systems for ST-3 are designed to perform both formation flying stationkeeping functions and formation reorientation functions makes the equivalent battery and solar array mass calculations slightly different from the other missions. In this case, normal formation flying thrusting power is provided strictly by solar arrays because in all cases the power draw of the thrusters is relatively small. However, for repositioning higher thrust levels are required for extended periods of time. Therefore, it is assumed that only half of the solar array power generation capacity can be allocated to the propulsion system during repositioning/reorientation. The total solar array power generated is approximated to be 250 Watts (provided by $1 \mathrm{~m} 2$ of illuminated Gallium-Arsenide photovoltaic cells). The only propulsion system requiring more than 125 Watts of power during peak usage is the FEEP system. As a result, it is the only propulsion system that requires an equivalent battery mass to be included in the system launch cost.

The next attribute listed in the tables is the Technology Readiness Level (TRL), based on the description as detailed in Table 3.1. Finally, the dead-band within which each propulsion system is capable of keeping the spacecraft is listed, as was determined from the simulation results that appear in Appendix B in the form of plots. The dead-band is used more as a requirement to be met than as a means for evaluating the performance of a system, but its impact on system performance will be described in 'Discussion of Trades' sec- 
tions. The values for the nine attributes are tabulated for each of the propulsion systems for the ST-3 mission in Table 6.1.

TABLE 6.1 ST-3 Propulsion System Table

\begin{tabular}{|c|cccc|}
\hline Metrics & PPT & Colloid & FEEP & Cold Gas \\
\hline \hline Fixed Mass [kg] & 33.17 & 32.76 & 52.78 & 0.24 \\
Variable Mass [kg] & 1.63 & 5.23 & 0.33 & 25.46 \\
Mass Fraction (\%) & 7.7 & 8.4 & 11.8 & 5.7 \\
Normal Power [W] & 0.5 & 0.0187 & 0.37 & 0.0053 \\
Maximum Power [W] & 76.2 & 11.5 & 239.6 & 15 \\
Solar Array Mass [kg] & 1.52 & 0.24 & 1.25 & 0.30 \\
Battery Mass [kg] & - & - & 26.74 & - \\
TRL & 7.5 & 3.5 & 5 & 6.5 \\
$\Delta \mathrm{x}[\mathrm{m}]$ & 0.01 & $\sim 1 \times 10^{-11}$ & $\sim 1 \times 10^{-12}$ & 0.01 \\
\hline
\end{tabular}

The last layer of data analysis involved in judging the propulsion systems and determining the best one for the mission is the cost analysis. As described in Chapter 3, costs are attributed to the technology research and development, the manufacture of the flight hardware and the launch of the complete system hardware. The costs associated with the mass, power and technology readiness for the ST-3 mission spacecraft are presented in Figure 6.1. The different patterns in the bars represent each of these three cost contributions. Each bar represents the composite cost of the propulsion system whose name appears below it. In the case of ST-3, it appears that the pulsed plasma thruster system is the lowest cost system, while the cold gas system is the next lowest cost. It can be seen that, based solely on the launch and hardware costs, the cold gas thruster system is less costly. This result is driven primarily by the small $\Delta v$ requirement (because of the short lifetime) of the mission. There is little penalty for the low specific impulse of the cold gas as a result and it has the least massive hardware. 


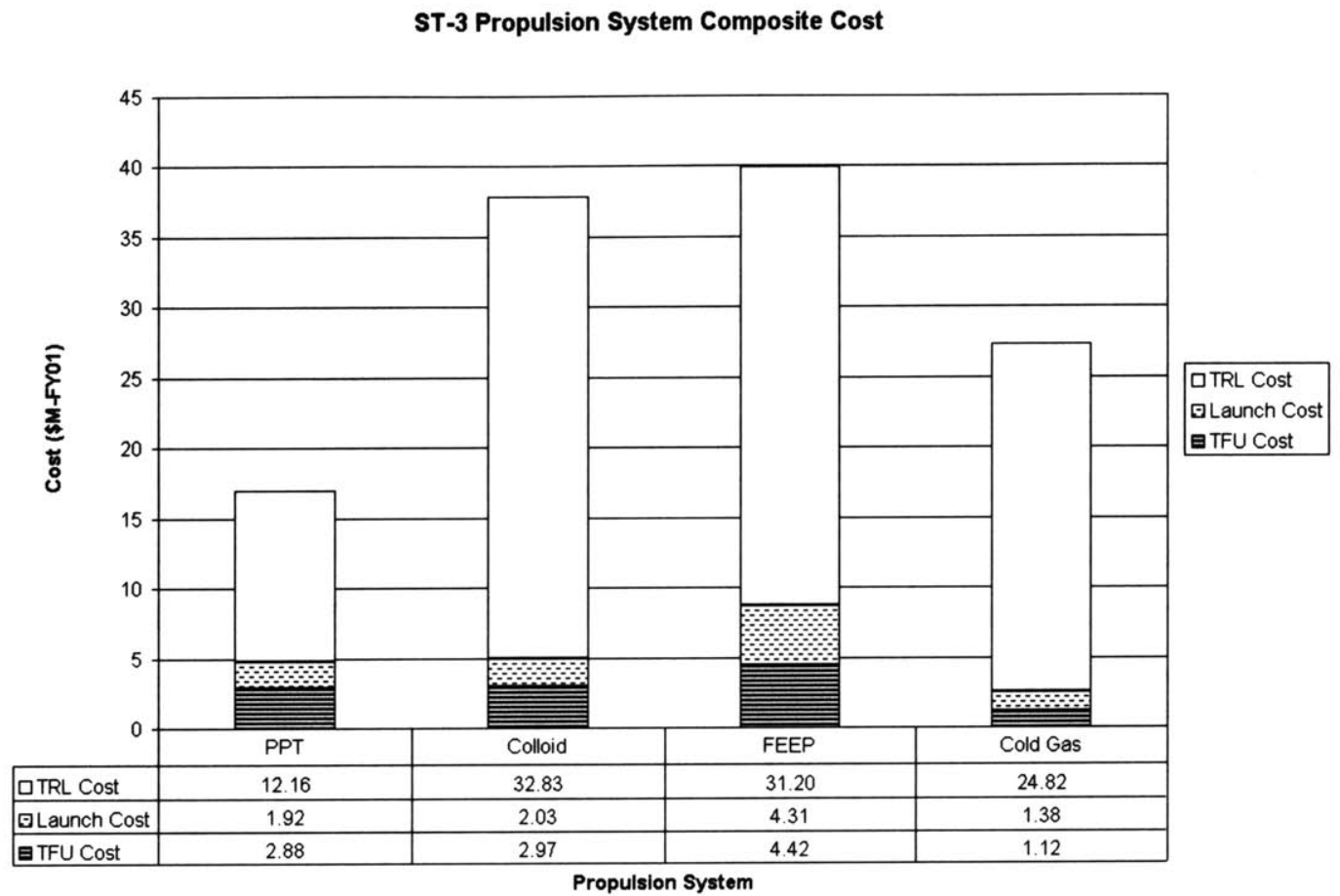

Figure 6.1 ST-3 propulsion system total costs including development, hardware and launch.

\subsubsection{Discussion of Trades}

With regard to the technology readiness levels, it is necessary to qualify the results. The TRL costs have a strong influence on the propulsion system selection and are, themselves, very sensitive to the TRL specifications, which can only be very approximately determined. Therefore, it is felt that it would be useful to view the selections from both a long term and short term perspective. The short term perspective is one in which the total costs, including the TRL cost, are the basis for judgement because the technology development would be the responsibility of the particular program managing the mission. Conversely, the long term perspective may ignore the TRL costs in deciding between the propulsion technologies because there is the chance and opportunity for the technologies to be developed at somewhat else's cost (at least partially) in the time between the present and the mission launch date. By these two lines of reasoning the PPT system should be 
chosen for the short term mission scenario and the cold gas system should be chosen in the longer term scenario.

There is another trade that was explicitly addressed previously for the ST-3 mission. For the ST-3 mission the precision formation flying enabling propulsion system is assumed to be the only propulsion system on the spacecraft. It must be capable of satisfying both the formation flying requirements and the spacecraft repositioning needs. As a result, the propulsion system must be rather versatile. When a larger view of the mission is taken, it is seen that various optical systems (potentially including optical delay lines, piezo-electric actuators, voice coils and fast steering mirrors) are needed to bring the error from the relative positioning of the spacecraft down to a level commensurate with the interferometric needs of the mission. There is the potential for eliminating some of these intermediate optical systems by using a propulsion system that can maintain a smaller dead-band [Grogan, 1998]. While the details of this trade are not studied in this work, it is noted that there may be the potential to save mass and/or cost by eliminating some of these optical systems and using a slightly more massive or more expensive propulsion system to maintain position more accurately. It is in this sense that the dead-band width listed in Table 6.1 may be used as a performance metric in some cases. From that table, it appears that the increased position accuracy gained over the cold gas and PPT systems by the colloid and FEEP thrusters may have the potential to streamline the optical delay system while maintaining the ability to perform repositioning maneuvers.

\subsection{TPF}

\subsubsection{Propulsion System Selection}

Table 6.2 is laid out for TPF exactly as Table 6.1 is for the ST-3 mission. The most notable difference between the two tables, aside from the differing values, is the absence of battery masses for all but the PPT system. This is because the propulsion system designs for TPF do not assume any of the major repositioning functions. They only handle position maintenance functions. The PPT system requires batteries because of the high repeti- 
tion rate needed to provide enough impulse via relatively small impulse bits (1200 $\mu \mathrm{Ns,}$ given the size of the TPF spacecraft, $800-850 \mathrm{~kg}$ ). While the FEEP system has a relatively high maximum power requirement, it cannot be provided by batteries because it is required continuously over an undetermined period of time.

TABLE 6.2 TPF Propulsion System Selection Table

\begin{tabular}{|c|cccc|}
\hline Metrics & PPT & Colloid & FEEP & Cold Gas \\
\hline \hline Fixed Mass [kg] & 216.2 & 48.03 & 99.65 & 0.8 \\
Variable Mass [kg] & 6.35 & 137.51 & 4.81 & 758.59 \\
Mass Fraction (\%) & 5.3 & 4.4 & 2.5 & 18.1 \\
Normal Power [W] & 15.15 & 0.77 & 16.64 & 0.22 \\
Maximum Power [W] & 347.07 & 2.59 & 52.84 & 15 \\
Solar Array Mass [kg] & - & 0.13 & 2.64 & 0.75 \\
Battery Mass [kg] & 1.33 & - & - & - \\
TRL & 7.5 & 3.5 & 5 & 7 \\
$\Delta \mathrm{x}[\mathrm{m}]$ & 0.01 & $\sim 1 \times 10^{-10}$ & $\sim 1 \times 10^{-10}$ & 0.01 \\
\hline
\end{tabular}

In the overall cost performance analysis for TPF, shown in Figure 6.2, the PPT system again performs the required functions for the minimum cost in the short term scenario, where the TRL cost is included. However, when viewed from the long term perspective, which may be more appropriate for TPF because of the estimated launch date (2011), the FEEP thruster system appears to be the best option. The long term perspective assumes that the system in question has been developed at another entity's cost by the time the mission is being designed and built. TPF has a long mission lifetime and with rather large reflective areas (because of the optics shielding) the disturbance force and therefore the overall impulse required for the mission is high, which favors high specific impulse systems. A cold gas thruster system is, therefore, categorically ruled out of consideration. For both ST-3 and TPF, the PPT systems have a considerable advantage in the short term perspective because similar systems have been successfully flown on past missions. The situation is likely to change for the missions requiring much smaller thrusts, on which no 
PPTs have yet been flown, and are only in the laboratory prototyping stage of development.

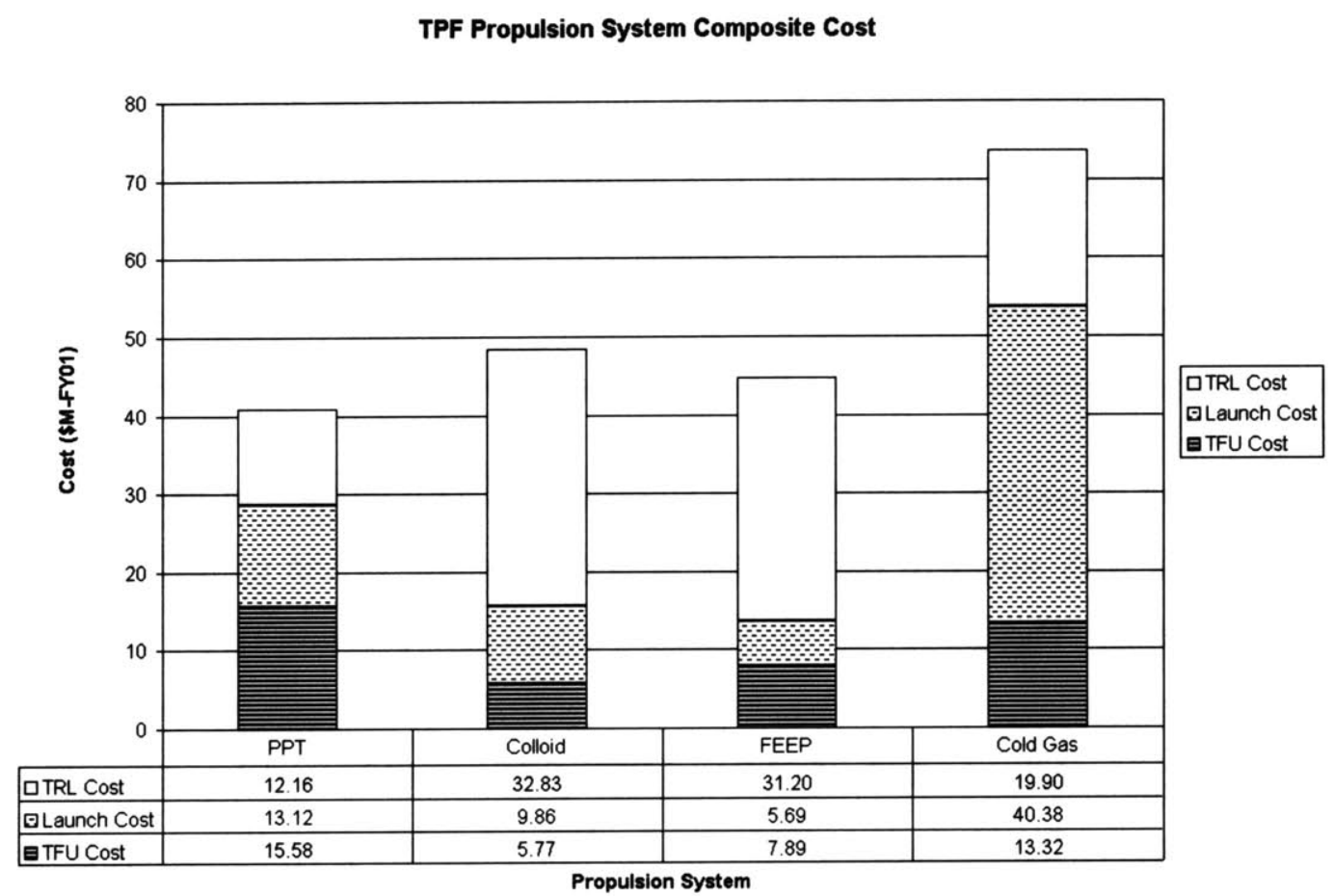

Figure 6.2 TPF propulsion system total costs including development, hardware and launch.

\subsubsection{Discussion of Trades}

From a long term perspective, the colloid thruster is not far behind because of its smaller hardware mass but significantly lower specific impulse. However, because the design presented for the colloid thruster requires very little power, it is conceivable that a colloid system operating with a higher accelerating voltage (and so, a higher specific impulse), or with a higher charge-to-mass ratio propellant (which would also increase the specific impulse) could be comparable or better suited to implementation in this mission than the FEEP system. 
The dead-band requirements for TPF are similar to those for ST-3 and therefore, relatively easy to meet for all of the propulsion systems considered here. The similarity to ST-3 also may suggest the possibility of designing only a single propulsion system for both the large translations and rotations of the cluster as well as the finer position and attitude maintenance maneuvering. However, this possibility was not adopted as the primary architecture for this study because of a lack of available detailed information concerning the $\Delta v$ and thrust requirements for that scenario. It is an idea worth exploring. Since that kind of architecture would place even more emphasis on the specific impulse of the propulsion system as a result of the increased $\Delta v$ needed, it is likely that the outcome of the study would continue to suggest (from the long term perspective) using a FEEP system .

Another strong consideration in the trade study for the TPF mission in particular, but in most missions with sensitive optics also, is contamination of the optical hardware by thruster effluents. Though it has been acknowledged as a pressing issue, there are currently few data available that could be used to aid in making a distinction between the systems. It is expected that both the FEEP and PPT plumes will have deleterious effects on the optical systems, but less is known about the effect of colloid propellant, in part because there are many different options (which may turn out to be a boon to colloid thrusters). While cold gas systems provide the least contamination, it is seen in Figure 6.2 that it is also the most costly, primarily because of its low specific impulse. In all cases, quantitative data that estimate the amount of degradation caused by propellant contamination are needed.

\subsection{LISA}

\subsubsection{Propulsion System Selection}

The LISA mission presents a somewhat different scenario than the two previously discussed missions, as has been mentioned before. Table 6.3 shows the most influential design parameters, but differs somewhat from the tables for TPF and ST-3. The battery mass equivalent is completely omitted because for all the thruster schemes (as can be seen 
in the simulation results in Appendix B) continuous thrusting is required. That is to say, that while the PPT and cold gas systems are pulsed, they must be fired at a fairly regular rate, which is rather high. Additionally, there are no long periods during which firing is not necessary because of the stringent stationkeeping requirements. These attributes prevent the effective and/or efficient use of secondary batteries. An additional row is included at the bottom of the table listing the maximum magnitude of the power spectral density of the position signal of the spacecraft at $0.1 \mathrm{~Hz}$ in order to indicate the system's compliance with the requirements, as noted in Chapter 2 . The numbers that appear in parentheses in the 'Variable Mass' and 'Mass Fraction' rows represent those values for the 10 year mission duration, while the other numbers in those rows are for the 2 year mission. All the other numbers do not change when the lifetime changes. This is the same notation convention used in the propulsion system design tables in Chapter 5.

TABLE 6.3 LISA Propulsion System Selection Table

\begin{tabular}{|c|cccc|}
\hline Metrics & PPT & Colloid & FEEP & Cold Gas \\
\hline \hline Fixed Mass [kg] & 26.07 & 20.82 & 46.97 & 0.48 \\
Variable Mass [kg] & $1.65(8.22)$ & $1.8(4.23)$ & $0.036(0.186)$ & $6.51(32.07)$ \\
Mass Fraction (\%) & $4.6(5.7)$ & $3.8(4.2)$ & $7.8(7.9)$ & $1.2(5.4)$ \\
Normal Power [W] & 24 & 0.062 & 0.84 & 0.58 \\
Maximum Power [W] & 40.8 & 0.43 & 5.55 & 10 \\
Solar Array Mass [kg] & 1.22 & 0.013 & 0.17 & 0.3 \\
TRL & 3.5 & 3.5 & 5.5 & 2.5 \\
$\Delta \mathrm{x}$ & $1 \times 10^{-9}$ & $\sim 4 \times 10^{-12}$ & $\sim 1 \times 10^{-13}$ & $1 \times 10^{-9}$ \\
$\operatorname{PSD}_{\mathrm{x}}\left[\mathrm{m}^{2} \mathrm{~Hz}^{-1}\right] @ 0.1 \mathrm{~Hz}$ & $1 \times 10^{-19}$ & $5 \times 10^{-22}$ & $8 \times 10^{-26}$ & $5 \times 10^{-18}$ \\
\hline
\end{tabular}

Figure 6.3 shows the results of the cost analysis for the LISA mission, with the nominal two year duration. The disturbance forces that the spacecraft needs to counteract are small (in the tens of micronewtons) and with a mission duration of only 2 years the $\Delta \mathrm{v}$ requirements are minimal. This means that, like ST-3, specific impulse does not drive the propulsion system selection. This is obvious from the small variable masses listed in Table 6.3 for the nominal mission. This favors propulsion systems with low hardware masses. The 
cold gas system requires the least hardware because it does not consume much power, does not require a power processing unit and the required nozzles and valves must be very small in order to achieve the kind of impulse required. It should also be noted that while PPTs often have a relatively low hardware mass, they suffer from a higher power requirement than any of the other systems for this mission. The LISA spacecraft has a limited surface area on which to mount solar panels, so just incorporating a mass penalty for using more power may not capture the whole effect of having a strict power limit. However, as noted above, the PPT and cold gas systems may be problematic in terms of even having the ability to meet the mission requirements.

While the technology development rating always plays a large role in the short term perspective because all of the propulsion systems require significant refinement, the total costs in this scenario show that the TRLs have a leveling effect on the different systems. The chart shows that the difference in cost between the four propulsion systems is at most about two and a half million dollars, significantly less than the range of costs estimated for any of the other missions. This may very well be within the error bounds of these estimates, making it impossible to say which system will actually be the most appropriate.

In the case of the ten year mission duration, Figure 6.4 is somewhat different from the bar charts for the two year mission. The differences arise only as a result of the added propellant required to operate for five times as long. As would be expected, the FEEP system gains a small bit of ground, bringing the cost slightly closer to the colloid thruster. Again however, the difference in the cost between all four systems is small and the difference between the cold gas and the colloid system is only about $\$ 270,000$, while the FEEP system is only about $\$ 400,000$ more than the colloid thruster system. As a result, these costs alone do not give an accurate means for making a propulsion system selection for the LISA mission. Although, when the TRL costs are ignored, as is suggested for the long term perspective, and the performance issues with the cold gas and PPT systems are considered (see the discussion in Section 6.3.2 below) the colloid system costs about $\$ 3.29$ million less than the FEEP system. 
LISA (2 year) Propulsion System Composite Cost

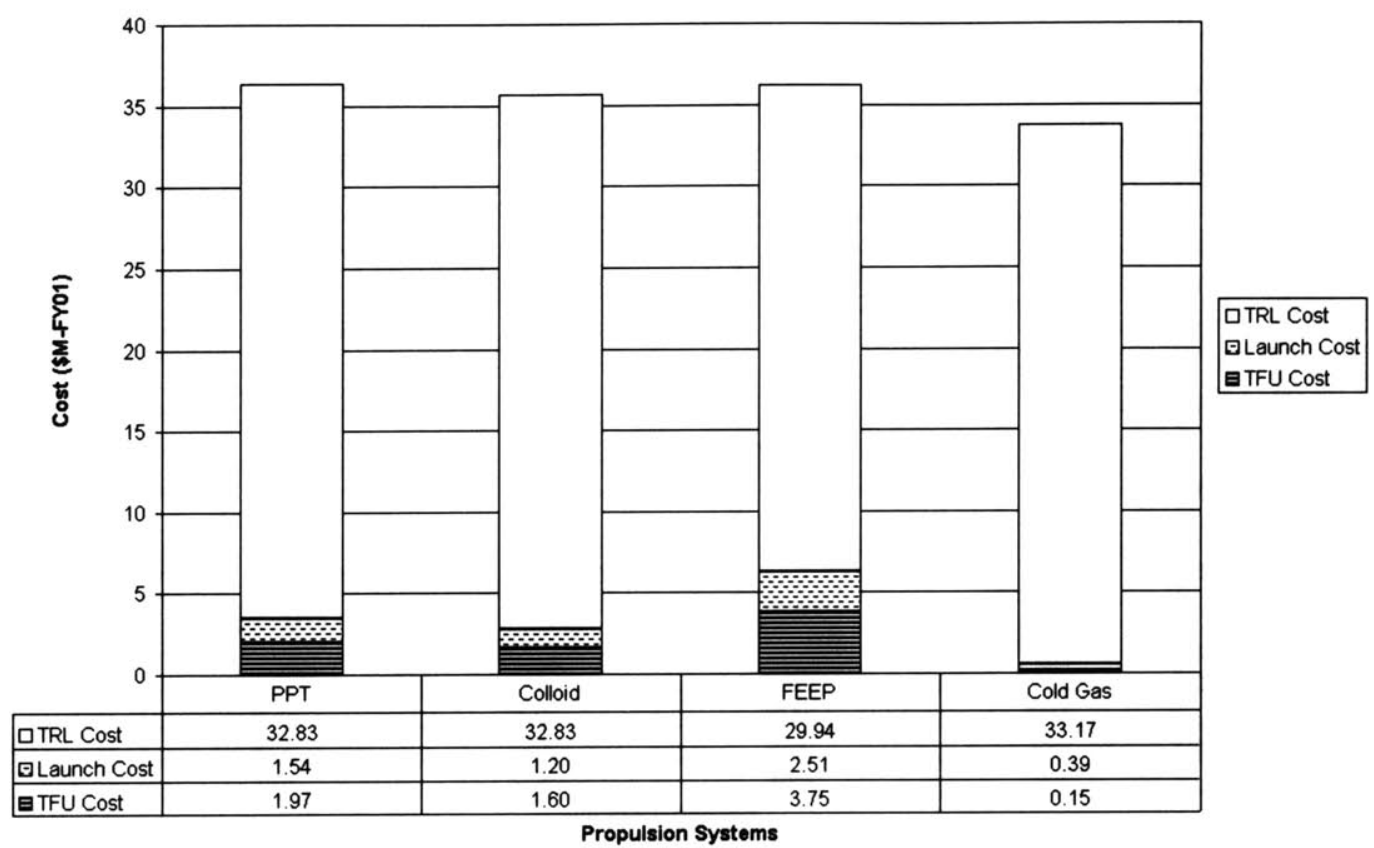

Figure 6.3 LISA (2 years) propulsion system total costs including development, hardware and launch.

\subsubsection{Discussion of Trades}

There are different issues to be considered in discussing the trades involved in selecting a propulsion system for LISA, as opposed to the previous two missions. The specification of the position maintenance control requirements in the form of spectral densities is a particularly important difference. Based on the requirements of the mission, there can be preliminary narrowing down of propulsion systems. The position requirements stated for LISA require the position to remain within $1 \mathrm{~nm} / \mathrm{Hz}^{1 / 2}$, which is squared to give the same units as the position power spectral density, resulting in a requirement of $\mathrm{PSD}_{\mathrm{x}}<1 \times 10^{-18}$ $\mathrm{m}^{2} / \mathrm{Hz}$. Table 6.3 shows that the cold gas thruster does not quite meet this requirement. Figure 6.5 shows the position power spectral density from the cold gas thruster LISA simulation after post processing has been done to incorporate a twice cascaded high pass filter with a bandwidth of $0.1 \mathrm{~Hz}$. This post processing is meant to represent the incorporation of a more sophisticated control algorithm than was originally used in the simulation so as 
LISA (10 year) Propulsion System Composite Cost

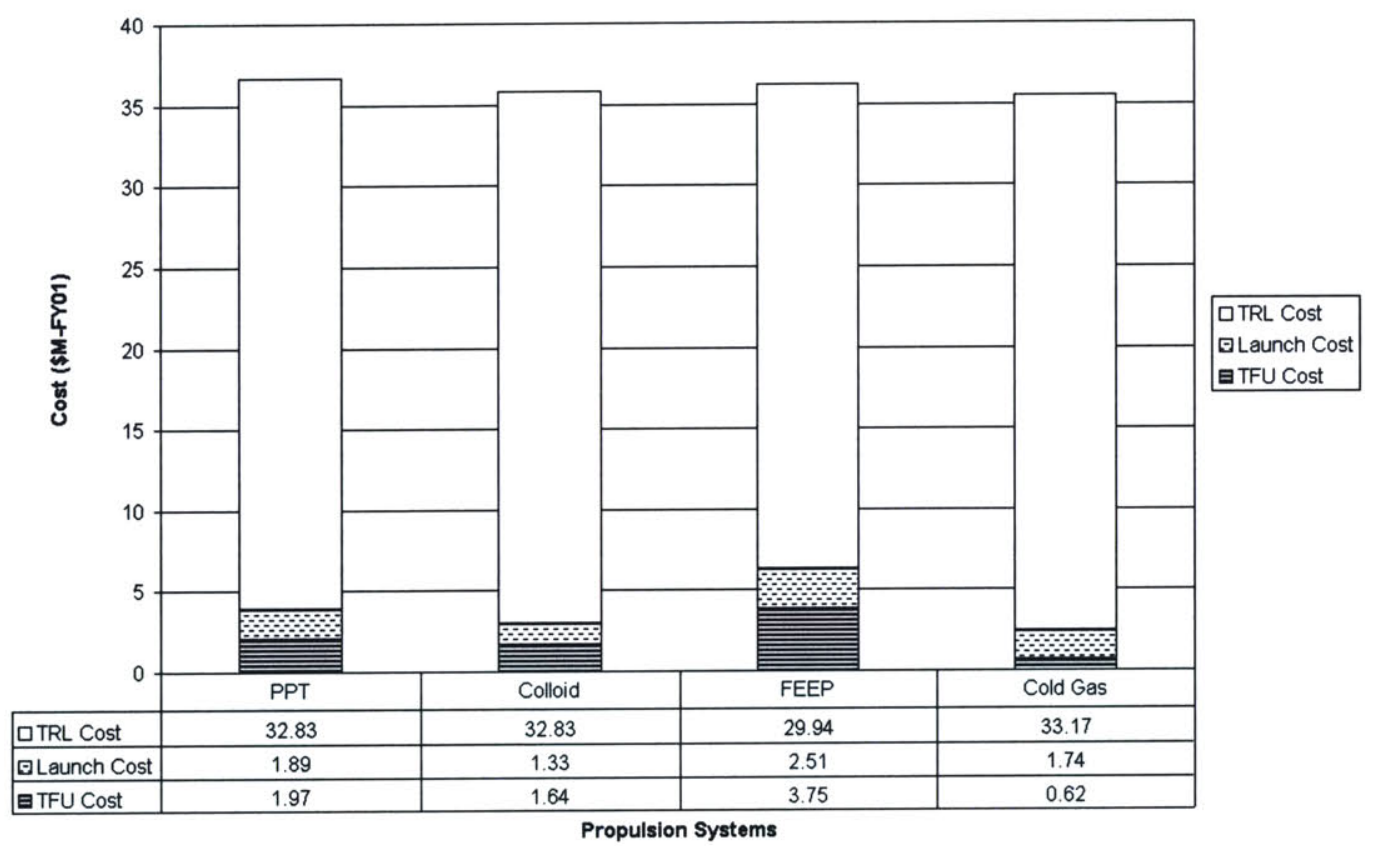

Figure 6.4 LISA (10 years) propulsion system total costs including development, hardware and launch.

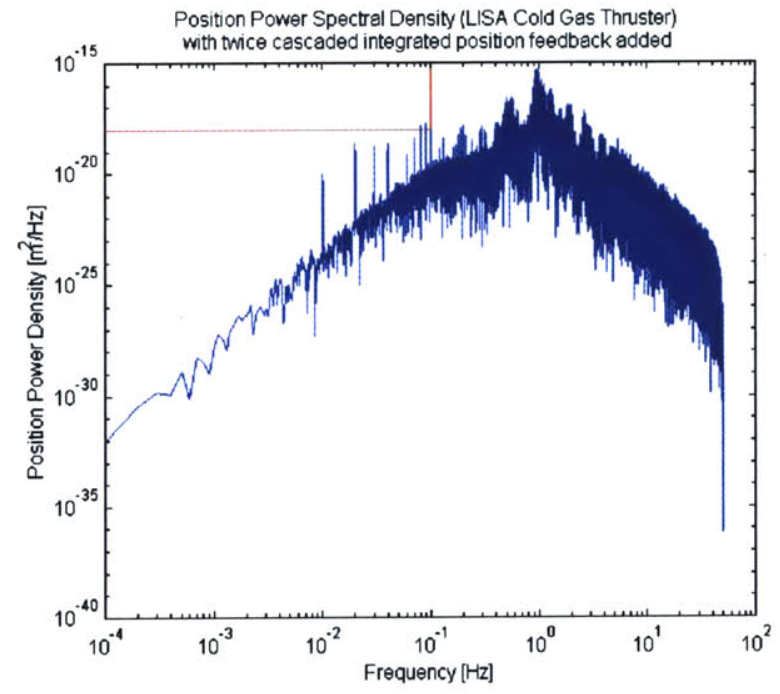

Figure 6.5 Power spectral density of position (cold gas thruster) after high pass filtering with requirement from LISA indicated by the red line 
to specifically eliminate low frequency drifts. The use of the high pass filter essentially corresponds to implementing regulation control on the integral of the position (in addition to regulating on position and velocity, which are included in the original simulation). While it can be seen that the cold gas thruster comes very close to meeting the requirement, there is uncertainty in the position of the spikes that break the requirement because they may depend on factors such as the disturbance force and its spectrum. Therefore, taking a conservative view because of the potential for large uncertainty, it is the opinion of the author that both the PPT (because its spectrum is very similar to the cold gas spectrum in the simulations for the LISA mission) and the cold gas thruster systems cannot reliably be said to meet the LISA mission requirements. Table 6.3 shows that both the colloid and FEEP thrusters have much larger margins in terms of the position PSD requirement, with the FEEP outperforming the colloid by approximately four orders of magnitude (based on the data from [Tajmar, 2000], not from simulation). Figure 6.6 shows the position PSD for the colloid thruster as was created from simulation data and from data provided by Manuel Gamero and the Busek Co. Inc.[Gamero, 2000], after the same post-processing scheme, as was described above, was implemented. While the col-

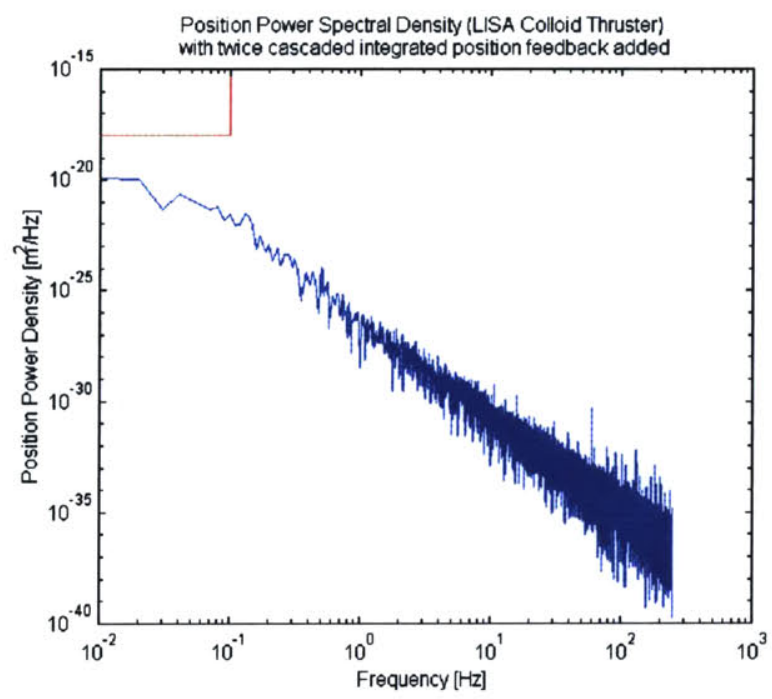

Figure 6.6 Power spectral density of position (colloid thruster) after high pass filtering with requirement from LISA indicated by the red line 
loid thruster position PSD does come within two orders of magnitude of the requirement at $0.01 \mathrm{~Hz}$ (data below that frequency is not available as yet), it is believed that the more important frequency band is nearer to $0.1 \mathrm{~Hz}$ because the effect of a high pass filter decreases as the frequency increases. The filter has a lesser ability to attenuate the signal in that band, so it is more important that the original signal be smaller there.

Another potential problem with using the cold gas thruster, particularly for the longer duration mission, is the possibility of significantly shifting the center of mass of the spacecraft during the mission by depleting the propellant tank. The other systems with higher specific impulses will suffer less from this problem. If there is no reliable way to track the movement of the center of mass, due to either propellant slosh and depletion, of the spacecraft, a mission in which the position and attitude need to be maintained very precisely may not be realizable.

\subsection{MAXIM}

\subsubsection{Propulsion System Selection}

For MAXIM, identical propulsion systems of some kind are required for 33 collector spacecraft, while a separate propulsion system is necessary for a single additional detector spacecraft. However, part of the propulsion system on the detector spacecraft (the part responsible for motion in a plane parallel to the plane of the ecliptic) has the same constraints and requirements as for the other 33 spacecraft. So, in Table 6.4 there are two columns for all of the parameters for each propulsion system, except for TRL and dead-band in a couple of cases. The first column under each propulsion system name is for the 33 collector spacecraft and the second column is for the single detector spacecraft, which explains the much higher mass figures in the first columns.

The cost analyses for MAXIM have been separated into two separate figures because of the dichotomy between the to different spacecraft involved. The first one, Figure 6.7, is for the set of collector spacecraft. The preferred propulsion system in this case appears to 
TABLE 6.4 MAXIM Propulsion System Selection Table

\begin{tabular}{|c|c|c|c|c|c|c|c|c|}
\hline Metrics & \multicolumn{2}{|c|}{ PPT } & \multicolumn{2}{|c|}{ Colloid } & \multicolumn{2}{|c|}{ FEEP } & \multicolumn{2}{|c|}{ Cold Gas } \\
\hline Fixed Mass [kg] & 138.4 & 4.48 & 105.07 & 2.53 & 255.98 & 5.94 & 2.64 & 0.06 \\
\hline Variable Mass [kg] & 0.53 & 1.16 & 14.19 & 11.29 & 0.1 & 0.36 & 8.58 & 63.53 \\
\hline Mass Fraction (\%) & 0.42 & 0.56 & 0.36 & 1.38 & 0.78 & 0.63 & 0.03 & 6.36 \\
\hline Normal Power [W] & 1.24 & 23.9 & 0.006 & 0.505 & 0.073 & 11.39 & 0.012 & 0.133 \\
\hline Maximum Power [W] & 20.67 & 39.83 & 0.017 & 0.86 & 0.2 & 11.52 & 10 & 10 \\
\hline Solar Array Mass [kg] & 6.82 & 0.398 & 0.006 & 0.009 & 0.066 & 0.152 & 3.3 & 0.1 \\
\hline TRL & 3.5 & 7 & \multicolumn{2}{|c|}{3.5} & \multicolumn{2}{|c|}{5.5} & 2.5 & 6.5 \\
\hline$\Delta \mathrm{x}[\mathrm{m}]$ & \multicolumn{2}{|c|}{$20 \times 10^{-9}$} & $5 \times 10^{-14}$ & $5 \times 10^{-12}$ & $5 \times 10^{-13}$ & $1 \times 10^{-11}$ & \multicolumn{2}{|c|}{$20 \times 10^{-9}$} \\
\hline
\end{tabular}

be cold gas from both the short term and long term perspectives. Again, as mentioned for other studies, this result relies highly on low $\Delta \mathrm{v}$ mission requirement. It should also be acknowledged that the cold gas thruster design for the MAXIM collector spacecraft requires a $12 \mu \mathrm{Ns}$ impulse bit, which is an order of magnitude smaller than any of the cold gas thruster systems currently in development. While the technology readiness level rating is supposed to account for these kinds of issues, there is a lot of room for error, especially in this case. While the other technologies have at least demonstrated, in some capacity, the ability to produce the appropriate thrust levels, a cold gas system has not yet been shown to be capable of this.

The MAXIM detector spacecraft propulsion system cost results are markedly different from that of the collector spacecraft. The detector spacecraft is much farther from the plane of the ecliptic and so must fight much larger perturbations than the collector spacecraft, resulting in a much larger $\Delta \mathrm{v}$ requirement. With fewer thrusters than most of the other missions, owing to the planned use of solar sails as mentioned before, the fixed propulsion system mass becomes a relatively small issue. As a result, when taking the long term development perspective the FEEP and colloid thruster systems are seen to be much more favorable (slightly more favorable in the case of the FEEP) than the cold gas system and slightly more cost effective than the PPT. However, in the short term, taking research 


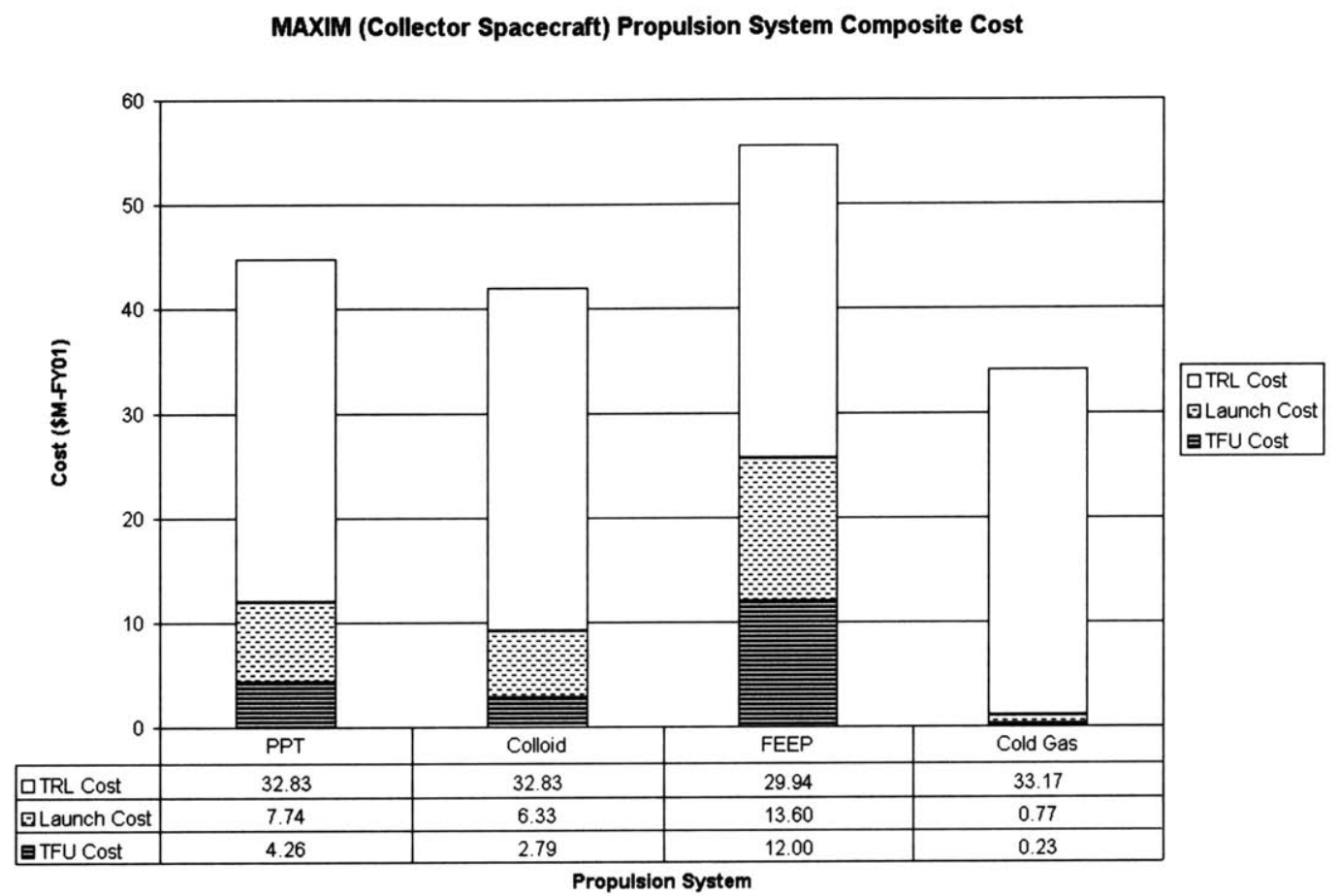

Figure 6.7 MAXIM (collector spacecraft) propulsion system total costs including development, hardware and launch.

and development costs into account, the PPT dominates the field because of its considerable flight heritage.

\subsubsection{Discussion of Trades}

For the architecture assumed in this study [Cash, 2000], most of the solar radiation induced perturbations to the spacecraft are to be handled by solar sail technology. As a result, the $\Delta \mathrm{v}$ required of the supplementary propulsion system, primarily for taking care of perturbations perpendicular to the plane of the ecliptic but also for trimming the work done by the solar sails, is very small. This study does not attempt to model or incorporate solar sail technology into the system selection. It should be noted, however, that if the propulsion system is required to perform all the aspects of the position maintenance, supplanting the solar sails, the $\Delta \mathrm{v}$ necessary will increase significantly (highly dependent on 


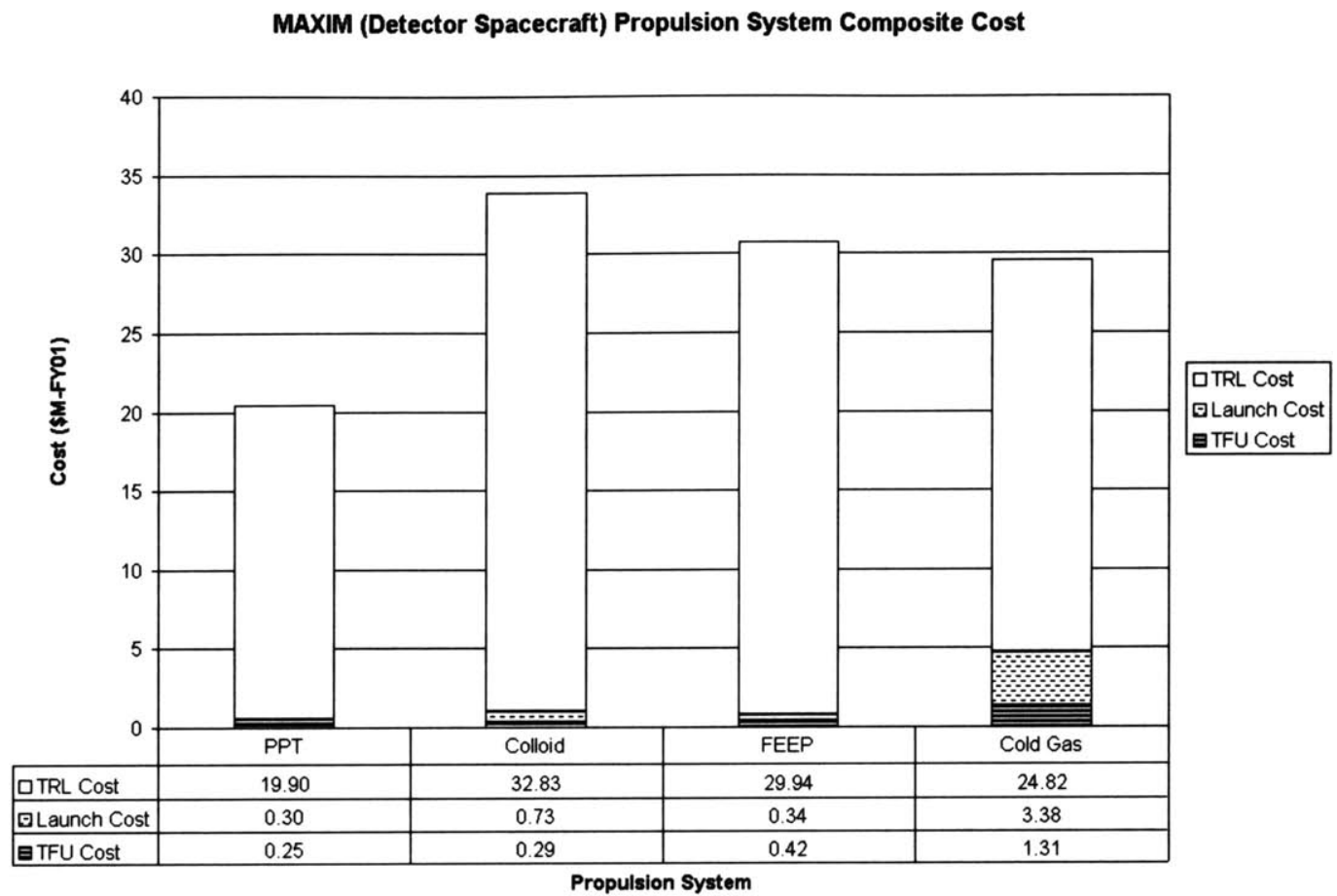

Figure 6.8 MAXIM (detector spacecraft) propulsion system total costs including development, hardware and launch.

spacecraft effective area). This may very well shift the selection to the next lowest cost system which is the colloid thruster.

Noteworthy as well, is the fact that the PPT systems for both MAXIM and LISA have rather low TRL levels, whereas several other PPT system designs are rated as more highly developed. This is because PPTs with the low impulse bits required for LISA and MAXIM are currently being developed and have no flight history or space environment tested hardware. If this technology is further developed for one of these missions or for a different mission, which seems rather likely given the progress that has been made so far, the short term attractiveness of the so called micro-PPT maybe increased enough by the time this mission is in the detailed design phase that it will be preferred. Similarly, the colloid and FEEP promise to be very cost effective options, for the detector spacecraft at least, if they are developed in the relatively near term for other missions. 


\subsection{SPECS}

\subsubsection{Propulsion System Selection}

The SPECS mission incorporates a rather small dead-band requirement with sizable $\Delta v$ requirements, while still being somewhat sensitive to fixed hardware mass. These attributes can be seen in Table 6.5. It shows that, like LISA and MAXIM, the dead-band size requires mainly continuous thrusting, requiring all power to be supplied by solar arrays. This is the cause for the relatively high power requirement shown for the PPT. It is fairly obvious that the cold gas system would be a very poor decision for the SPECS mission, despite its relatively high TRL, because the propulsion system mass would be more than twice as much as the budgeted total mass of the three spacecraft. This is mainly because of the poor specific impulse, which makes the variable mass extremely large.

TABLE 6.5 SPECS Propulsion System Selection Table

\begin{tabular}{|c|cccc|}
\hline Metrics & PPT & Colloid & FEEP & Cold Gas \\
\hline \hline Fixed Mass [kg] & 42 & 15.63 & 35.43 & 0.36 \\
Variable Mass [kg] & 9.39 & 13.32 & 0.66 & 757.56 \\
Mass Fraction (\%) & 13.7 & 7.7 & 9.6 & 202 \\
Normal Power [W] & 0.89 & 0.17 & 2.39 & 3.8 \\
Maximum Power [W] & 35.52 & 0.43 & 2.67 & 10 \\
Solar Array Mass [kg] & 1.07 & 0.013 & 0.081 & 0.3 \\
TRL & 7 & 3.5 & 5 & 6.5 \\
$\Delta \mathrm{x}[\mathrm{m}]$ & $5 \times 10^{-6}$ & $1 \times 10^{-12}$ & $1 \times 10^{-11}$ & $5 \times 10^{-6}$ \\
\hline
\end{tabular}

Based on a short term perspective, the PPT system costs less than the colloid and FEEP thrusters by approximately $\$ 10$ million, clearly a product of the advanced technological readiness of the PPT. From the long term perspective the message is a bit different. While the margins are not as large, the colloid thruster incurs a cost that is less than both the PPT and FEEP. The cold gas system, as anticipated, cannot compete with the other systems, given the requirements of this mission. Additionally, the FEEP and colloid thrusters have 
the ability to maintain a much tighter dead-band than the PPT, which could lead to further cost savings.

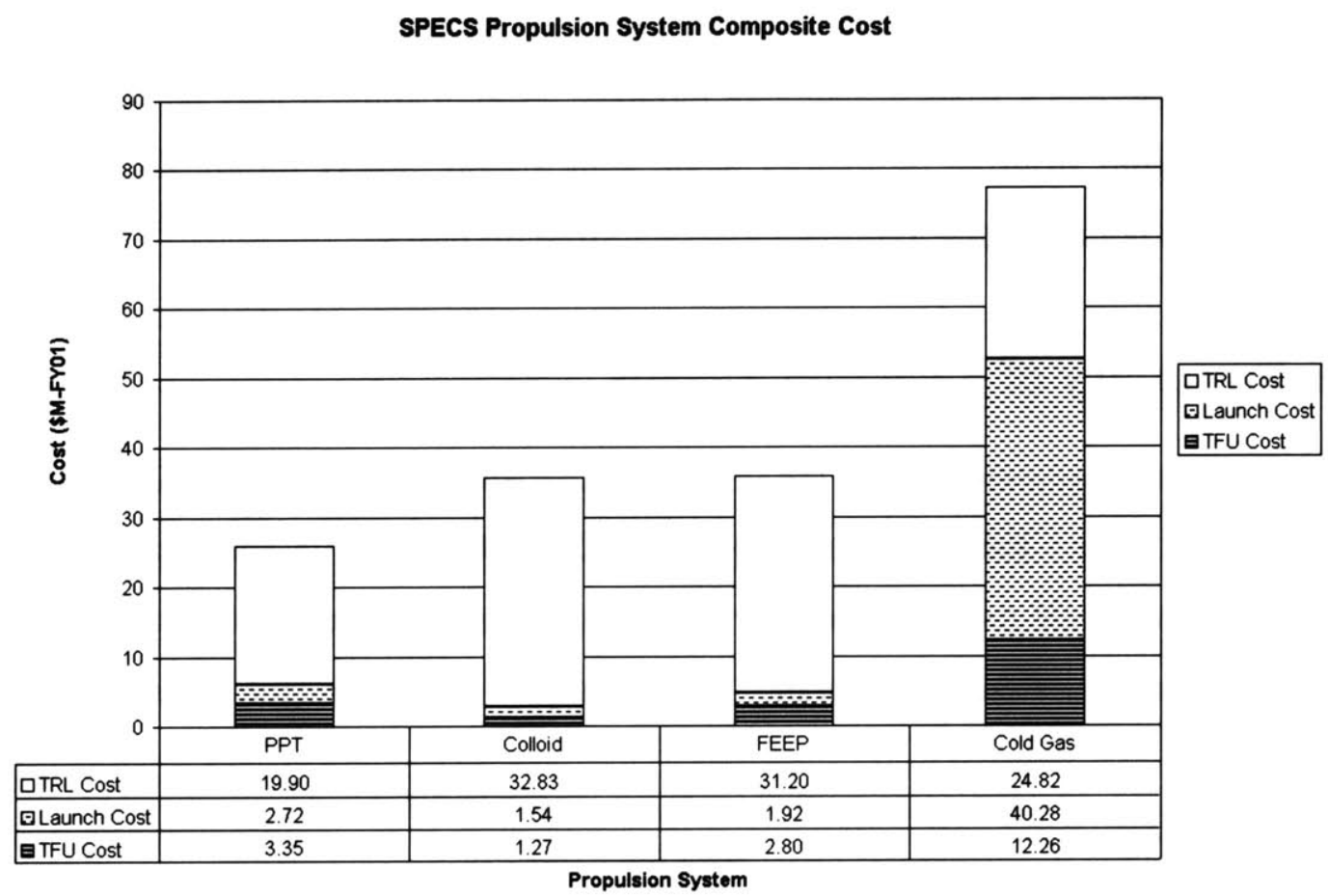

Figure 6.9 SPECS propulsion system total costs including development, hardware and launch.

\subsubsection{Discussion of Trades}

An issue similar to the optical system trade mentioned for the ST-3 mission may be applicable to the design of SPECS. That is, the optical interferometry system hardware mass may be able to be reduced by using a propulsion system that can maintain the spacecraft's positions more accurately than the specified requirement. In this case, it may be possible for the colloid and/or the FEEP systems to be preferable to the PPT even from the short term viewpoint and will certainly bolster the case for a colloid or FEEP thruster system when viewed from the long term perspective. 
Another potential issue with an impact on propulsion system selection is the use of structural tethers in this mission. There are certainly stability concerns with their implementation because of their similarity to pendulums. It is conceivable that a pulsed propulsion system could incite unstable oscillation modes. While the same can be said of continuous firing propulsion systems, much smaller thrust levels are necessary for continuous thrusting, resulting in much smaller swinging motions. Additionally, it may be easier to 'rescue' a spacecraft from an oscillatory mode with a more highly throttleable propulsion system that is not restricted to thrusting in quantized impulses.

\subsection{Summary of Selection Trends}

Figure 6.10 is a representation of the main characteristics of each of the missions in relation to each other. Each mission is positioned according to its spacecraft dead-band requirement, the total $\Delta v$ (summed over all the spacecraft in the mission) requirement and the mass of an individual spacecraft. A summary of the propulsion systems that were

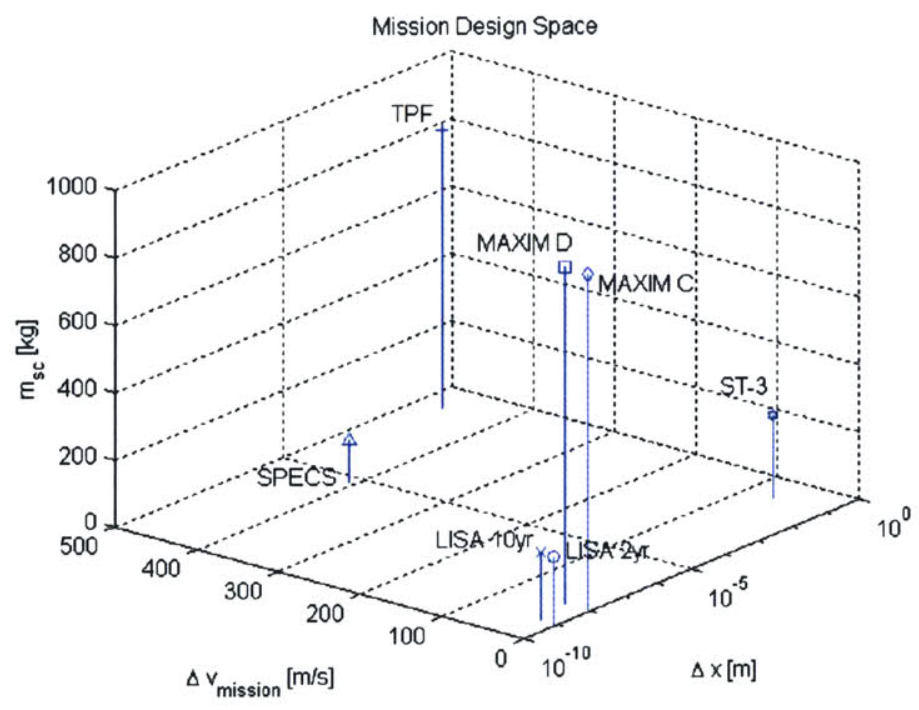

Figure 6.10 Three dimensional design (dead-band, $\Delta \mathrm{v}$, spacecraft mass) space containing the five missions. 
selected as the best choice for each mission, based on both the compiled cost estimates and the issues mentioned in the selection and trades discussions, are listed in Table 6.6. The

TABLE 6.6 Propulsion System Cost-Based Selection Summary

\begin{tabular}{|c|cccccc|}
\hline Perspective & ST-3 & TPF & LISA & \multicolumn{2}{c|}{ MAXIM } & SPECS \\
\hline \hline Short Term & PPT & PPT & $\begin{array}{c}\text { Colloid/ } \\
\text { FEEP }\end{array}$ & $\begin{array}{c}\text { Colloid/ } \\
\text { CG }\end{array}$ & PPT & PPT \\
Long Term & CG & FEEP & Colloid & $\begin{array}{c}\text { Colloid/ } \\
\text { CG }\end{array}$ & $\begin{array}{c}\text { FEEP/ } \\
\text { Colloid/ } \\
\text { PPT }\end{array}$ & $\begin{array}{c}\text { Colloid/ } \\
\text { FEEP }\end{array}$ \\
\hline
\end{tabular}

selections are provided for both the short term and long term perspectives. The short term perspective shows that the PPT system dominates the selections likely in large part due to the fact that is the most highly developed system with a high performance specific impulse. However, there are niches in the design space where each of the propulsion systems seems to be preferable. Additionally, there are a few cases in which there are several propulsion systems that can meet the mission requirements efficiently. Particularly, the similarity between the FEEP and colloid systems in cases where the $\Delta v$ requirements are not extremely large and there is not a large number of thruster units required. Regardless of the details, it does appear that both the colloid and FEEP thruster systems have applications in several of these precision formation flying missions and therefore warrant further development. 


\section{Chapter 7}

\section{CONCLUSIONS}

\subsection{Design Recommendations}

\subsubsection{Propulsion System Selection}

The selection of the precision formation flying propulsion systems for the five missions examined in this study is based on three major criteria. They include performance, cost and technical feasibility. Performance is judged by the ability to meet specified mission requirements. Cost is based on hardware manufacturing, launch and necessary research and development. While not included quantitatively, the technical feasibility of developing a propulsion technology is discussed and used to influence the recommendation of propulsion systems for implementation on the missions. It is shown that all of the propulsion systems examined have potential areas of usefulness.

Many of the results of the selection process follow intuitive and traditional trends, which adds confidence to some of the less intuitive results. For example, the general trend shows missions with high velocity increment requirements being best suited by propulsion systems with higher specific impulses. In some cases economy in fixed propulsion system hardware mass offsets the effect of specific impulse differences because of only modest $\Delta v$ requirements. 
Additionally, it is seen that the extent of technological development of a propulsion technology can have a large impact on the financial resources necessary for its implementation on a given mission. Therefore, there is some imperative to focus resources (primarily public/governmental) on propulsion systems that have the largest number of opportunities for implementation so that missions in the farther future will benefit from technology legacy. In the meantime private interests in need of similarly capable propulsion technologies will naturally opt for implementing propulsion systems that are more financially favorable in the shorter term.

\subsubsection{Future Propulsion System Development}

The results of this propulsion system study urge the further development of a pair of somewhat immature technologies. Both the colloid and FEEP thruster systems appear to capture niches in precision formation flying missions that are not as well performed by the other systems. The primary niche is one requiring very fine thrust controllability, particularly for smaller spacecraft with moderate to high $\Delta v$ requirements. On the other hand, the miniature cold gas thruster and micro-pulsed plasma thruster systems have few applications in the set of missions studied.

The miniature cold gas thruster system suffers first from its inherently low specific impulse. However, this is not a large problem unless the mission requires a large $\Delta v$ to be delivered. A larger potential problem is the ability to manufacture valves small and reliable enough to work in conjunction with the micro-machined nozzles necessary to provide extremely small thrust levels. While such nozzles have been produced in several cases, adequate valves and the other interfaces have not yet been created with the same performance and quality. One of the primary concerns in expending resources in further development is the issue of valve leakage in MEMS fabricated valves. If such valves are eventually successfully manufactured then there may be low $\Delta v$ precision formation flying missions that would benefit from the use of MEMS cold gas thruster systems. Otherwise, it appears that very small, but traditionally manufactured cold gas thruster systems 
should be used for somewhat less precise formation flying (as well as other more traditional functions) where a large $\Delta v$ is not necessary.

As for the micro-PPT, it does not suffer so much from low specific impulse as the miniature cold gas thruster, though early experiments show values only around 150 or so seconds. The micro-PPT has been demonstrated in a laboratory as a relatively complete breadboard system, though. These prototypes are small and simple and therefore appear to be somewhat more promising for actual future use than the miniature cold gas thrusters. Still, it is not quite obvious in what regime the micro-PPT's strengths lie. However, they have a low fixed mass, which may be valuable in missions requiring very high reliability (large amount of redundancy) without a large $\Delta v$ requirement. Another potential problem may be that, unlike the traditional PPT, the micro-sized version ablates its metal anode and cathode and probably emits larger quantities of metal ions as a result. This may have deleterious effects on spacecraft optics, as is a major concern for FEEP thrusters, which accelerate metal ions.

\subsection{Limitations}

One of the major limitations of this study is believed to be related to the inability to actually be involved in the details of the entire design process and have the ability to push back on subsystem designs (other than the propulsion system) and requirements, performing all of the trades that are mentioned. In order to achieve the kind of dynamic integrated system optimization desired, it is necessary to be intimately involved in the actual mission design process and have the freedom of adjusting various mission specifications. Essentially, the ideal process requires designing the entire mission. So, it is best suited to the role of a program manager to implement these trade and design evaluations.

Another issue, which affects the final propulsion system selections, is the quality and reliability of the cost estimation data and relationships. While they are reasonable and show appropriate trends, much of the data is nearly ten years old, or older and based on space

systems with very different characteristics from the kind one should expect of spacecraft 
to be flown ten or more years in the future. However, this is almost always a problem in predicting future trends. Additionally, all of the cost estimating relationships (for TFU, Launch and TRL) have variability and uncertainty that are very difficult to quantify. Therefore, placing much significance on specific numbers should be avoided. Emphasis in interpreting the results should focus more on propulsion system to propulsion system comparisons and trends while keeping in mind the assumptions made.

As far as modeling the propulsion systems is concerned, the assumptions made therein are also a source of limitation and some uncertainty. Data describing the spectral content of both FEEP and colloid thruster thrust variation are based on single experiments using laboratory prototypes in both cases. They are generally indicative, but large error bounds should be assumed when interpreting specific results. Special attention should, therefore, be paid to these assumptions, to prevent misleading conclusions from being drawn. The same kind of caution should be used in interpreting the detailed results of any of the propulsion system designs presented. Special attention should be paid to the design methodologies outlined in Section 5.1, and the detailed equations used to arrive at the design parameter values should be carefully viewed in Appendix C. If further understanding is sought and more detail required, the reader is directed to the sources of the relationships provided in the list of references.

\subsection{Future Work}

There are several areas in which this work can be expanded upon or refined. Of particular importance to interferometric missions appears to be the issues associated with detector contamination. At the current time relatively little information is available concerning the effects of particular thruster effluents on the highly sensitive optics with which many of the spacecraft are to be outfitted. Experimentation and theoretical work are needed to quantify the specific degradation effects that different propulsion systems will have on various materials in the space environment. This contamination issue may very well be one of the most stringent requirements that needs to be met by a propulsion system for 
these highly sensitive spacecraft missions. Information is necessary to characterize both spacecraft self contamination and spacecraft-spacecraft contamination resulting from close proximity formation flying.

Another potential area for more detailed work lies in a rather different topic. In order to further verify and validate the results gleaned from the propulsion system operational modeling simulations, it is felt that control algorithms similar to the type that would actually be used for the spacecraft control system should be integrated with the propulsion system characteristics (often described as models of actuator transient dynamics). This modeling, in conjunction with more data on the steady state behavior of the propulsion systems, could provide more authoritative and trustworthy results.

To address the issue raised in Section 7.2 concerning a more thorough optimization of the propulsion system with respect to an entire mission, a future study could be performed on a single mission. In doing so, not only is there the potential for adopting a more interactive methodology in the propulsion system design, but the greater detail in mission requirements and attributes it provides could allow a more rigorous and precise use of the GINA methodology for evaluating the overall system performance. 


\section{REFERENCES}

[Andrenucci, 1993] Andrenucci, M., Marcuccio, S. and Genovese, A. "The Use of FEEP Systems for Micronewton Thrust Level Missions," AIAA 93-2390, 29th AIAAV SAE/ASME/ASEE Joint Propulsion Conference and Exhibit, Monterrey, CA, June 1993.

[Beichman, 1997-1998] Beichman, C. A. (Content), Sorenson, Sugi and Kawasaki, Kristy (Design). "Terrestrial Planet Finder World Wide Web Site," http:// tpf.jpl.nasa.gov/, California Institute of Technology, 1997-1998.

[Beichman, 1999] Edited by Beichman, C. A., Woolf, N. J. and Lindensmith, C. A. “TPF: Terrestrial Planet Finder," JPL Publication 99-3, May 1999.

[Bender, 1998] Bender, P., Brillet, A., Ciufolini, I., Cruise, A. M., Cutler, C., Danzmann, K., Fidecaro, F., Folkner, W. M., Hough, J., McNamara, P., Peterseim, M., Robertson, D., Rodrigues, M., Rudiger, A., Sandford, M., Schafer, G, Schilling, R., Schutz, B., Speake, C., Stebbins, R. T., Sumner, T., Touboul, P., Vinet, J.-Y., Vitale, S., Ward, H., Winkler,W. "LISA: Laser Interferometry Space Antenna for the Detection and Observation of Gravitational Waves," Pre-Phase A Report, Second Edition, LISA Pre-Project Office, Jet Propulsion Laboratory, California Institute of Technology, July 1998.

[Blackwood, 1998] Blackwood, G.H., Dubovitsky, S., Linfield, R. P. and Gorham, P.W. "Interferometer Instrument Design for New Millennium Deep Space 3," Astronomical interferometry; Proceedings of the Meeting, Kona, HI, March, 1998. Pt. 1 (A98-40801 11-35), Society of Photo-Optical Instrumentation Engineers (SPIE Proceedings. Vol. 3350), p. 173-183, 1998.

[Blackwood, 1999] Blackwood, Gary. Presentation: "FY99 Third Quarter Review: Space Technology Three," Jet Propulsion Laboratory, California Institute of Technology, August 13, 1999.

[Blandino, 1998] Blandino, J. J. and Cassady, R. J. "Propulsion Requirements and Options for the New Millennium Interferometer (DS-3) Mission," AIAA 983331, 34th AIAA/ASME/SAE/ASEE Joint Propulsion Conference \& Exhibit, Cleveland, OH, July 1998.

[Burton, 1998] Burton, R. L. and Turchi, P. J. "Pulsed Plasma Thruster," Journal of Propulsion and Power, Vol. 14, No. 5 September-October 1998.

[Bzibziak, 2000] Bzibziak, Raymond. "Update of Cold Gas Propulsion at Moog," AIAA 2000-3718, 36th AIAA/ASME/SAE/ASEE Joint Propulsion conference and 
Exhibit, Huntsville, AL, July 2000.

[Cash (Web Site), 2000] Cash, Webster. "X-ray Interferometry: Science Fiction or Science Fact?," http://casa.colorado.edu/ wcash/interf/Interfere.htm, University of Colorado, Center for Astrophysics and Space Astronomy, accessed May, 2000.

[Cash, 2000] Cash, Webster. "MAXIM Preliminary Design," Work Supported by The NASA Institute for Advanced Concepts, August 2000.

[Fernandez de la Mora, 1994] Fernandez de La Mora, J., and Loscertales, I.G. "The Current Emitted by Highly Conducting Taylor Cones," Journal of Fluid Mechanics, Vol. 260, pp. 155-184, Cambridge University Press, 1994.

[Folkner, 1998] Folkner, W. M. and Sorensen, Sugi (Design). "Laser Interferometer Space Antenna World Wide Web Site," California Institute of Technology, 1998.

[Gamero, 2000] Gamero, Manuel. Communication with author. 13 June 2000.

[Grogan, 1998] Grogan, Robert L., Blackwood, Gary H. and Calvet, Robert J. "Optical Delay Line Nanometer Level Pathlength Control Law Design for Space-Based Interferometry," Proceedings of the Astronomical Interferometry Meeting (SPIE Proceedings. Vol. 3350), p. 14-25, Kona, HI, 1998.

[Gulczinski, 2000] Gulczinski, F. S., Dulligan, M. J., Lake, J. P. and Spanjers, G. G. "Micropropulsion Research at AFRL," AIAA 2000-3255, 36th AIAA/ASME/SAE/ ASEE Joint Propulsion Conference \& Exhibit, Huntsville, AL, July 2000.

[Guman, 1968] Guman, William J. "Pulsed Plasma Technology in Microthrusters," AFAPL-TR-68-132, Fairchild Hiller Corporation, Farmingdale, NY, November 1968.

[Guman, 1975] Guman, William J. "Designing Solid Propellant Pulsed Plasma Thrusters," AIAA 75-410, AIAA 11th Electric Propulsion Conference, New Orleans, LA, March 1975.

[Hruby, 2000] Hruby, Vlad. Personal communication (fax) to author: "Three $20 \mu \mathrm{N}$ Thrusters Cluster: Mass, Volume and Power Summaries." Busek Company, Inc., September 18, 2000.

[Jackson, 1972] Jackson, F.A., Zafran, S., Beynon, J. C., Kidd, P. W., Kreive, W. F., Shelton, H., Farber, B. F. and Hall, D. F. "Colloid Advanced Development Program, Interim Final Report No. 2," AFRPL-TR-72-73, TRW Systems Technical Report, August 1972.

[Jilla, 1998] Jilla, Cyrus D. "Separated Spacecraft Interferometry - System Architecture Design and Optimization," Massachusetts Institute of Technology M.S. Thesis in 
Aeronautics and Astronautics, December 1998.

[Kong,1998] Kong, M. C. "Optimal Trajectories and Orbit Design for Separated Spacecraft Interferometry," Massachusetts Institute of Technology M.S. thesis in Aeronautics and Astronautics, November 1998.

[Larson, 1992] Larson, Wiley J., Wertz, James R. eds. Space Mission Analysis and Design. Torrance, CA: Microcosm, Inc., 1992.

[Lau, 1999] Lau, Kenneth. Presentation Draft: "GN\&C Requirement Derivation from Interferometer Requirements Version 0.2 10/8/99," Jet Propulsion Laboratory, California Institute of Technology, October 8, 1999.

[Lawson,2000] Lawson, Peter. "Scientific American World Wide Web Site, Ask the Experts Page," Astronomy Archive, http://www.sciam.com/askexpert/astronomy/ astronomyl.html, accessed May, 2000.

[Leisawitz (Web site), 2000] Leisawitz, David. "SPECS World Wide Web Site," http:// space.gsfc.nasa.gov/astro/specs/, Goddard Spaceflight Center, Astrophysics Data Facility, revised April 6, 2000.

[Leisawitz, 2000] Leisawitz, D., Danchi, W., DiPirro, M., Feinberg, L., Gezari, D., Hagopian, M., Langer, W., Mather, J., Moseley, S., Shao, M., Silverberg, R., Staguhn, J., Swain, M., Yorke, H., and Zhang, X. "Scientific Motivation and Technology Requirements for the SPIRIT and SPECS Far-infrared/Submillimeter Space Interferometers," UV, Optical, and IR Space Telescopes and Instruments, Proc. SPIE 4013, pp. 327-354, 2000.

[London, 1996] London, Adam P. "A Systems Study of Propulsion Technologies for Orbit and Attitude Control of Microspacecraft." Massachusetts Institute of Technology M.S. Thesis in Aeronautics and Astronautics, May 1996.

[Mankins, 1995] Mankins, J. C. “Technology Readiness Levels: A White Paper," http:// rel.jpl.nasa.gov/Org/5053/qual/tecready.htm, Advanced Concepts Office, Office of Space Access and Technology, NASA, April 6, 1995.

[Marcuccio, 1998] Marcuccio, S., Genovese, A. and Andrenucci, M. "Experimental Performance of Field Emission Microthrusters," Journal of Propulsion and Power, Vol. 14, No. 5, September- October 1998.

[Marcuccio, 2000] Marcuccio, S., Nicolini, D., Saviozzi, M. "Endurance Test of the Micronewton FEEP Thruster," AIAA 2000-3270, 36th AIAA/ASME/SAE/ASEE Joint Propulsion Conference \& Exhibit, Huntsville, AL, July 2000.

[Martinez-Sanchez, 1999a] Martinez-Sanchez, Manuel. "Colloidal Thruster Ion Extrac- 
tion Physics," Graduate Course in Space Propulsion (16.522), Massachusetts Institute of Technology, Cambridge, Massachusetts, May 13, 1999.

[Martinez-Sanchez, 1999b] Martinez-Sanchez, M., Fernandez de la Mora, J., Hruby, V., Gamero-Castano, M. and Khayms, V. "Research on Colloid Thrusters," IEPC 99-014, 26th International Electric Propulsion Conference, Kitakyushu, Japan, October 1999.

[Mather, 1998] Mather, J. C., Moseley, S. H. Jr., Leisawitz, D., Dwek, E., Hacking, P., Harwit, M., Mundy, L. G., Mushotzky, R. F., Neufeld, D., Spergel, D., Wright, E. L. "The Submillimeter Frontier: A Space Science Imperative," Rev. Sci. Instr, submitted (astro-ph/9812454).

[MAXIM Web Site, 2001] Eng, Eunice (questions), Tyler, Pat (design). "MAXIM World Wide Web Site," http://maxim.gsfc.nasa.gov/, Goddard Space Flight Center, NASA, accessed January 2001.

[Mikellides, 1999] Mikellides, Yiangos G. "Theoretical Modeling and Optimization of Ablation-Fed Pulsed Plasma Thrusters," The Ohio State University Ph.D. thesis in Aeronautical and Astronautical Engineering, 1999.

[Mueller, 2000] Mueller, Juergen. "Overview of Micropropulsion Technologies," Air Force Office of Scientific Research (AFOSR) Space Propulsion Contractors Meeting Presentation, San Francisco, CA, August 2000.

[Phinney, 2000] Phinney, E.S. et al. LISA Presentation View Graphs, June 2000.

[Shao, 2000] M. Shao, W. Danchi, M. DiPirro, M. Dragovan, L. Feinberg, M. Hagopian, W. Langer, C. Lawrence, P. Lawson, D. Leisawitz, J. Mather, S. H. Moseley, M. Swain, H. Yorke, X. Zhang, "Space-based Interferometric Telescopes for the FarInfrared," Interferometry in Optical Astronomy, Proc. SPIE 4006, 2000.

[Shaw, 1998] Shaw, G. B. "The Generalized Information Network Analysis Methodology for Distributed Satellite Systems," Massachusetts Institute of Technology Sc.D. Thesis in Aeronautics and Astronautics, October 1998.

[Spencer, 2000] Spencer, David T. "New Millennium Program Space Technology 6 Technology Announcement," http://nmp.jpl.nasa.gov/st6/st6_ta.pdf, California Institute of Technology, October, 10, 2000.

[ST-3 Web Site, 2001] ST3 Education and Public Outreach, "Space Technology 3 World Wide Web Site," http://nmp.jpl.nasa.gov/st3/index.html, California Institute of Technology, accessed January 2001.

[Steiger, 2000] Steiger, W., Genovese, A. and Tajmar, M. "Micronewton Indium FEEP Thrusters," Proceedings of the 3rd International Conference of Spacecraft Pro- 
pulsion, Cannes, France, October 2000.

[Tajmar, 2000] Tajmar, M., Steiger, W. and Genovese, A. "Austrian Research Centers Seibersdorf Electric Propulsion Activities," Conference on Electric Propulsion and Space Diagnostics, Massachusetts Institute of Technology, November, 2000.

[Taylor, 1964] Taylor, G. I. "Disintegration of Water Drops in an Electric Field," Proc. R. Soc., London A 280, 383-397, 1964.

[Turchi, 1999] Turchi, P.J., Mikellides, I.G, Mikellides, P.G. and Kamhawi, H. "Optimization of Pulsed Plasma Thrusters for Microsatellite Propulsion," AIAA 99-2301, 35th AIAA/ASME/SAE/ASEE Joint Propulsion Conference and Exhibit, Los Angeles, CA, June 1999.

[Vincent, 1999] Vincent, Mark A., Folkner, William M., Buchman, Saps, Stebbins, Robin T., Hruby, Vlad, Martinez-Sanchez, Manuel, Fernandez De La Mora, Juan. "Disturbance Reduction System Proposed for New Millennium Space Technology 5, A Phase-A Study Report," Jet Propulsion Laboratory, California Institute of Technology, July 1999.

[Vondra,1974] Vondra, R.J. and Thomassen, K. I. "Flight Qualified Pulsed Electric Thruster for Satellite Control," Journal of Spacecraft and Rockets, Vol. 11, No. 9, pp.613-617, September 1974.

[Zeleny, 1917] Zeleny, J. "Instability of Electrified Liquid Surfaces," Phys. Rev. 10, pp. 1-6, 1917. 
REFERENCES 


\section{Appendix A}

\section{SIMULATION SCRIPTS}

\section{A.1 PPT Simulation Script Example}

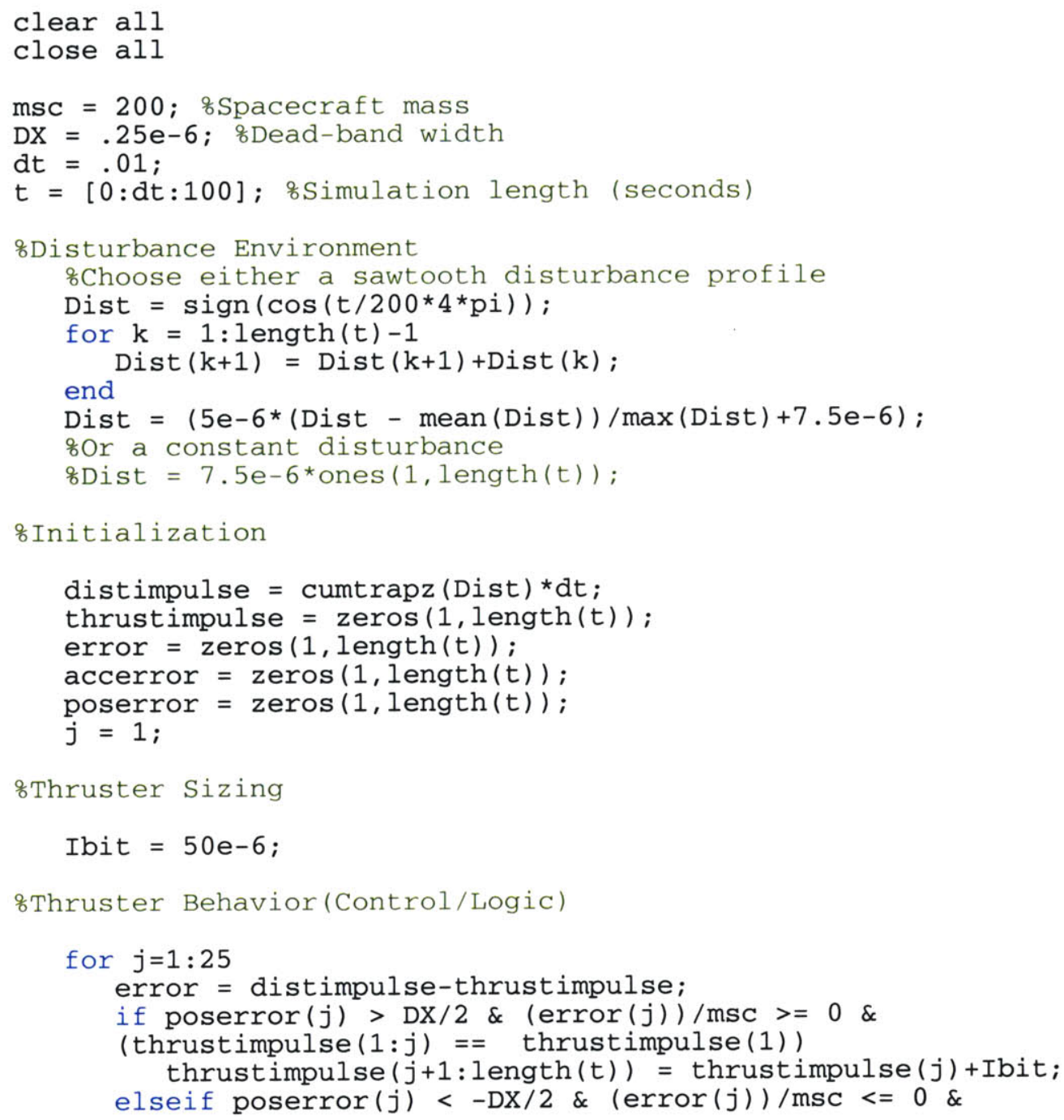




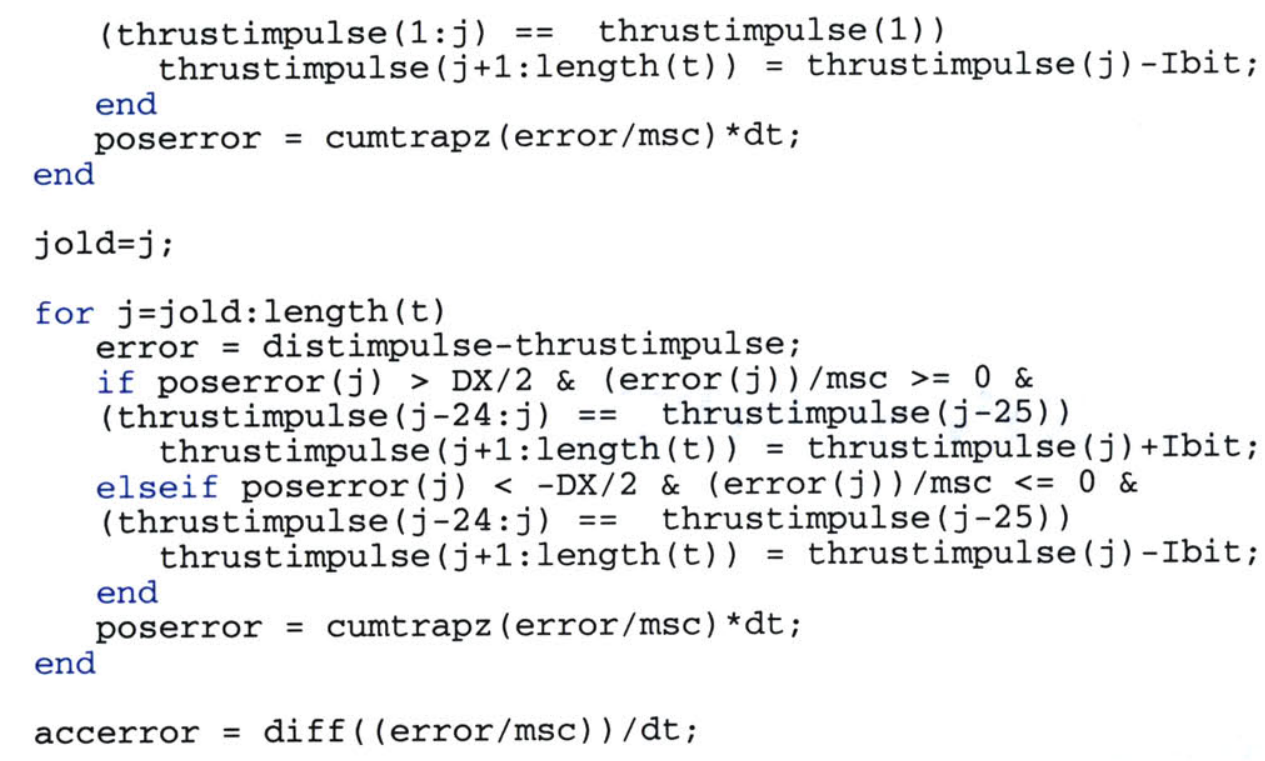

\&spectral Disturbance Analysis

$[P, F]=$ spectrum (poserror, $($ length $($ poserror $)),[],[], 1 / d t)$; $\mathrm{dF}=\operatorname{mean}(\operatorname{diff}(\mathrm{F})) ;$ $\operatorname{psd}(1,:)=P(:, 1)^{\prime} / /$ length $(P(:, 1)) / d F$;

$[\mathrm{Pa}, \mathrm{Fa}]=$ spectrum (accerror, $($ length (accerror) $),[],[], 1 / \mathrm{dt})$; $\mathrm{dFa}=\operatorname{mean}(\operatorname{diff}(\mathrm{Fa}))$; $\operatorname{psda}(1,:)=\operatorname{Pa}(:, 1)^{\prime} / / \operatorname{length}(\mathrm{Pa}(:, 1)) / \mathrm{dFa}$;

\%plots plot ( $t$, thrustimpulse); hold; plot ( $t$, distimpulse, ' $r$ ') ; title('Impulse vs. Time (50 \mu Ns PPT)'); xlabel ('Time [s]'); ylabel ('Impulse [Ns]') legend('Thruster', 'Disturbance', 0) ;

figure;

plot ( $t$,poserror, ' $\left.k^{\prime}\right)$; title('Trajectory (50 \mu Ns PPT)'); xlabel ('Time [s]'); ylabel ('Position [m]'); hold; $\operatorname{plot}\left(t, D X / 2\right.$ *ones $(1$, length $\left.(t)), ' r^{\prime}\right)$; $\operatorname{plot}\left(t,-\mathrm{Dx} / 2{ }^{*}\right.$ ones $(1$, length $\left.(t)), r^{\prime}\right)$;

figure; $\log \log (\mathrm{F}, \mathrm{psd})$; title('Position Power Spectral Density (50 \mu Ns PPT)'); xlabel ('Frequency $[\mathrm{Hz}]$ ') ;

ylabel('Position Power Density $\left[\mathrm{m}^{\wedge} 2 / \mathrm{Hz}\right] '$ );

figure; $\log \log (\mathrm{Fa}, \mathrm{psda})$; title('Acceleration Power Spectral Density (50 \mu Ns PPT)'); xlabel ('Frequency [Hz]'); ylabel ('Acceleration Power Density $\left[\left(\mathrm{m} / \mathrm{s}^{\wedge} 2\right)^{\wedge} 2 / \mathrm{Hz}\right]$ ') ;

\section{figure;}


plot (t (1: length (t) -1), diff (thrustimpulse)/dt) ;

title('Thrust Signal (50 \mu Ns PPT)');

xlabel ('Time [s]');

ylabel ('Thrust [N]');

figure;

plot ( $t$, Dist) ;

title('Disturbance Force (50 \mu Ns PPT)');

xlabel ('Time [s]');

ylabel ('Force $[\mathrm{N}]$ ') ;

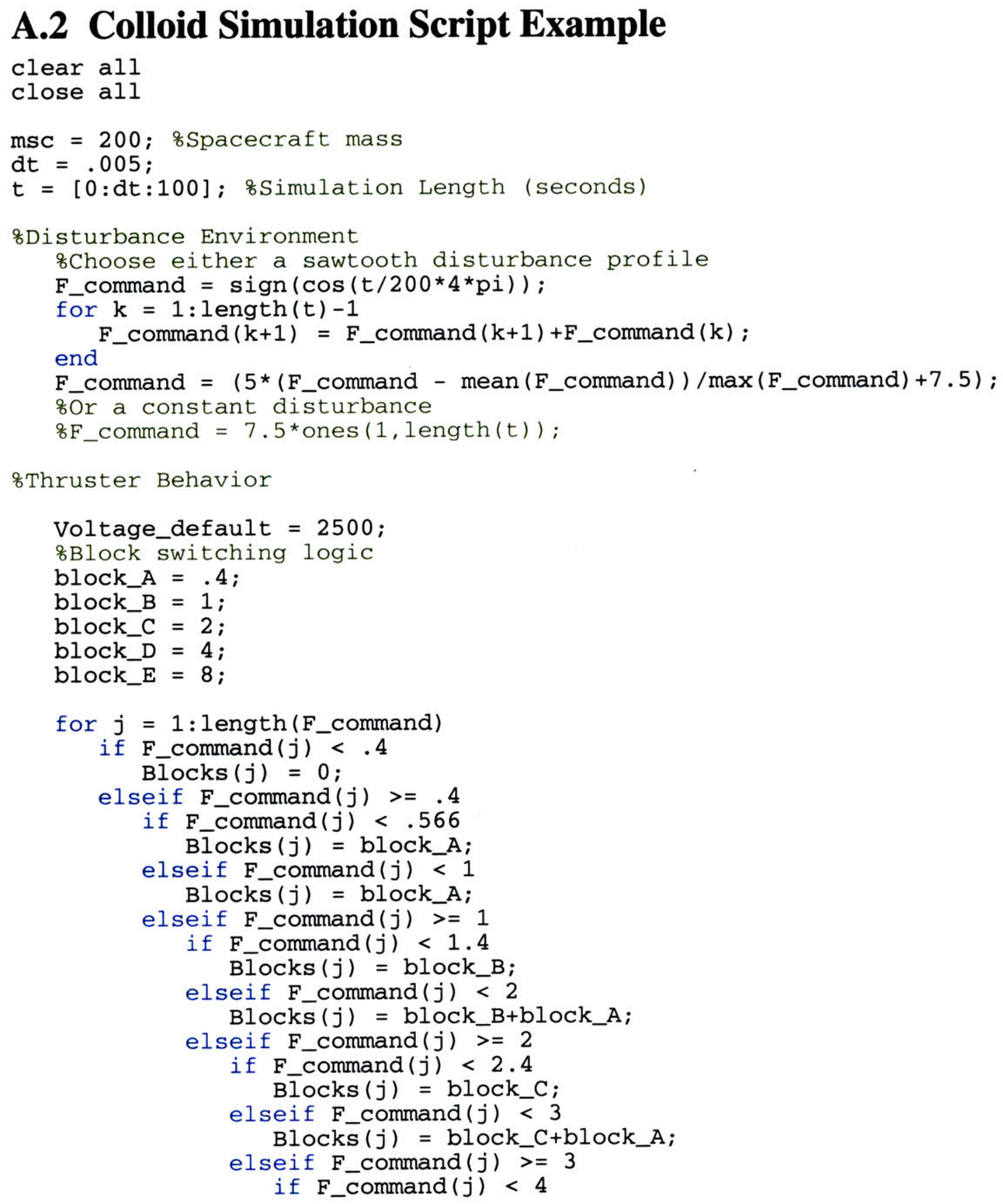




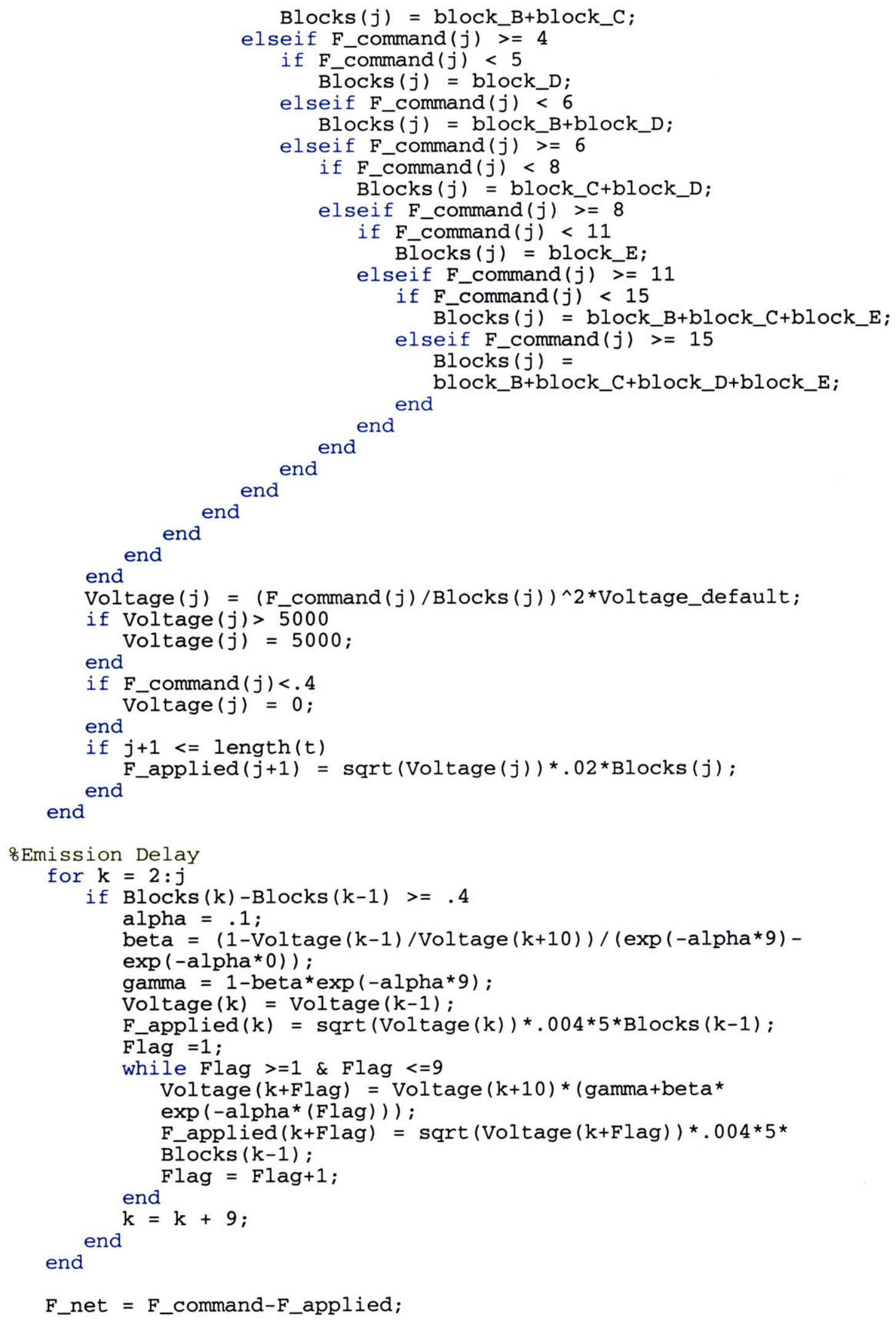




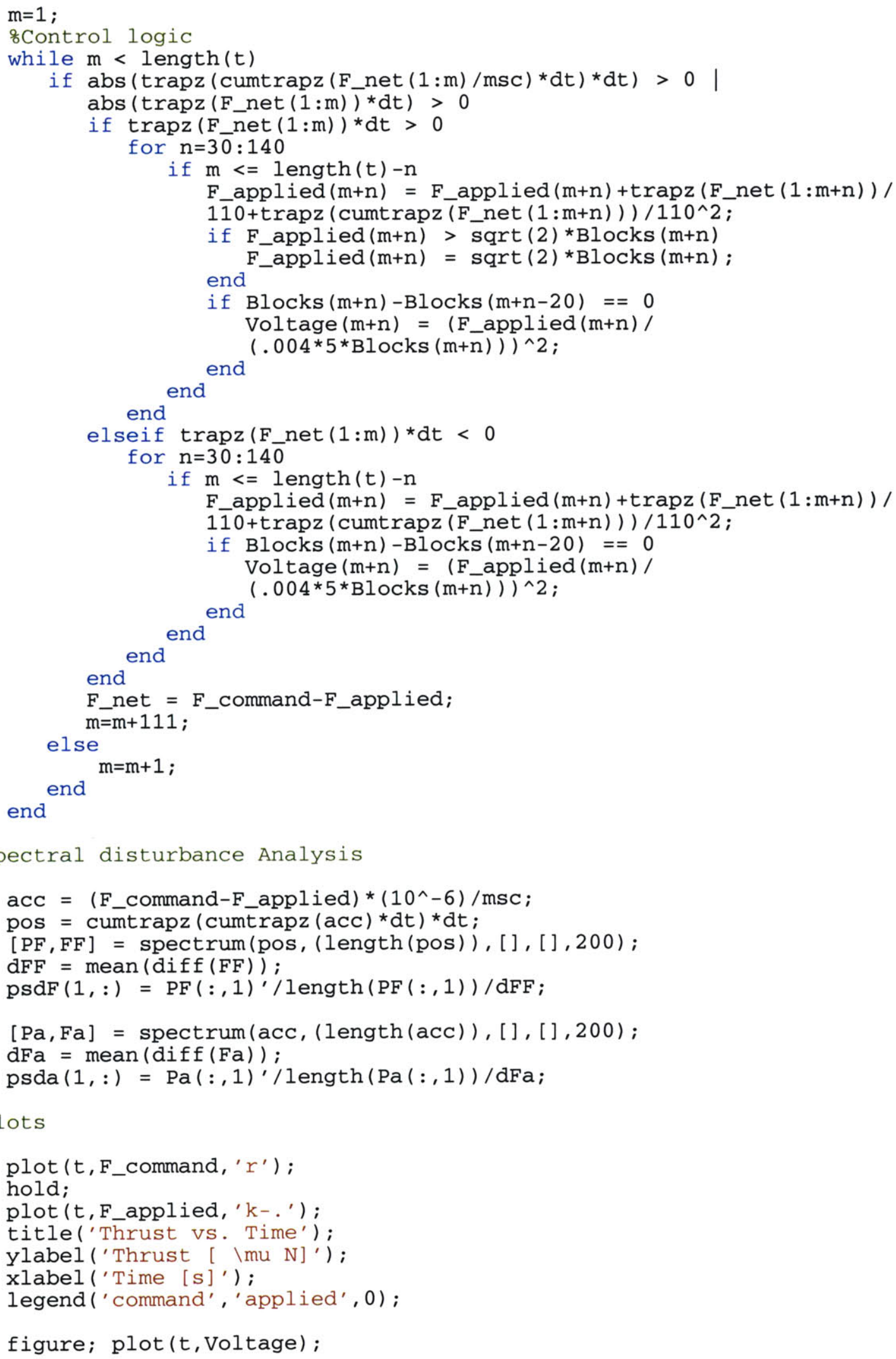




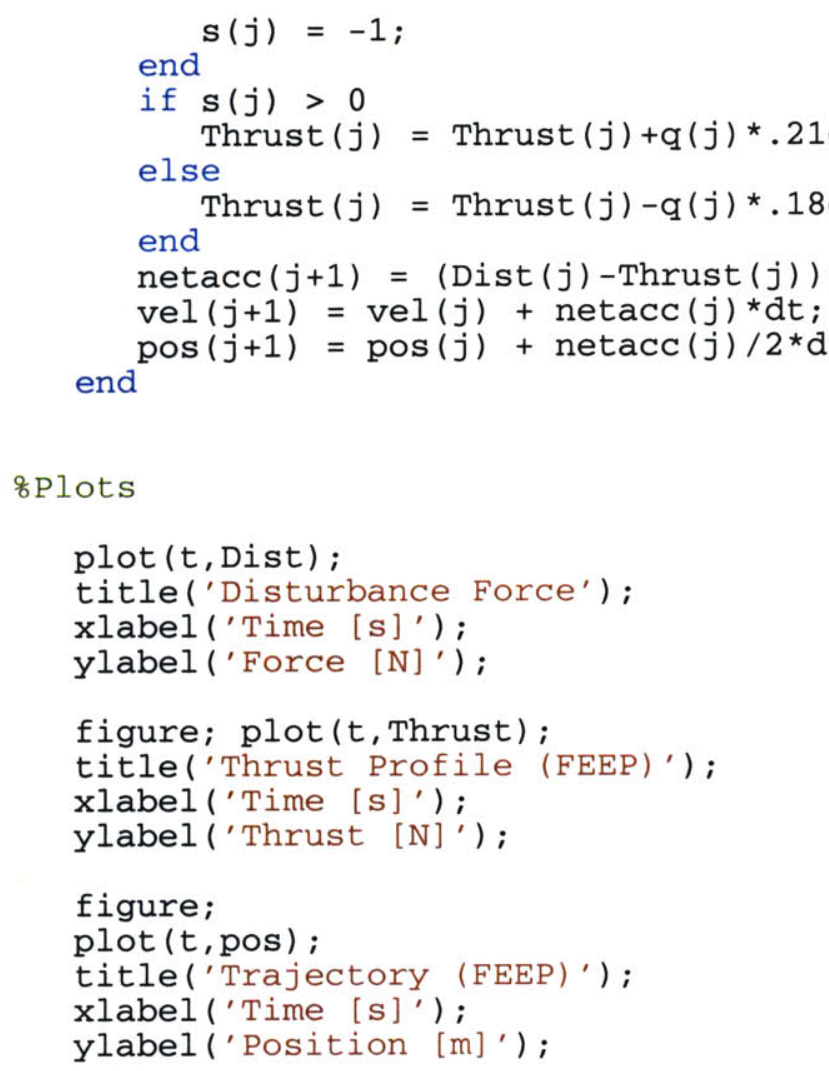

\section{A.4 Cold Gas Simulation Script Example}

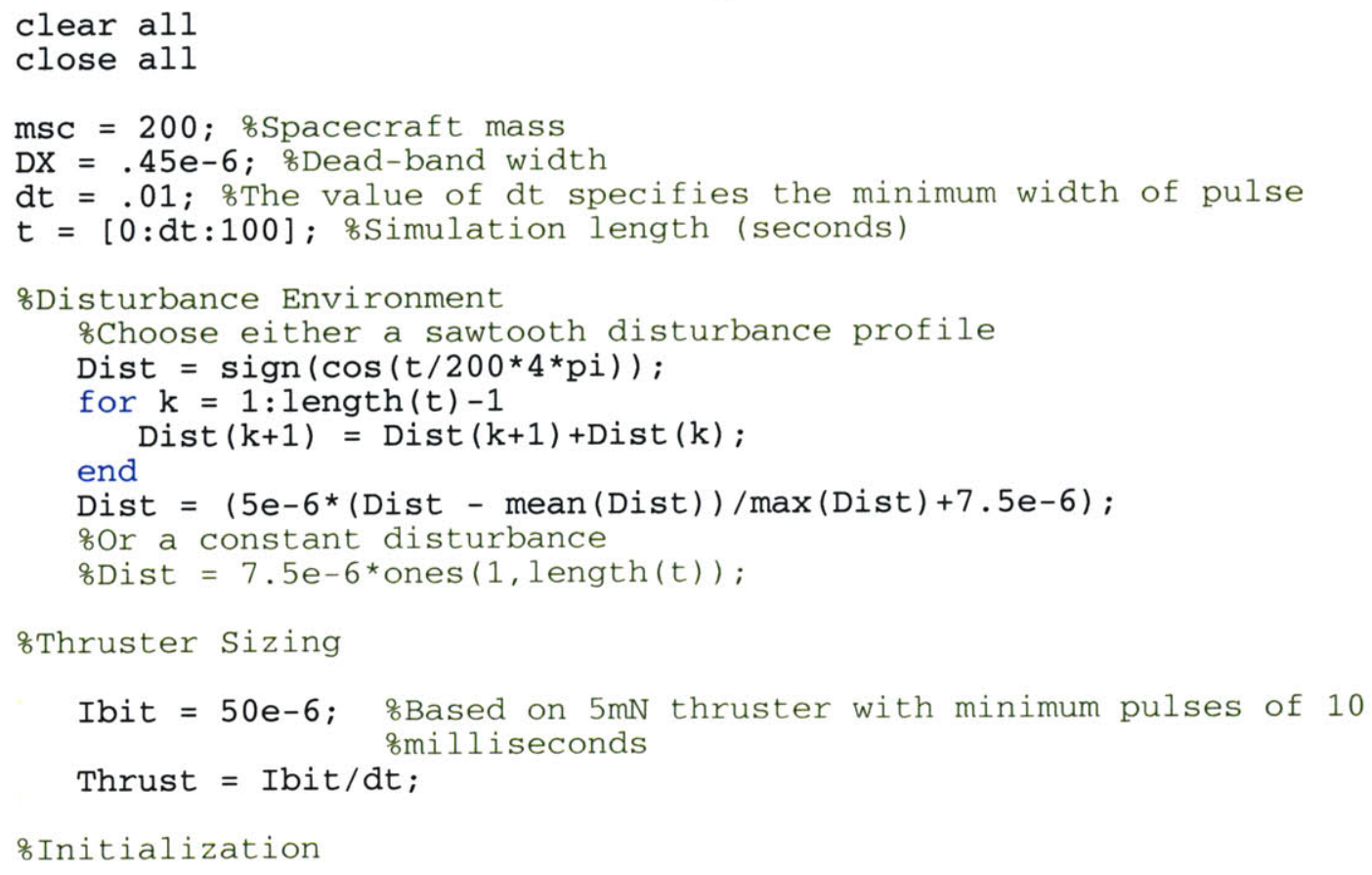




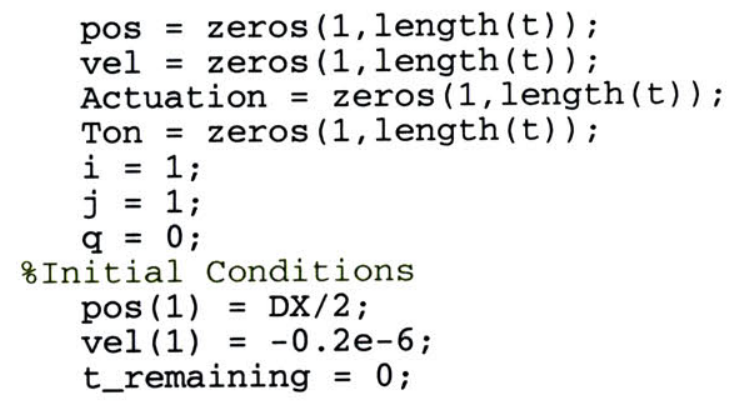

8Thruster behavior (Control/Logic)

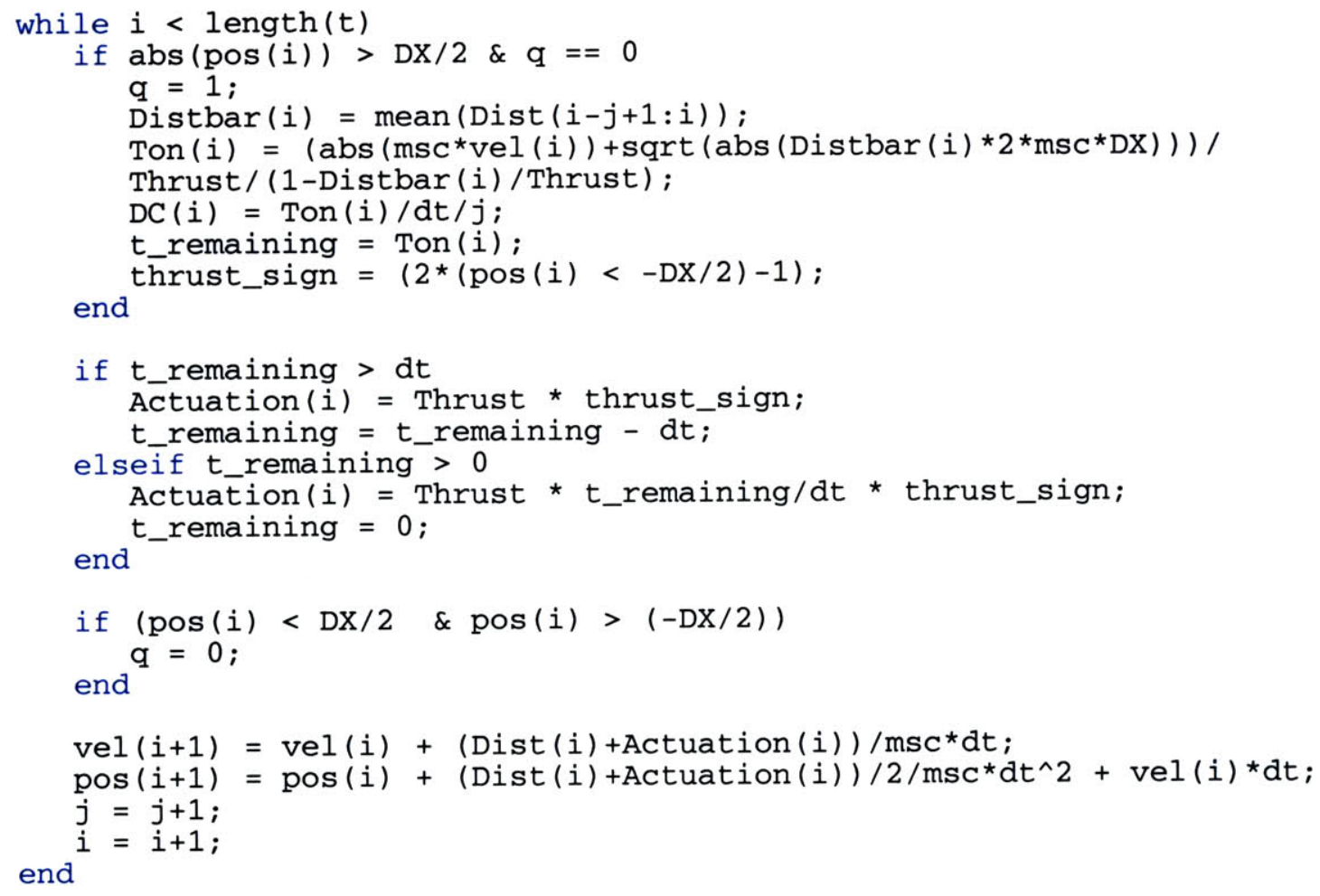

8Spectral disturbance Analysis

$[P, F]=\operatorname{spectrum}($ pos, $($ length $($ pos $)),[],[], 1 / d t) ;$

$\mathrm{dF}=\operatorname{mean}(\operatorname{diff}(\mathrm{F})) ;$

$\operatorname{psd}(1,:)=P(:, 1) ' /$ length $(P(:, 1)) / d F$;

acc $=($ Dist + Actuation $(1:$ length $(t))) /$ msc;

$[\mathrm{Pa}, \mathrm{Fa}]=\operatorname{spectrum}(\mathrm{acc},($ length $(\mathrm{acc})),[],[], 1 / \mathrm{dt})$;

$\mathrm{dFa}=\operatorname{mean}(\operatorname{diff}(\mathrm{Fa})) ;$

$\operatorname{psda}(1,:)=\mathrm{Pa}(:, 1)$ '/length $(\mathrm{Pa}(:, 1)) / \mathrm{dFa}$;

\&Plots

plot (t,Dist);

title('Disturbance Force');

xlabel ('Time [s]');

ylabel ('Force $[\mathrm{N}]$ ') ; 
figure; plot(t,Actuation(1:length(t)));

title('Thrust Profile $(5 \mathrm{mN}, 50 \backslash \mathrm{mu}$ Ns Impulse Bit Cold Gas

Thruster)');

xlabel ('Time [s]') ;

ylabel ('Thrust $[\mathrm{N}]$ ');

figure;

plot $(t, \operatorname{pos}(1:$ length $(t)))$;

title('Trajectory ( $5 \mathrm{mN}, 50 \backslash \mathrm{mu}$ Ns Impulse Bit Cold Gas Thruster)');

xlabel ('Time [s]');

ylabel ('Position [m]') ;

hold; $\operatorname{plot}\left(t, \mathrm{DX} / 2\right.$ *ones $(1$, length $\left.(t)), \mathrm{r}^{\prime}\right)$;

$\operatorname{plot}\left(t,-\mathrm{DX} / 2{ }^{*}\right.$ ones $(1$, length $\left.(t)),{ }^{\prime}{ }^{\prime}\right)$;

figure;

$\log \log (\mathrm{F}, \mathrm{psd})$;

title('Position Power Spectral Density (Cold Gas)');

xlabel ('Frequency $[\mathrm{Hz}]$ ') ;

ylabel('Position Power Density [m^2/Hz]');

figure;

$\log \log (\mathrm{Fa}, \mathrm{psda})$;

title('Acceleration Power Spectral Density (Cold Gas)');

xlabel ('Frequency [Hz]');

ylabel('Acceleration Power Density $\left[\mathrm{m} / \mathrm{s}^{\wedge} 2 \mathrm{~Hz}\right]$ '); 
APPENDIX A 


\section{Appendix B}

\section{SIMULATION RESULTS}

\section{B.1 ST-3 Simulation Results}

\section{B.1.1 PPT}
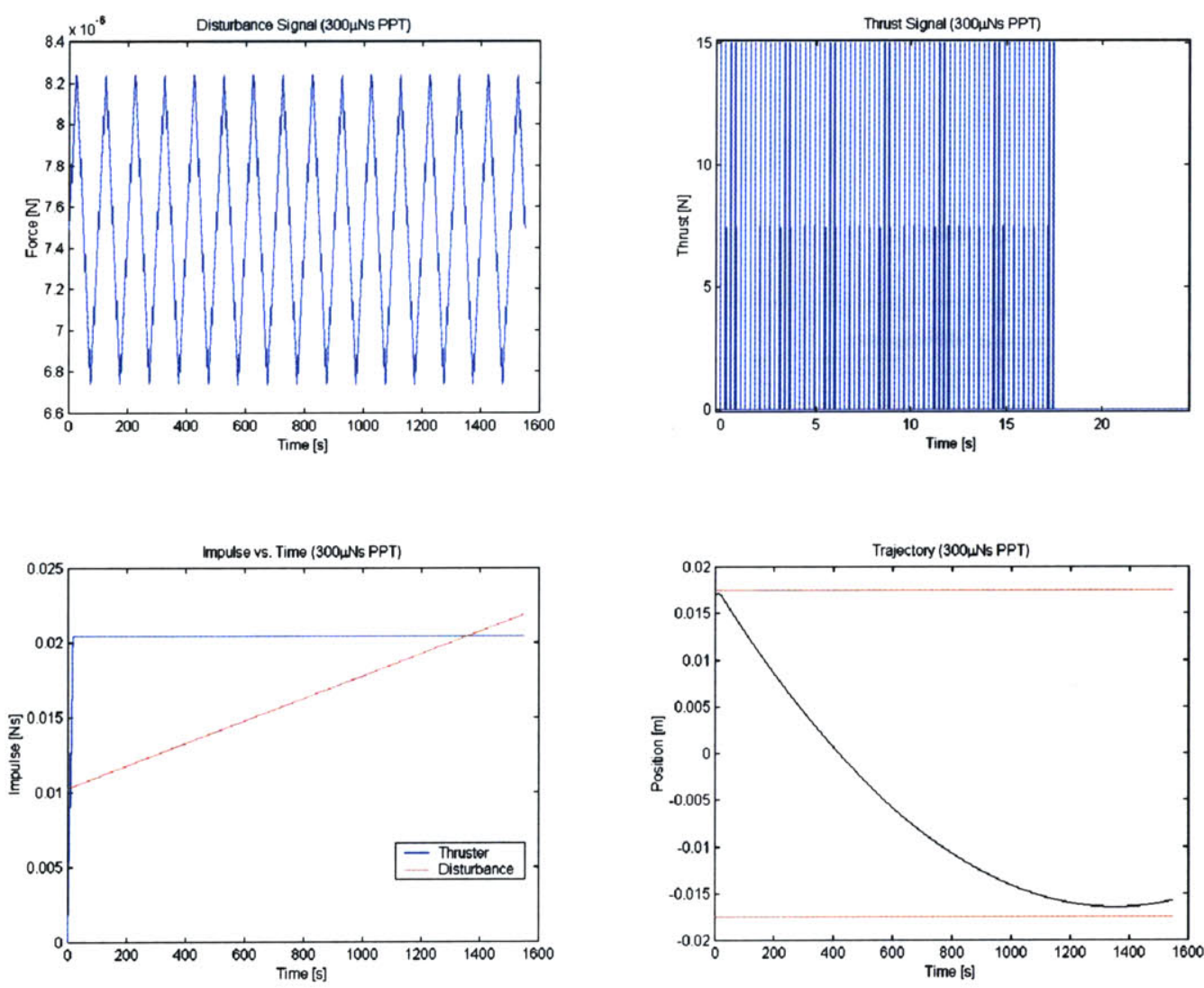

Figure B.1 Simulation results for the ST-3 collector spacecraft using PPTs. 

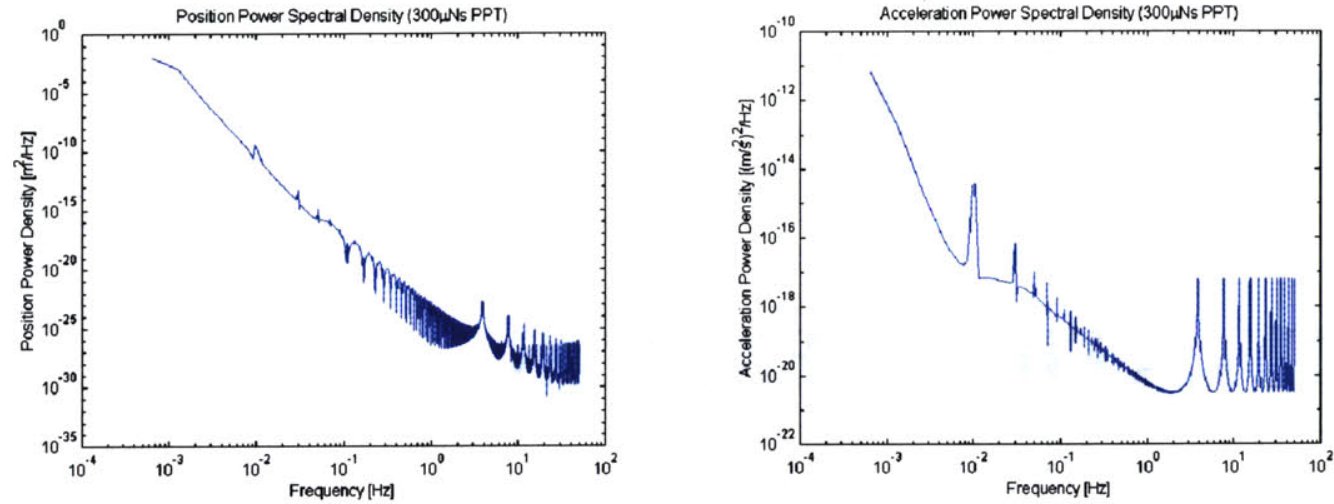

Figure B.1 Simulation results for the ST-3 collector spacecraft using PPTs.
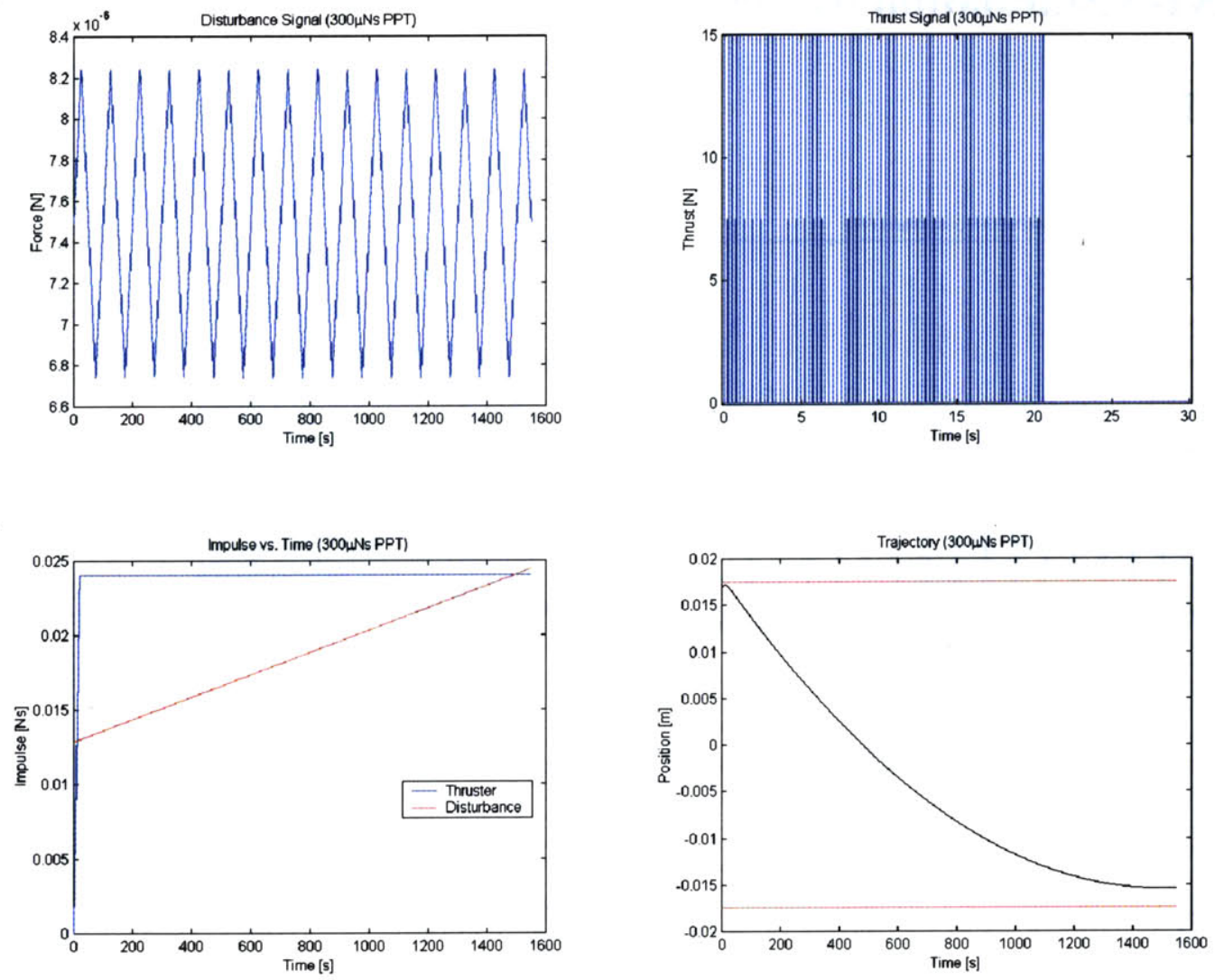

Figure B.2 Simulation results for the ST-3 combiner spacecraft using PPTs. 

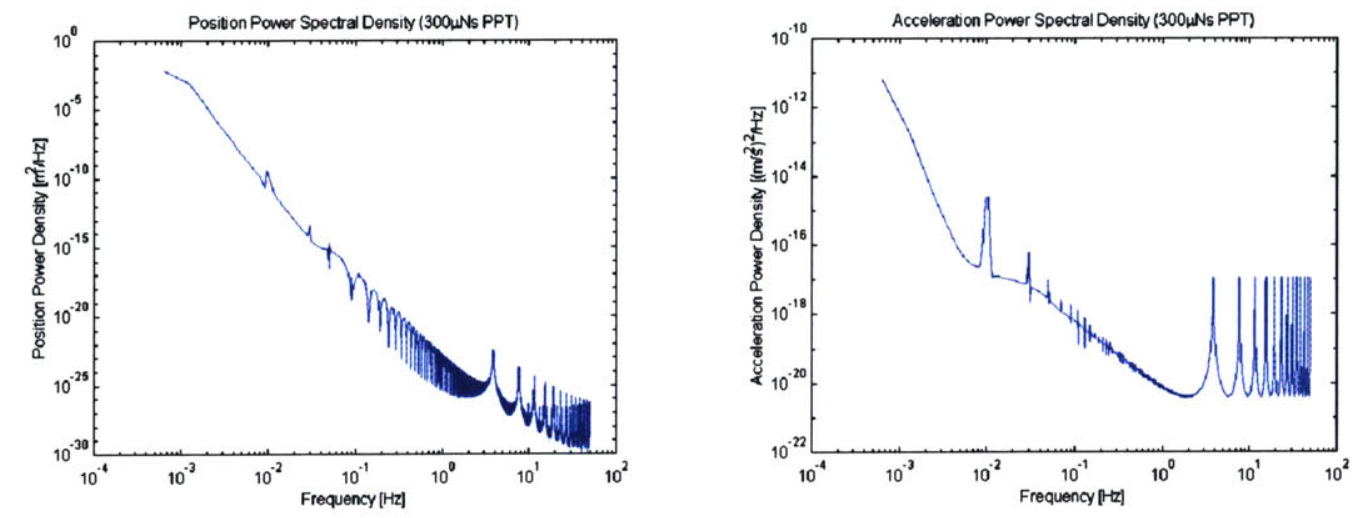

Figure B.2 Simulation results for the ST-3 combiner spacecraft using PPTs.

\section{B.1.2 Colloid}
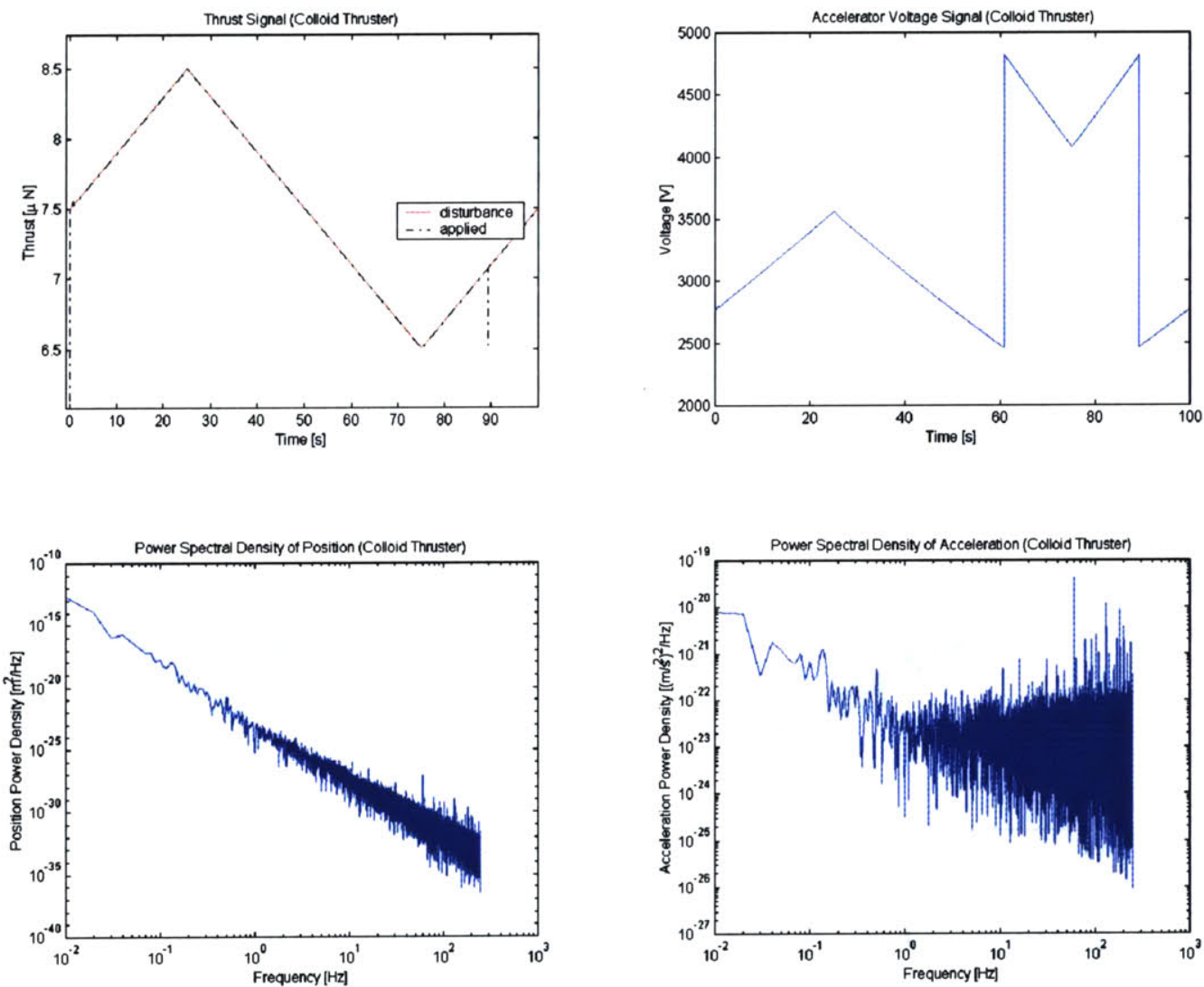

Figure B.3 Simulation results for the ST-3 collector spacecraft using colloid thrusters. 


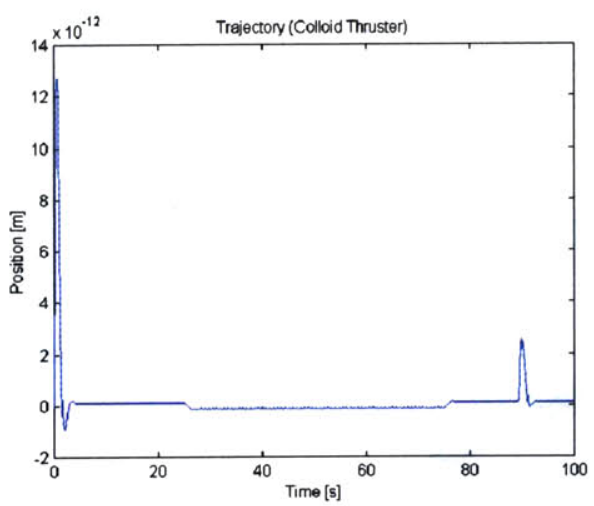

Figure B.3 Simulation results for the ST-3 collector spacecraft using colloid thrusters.
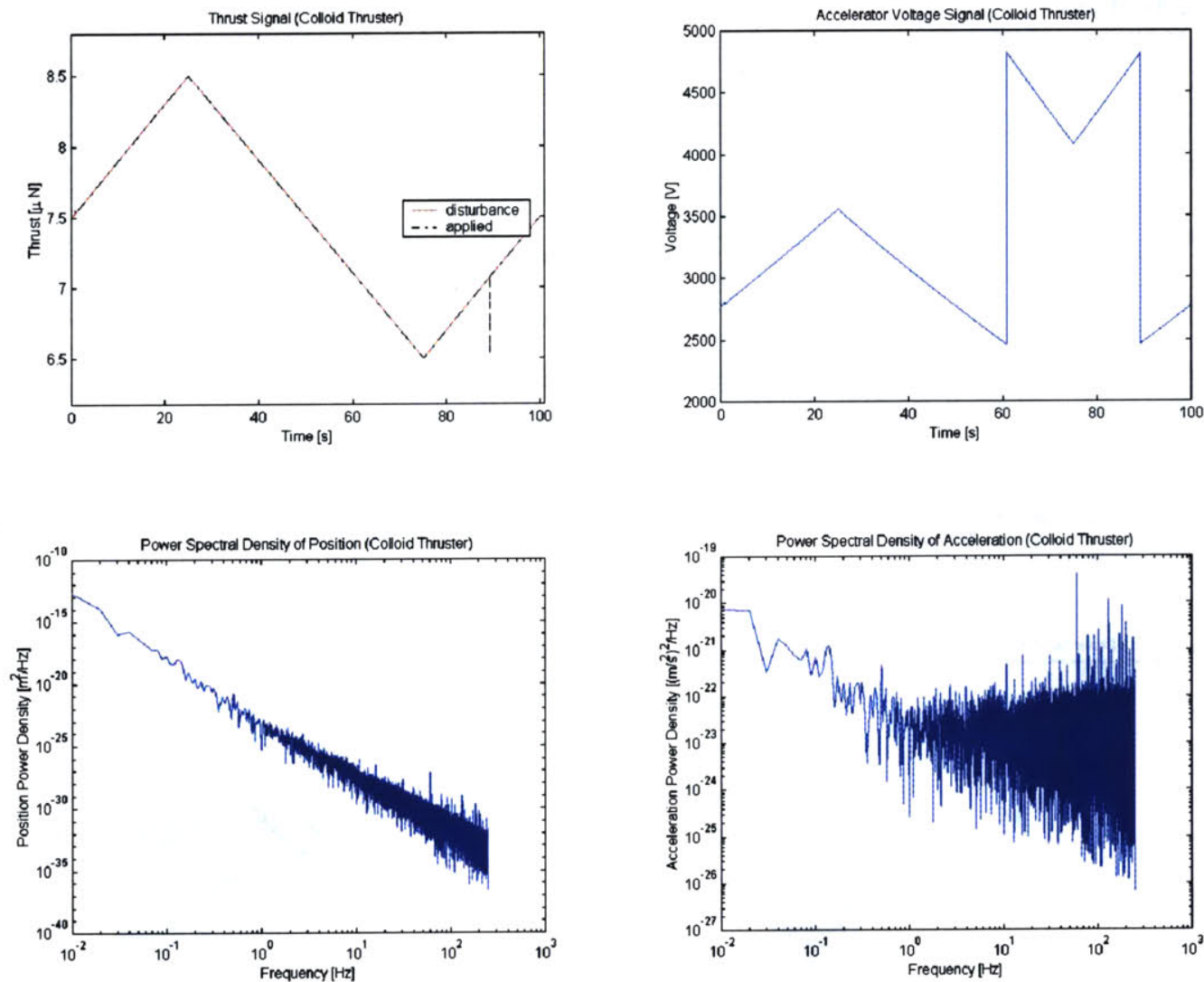

Figure B.4 Simulation results for the ST-3 combiner spacecraft using colloid thrusters. 


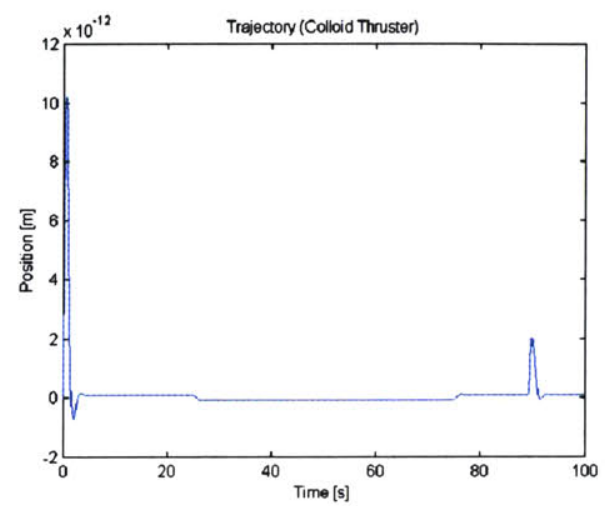

Figure B.4 Simulation results for the ST-3 combiner spacecraft using colloid thrusters.

\section{B.1.3 FEEP}
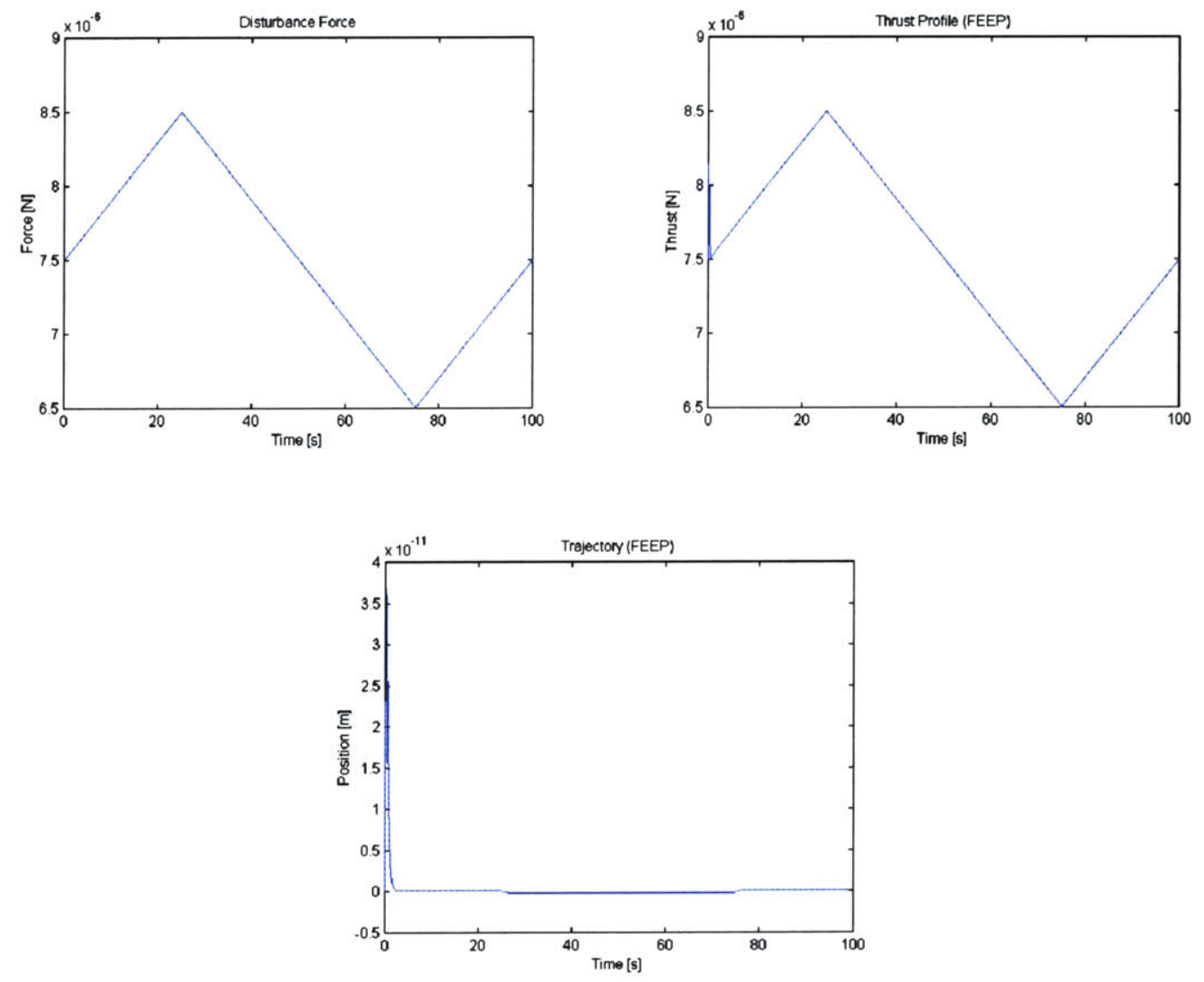

Figure B.5 Simulation results for the ST-3 collector spacecraft using a FEEP system. 

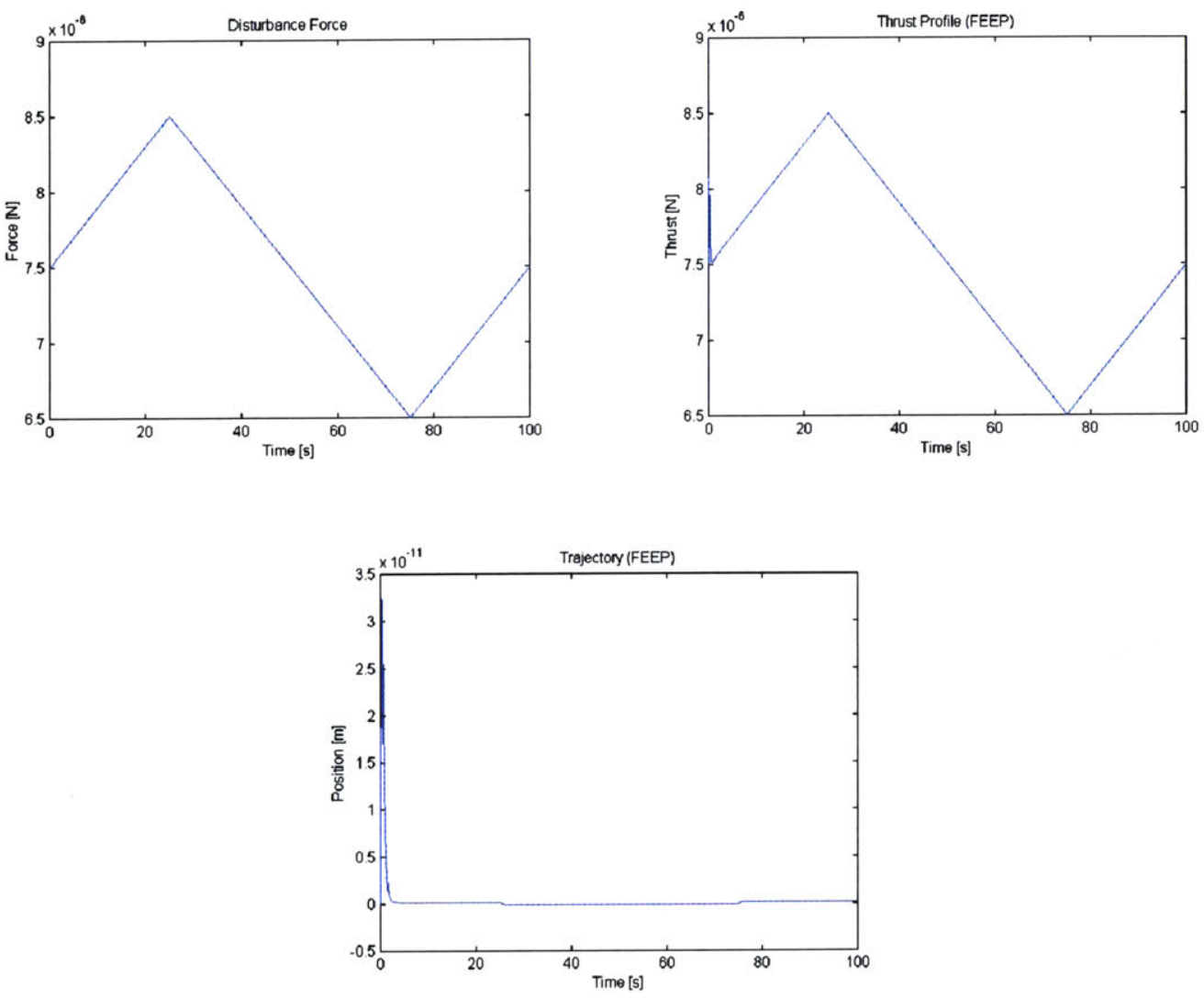

Figure B.6 Simulation results for the ST-3 combiner spacecraft using a FEEP system.

\section{B.1.4 Cold Gas}
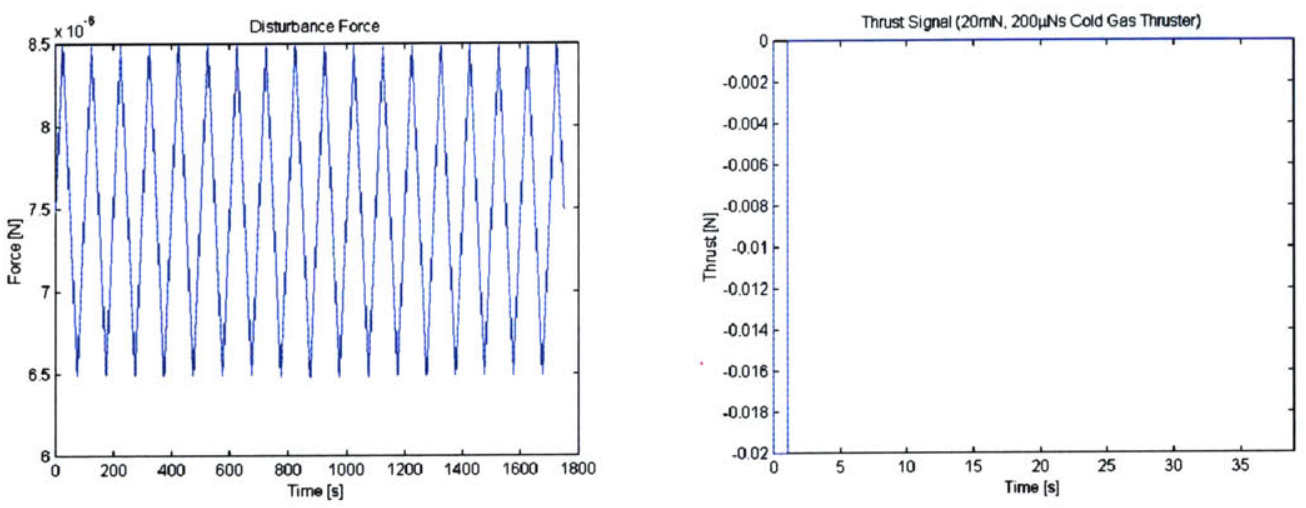

Figure B.7 Simulation results for the ST-3 collector spacecraft using cold gas thrusters. 

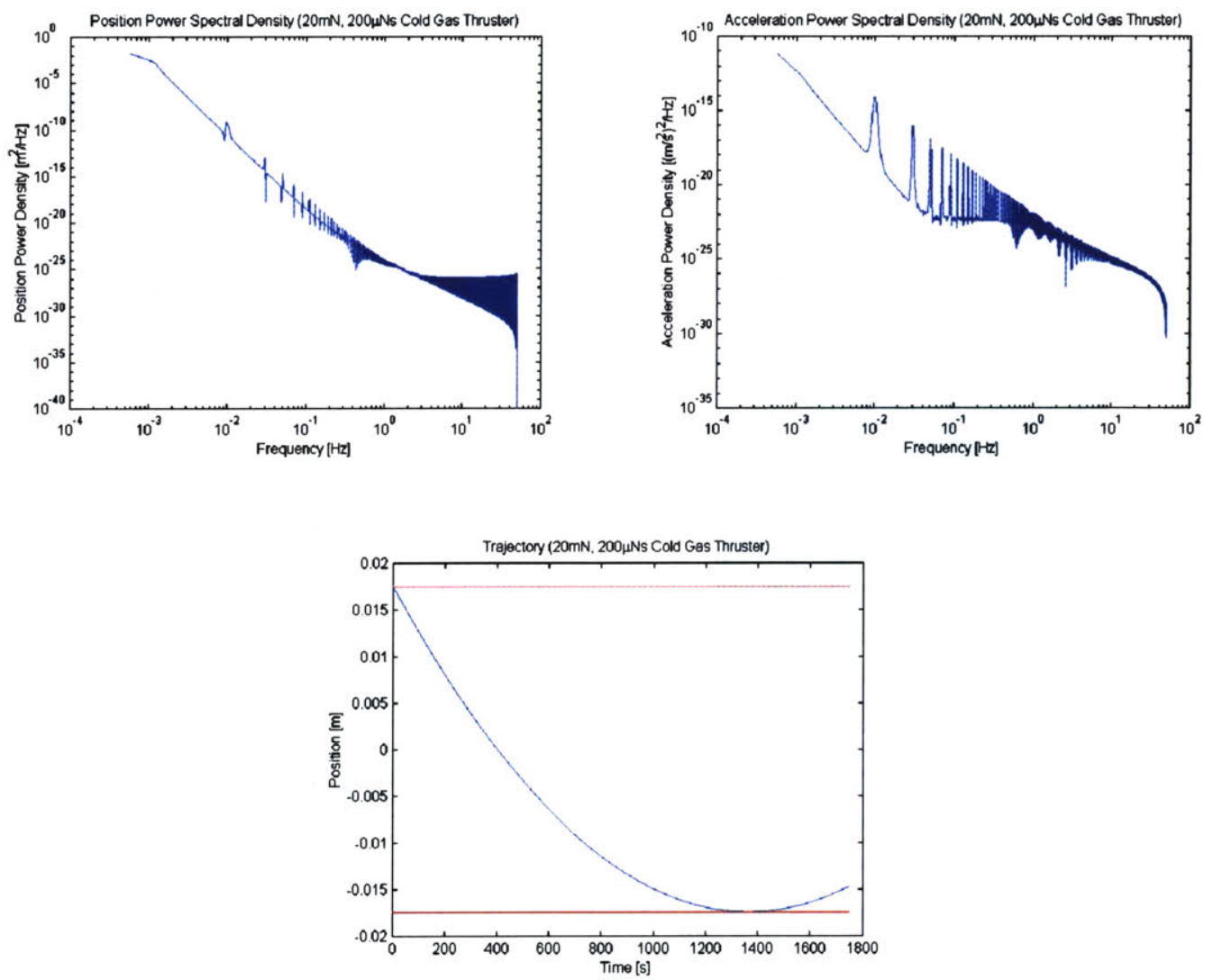

Figure B.7 Simulation results for the ST-3 collector spacecraft using cold gas thrusters.
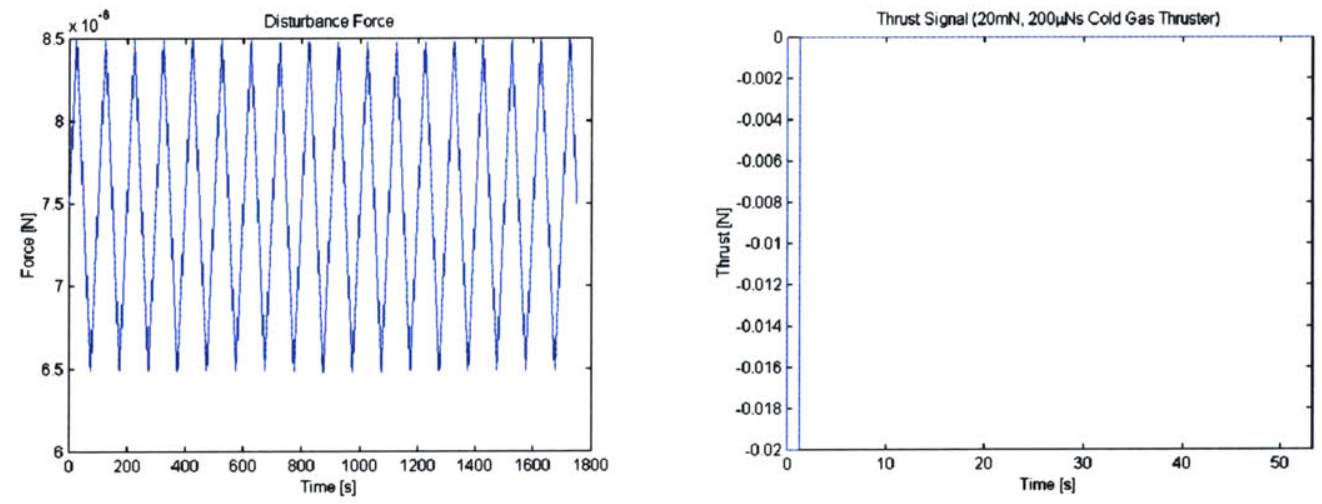

Figure B.8 Simulation results for the ST-3 combiner spacecraft using cold gas thrusters. 

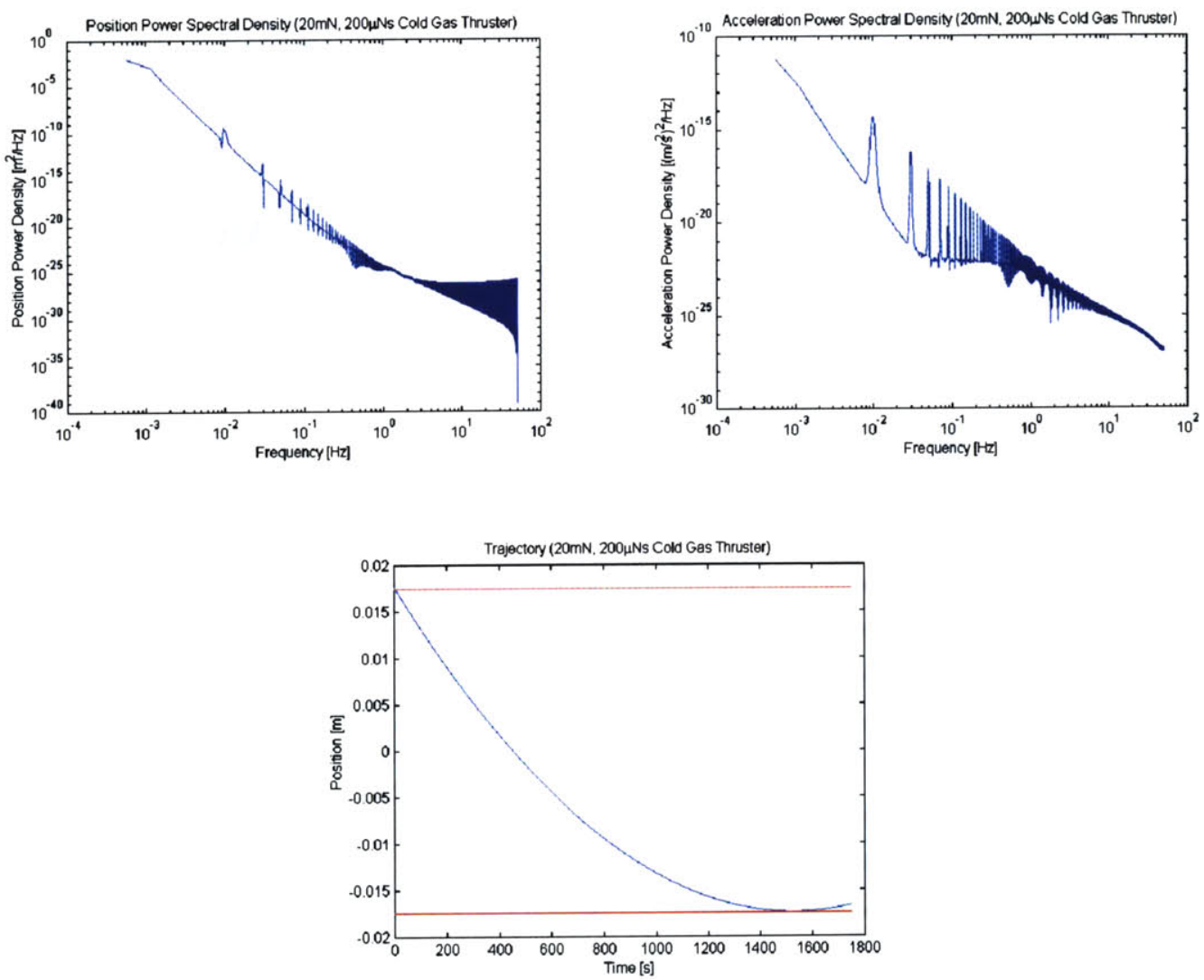

Figure B.8 Simulation results for the ST-3 combiner spacecraft using cold gas thrusters. 


\section{B.2 TPF Simulation Results}

\section{B.2.1 PPT}
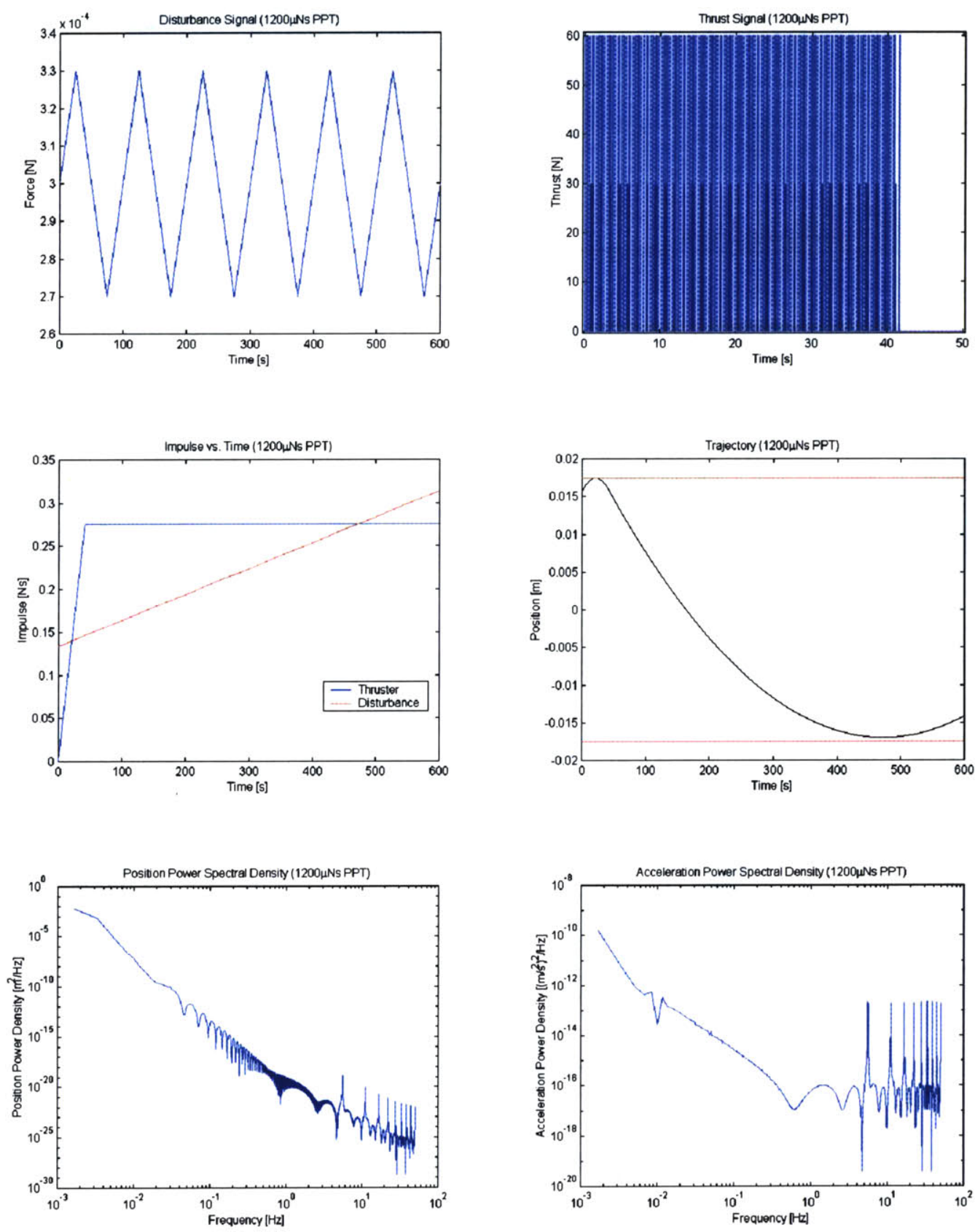

Figure B.9 Simulation results for the TPF collector spacecraft using PPTs. 

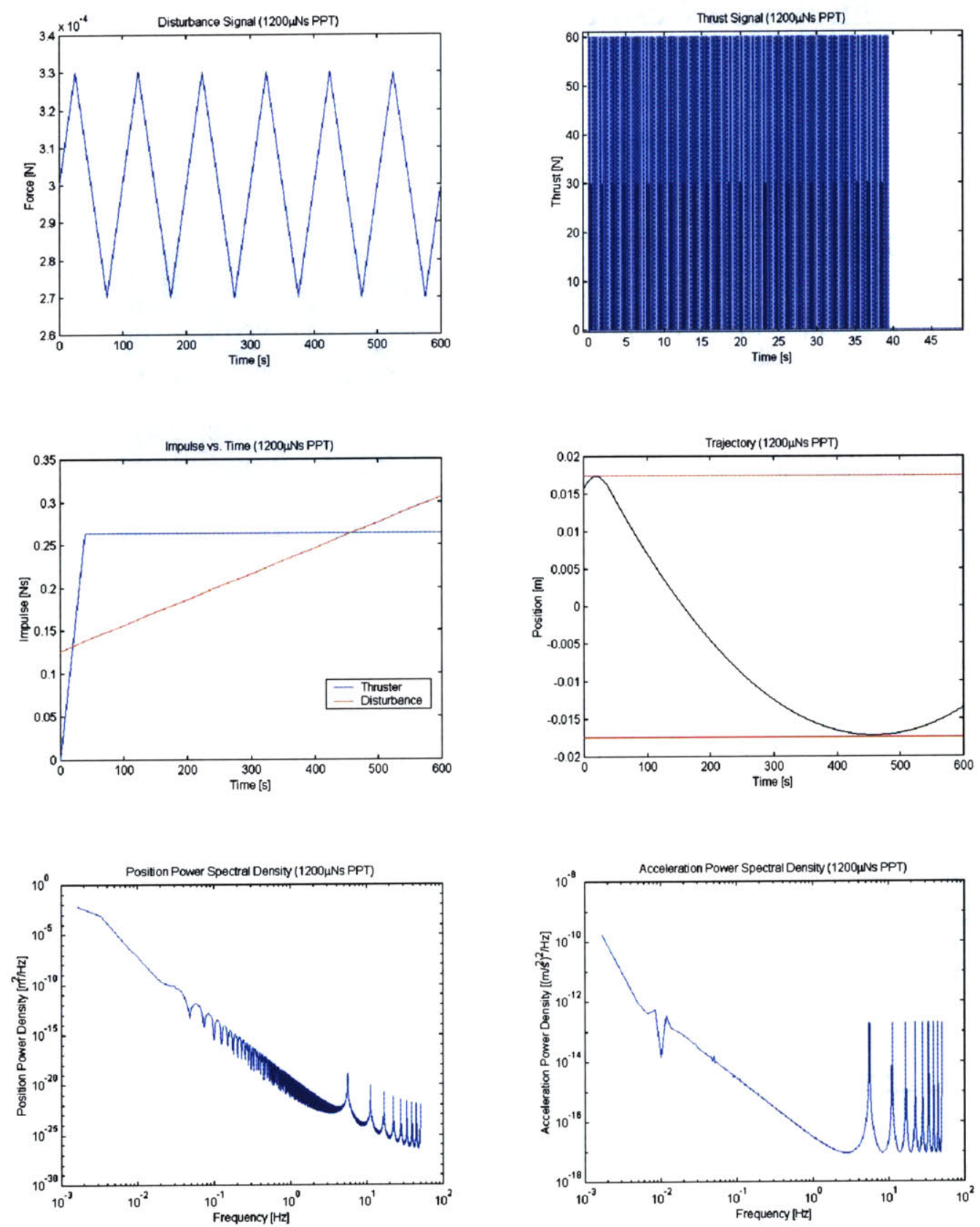

Figure B.10 Simulation results for the TPF combiner spacecraft using PPTs. 


\section{B.2.2 Colloid}
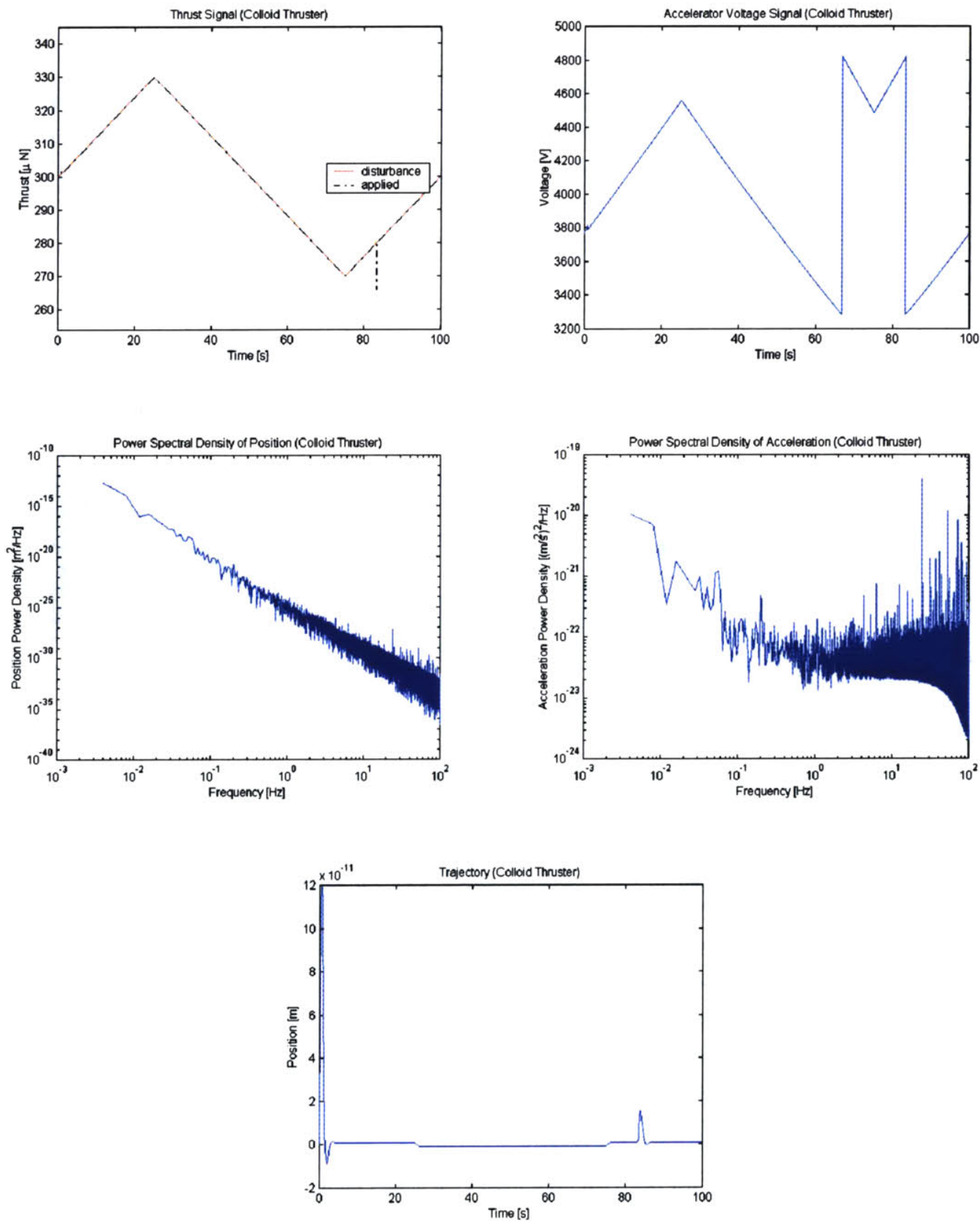

Figure B.11 Simulation results for the TPF collector spacecraft using colloid thrusters. 

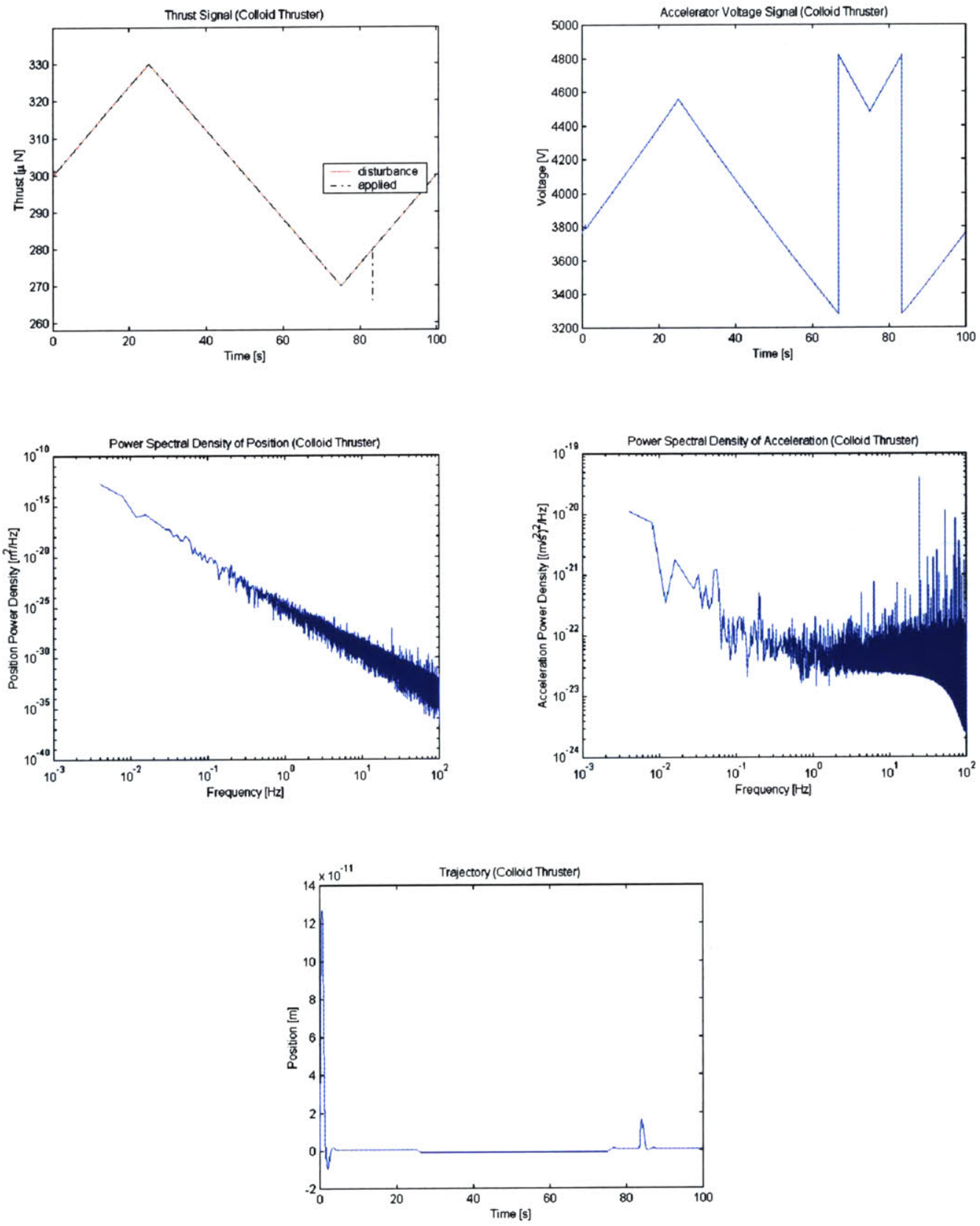

Figure B.12 Simulation results for the TPF combiner spacecraft using colloid thrusters. 


\section{B.2.3 FEEP}
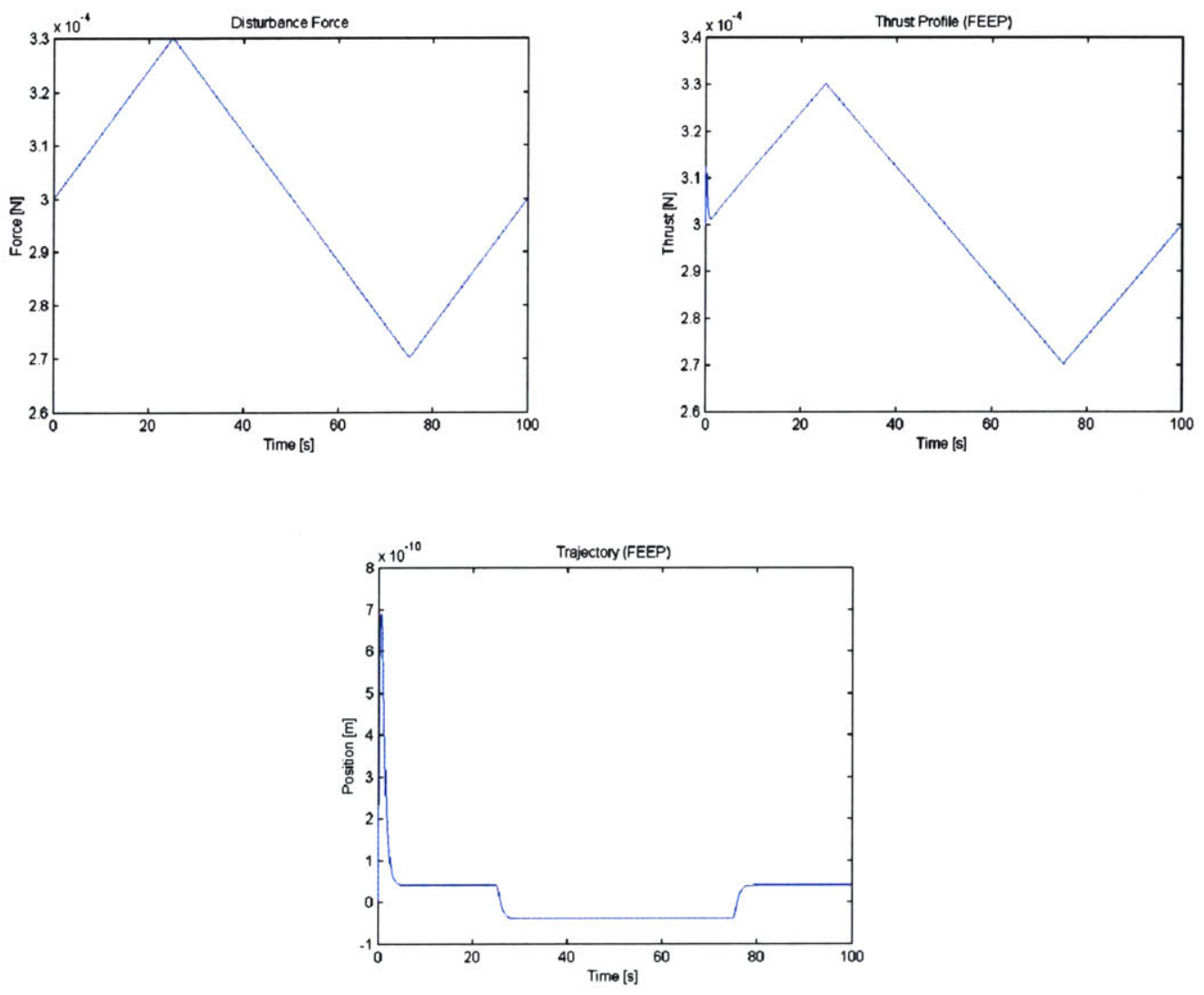

Figure B.13 Simulation results for the TPF collector spacecraft using a FEEP system.
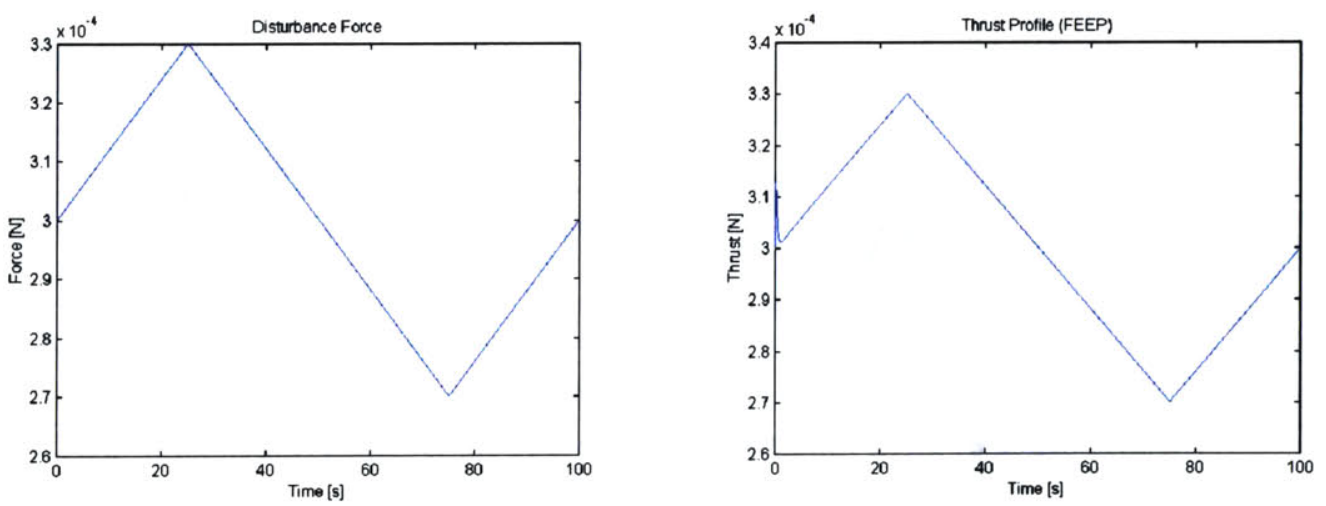

Figure B.14 Simulation results for the TPF combiner spacecraft using a FEEP system. 


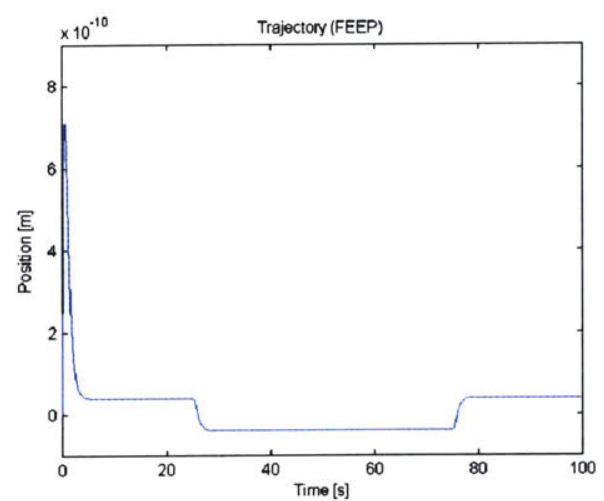

Figure B.14 Simulation results for the TPF combiner spacecraft using a FEEP system.

\section{B.2.4 Cold Gas}
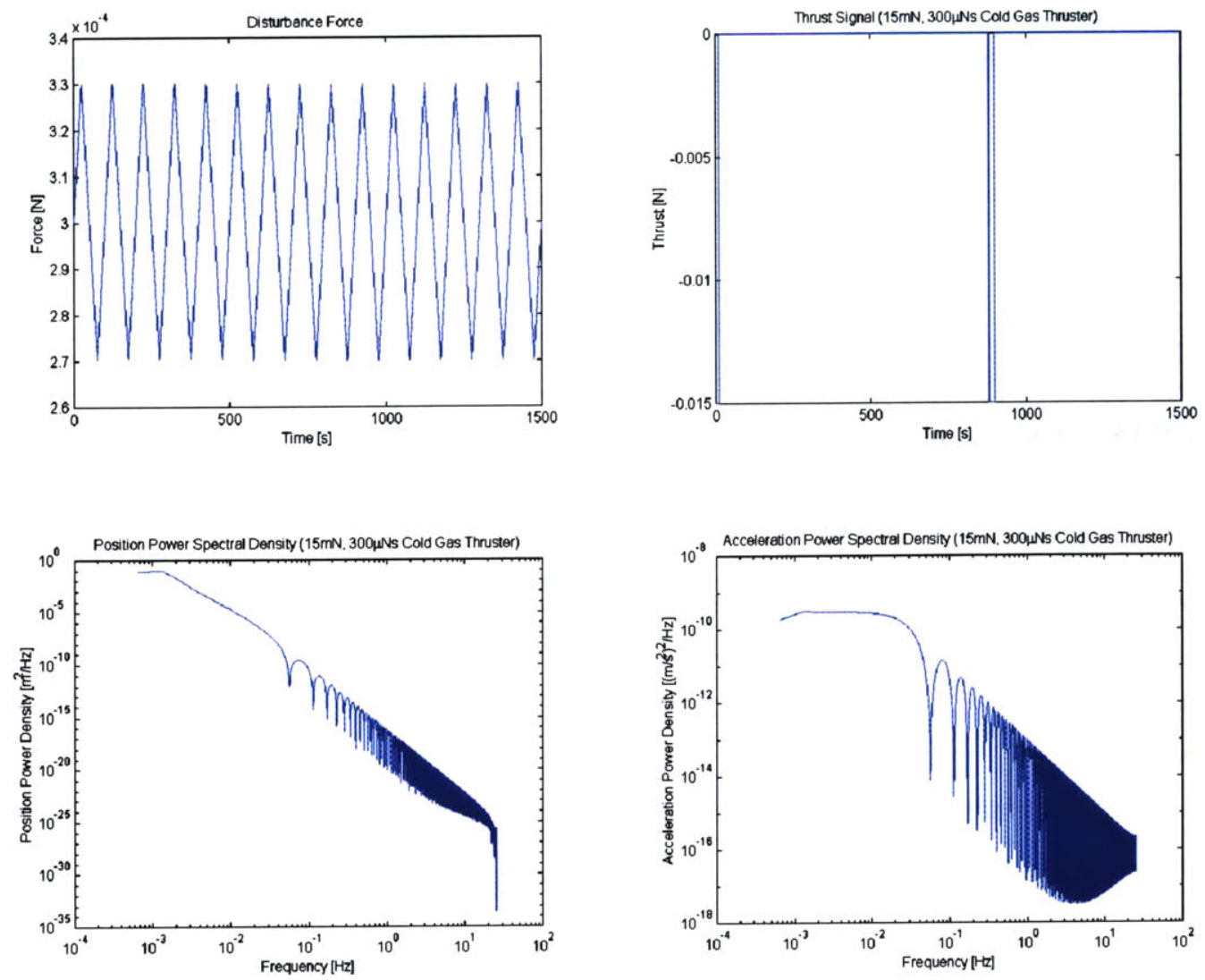

Figure B.15 Simulation results for the TPF collector spacecraft using cold gas thrusters. 


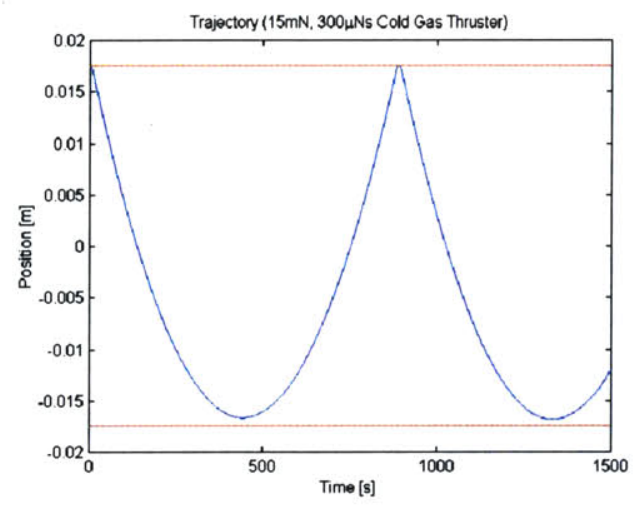

Figure B.15 Simulation results for the TPF collector spacecraft using cold gas thrusters.
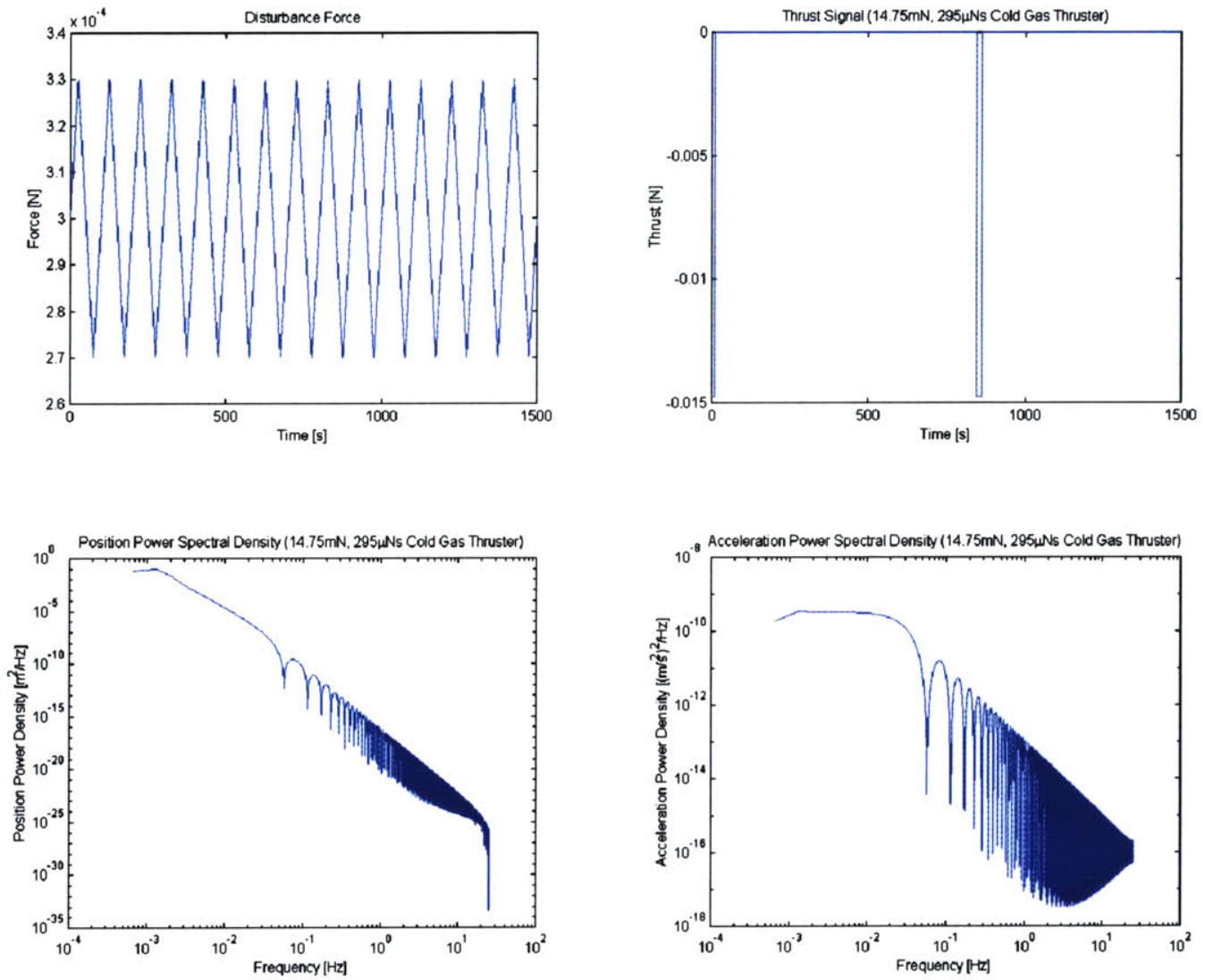

Figure B.16 Simulation results for the TPF combiner spacecraft using cold gas thrusters. 


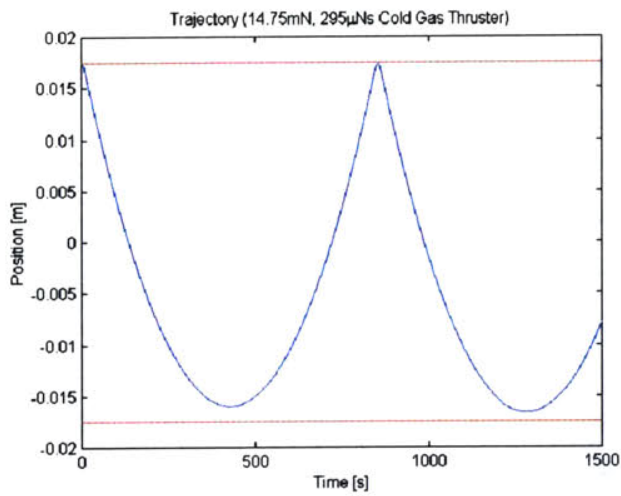

Figure B.16 Simulation results for the TPF combiner spacecraft using cold gas thrusters. 


\section{B.3 LISA Simulation Results}

\section{B.3.1 PPT}
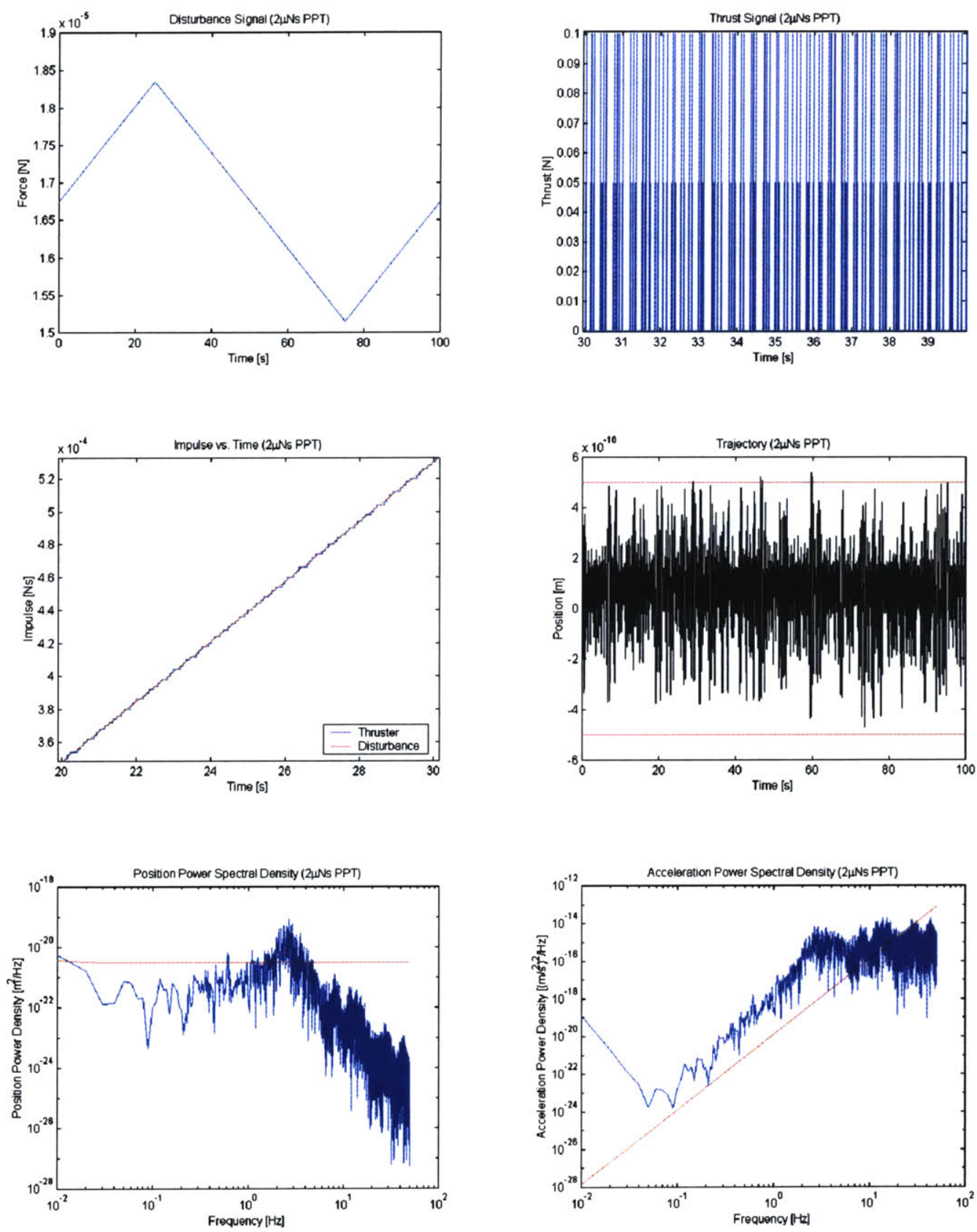

Figure B.17 Simulation results for the LISA spacecraft using PPTs. 


\section{B.3.2 Colloid}
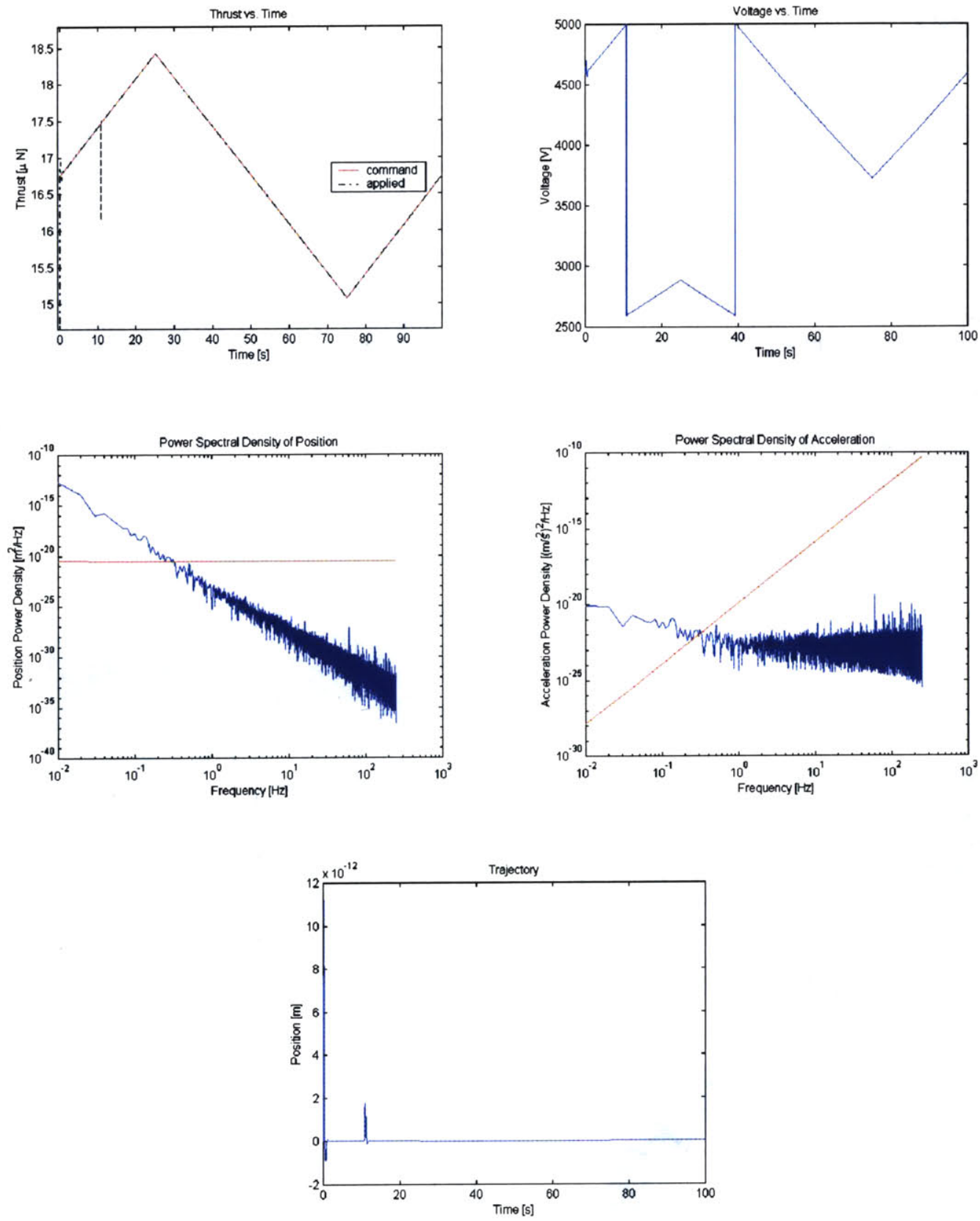

Figure B.18 Simulation results for the LISA spacecraft using colloid thrusters. 


\section{B.3.3 FEEP}
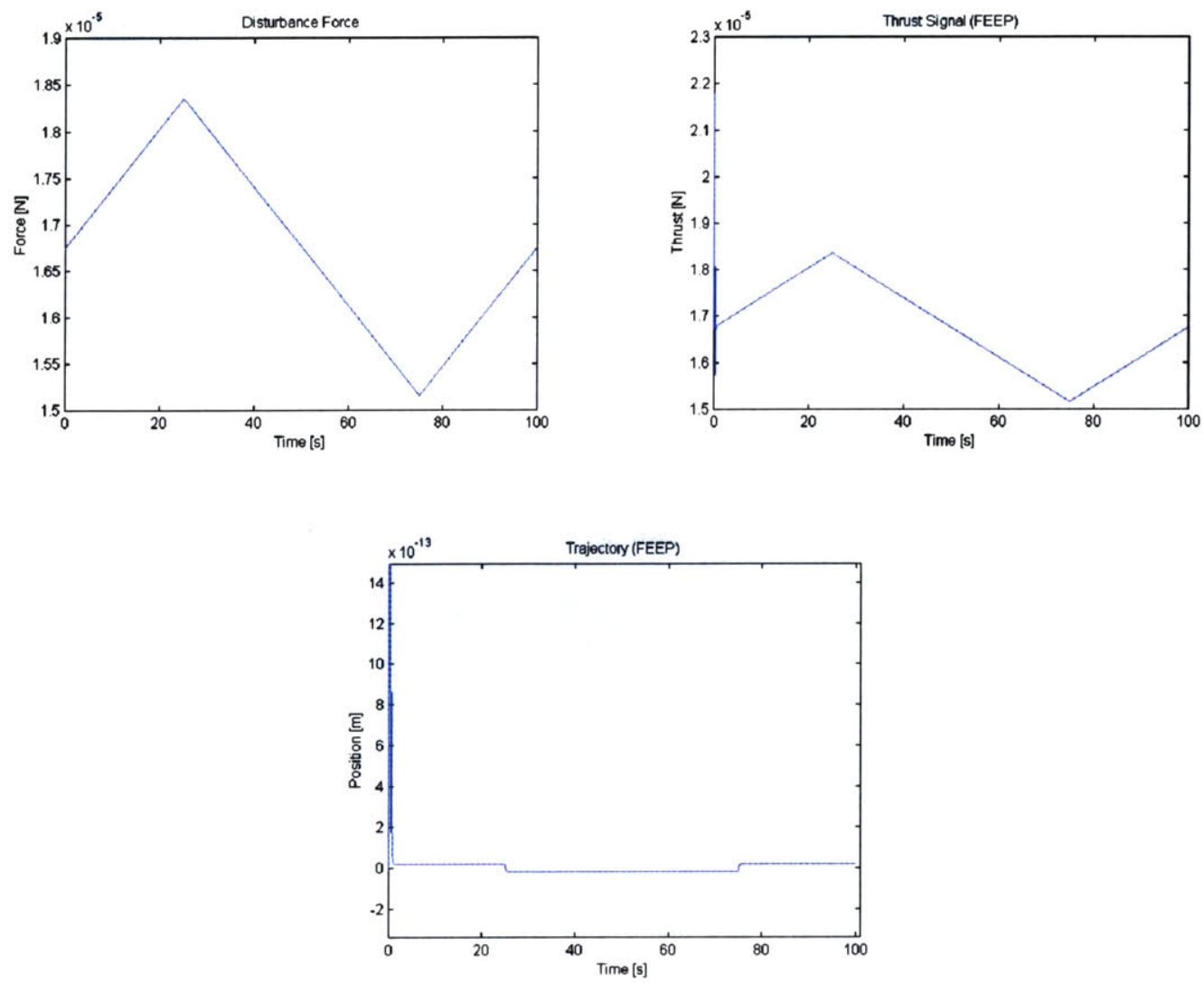

Figure B.19 Simulation results for the LISA spacecraft using a FEEP system.

\section{B.3.4 Cold Gas}
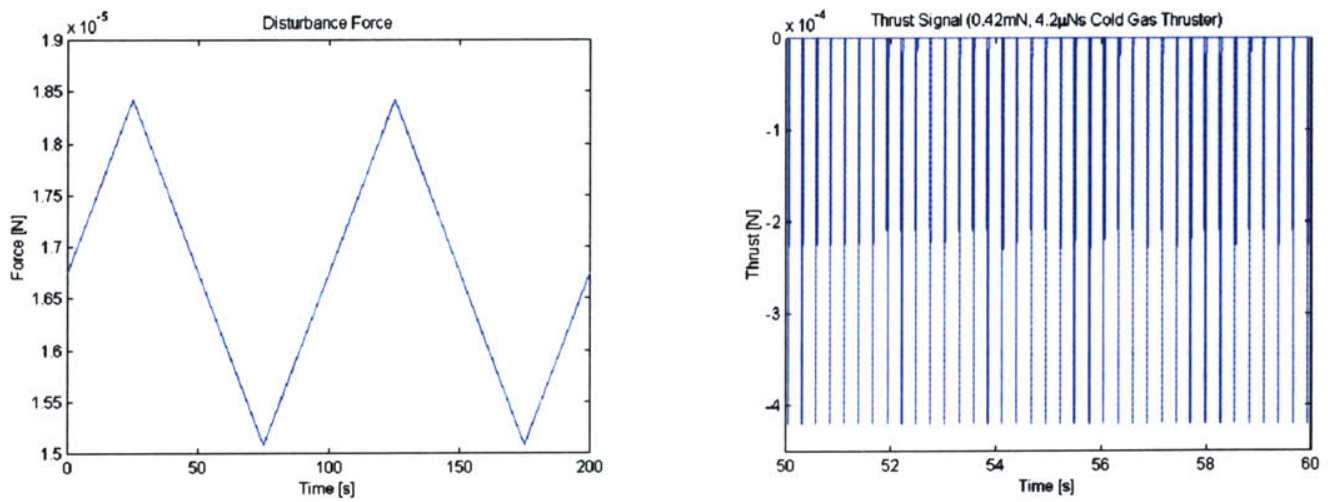

Figure B.20 Simulation results for the LISA spacecraft using cold gas thrusters. 

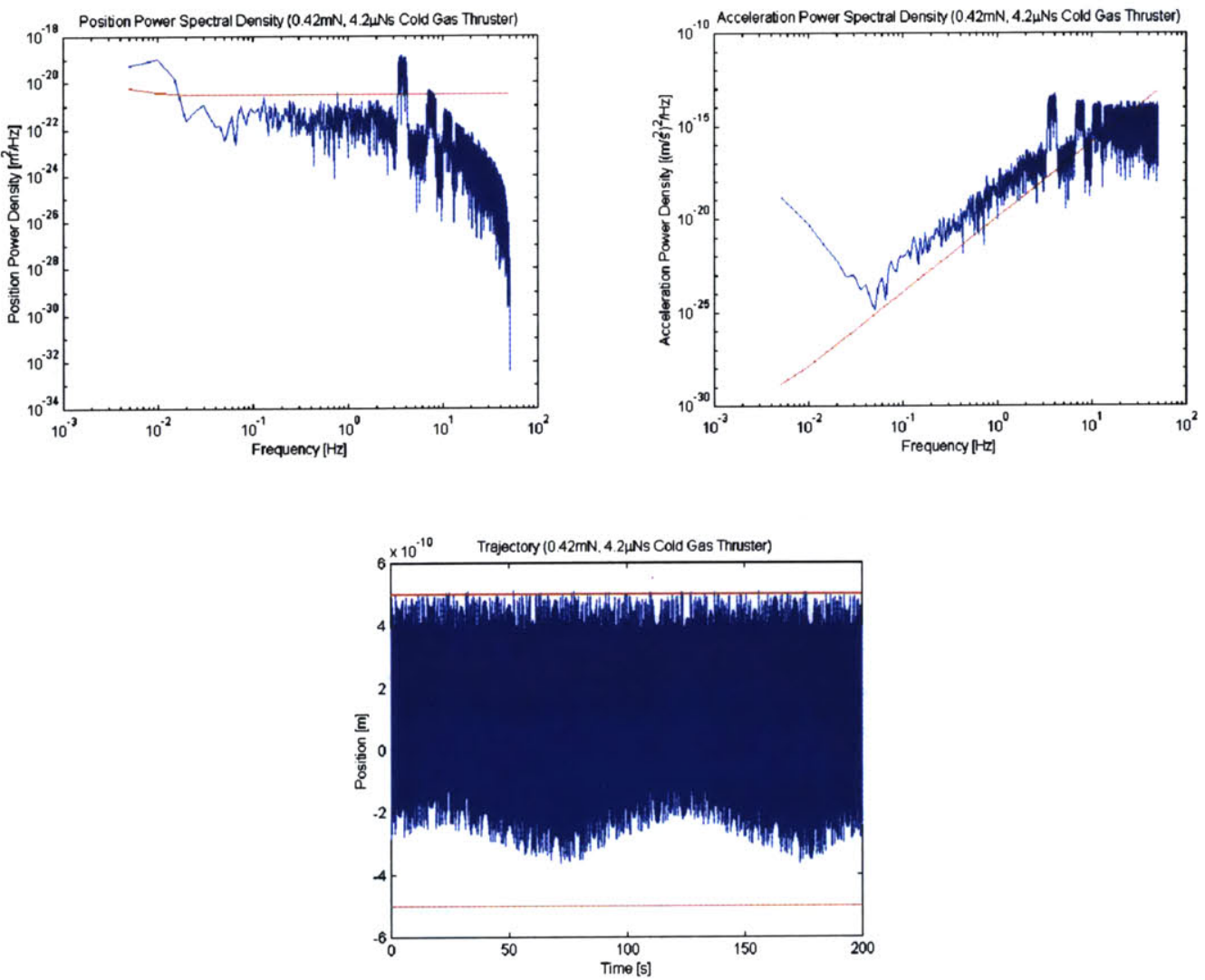

Figure B.20 Simulation results for the LISA spacecraft using cold gas thrusters. 


\section{B.4 MAXIM Simulation Results}

\section{B.4.1 PPT}
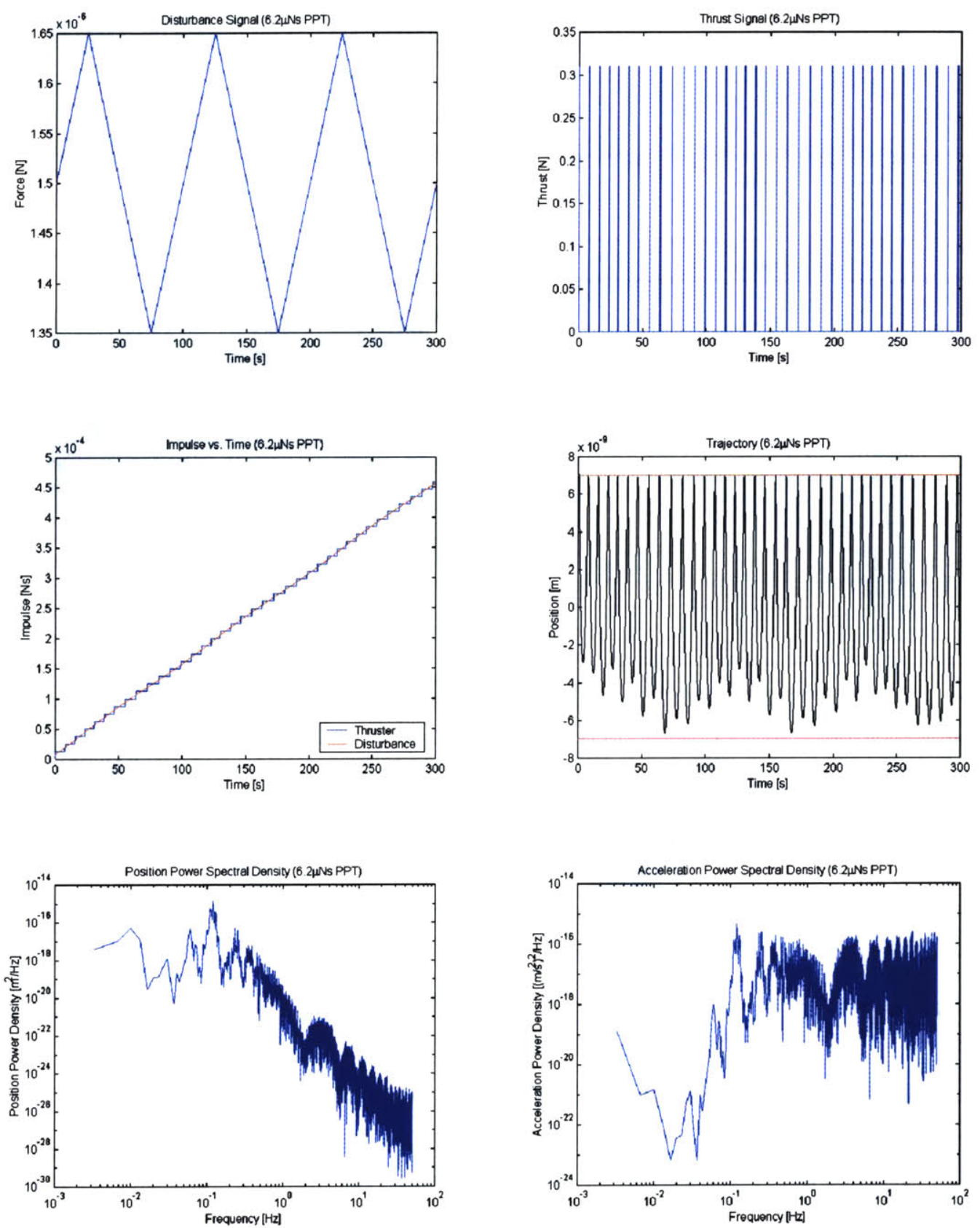

Figure B.21 Simulation results for the MAXIM collector spacecraft using PPTs. 

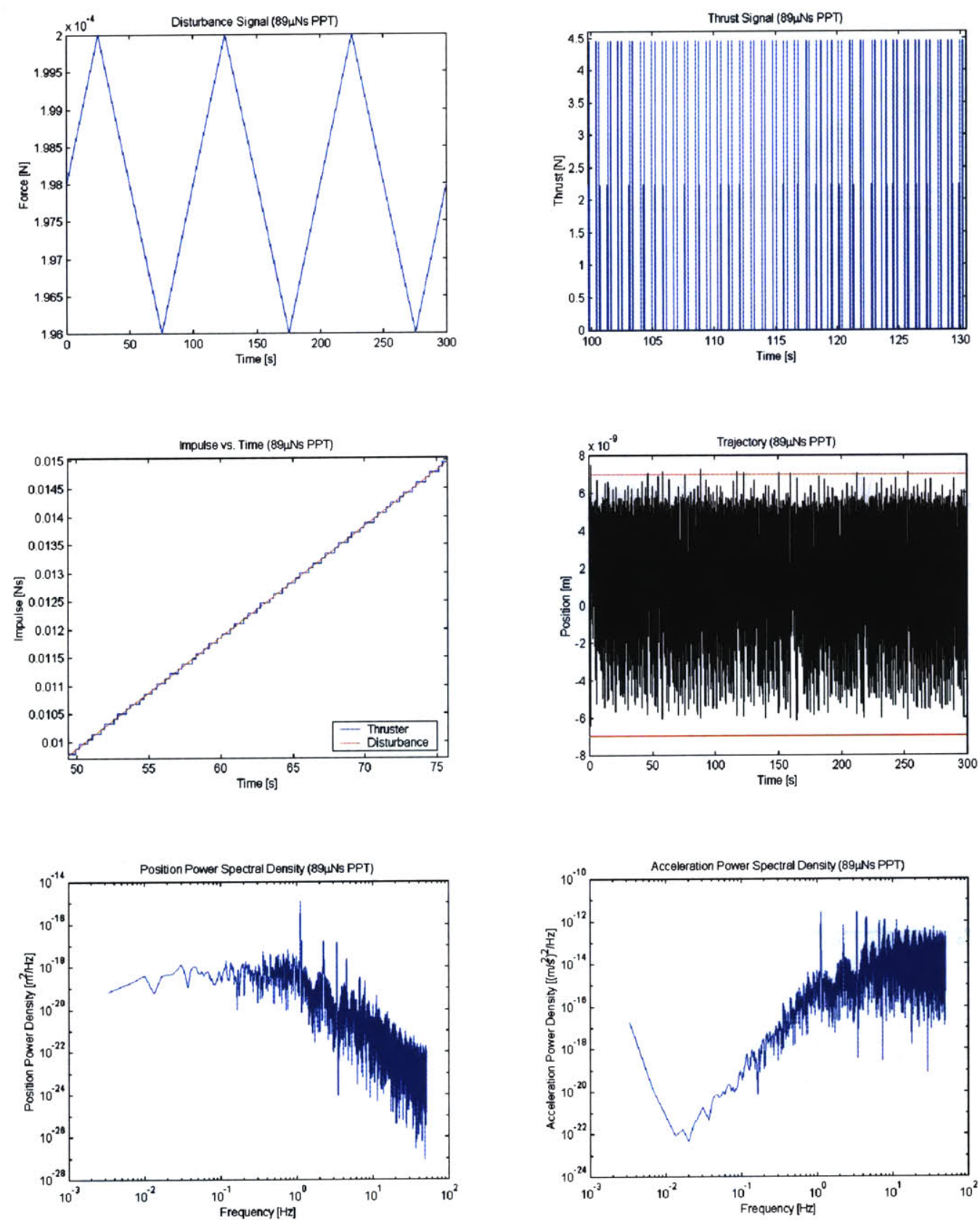

Figure B.22 Simulation results for the MAXIM detector spacecraft using PPTs. 


\section{B.4.2 Colloid}
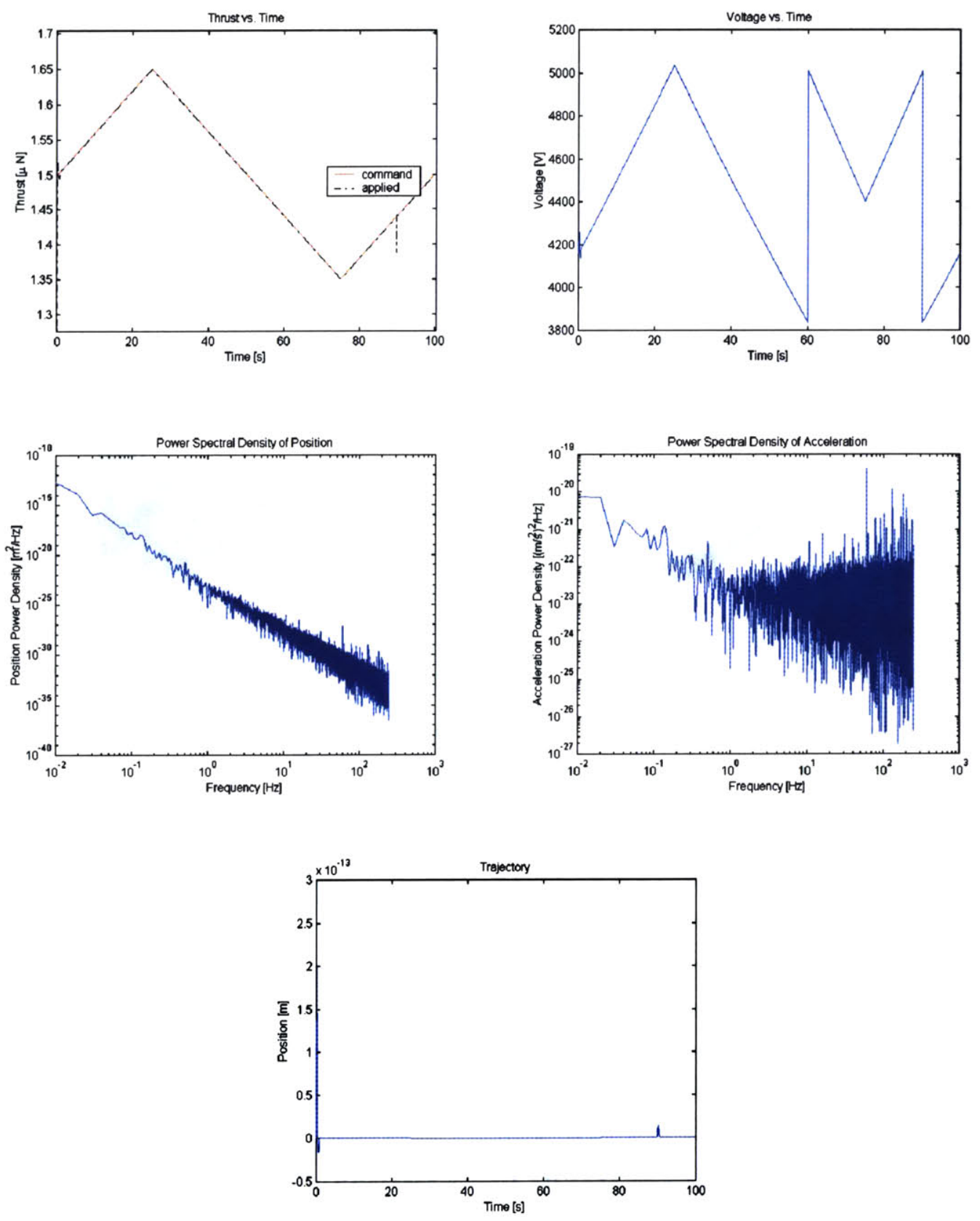

Figure B.23 Simulation results for the MAXIM collector spacecraft using colloid thrusters 

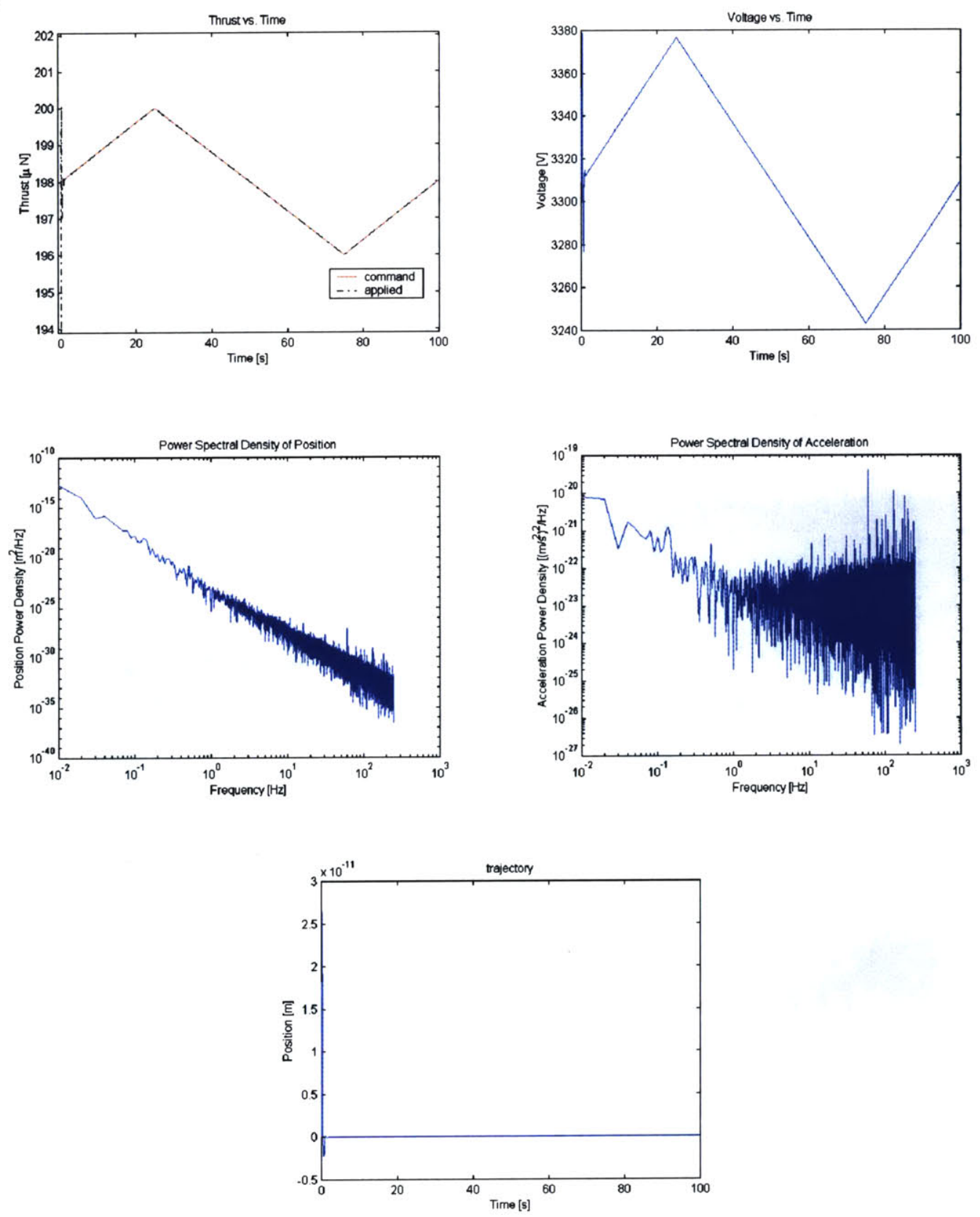

Figure B.24 Simulation results for the MAXIM detector spacecraft using colloid thrusters 


\section{B.4.3 FEEP}
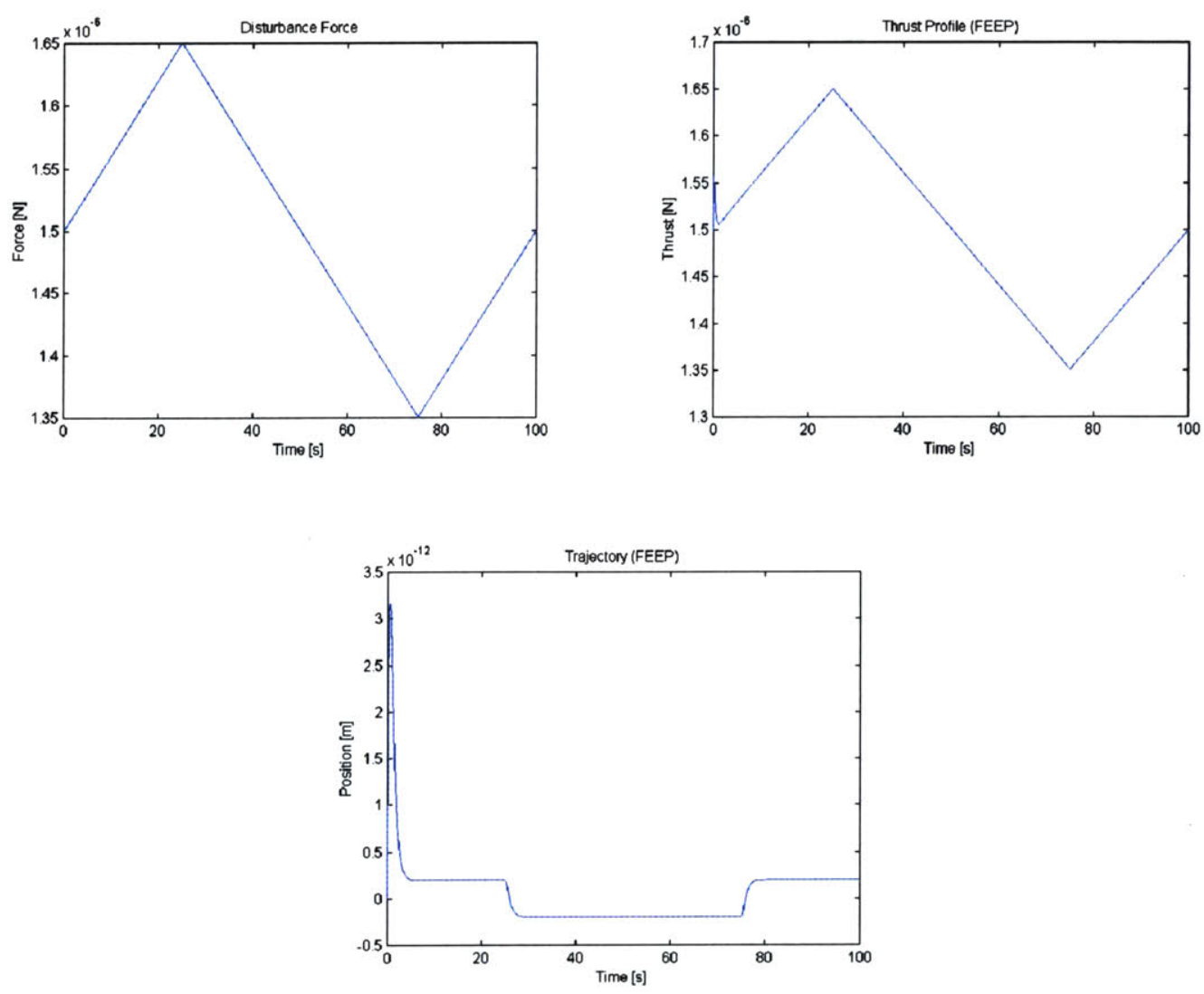

Figure B.25 Simulation results for the MAXIM collector spacecraft using a FEEP system.
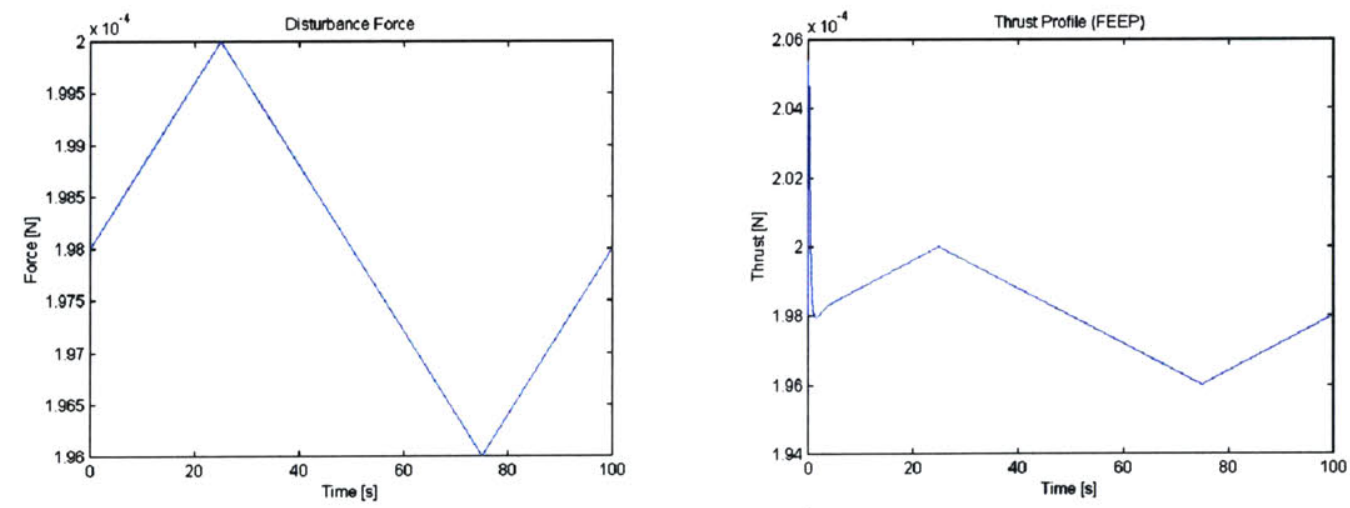

Figure B.26 Simulation results for the MAXIM detector spacecraft using a FEEP system. 


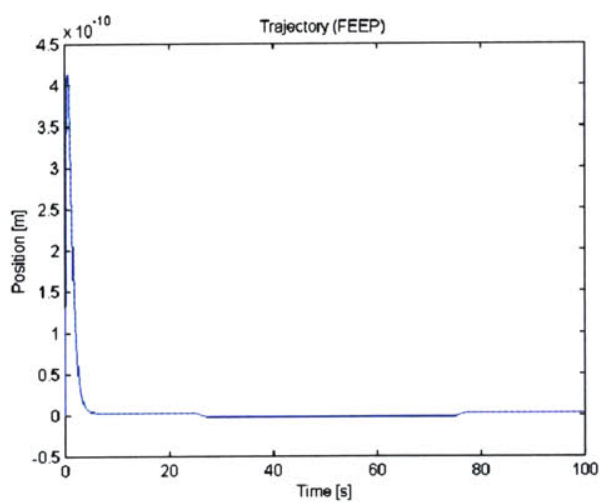

Figure B.26 Simulation results for the MAXIM detector spacecraft using a FEEP system.

\section{B.4.4 Cold Gas}
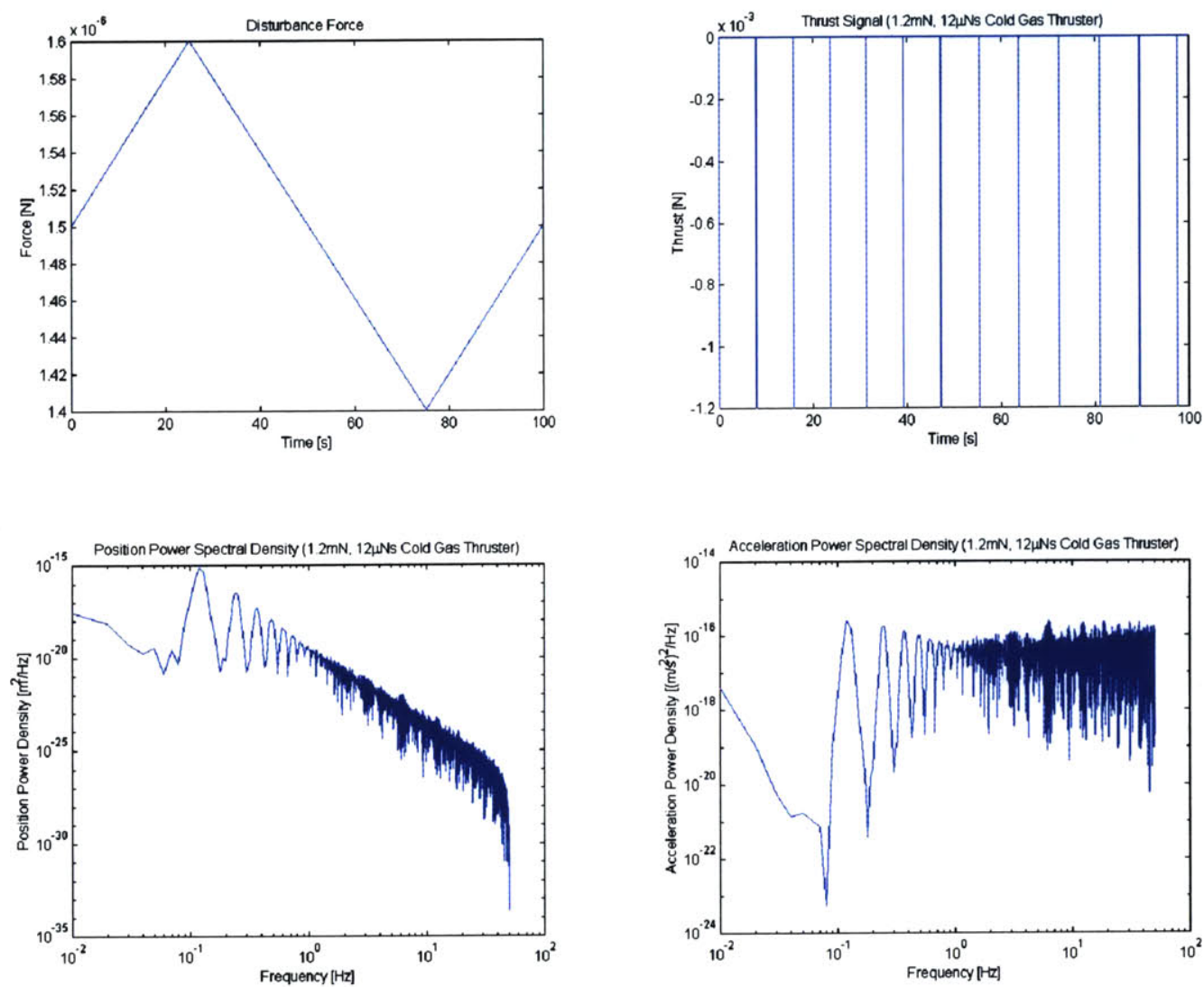

Figure B.27 Simulation results for the MAXIM collector spacecraft using cold gas thrusters. 


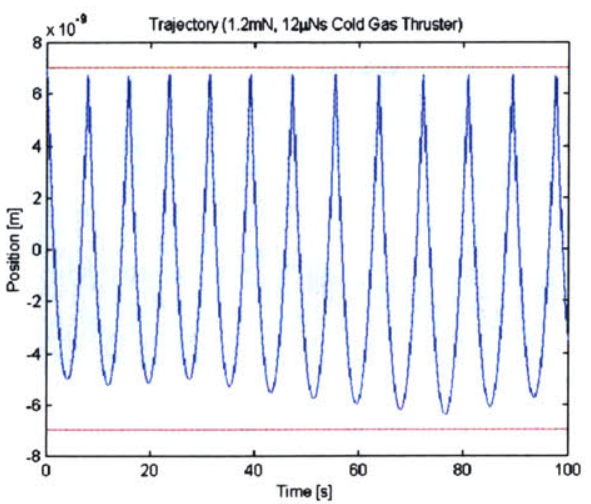

Figure B.27 Simulation results for the MAXIM collector spacecraft using cold gas thrusters.
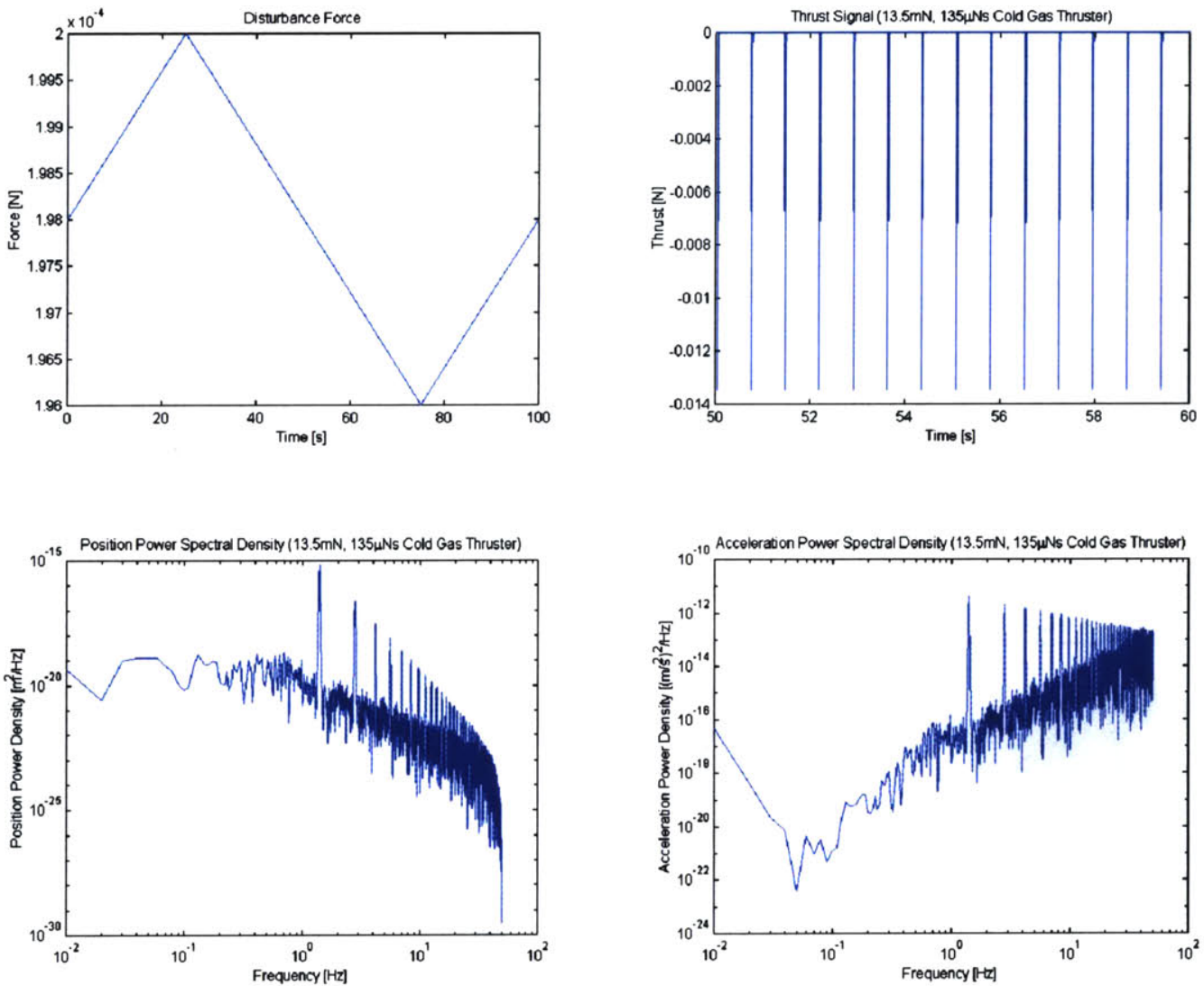

Figure B.28 Simulation results for the MAXIM detector spacecraft using cold gas thrusters. 


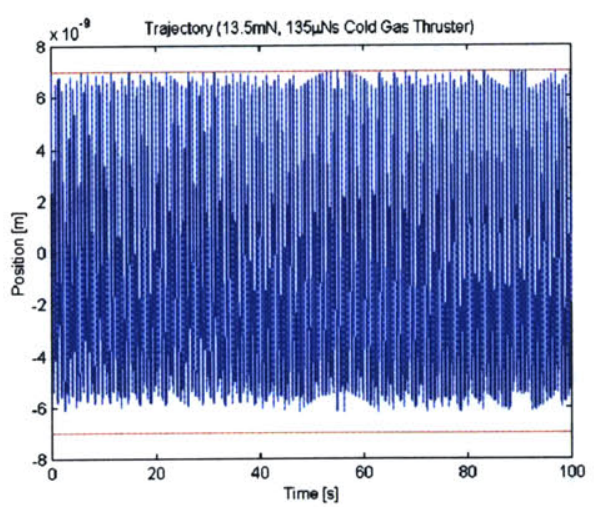

Figure B.28 Simulation results for the MAXIM detector spacecraft using cold gas thrusters. 


\section{B.5 SPECS Simulation Results}

\section{B.5.1 PPT}
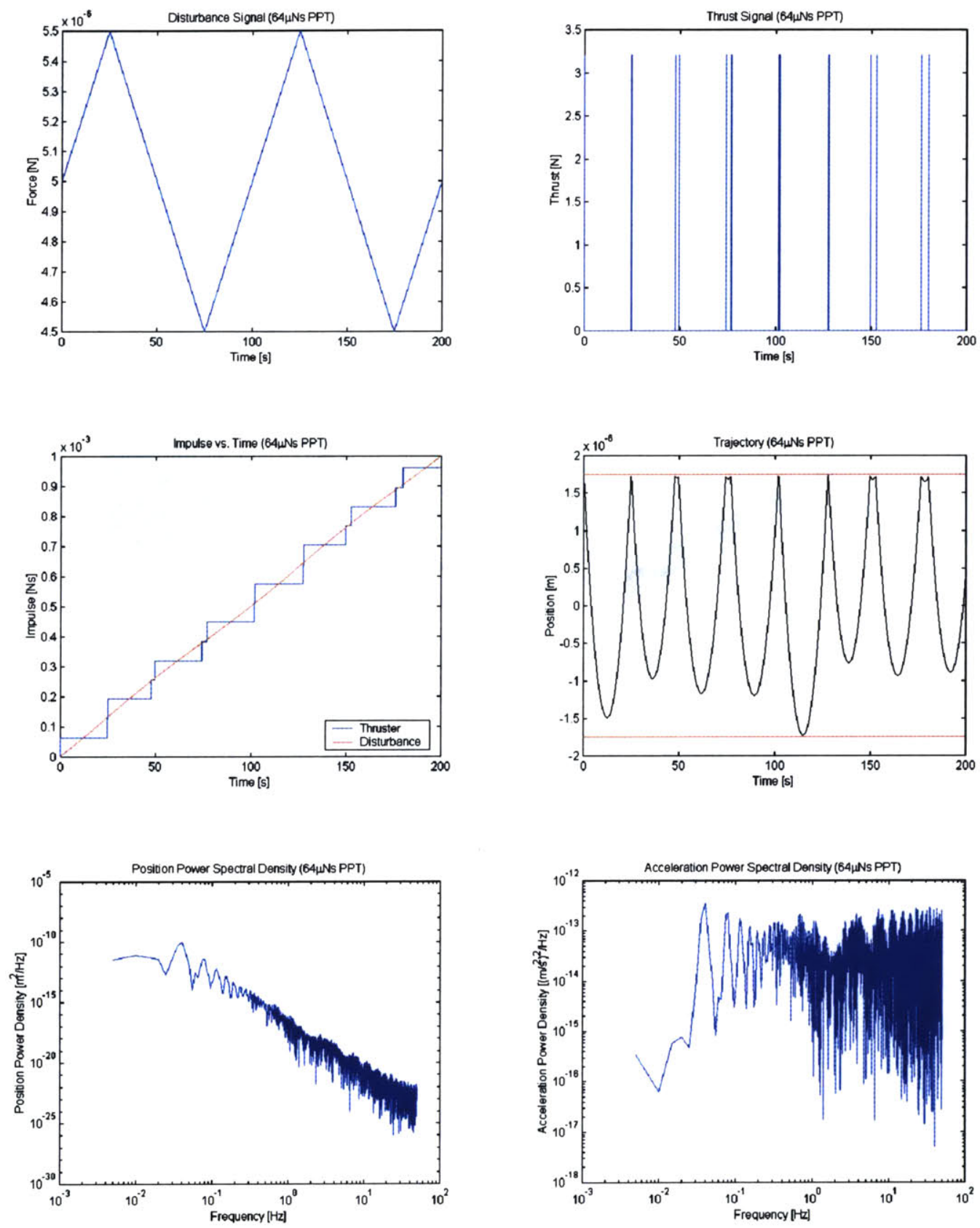

Figure B.29 Simulation results for the SPECS spacecraft using PPTs. 


\section{B.5.2 Colloid}
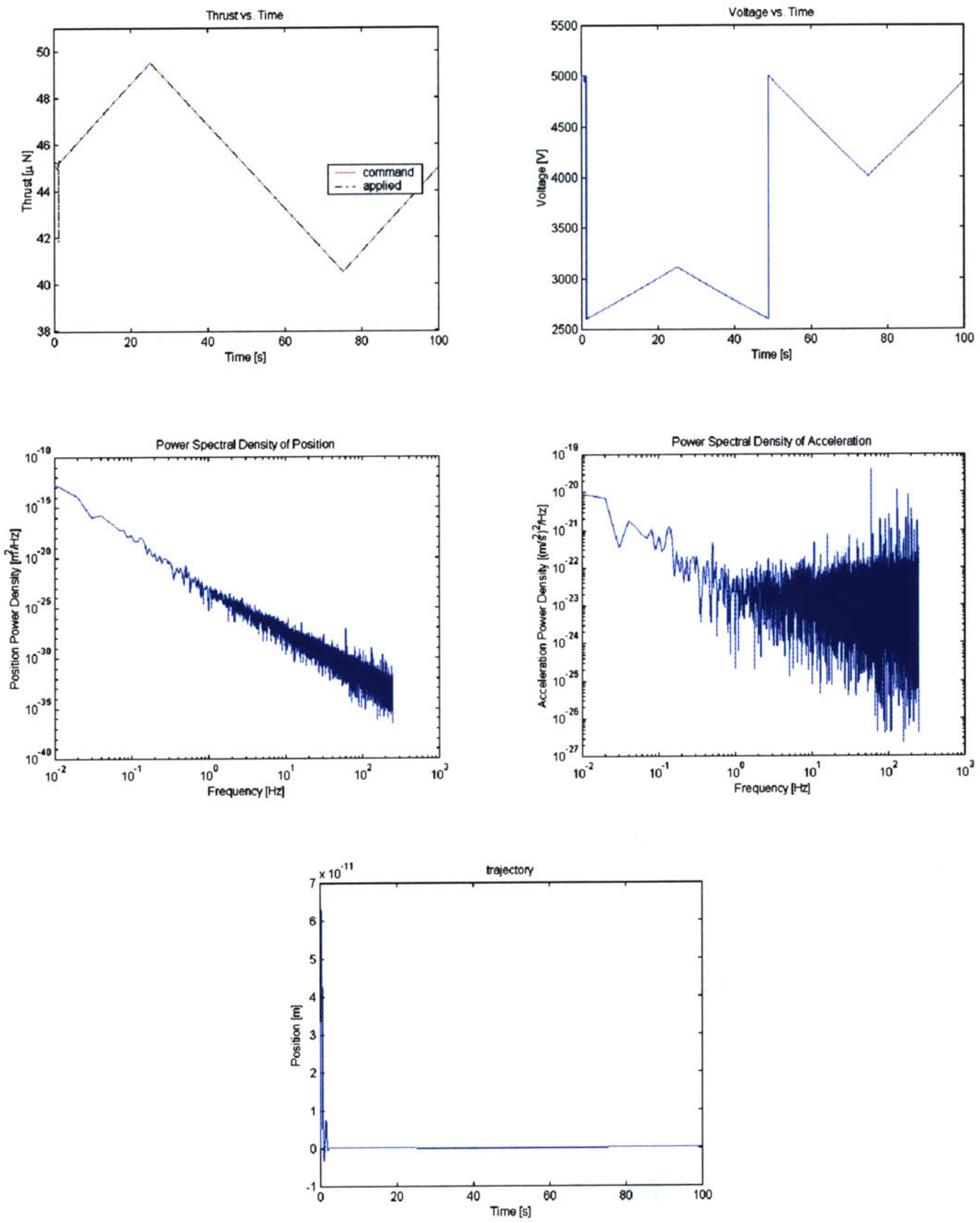

Figure B.30 Simulation results for the SPECS spacecraft using colloid thrusters. 


\section{B.5.3 FEEP}
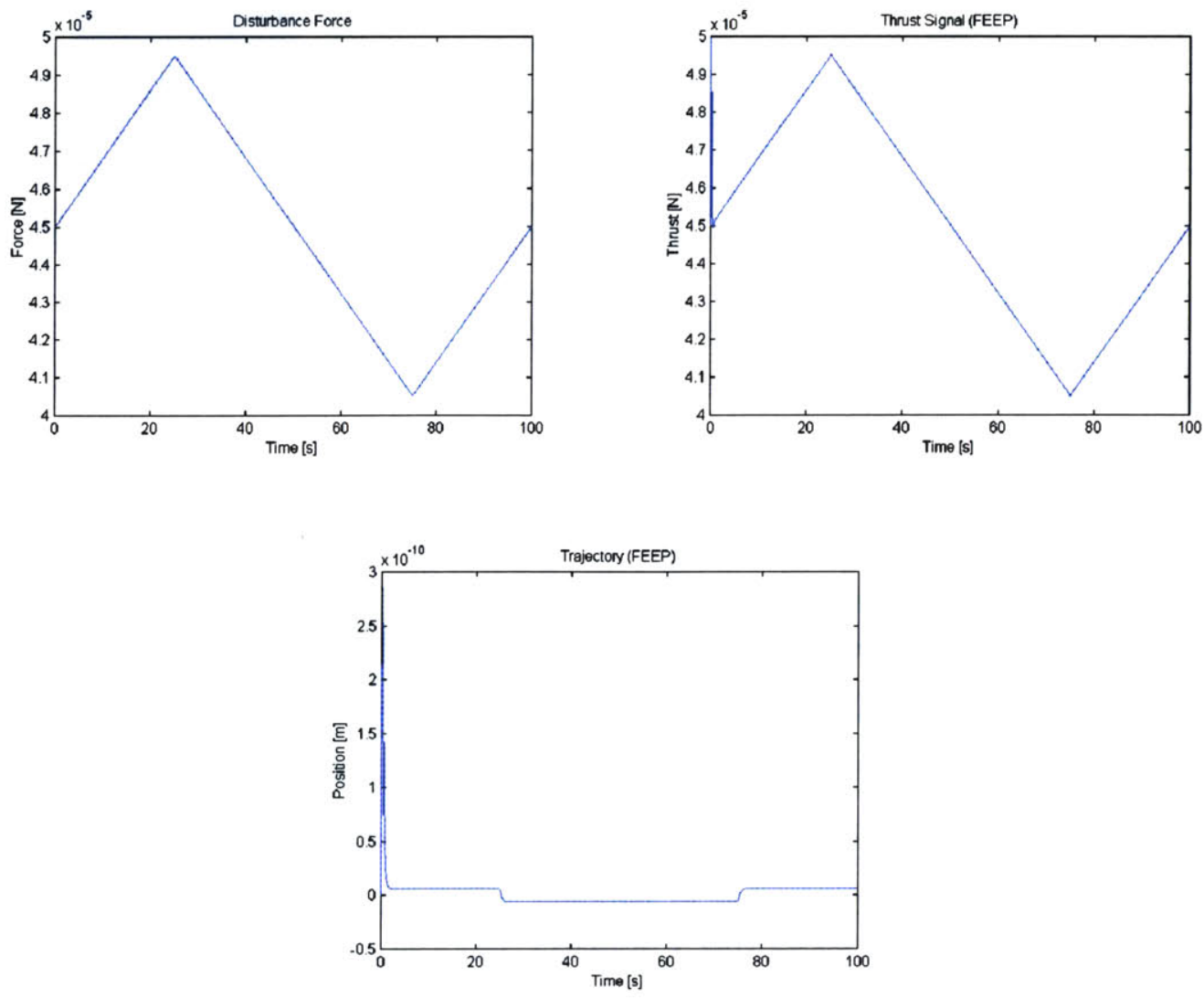

Figure B.31 Simulation results for the SPECS spacecraft using a FEEP system.

\section{B.5.4 Cold Gas}
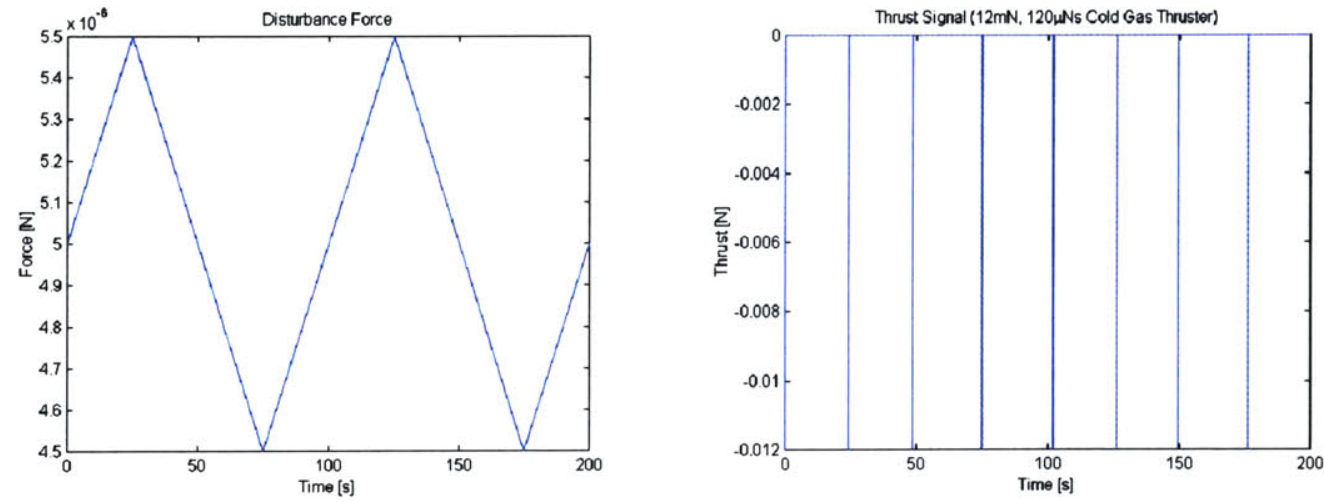

Figure B.32 Simulation results for the SPECS spacecraft using cold gas thrusters. 

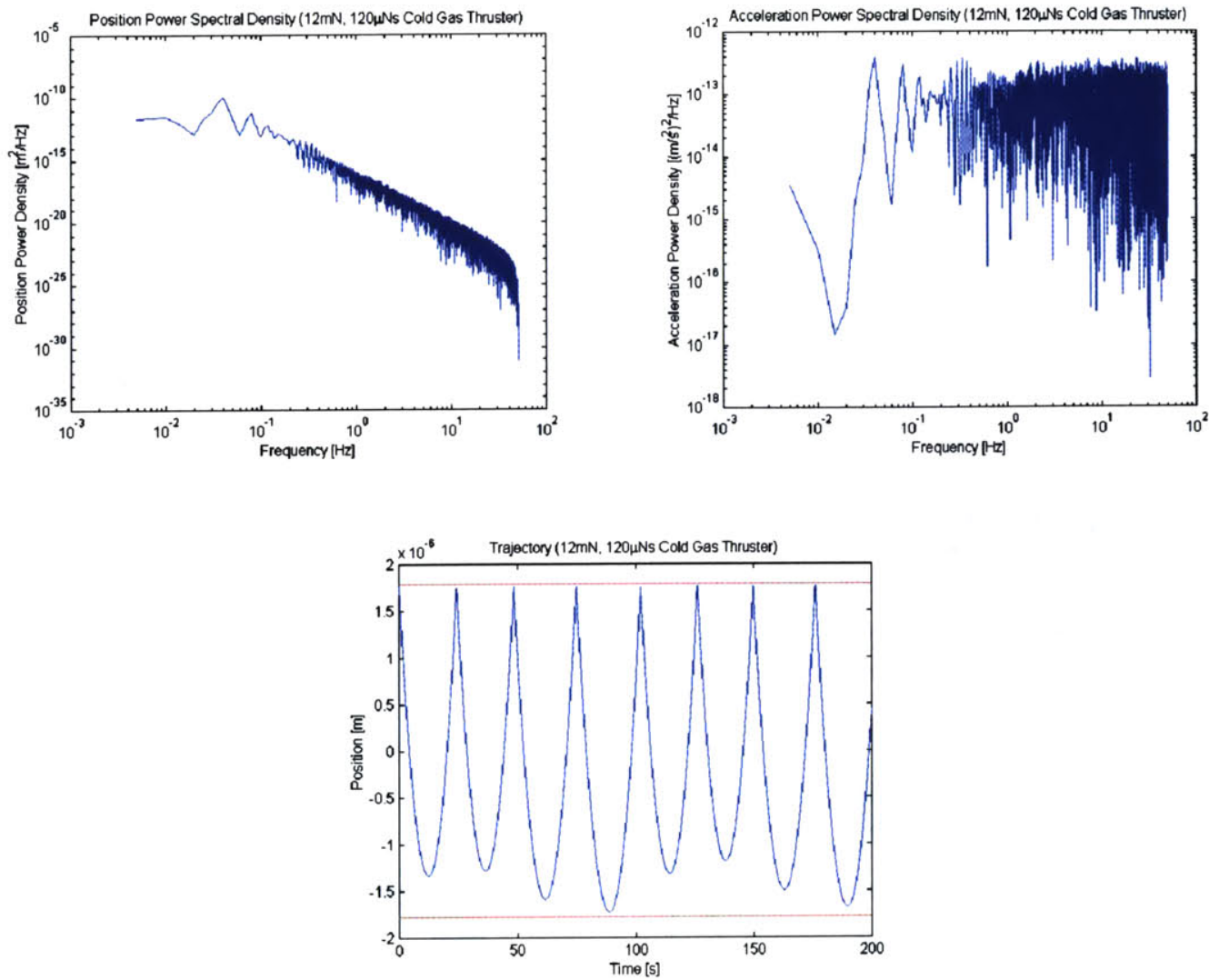

Figure B.32 Simulation results for the SPECS spacecraft using cold gas thrusters. 


\section{Appendix C}

\section{DESIGN EQUATIONS}

\section{C.1 PPT Design Equations}

The design methodology discussed in Section 5.1.1 is carried out using the following set of equations, which relate the salient variables of the PPT propulsion system to each other. All quantities are in MKS units; lengths are in meters, masses are in kilograms and times are in seconds resulting in: forces in Newtons, energies in Joules, powers in Watts, frequencies in Hertz, currents in Amperes, voltages in Volts, etcetera.

The equation for the energy storage size of the discharge capacitor is given in terms of the impulse bit of the thruster by the relationship:

$$
E_{o}=\frac{\left(10^{6} I_{b i t}+142\right)}{23.2} \text {, }
$$

which is taken from [Guman, 1975]. Simply, by multiplying the capacitor energy by the maximum pulse rate of the thruster, the maximum average power input to the thruster is found as

$$
P_{\text {max }}=E_{o} P R F_{\text {max }} .
$$

The following two equations provide information about the efficiency with which the PPT uses propellant to provide thrust. Equation C.3 [Guman, 1975] does not apply to the regime that the micro-PPT designs (those with $\mathrm{I}_{\text {bit }} \sim 5 \mu \mathrm{Ns}$ ) lie in, so supplementary data 
[Gulczinski, 2000] was used to obtain a reasonable value for the $\mathrm{m}_{\text {shot }}$, which was then used in Equation C. 4 to determine a corresponding $\mathrm{I}_{\text {bit }}$.

$$
\begin{gathered}
I_{s p}=\frac{2500 E_{o}^{1.6}}{10^{6} I_{b i t}} \\
m_{s h o t}=\frac{I_{b i t}}{I_{s p} g_{o}}
\end{gathered}
$$

The maximum current demanded from the spacecraft by the thruster system is modeled by a very straightforward relationship,

$$
I_{\max }=\frac{E_{o} P R F_{\max }}{\mathrm{V}_{\mathrm{s} / \mathrm{c}} \eta}
$$

which equates the average power the thruster needs to consume to the average power the spacecraft must produce for it with a power transmission efficiency factor (assumed to be 0.85 ) and an assumed spacecraft voltage of $28 \mathrm{~V}$. The following three equations for the mass of various propulsion system components comes from the same paper by Guman that is the source for Equation C.1 [Guman, 1975] and is based on experimental data.

$$
\begin{gathered}
m_{c}=\frac{E_{o}}{88} \\
m_{p p u}=0.5+5 \times 10^{-3} P_{\text {max }} \\
m_{d i c}+m_{s}=0.73
\end{gathered}
$$

Some of these relationships were supplemented or modified for use with the micro-PPT designs, which rely on point design data from [Gulczinski, 2000]. The mass of propellant used by a PPT is determined by the following equation, which just multiplies the number of pulses required to meet the mission $\Delta v$ requirement by the mass ablated per pulse: 


$$
m_{\text {prop }}=\frac{m_{\mathrm{s} / \mathrm{c}} m_{\text {shot }} \Delta \mathrm{v}}{I_{\text {bit }}}
$$

A simple sum of the various component masses multiplied by the number of thrusters per spacecraft gives a total mass for the propulsion system (per spacecraft). It is assumed, however, that there need only be one power processing unit for every two thrusters while retaining a high level of redundancy, because situations requiring half or more of the thrusters on a spacecraft to thrust simultaneously are unreasonable.

$$
m_{\text {total }}=N_{\frac{\text { thrusters }}{\mathrm{s} / \mathrm{c}}}\left(m_{c}+m_{\text {dic }}+m_{s}+\frac{m_{p p u}}{2}\right)+m_{\text {prop }}
$$

\section{C.2 Colloid Thruster Design Equations}

The design methodology discussed in Section 5.1.2 is carried out using the following set of equations, which relate the salient variables of the colloid thruster propulsion system to each other. All quantities are in MKS units; lengths are in meters, masses are in kilograms and times are in seconds resulting in: forces in Newtons, energies in Joules, powers in Watts, frequencies in Hertz, currents in Amperes, voltages in Volts, etcetera.

Equation C.11 provides a relationship between the volume flow rate of the propellant in a single needle, $Q$, and the nominal operating voltage for that needle. It is based on a pressure balance between the surface tension induced pressure, propellant tank applied pressure, laminar flow associated pressure drop and electrostatic pressure. It is an approximation, with lack of precision in the setting of some scale factors of order one, but it is anchored by reasonable values (based on observation and analysis) for the minimum volume flow rate, $Q_{\min }$, and the minimum operating/starting voltage, $\mathrm{V}_{\min }$.

$$
\mathrm{V}_{\text {nominal }}=\sqrt{\frac{64 \mu L\left(Q-Q_{\min }\right)+\varepsilon_{o} d^{2} \mathrm{~V}_{\text {min }}^{2}}{\varepsilon_{o} d^{2}}}
$$


The current emitted by each needle is expressed in Equation C.12, a result first formulated by Fernandez de la Mora empirically [Fernandez de la Mora, 1994]. It is only dependent on the volume flow rate and the fluid properties.

$$
I_{N}=f(\varepsilon) \sqrt{\frac{\gamma_{\mathrm{st}} \mathrm{k} Q}{\varepsilon}}
$$

In addition, the charge to mass ratio of the, assumed monodisperse, droplets is determined from the volume flow rate, $Q$, and the other fluid properties. The following expression comes from equating the charge to mass ratio to the current to mass flow rate ratio, which basically just enforces monodispersity.

$$
\frac{q_{d}}{m_{d}}=\frac{f(\varepsilon)}{\rho_{\text {prop }}} \sqrt{\frac{\gamma_{\mathrm{st}} \mathrm{\kappa}}{Q \varepsilon}}
$$

The nominal thrust provided by each needle is then given by the simple expression in Equation C.14, which is just the product of mass flow rate of propellant, $\rho_{\text {prop }} Q$, and the exhaust velocity, which can be varied by changing the accelerating voltage, $V_{i}$.

$$
F_{N}=\rho_{\text {prop }} Q \sqrt{2 \frac{q_{d}}{m_{d}} \mathrm{~V}_{\text {nominal }}}
$$

The specific impulse, used later in Equation C.19 to compute the propellant mass for the mission, is the same exhaust velocity used in the thrust equation, C.14, but normalized by the earth gravitational constant. In this case we are assuming a nominal accelerating voltage, but $I_{\mathrm{sp}}$ will vary with applied accelerating voltage, despite the fact that the extracting voltage remains constant.

$$
I_{\mathrm{sp}_{\mathrm{avg}}}=\frac{1}{g_{o}} \sqrt{2 \frac{q_{d}}{m_{d}} \mathrm{~V}_{\text {nominal }}}
$$

As seen in Equation C.14, thrust scales as the operating voltage to the one half power. So, the number of needles required to achieve a maximum thrust, $F_{\max }$, is the maximum thrust 
desired, divided by the maximum thrust that can be provided by a single needle. The square root of the ratio of the maximum voltage to the nominal voltage gives the factor by which the nominal needle thrust is augmented. When multiplied by the nominal needle thrust, it gives the maximum thrust from a single needle. Equation C.16 expresses the number needles necessary for each thruster in these terms.

$$
N_{N}=\frac{F_{\text {max }}}{F_{N}} \sqrt{\frac{\mathrm{V}_{\text {nominal }}}{\mathrm{V}_{\text {max }}}}
$$

The maximum power draw of a single thruster unit is then given simply by the product of the current and voltage delivered by a single needle divided by the efficiency of power conversion (assumed to be in the range of 0.7 and 0.8 ) and multiplied by the number of needles in the thruster array, as computed in Equation C.17. In order to determine the power for other regimes of operation it is necessary to determine how many needles and with what accelerating voltage are required for the desired thrust.

$$
P_{\text {max }}=\frac{I_{N} N_{N} \mathrm{~V}_{\text {max }}}{\eta}
$$

The power required for the neutralizer, which prevents the spacecraft from building up a charge, is an estimation based on data from a space-proven PHILIPS Ba-impregnated cathode discussed by Tajmar [Tajmar, 2000].

$$
P_{\text {neutral }}=500 N_{N} I_{N}
$$

The propellant mass for the colloid thruster is estimated by the conventional rocket equation, where the $\mathrm{I}_{\mathrm{sp}}$ is based on the an approximated mean value for the mission. The exact $I_{\text {sp }}$ may not be able to be pinned down because it changes with changes in the thrust. Without precise information about the schedule of thrusting for a mission it can only be estimated. 


$$
m_{\text {prop }}=m_{\mathrm{s} / \mathrm{c}}\left(1-e^{-\left(\frac{\Delta \mathrm{v}}{I_{s p g_{0}}}\right)}\right)
$$

The masses for the various components of the colloid thruster come from extrapolations of a design for a $20 \mathrm{mN}$ thruster unit, using conventional manufacturing techniques, drawn up by Hruby at the Busek Company, Inc. [Hruby, 2000]. The mass for the structure, $m_{s}$, has been modified to account for MEMS microfabrication of the needles, electrodes and propellant reservoir. This information is based on preliminary numbers from an ongoing study, by Luis Velasquez, in the Space Propulsion Laboratory at MIT.

$$
\begin{gathered}
m_{s}=1.1\left(0.3+0.5 \times 10^{-5} N_{N}\right) \\
m_{p p u}=0.163 P_{\text {max }}+7.3 \times 10^{-2} \\
m_{\text {neutral }}=3 \times 10^{-2} \\
m_{\text {feed }}=1.2\left(0.33+m_{\text {prop }}\right)
\end{gathered}
$$

Note that the mass of the feed system, $m_{\text {feed }}$, in Equation C.23 includes the mass of the propellant, so that in the final mass summation only $m_{\text {feed }}$ appears, $m_{\text {prop }}$ does not. The maximum total power for a single thruster array is simply the maximum power required to operate the thrusters and the neutralizer.

$$
\text { Maximum } P_{\text {total }}=P_{\text {neutral }}+P_{\text {max }}
$$

The total mass for a single spacecraft is then sum of the components multiplied by the number of thrusters needed. However, the same assumption that only half as many power processing units as thrusters are necessary, as was made for the PPT and will be made for the FEEP, is made. An assumption that single main propellant tank is used to feed the smaller reservoirs contained in the MEMS fabricated needle units is made. 


$$
m_{\text {total }}=N_{\frac{\text { thrusters }}{\mathrm{s} / \mathrm{c}}}\left(m_{s}+m_{\text {neutral }}+\frac{m_{p p u}}{2}\right)+m_{\text {feed }}
$$

\section{C.3 FEEP Thruster Design Equations}

The design methodology discussed in Section 5.1.3 is carried out using the following set of equations, which relate the salient variables of the FEEP thruster propulsion system to each other. All quantities are in MKS units; lengths are in meters, masses are in kilograms and times are in seconds resulting in: forces in Newtons, energies in Joules, powers in Watts, frequencies in Hertz, currents in Amperes, voltages in Volts, etcetera.

For the FEEP thruster, the characteristics of operation is set by the voltages. An exponential fit to data presented by Marcuccio et al. [Marcuccio, 1998] for a $5 \mathrm{~mm}$ long slit type FEEP thruster shows that the current behaves as indicated in Equation C.26, where $V_{e}$ and $\mathrm{V}_{\mathrm{a}}$ are the emitter and accelerator voltages, respectively (as illustrated in Figure 4.17) and $\mathrm{N}$ is the length of the slit normalized by $5 \mathrm{~mm}$ (or the number of times longer the slit being designed is than the nominal length). The absolute value of the accelerator voltage is taken because it is normally negative with respect to the spacecraft while the emitter voltage is usually positive.

$$
I_{e}=N\left(2 \times 10^{-7}\right)\left(e^{0.0011\left(V_{e}+\left|V_{a}\right|\right)}\right)
$$

The thrust is derived from the product of the current, the charge to mass ratio of the ions and the exhaust velocity and is also presented by Marcuccio [Marcuccio, 1998]. The additional trigonometric factors are the result of the dispersion of the beam and cause a slight loss of thrust from the ideal case.

$$
\begin{gathered}
F=I_{e} \sqrt{2 \frac{m_{\mathrm{Cs}}}{e} \mathrm{~V}_{e}}\left(\frac{\sin \alpha}{\alpha}\right)\left(\frac{\sin \beta}{\beta}\right) \\
F=N\left(1.666 \times 10^{-3}\right)\left(2 \times 10^{-7}\right) 0.916 \sqrt{\mathrm{V}_{\mathrm{e}}}\left(e^{0.0011\left(\mathrm{~V}_{e}+\left|\mathrm{V}_{a}\right|\right)}\right)
\end{gathered}
$$


The specific impulse is defined by the same equation as for the colloid thruster, but because the charge to mass ratio of a cesium ion is fixed (assuming only singly ionized atoms), it is only a function of the emitter voltage, $\mathrm{V}_{\mathrm{e}}$.

$$
\begin{gathered}
I_{s p}=\frac{1}{g_{o}} \sqrt{2 e \mathrm{~V}_{\mathrm{e}} / m_{\mathrm{Cs}}} \\
I_{s p}=122.3 \sqrt{\mathrm{V}_{\mathrm{c}}}
\end{gathered}
$$

The current collected by the accelerator electrode is made up of contributions from ionized cesium ions impinging upon it, secondary electron sputtering as a result of these impingements and possible glow discharge effects. The expression in Equation C.29 is a rough approximation to data presented in [Marcuccio, 1998]. Generally, the value of 0.2 is used at higher voltage operation and the value of 0.3 is used at lower voltages. This factor determines the efficiency of the thruster.

$$
I_{a}=0.2 \rightarrow 0.3 I_{e}
$$

The total power expenditure is just the sum of the power in the ion beam and the power in the stream of ions that impinges on the accelerator electrode.

$$
P_{i n}=I_{e} \mathrm{~V}_{e}+I_{a} \mathrm{~V}_{a}
$$

The power required to neutralize the ion beam is arrived at in the same way as is done for the colloid thruster (see Equation C.18).

$$
P_{\text {neutral }}=500 I_{e}
$$

The masses for the subsystem components for the FEEP thruster are linear fits to data gathered by Mueller on various FEEP designs that have actually been built, either for laboratory testing or prototyping [Mueller, 2000].

$$
m_{s}=\left(5.384 \times 10^{-4}\right) 10^{6} F_{\text {nominal }}+0.446
$$




$$
m_{p p u}=\left(1.538 \times 10^{-4}\right) 10^{6} F_{n o m i n a l}+0.985
$$

The mass of the neutralizer is estimated from the same source as the neutralizer for the colloid thruster (see Equation C.22). In addition, the two following equations (Equation C.35 and Equation C.36) are the same as those used for the colloid thruster.

$$
\begin{gathered}
m_{\text {neutral }}=3 \times 10^{-2} \\
m_{\text {prop }}=m_{\mathrm{s} / \mathrm{c}}\left(1-e^{-\left(\frac{\Delta \mathrm{v}}{I_{s p} g_{0}}\right)}\right) \\
P_{\text {total }}=P_{\text {neutral }}+P_{\text {in }}
\end{gathered}
$$

The mass sum for a spacecraft with a FEEP thruster system is the same as that for the colloid thruster system except for the absence of a mass associated with a propellant feed system. This is because the FEEP design incorporates the propellant storage area in the body of the thruster unit.

$$
m_{\text {total }}=N_{\frac{\text { thrusters }}{\mathrm{s} / \mathrm{c}}}\left(m_{s}+m_{\text {neutral }}+\frac{m_{\text {ppu }}}{2}\right)+m_{\text {prop }}
$$

\section{C.4 Cold Gas Thruster Design Equations}

The design methodology discussed in Section 5.1.4 is carried out using the following set of equations, which relate the salient variables of the cold gas propulsion system to each other. All quantities are in MKS units; lengths are in meters, masses are in kilograms and times are in seconds resulting in: forces in Newtons, energies in Joules, powers in Watts, frequencies in Hertz, currents in Amperes, voltages in Volts, etcetera.

Equation C.38 says that the thrust required to be produced by the nozzle of the cold gas thruster is the impulse bit divided by the minimum time for which the valve can be opened. This is the way the equation was used in conjunction with the simulation, to 
determine the impulse bit and the thruster size. However, a more physical way to think about this relationship is that the impulse bit is the product of the nozzle's thrust and the minimum amount of time for which the valve can be opened.

$$
F=\frac{I_{b i t}}{w_{p}}
$$

The area of the throat of the nozzle is computed from an approximation to the normal thrust equation, as seen below. The chamber pressure $\mathrm{p} 1$ is determined from data obtained in [Bzibziak, 2000] and [Mueller, 2000]. Mainly this equation is used as a check to make sure that nozzle throat is not smaller than what can be reasonably manufactured.

$$
A_{t}=\frac{F}{C_{F} p_{1}}
$$

As with the colloid and FEEP thruster systems the propellant required for a given mission was simply estimated by using the rocket equation and assuming the approximate specific impulse of cold nitrogen gas thrusters $(\sim 65 \mathrm{~s})$.

$$
m_{\text {prop }}=m_{\mathrm{s} / \mathrm{c}}\left(1-e^{-\left(\frac{\Delta \mathrm{v}}{I_{s p} g_{0}}\right)}\right)
$$

The following three equations were used to make estimates of the size of the propellant storage tank, which often constitutes the majority of the mass for cold gas propulsion systems. The ideal gas equation is used with the propellant mass approximation and an estimate of the tank pressure, based on information in [Larson, 1992], to find the volume the propellant would occupy (Equation C.41). Then, using carbon fiber composite as the tank material and reasonable dimensions (compared to the spacecraft size) for cylindrical tanks, the wall thickness and therefore the tank mass were computed (Equations C.42 and C.43) assuming a factor of safety (FOS) of 1.25 as suggested in [Larson, 1992]. The equation for the feed system hardware mass (Equation C.43) includes the propellant tank mass plus an extra thirty percent as advised in [Larson, 1992]. 


$$
\begin{gathered}
V_{\text {gas }}=\frac{m_{\text {prop }} R_{\text {gas }} T}{P} \\
t_{\text {wall }}=\frac{P_{\text {tank }} r_{\text {tank }} F O S}{2 \sigma_{u l t}} \\
m_{\text {feed }}=1.3\left(2 \pi r_{\text {tank }} L t_{\text {wall }} \rho_{a l}\right)
\end{gathered}
$$

The mass of the associated cold gas thruster nozzle and valve (Equation C.44) were approximated from data from [Bzibziak, 2000] and [Mueller, 2000], as was the power necessary to operate the valves (Equation C.45).

$$
\begin{gathered}
m_{s}=1 \times 10^{-2} \mathrm{~kg} \\
P_{\text {valve }}=10 \rightarrow 15 \mathrm{~W}
\end{gathered}
$$

Similarly to the other propulsion systems, the cold gas thruster system's total mass for a single spacecraft is the sum of the masses of the individual components, assuming there is a central propellant tank.

$$
m_{\text {total }}=m_{\text {prop }}+m_{\text {feed }}+N_{\frac{\text { thrusters }}{\mathrm{s} / \mathrm{c}}} m_{s}
$$


